\title{
WestVirginiaUniversity
}

THE RESEARCH REPOSITORY @ WVU

Graduate Theses, Dissertations, and Problem Reports

2013

\section{Thrombospondin: An emerging keystone in skeletal muscle structure and function}

\author{
Gerald Nelson Audet \\ West Virginia University
}

Follow this and additional works at: https://researchrepository.wvu.edu/etd

\section{Recommended Citation}

Audet, Gerald Nelson, "Thrombospondin: An emerging keystone in skeletal muscle structure and function" (2013). Graduate Theses, Dissertations, and Problem Reports. 3647.

https://researchrepository.wvu.edu/etd/3647

This Dissertation is protected by copyright and/or related rights. It has been brought to you by the The Research Repository @ WVU with permission from the rights-holder(s). You are free to use this Dissertation in any way that is permitted by the copyright and related rights legislation that applies to your use. For other uses you must obtain permission from the rights-holder(s) directly, unless additional rights are indicated by a Creative Commons license in the record and/ or on the work itself. This Dissertation has been accepted for inclusion in WVU Graduate Theses, Dissertations, and Problem Reports collection by an authorized administrator of The Research Repository @ WVU.

For more information, please contact researchrepository@mail.wvu.edu. 


\title{
Thrombospondin: An emerging keystone in skeletal muscle structure and function
}

\section{Gerald Nelson Audet}

\author{
Dissertation submitted to \\ The School of Medicine at West Virginia University \\ in partial fulfillment of the \\ requirements for the degree of: \\ Doctor of Philosophy \\ in \\ Exercise Physiology \\ Dr. I. Mark Olfert \\ Dr. Stephen Alway \\ Dr. Brent A. Baker \\ Dr. John Hollander \\ Dr. Emidio Pistilli
}

Division of Exercise Physiology

Morgantown, West Virginia

2013 


\section{Abstract \\ Thrombospondin: An emerging keystone in skeletal muscle structure and function \\ Gerald N. Audet}

Our objective was to elucidate the effects of TSP-1 on skeletal muscle structure and function, and its potential role in the progression of skeletal muscle dysfunction. The central hypothesis was that TSP-1 is a critical negative regulator of skeletal muscle angiogenesis. In chapter 3, we used a TSP-1 mimetic delivered by mini osmotic pumps to show that TSP-1 can reduce capillarity across three distinct muscle types. We found that those given the mimetic had a 35\% decrease in soleus capillarity, a 20\% decrease in the gastrocnemius, and an $11 \%$ decrease in the plantaris. This confirmed the critical role that TSP-1 plays in physiological skeletal muscle angiogenesis. Despite previous evidence showing that TSP-1 is an important regulator of apoptosis in tumorigenesis, we found no apoptosis in the skeletal muscle of those animals treated with the TSP-1 mimetic, as measured by TUNEL staining or cell death ELISA. Further, again using this same mimetic, in chapters 3 and 4 we failed to find any change in whole body exercise, as measured by maximal running speed, or direct muscle function as measured by $e x$ vivo muscle simulation, in relation to the TSP-1 induced capillary rarefaction. This may further support the notion that it is not simply one factor that controls exercise capacity, but a combination of many different components and systems. Further, previous evidence suggested that a TSP-1/TNF $\alpha$ axis could be controlling skeletal muscle capillarity rarefaction in chronic systemic inflammation. In chapter 5 we examined the role overexpression of TNF $\alpha$ has on skeletal muscle capillary regression in a mouse model of chronic lung disease. Despite showing a 16\% decrease in capillarity of the soleus, we found no change in TSP-1 expression. What 
factors are at play in this specific disease model remains unknown; however our data suggests that TSP-2 could be central to pathological capillary regression seen with this model. Finally, in chapter 4 we also suggest that TSP-1 may be playing a role in the regulation of mitochondrial electron transport enzyme activity, and hence perhaps mitochondrial function. This could provide a new and exciting role for TSP-1. This will require further investigation. Together, the studies in this document build upon data showing that skeletal muscle angiogenesis is a dynamic process controlled by positive and negative angiogenic proteins. It provides new and exciting evidence for the importance of negative angiogenic proteins, specifically that of TSP-1 and TSP2, and lays the groundwork for investigation into potential medical therapies targeting aberrant angiogenesis in skeletal muscle. 
Gerald N. Audet

Doctoral of Exercise Physiology

West Virginia University, School of Medicine

Department of Exercise Physiology

2013

Chair

Dr. I. Mark Olfert

Committee Members

Dr. Stephen Alway

Dr. Brent Baker

Dr. John Hollander

Dr. Emidio Pistilli

Number of Pages: 201 


\section{Table of Contents}

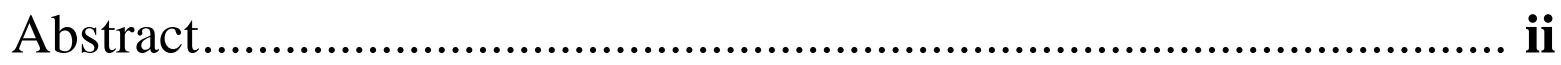

Table of Contents ....................................................................... v

List of Tables ......................................................................... vii

List of Figures............................................................................ viii

Chapter 1: Introduction ............................................................1

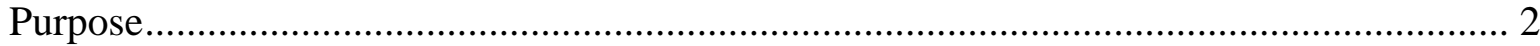

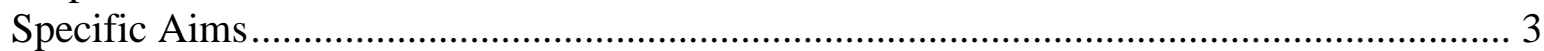

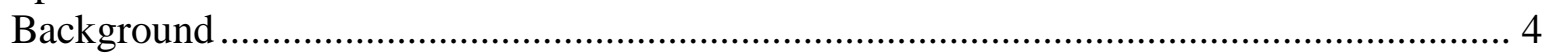

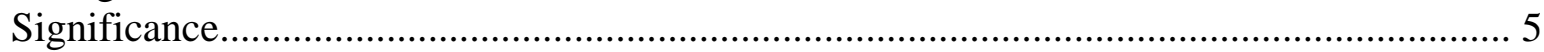

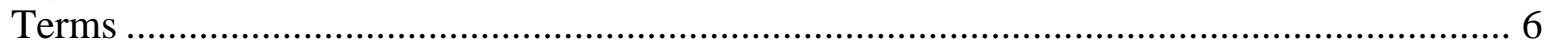

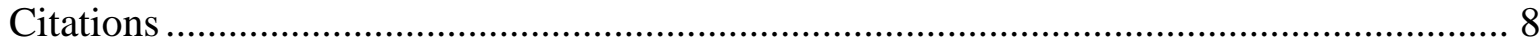

Chapter 2: Literature Review ..................................................... 12

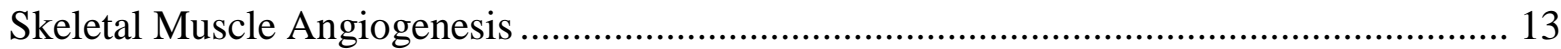

Thrombospondin-1: An important regulator of angiogenesis........................................ 19

Skeletal Muscle Angiogenesis, Chronic Disease, and Chronic Inflammation .................... 27

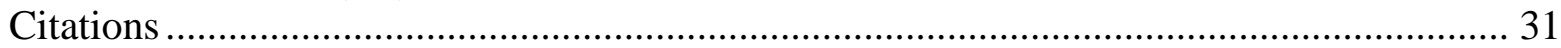

Chapter 3: Chronic delivery of a thrombospondin-1 mimetic decreases skeletal muscle capillarity in mice ................................................... 42

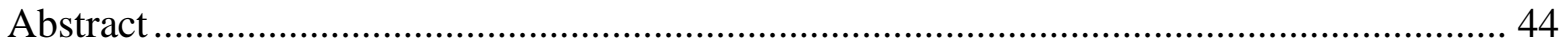

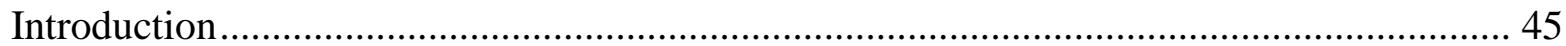

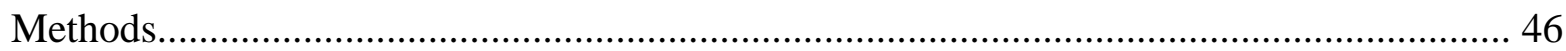

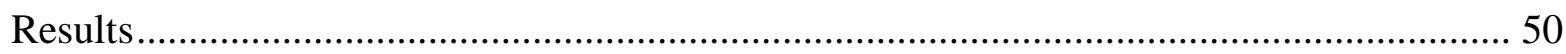

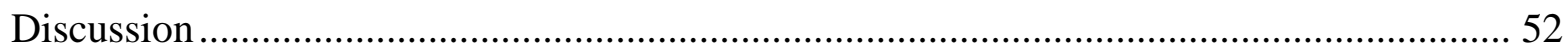

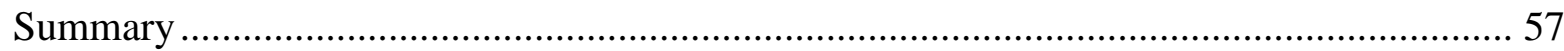

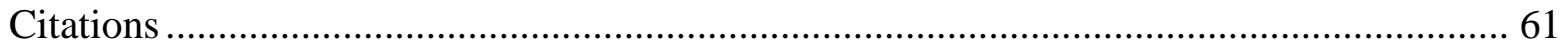




\section{Chapter 4: TSP-1 reduces mitochondrial electron transport chain activity, but does not change isolated skeletal muscle characteristics or in vivo apoptosis ................................................................... 73

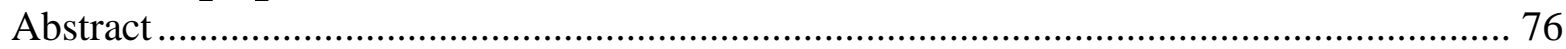

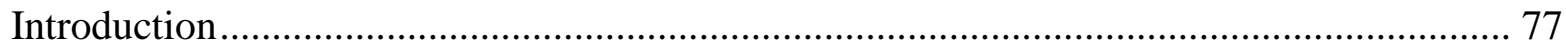

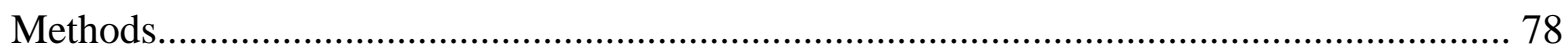

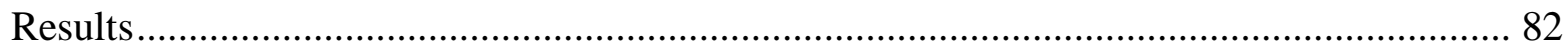

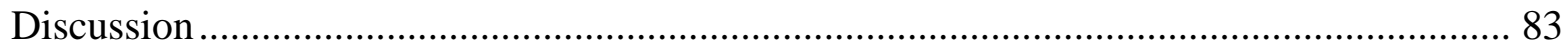

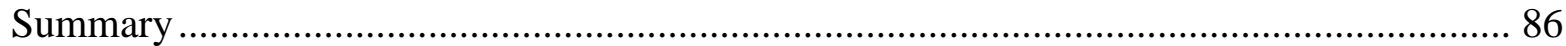

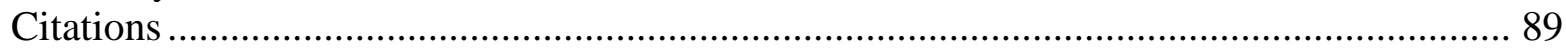

Chapter 5: Systemic inflammation decreases skeletal muscle capillarity but does not change the expression of the important angiogenic protein

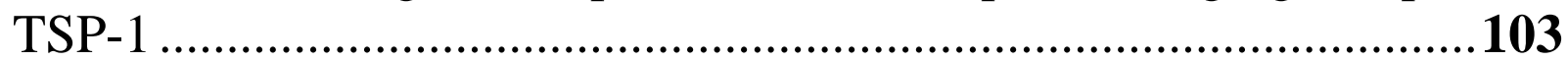

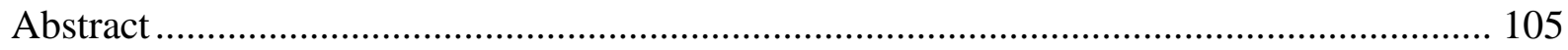

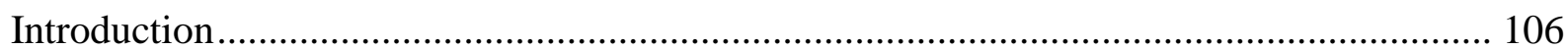

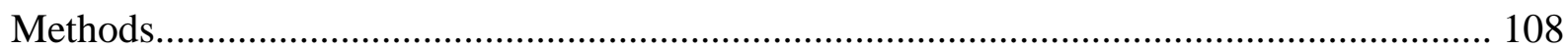

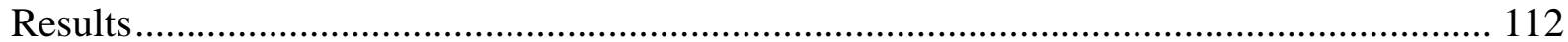

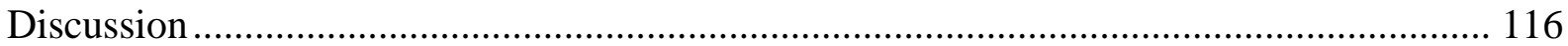

Summary

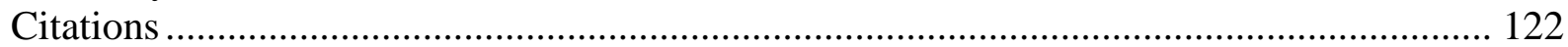

Chapter 6: General Discussion and Future Directions.....................139

Thrombospondin-1 is critical in physiological angiogenesis, but it does not play a role in

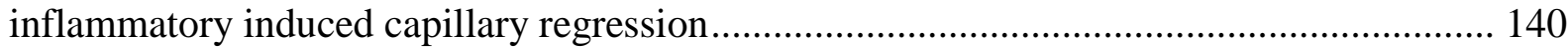

The Thrombospondins play an important role in the angiogenic balance ......................... 143

TSP-1 appears to affect oxidative muscles preferentially ......................................... 147

The role of TSP-1 in skeletal muscle endothelial cell apoptosis remains unclear................ 150

TSP-1 influences mitochondrial enzyme activity .................................................. 152

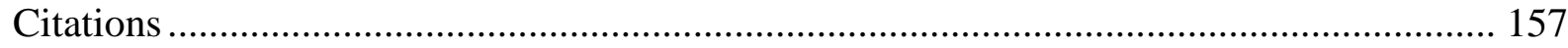

Chapter 7: Future Directions ................................................165

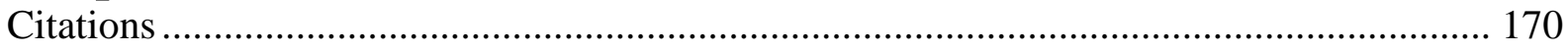


Table of Contents (cont.)

Chapter 8: Appendix ............................................................171

Time course protein expression of TSP-1 following training.......................................... 172

Chronic inflammation does not change skeletal muscle TSP-1 levels in several

different rodent models ...................................................................................... 173

Detraining results in a loss of capillaries, along with increased TSP-1 and VEGF............. 174

"Chronic delivery of a thrombospondin-1 mimetic decreases skeletal muscle

capillarity in mice"

175

"Expression of angiogenic regulators and skeletal muscle capillarity in selectively bred high

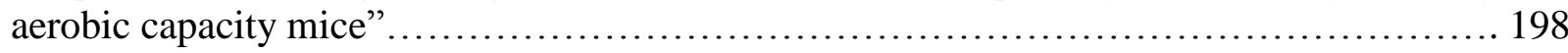




\section{List of Tables}

Chapter 1: Introduction

Chapter 2: Literature Review

Chapter 3: Chronic delivery of a thrombospondin-1 mimetic decreases skeletal muscle capillarity in mice

Table 1: Muscle and Body Masses.

60

Chapter 4: TSP-1 disrupts mitochondrial enzyme activity through the CD36 receptor but does not alter ex vivo skeletal muscle function

Table 1: Age, Body, and Muscle Masses 93

Table 2: Mitochondrial Electron Transport Chain Enzyme Activities ................................. 94

Table 3: Anatomical and physiological characters of the EDL ................................. 95

Table 4: Anatomical and physiological characters of the SOL ............................... 96

Chapter 5: TNF $\alpha$ mediated inflammation decreases skeletal muscle capillarity in association with thrombospondin-2

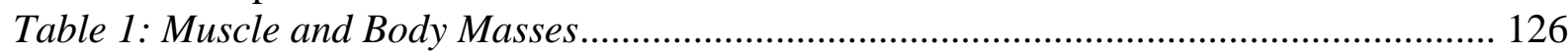

Table 2: TNF $\alpha$ Levels in Serum and Skeletal Muscle ....................................................... 127

Table 3: Mitochondrial Electron Transport Chain Enzyme Activities ................................. 128

Chapter 6: Discussion 


\section{List of Figures}

Chapter 1: Introduction

Chapter 2: Literature Review

Figure 1: The general steps of sprouting and intussception mediated

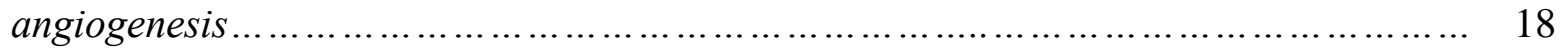

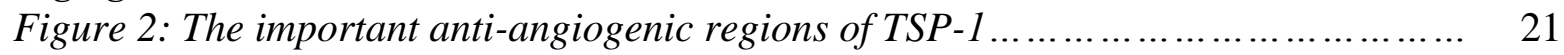

Figure 3: ABT-510 is a mimetic of the type I repeat region of TSP-1 .................. 25

Chapter 3: Chronic delivery of a thrombospondin-1 mimetic decreases skeletal muscle capillarity in mice

Figure 1: Representative figures of the histology sections ............................................. 68

Figure 2: Superficial gastrocnemius capillarity, but not deep, is decreased in the ABT-510

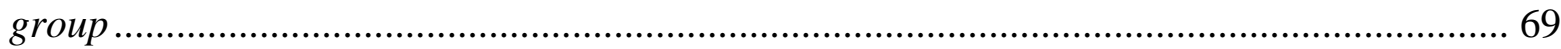

Figure 3: Plantaris and soleus capillarity is decreased in the ABT-510 group .................. 70

Figure 4: Skeletal muscle VEGF protein content is decreased in the ABT-510 group, but

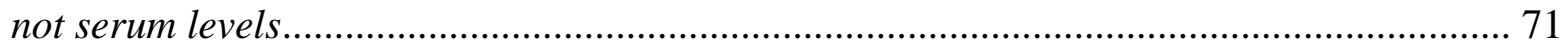

Figure 5: There is no difference in skeletal muscle apoptosis between the mimetic and

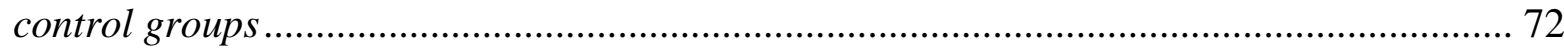

Chapter 4: TSP-1 disrupts mitochondrial enzyme activity through the CD36 receptor but does not alter ex vivo skeletal muscle function

Figure 1: 3 \& 7 days of ABT-510 treatment does not increase cell death ......................... 98

Figure 2: There is a trend for lower peak tetanus force in the SOL of ABT-510 treated

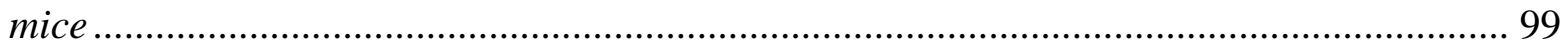

Figure 3: No change in ex vivo muscle fatigue between vehicle and ABT-510 treated

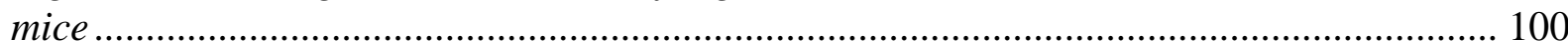

Figure 4: No change in ex vivo muscle fatigue between WT and TSP-1 KO mice ............... 101

Chapter 5: TNF $\alpha$ mediated inflammation decreases skeletal muscle capillarity in association with thrombospondin-2

Figure 1: Endurance running capacity is significantly decreased in $T N F \alpha+$ mice ............. 131

Figure 2: In vivo muscle fatigue is unchanged in $T N F \alpha+$ mice ....................................... 132

Figure 3: Soleus capillarity is decreased in TNFa+ animals, while TSP-1 and VEGF

remain unchanged..................................................................................................... 133

Figure 4: Plantaris capillarity, TSP-1, and VEGF remain unchanged in TNF $\alpha+$ mice...... 134

Figure 5: Angiogenic array reveals of 53 proteins measured, only TSP-2 is changed

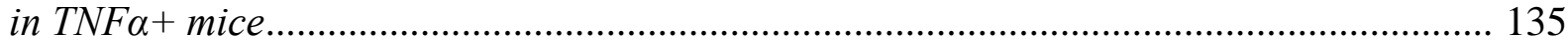

Figure 6: No change in overall ROS content in TNF $\alpha+$ mice .......................................... 136

Figure 7: No change in lipid oxidation in TNF $\alpha+$ mice ................................................. 137

Chapter 6: Discussion 


\section{List of Figures (cont.)}

Chapter 7: Appendix

Figure 1: Time course protein expression of TSP-1 following training

Figure 2: Chronic inflammation does not change skeletal muscle TSP-1 levels in several different rodent models.

Figure 3: Detraining results in a loss of capillaries, along with increased TSP-1 and VEGF 
This page intentionally left blank 


\section{Chapter 1: Purpose, Specific Aims, Introduction, and Significance}




\section{Introduction}

\section{Purpose:}

Meeting the metabolic demands of skeletal muscle during exercise is critical for optimal muscle function. Dysregulation of the delivery of oxygen and nutrients to muscle can contribute to chronic disease morbidity and mortality. Because of this, skeletal muscle capillarity and angiogenesis play essential roles in both health and disease. Angiogenesis is a dynamic process thought to be controlled by the balance between positive and negative angiogenic proteins. While there is an established body of literature pertaining to the positive regulators in angiogenic responses to exercise and disease, the identification and precise role of negative regulators remains poorly understood but may prove to be important in treating the aberrant angiogenesis found in some disease states.

One potentially critical anti-angiogenic protein is thrombospondin-1 (TSP-1). TSP-1 has a wide range of functions, which include the regulation of wound healing, apoptosis, and angiogenesis. As an anti-angiogenic factor, TSP-1 suppresses the essential processes of vascular growth by inhibiting endothelial cell proliferation, survival, and migration. Through these pathways, and others, it is possible that TSP-1 plays a role in skeletal muscle capillary rarefaction and contributes to the skeletal muscle comorbity seen in chronic diseases like cancer, diabetes mellitus, cardiovascular disease (CVD) and chronic obstructive pulmonary disease (COPD).

Our objective is to elucidate the effects of TSP-1 on skeletal muscle structure and function, and its potential role in the progression of skeletal muscle dysfunction. The central hypothesis is that TSP-1 is a critical negative regulator of skeletal muscle angiogenesis. To test our hypothesis, we will examine gain and loss in the function of TSP-1 and explore a potential 
connection between TNF $\alpha$ induced systemic inflammation and TSP-1-induced

pathological/physiological changes in skeletal muscle.

\section{Specific Aims:}

Specific Aim 1: Examine the role of TSP-1 in exercise training-induced skeletal muscle angiogenesis using 1) a TSP-1 mimetic and 2) TSP-1 knock-out mouse model.

In wild-type (WT) mice administered a TSP-1 mimetic, we hypothesize that exercise training induced angiogenesis will be attenuated or ablated. We expect to find greater muscle fatigue, persistently lower levels of positive angiogenic proteins, and decreased exercise and skeletal muscle performance when compared to WT trained animals.

In TSP-1 KO mice, we hypothesize exercise training induced angiogenesis will be increased, and the time course of capillary adaptation will be accelerated compared to WT animals. We also expect to find decreased muscle fatigue, higher levels of positive angiogenic proteins, and increased exercise and skeletal performance when compared to WT trained animals.

Specific Aim 2: Examine the connection between TNFa-and TSP-1 regulated skeletal muscle capillarity and exercise capacity

$\mathrm{TNF} \alpha$ is an inflammatory cytokine that appears central to many chronic diseases, including COPD and CVD. We hypothesize that chronic TNF $\alpha$ overexpression will produce increased chronic systemic inflammation leading to increased skeletal muscle TSP-1 expression, which in turn will decrease muscle capillarity, decrease exercise capacity, and decrease skeletal muscle performance compared to control animals. 


\section{Background:}

Skeletal muscle dysfunction can be a significant barrier in the daily lives of those suffering with chronic disease, such as chronic heart failure (CHF), chronic obstructive pulmonary disease (COPD), diabetes mellitus, and cancer [1-8]. While COPD patients exhibit altered muscle fiber composition, decreased muscle fiber size, decreased skeletal muscle capillarity and decreased overall strength [9-11], the origin of chronic skeletal muscle pathology remains poorly understood.

Skeletal muscle capillarity is an important factor in normal skeletal muscle function. An increase in skeletal muscle capillarity occurs with exercise training and this in turn helps to meet the demand for more oxygen and nutrients. In chronic disease decreased skeletal muscle capillary-to-fiber ratio $(\mathrm{C}: \mathrm{F})$ likely contributes to reductions in overall exercise capacity $[1,12]$. Indeed, there is a significant decrease in $\mathrm{C}: \mathrm{F}$ in $\mathrm{CHF}$ and COPD, as well as evidence of a decreased C:F in diabetes mellitus, the metabolic syndrome, and obesity $[1,12-14]$. While decreased C:F is emerging as an important contributor to disease morbidity, the molecular mediators of this process, and the pathways responsible, are still poorly understood.

Thrombospondin-1 (TSP-1) is a large (450kD) extracellular protein that has a wide array of functions [15]. First discovered for its role in wound healing, it has been shown to play roles in apoptosis, inflammation, nitric oxide signaling, and calcium binding [14, 16-19]. Acting through multiple receptors, including the essential receptors CD36 and CD47, TSP-1 has been shown to prevent endothelial cell adhesion, growth, and migration, and therefore it is implicated as a key negative regulator of angiogenesis and vascular homeostasis. Decreased TSP-1 has been shown to increase tumor vessel growth, and pharmacological administration of several different TSP-1 mimetics has helped decrease tumor size and disease progression in canines and 
mice [20-23]. More recently, TSP-1 mRNA and protein levels have been shown to be important in acute responses to exercise [24], and it's suggested that TSP-1 may play an important role in regulating exercise-induced skeletal muscle angiogenesis [25]. Also, recent evidence shows that hindlimb unloading in rats increases TSP-1 in association with decreases in skeletal muscle C:F ratio [26]. While these data hint at the importance of TSP-1, they are largely circumstantial and there is need for direct evidence showing that TSP-1 plays a role in normal physiological function, as well as in the development of skeletal muscle dysfunction.

It has been suggested that chronic inflammation both contributes to, and results from, the primary pathologies found in CVD, COPD, diabetes mellitus, and obesity [1, 27-29], and therefore likely contributes to skeletal muscle dysfunction [31]. While there are many inflammatory mediators, TNF $\alpha$ has received much attention in COPD and diabetes mellitus [2931], however the mechanisms by which it decreases skeletal muscle function and increases comorbidity is still unknown. There are data to suggest that TNF $\alpha$ may be at the heart of capillary rarefaction seen with chronic pathology. Interestingly, there is a proposed connection between TNF $\alpha$ and TSP-1 that shows TNF $\alpha$ acts to increase human skeletal myoblast expression of TSP-1 [32] in a tissue specific manner. Additionally, increased systemic TNF $\alpha$ has been shown to increase circulating TSP-1 protein levels in humans [33]. There is also evidence that TNF $\alpha$ KO mice have increased running capacity [34], suggesting that TNF $\alpha$, perhaps via lowered basal TSP-1 expression, may play a role in altering muscle capillarity. Collectively these data point to a potential mechanism that may link chronic inflammation, through TNF $\alpha$, in the development of skeletal muscle capillary rarefaction, through TSP-1, resulting in overall muscle dysfunction [35]. 


\section{Significance:}

There is little information about the role that angiogenic inhibitors play in the etiology of skeletal muscle dysfunction. These data will add significantly to the understanding of the role and importance of TSP-1 in regulating skeletal muscle structure and function in basal physiology, exercise, and disease. Indeed, if TSP-1 can be confirmed as a central factor in regulating skeletal muscle angiogenesis and vascular function, it may provide a potential therapeutic target aimed at alleviating skeletal muscle capillary rarefaction and vascular dysfunction found in many chronic diseases.

\section{Terms:}

3TSR: Type I repeat region of Thrombospondin-1

ABT: $30 \mathrm{mg} / \mathrm{kg} / \mathrm{day}$ ABT-510 Treated Wild-type Mice

ABT-510: Abbot Laboratories Compound 510

bFGF: Basic Fibroblast Growth Factor

C:F: Capillary to Fiber Ratio

CD: Capillary Density

CD36 KO: CD36 whole-body KO mice

CD36: Cluster of Differentiation 36

CD47/IAP: Cluster of Differentiation 47

CHF: Chronic Heart Failure

CON: Control mice

COO region: Carboxy terminal region of TSP-1

COPD: Chronic Obstructive Pulmonary Disease

CVD: Cardiovascular Disease

DM: Diabetes Mellitus

EC: Endothelial Cell

EDL: extensor digitorum longus

EGF: Epidermal Growth Factor

eNOS: Endothelial Nitric Oxide Synthase

FCSA: Fiber Cross Sectional Area

Flk-1/VEGFR-2: Vascular Endothelial Growth Factor Receptor 2

GA: Gastrocnemius

IGF: Insulin-like Growth Factor 
IL: Interluekin

IP: Inducible Protein

LRP-1: lipoprotein receptor-related protein-1

MMP: Matrix Metalloproteinase

mRNA: Messenger Ribonucleic acid

NO: Nitric Oxide

PLT: Plantaris

P-VEGFR-2: Phosphorylated Vascular Endothelial Growth Factor

Receptor 2

sGC: Soluble guanylyl cyclase

sGK-1: soluble glucocorticoid-inducible kinase

SOL: Soleus

TGF $\beta$ : Transforming Growth Factor Beta

TNFa: Tumor Necrosis Factor Alpha

TNF $\alpha+$ : Tumor Necrosis Factor Alpha Lung-specific Overexpressing Mice

TSP-1 KO: Full-body Thrombospondin-1 KO

TSP-1: Thrombospondin-1

TSP-2: Thrombospondin-2

VEGF: Vascular Endothelial Growth Factor

WT: Wild-type mice

$\alpha 3 \beta 1$ : Alpha-3 Beta-1 Integrin 
Citations

1. Gosker, H.R., et al., Skeletal muscle dysfunction in chronic obstructive pulmonary disease and chronic heart failure: underlying mechanisms and therapy perspectives. The American Journal of Clinical Nutrition, 2000. 71(5): p. 1033-1047.

2. Gosker, H.R., et al., Striking Similarities in Systemic Factors Contributing to Decreased Exercise Capacity in Patients With Severe Chronic Heart Failure or COPD*. Chest, 2003. 123(5): p. 1416-1424.

3. Tsutsui, H., et al., Oxidative stress in cardiac and skeletal muscle dysfunction associated with diabetes mellitus. Journal of Clinical Biochemistry and Nutrition, 2010. 48(1): p. 6871.

4. $\quad$ Szentesi, P., et al., Depression of force production and ATPase activity in different types of human skeletal muscle fibers from patients with chronic heart failure. Journal of Applied Physiology, 2005. 99(6): p. 2189-2195.

5. Muscaritoli, M., et al., Prevention and treatment of cancer cachexia: New insights into an old problem. European Journal of Cancer, 2006. 42(1): p. 31-41.

6. BERNARD, S., et al., Peripheral Muscle Weakness in Patients with Chronic Obstructive Pulmonary Disease. Am. J. Respir. Crit. Care Med., 1998. 158(2): p. 629-634.

7. Harrington, D., et al., Skeletal Muscle Function and Its Relation to Exercise Tolerance in Chronic Heart Failure. Journal of the American College of Cardiology, 1997. 30(7): p. 1758-1764.

8. Sullivan, M.J. and M.H. Hawthorne, Exercise intolerance in patients with chronic heart failure. Progress in Cardiovascular Diseases. 38(1): p. 1-22.

9. Gosker, H.R., et al., Skeletal muscle fibre-type shifting and metabolic profile in patients with chronic obstructive pulmonary disease. European Respiratory Journal, 2002. 19(4): p. 617-625.

10. Casaburi, R., Skeletal Muscle Function in COPD*. Chest, 2000. 117(5 suppl 1): p. 267S271S.

11. Ho Cheol Kim, M.M., and Saban NA Hussain, Skeletal muscle dysfunction in patients with chronic obstructive disease. International Journal of Chronic Obstructive Pulmonary Disease, 2008. 3(4): p. 637-658.

12. Kivelä, R., et al., Effects of experimental type 1 diabetes and exercise training on angiogenic gene expression and capillarization in skeletal muscle. The FASEB Journal, 2006. 20(9): p. 1570-1572.

13. Frisbee, J.C., Reduced nitric oxide bioavailability contributes to skeletal muscle microvessel rarefaction in the metabolic syndrome. American Journal of Physiology Regulatory, Integrative and Comparative Physiology, 2005. 289(2): p. R307-R316.

14. Varma, V., et al., Thrombospondin-1 Is an Adipokine Associated With Obesity, Adipose Inflammation, and Insulin Resistance. Diabetes, 2008. 57(2): p. 432-439.

15. Jack, L., The functions of thrombospondin-1 and-2. Current Opinion in Cell Biology, 2000. 12(5): p. 634-640.

16. Jimenez, B., et al., Signals leading to apoptosis-dependent inhibition of neovascularization by thrombospondin-1. Nature medicine, 2000. 6(1): p. 41-8.

17. Lange-Asschenfeldt, B., et al., Increased and prolonged inflammation and angiogenesis in delayed-type hypersensitivity reactions elicited in the skin of thrombospondin-2deficient mice. Blood, 2002. 99(2): p. 538-545. 
18. Ridnour, L.A., et al., Nitric oxide regulates angiogenesis through a functional switch involving thrombospondin-1. Proceedings of the National Academy of Sciences of the United States of America, 2005. 102(37): p. 13147-13152.

19. Kvansakul, M., J.C. Adams, and E. Hohenester, Structure of a thrombospondin Cterminal fragment reveals a novel calcium core in the type 3 repeats. EMBO J, 2004. 23(6): p. 1223-1233.

20. Ren, B., et al., Regulation of tumor angiogenesis by thrombospondin-1. Biochimica et Biophysica Acta (BBA) - Reviews on Cancer, 2006. 1765(2): p. 178-188.

21. Hoekstra, R., et al., Phase I Safety, Pharmacokinetic, and Pharmacodynamic Study of the Thrombospondin-1-Mimetic Angiogenesis Inhibitor ABT-510 in Patients With Advanced Cancer. Journal of Clinical Oncology, 2005. 23(22): p. 5188-5197.

22. Reiher, F.K., et al., Inhibition of tumor growth by systemic treatment with thrombospondin-1 peptide mimetics. International Journal of Cancer, 2002. 98(5): p. 682689.

23. Rusk, A., et al., Preclinical Evaluation of Antiangiogenic Thrombospondin-1 Peptide Mimetics, ABT-526 and ABT-510, in Companion Dogs with Naturally Occurring Cancers. Clinical Cancer Research, 2006. 12(24): p. 7444-7455.

24. Olfert, I.M., et al., Temporal thrombospondin-1 mRNA response in skeletal muscle exposed to acute and chronic exercise. Growth Factors, 2006. 24(4): p. 253-259.

25. Olfert, I.M. and O. Birot, Importance of Anti-angiogenic Factors in the Regulation of Skeletal Muscle Angiogenesis. Microcirculation, 2011. 18(4): p. 316-330.

26. Roudier, E., et al., Angio-adaptation in unloaded skeletal muscle: new insights into an early and muscle type-specific dynamic process. The Journal of Physiology, 2010. 588(22): p. 4579-4591.

27. Danesh, J., et al., Low grade inflammation and coronary heart disease: prospective study and updated meta-analyses. BMJ, 2000. 321(7255): p. 199-204.

28. $\mathrm{Xu}, \mathrm{H}$., et al., Chronic inflammation in fat plays a crucial role in the development of obesity-related insulin resistance. The Journal of Clinical Investigation, 2003. 112(12): p. 1821-1830.

29. Duncan, B.B., et al., Low-Grade Systemic Inflammation and the Development of Type 2 Diabetes. Diabetes, 2003. 52(7): p. 1799-1805.

30. Gan, W.Q., et al., Association between chronic obstructive pulmonary disease and systemic inflammation: a systematic review and a meta-analysis. Thorax, 2004. 59(7): p. 574-580.

31. Wellen, K.E. and G.S. Hotamisligil, Inflammation, stress, and diabetes. The Journal of Clinical Investigation, 2005. 115(5): p. 1111-1119.

32. Salajegheh, M., et al., Upregulation of thrombospondin-1(TSP-1) and its binding partners, CD36 and CD47, in sporadic inclusion body myositis. Journal of Neuroimmunology, 2007. 187(1-2): p. 166-174.

33. Lutz, J., et al., Increased Plasma Thrombospondin-1 (TSP-1) Levels Are Associated with the TNF $\alpha$-308A Allele in Children with Juvenile Dermatomyositis. Clinical Immunology, 2002. 103(3): p. 260-263.

34. Netea, M.G., et al., Increased voluntary exercise in mice deficient for tumour necrosis factor- $\alpha$ and lymphotoxin- $\alpha$. European Journal of Clinical Investigation, 2007. 37(9): p. 737-741. 
35. Frazier, E.P., et al., Age-dependent regulation of skeletal muscle mitochondria by the thrombospondin-1 receptor CD47. Matrix Biology, 2011. 30(2): p. 154-161. 
This page intentionally left blank 


\section{Chapter 2: Literature Review}




\section{$\underline{\text { Skeletal muscle angiogenesis }}$}

\section{Skeletal Muscle: A remarkably adaptable organ}

Skeletal muscle makes up approximately 30 to $40 \%$ of the total mass of the average healthy adult body, dependent on gender. It is an incredibly plastic organ, allowing it to be both energetically economical while also providing the necessary adaptation required to respond to a wide variety of conditions and stimuli. That is, by being extremely adaptable, skeletal muscle provides maximum performance capacity at the minimum energy cost to the rest of the organism. The different systems that make up a muscles overall function (e.g. force production, endurance capacity, etc) respond differently along a spectrum depending on the type and duration of the stimulus, as well as the genetic and environmental differences between individuals. This leads to an vast amount of adaptation that can occur across the many different systems of skeletal muscle, which can include the actual contraction apparatus (i.e. myosin and actin) as well as all the other associated systems (e.g. neuronal input, calcium handling, mitochondrial content, vascularity, etc). The variations in muscle contractile and metabolic systems, and the ability to adapt, gives different muscles throughout the body different functional capacities and provides the organism with a range of kinetic abilities. These range from highforce anaerobic muscles, to low-force high aerobic muscles, and an almost infinite continuum between these two extremes. The changes that occur in skeletal muscle in response to physiological and pathological stimuli have been well reviewed [1-7] and involve a host of molecular mediators.

One of the changes that can occur in skeletal muscle in response to a physiological or pathological stimulus (e.g. exercise, chronic inflammation) is angiogenesis, or capillary growth. Capillaries are the smallest blood vessels in the body and are responsible for the diffusion of 
oxygen and nutrients to the muscle, as well as removal of carbon dioxide and metabolic waste. This is a vital process in skeletal muscle, as muscle is capable of an extremely high metabolic rate that requires both high levels of oxygen and nutrients, as well as the constant removal of various forms of waste. To this end, it has been shown repeatedly in both rodents and humans that with a chronic exercise stimulus there is an increase in the number of capillaries in skeletal muscle. This increase has been shown in terms of overall vessel density and in direct comparison to the number of myocytes, also known as the capillary-to-fiber ratio $(\mathrm{C}: \mathrm{F})[8-20]$. It has been suggested that skeletal muscle capillarity may be one of the most important adaptations to occur with an exercise stimulus [21-24]. Indeed, genetic differences in angiogenic factors and angiogenic expression have been linked to overall exercise capacity, illustrating this important connection $[9,25]$.

\section{Skeletal Muscle Angiogenesis}

The vascular bed in-of-itself is capable of dramatic growth and regression in concert with the other physiological systems of skeletal muscle. Exercise training studies in humans, rodents, and other animal models have shown repeatedly that there is a dramatic increase in capillarity with exercise training in healthy subjects $[7,26,27]$, while detraining and chronic disease decreases capillarity $[14,28]$. There are relatively few studies in skeletal muscle examining what controls angiogenesis, especially when compared to tumorigenesis and other systems in vitro. Further still, what proteins and molecular mediators trigger the growth and regression of the vascular bed in skeletal muscle is still relatively unknown. However, from what data does exist it appears the majority of the angiogenic process in skeletal muscle, and the mediators of this process, are very similar to that of other angiogenic systems, like tumorigenesis [26, 29]. However, unlike in tumorigenesis where hypoxia appears to be the primary trigger, there are a 
striking number of different stimuli that can trigger angiogenesis in vivo in skeletal muscle. While hypoxia has been implicated as one of the largest factors in triggering physiological and pathological angiogenesis in skeletal muscle, $\mathrm{pH}$ level, physical stretch of the muscle, nerve innervation, vibration, and sheer stress have all been implicated in triggering angiogenesis [26, $27,30-38]$. It is likely a combination of all of these factors, and potentially others, which trigger capillary growth in skeletal muscle. The importance of each may vary depending on an additional multitude of factors, including genetics and environment $[9,12]$.

The process of angiogenesis can be broken down into a series of general steps, which will be summarized very briefly here (figure 1). These steps have been intensely studied in tumorigenesis as a way to prevent tumor growth and metastasis. The process is first triggered by a stimulus (e.g. hypoxia, sheer stress) that prompts angiogenic proteins to be released from the tissue being subjected to the stimulus. Many different tissues and cell types have been shown to release angiogenic proteins, including endothelial cells (EC), adipocytes, myocytes, and many others $[9,17,35,39-47]$. An example of one of these angiogenic proteins that is released from a variety of tissues is vascular endothelial growth factor (VEGF) [48-55]. However, there are many other angiogenic factors that can play important roles in angiogenesis [38, 56-58].

Once released angiogenic proteins bind a host of specific and non-specific receptors on EC's and cause the cells to become "activated". This can result in two distinct types of vessel growth. In development and tumorigenesis, as well as in a more limited capacity in adult tissue, new vessels can be formed from the existing networks via vessel sprouting. However, in skeletal muscle and other adult tissues, it is thought that intussusception, or the splitting of intact vessels, may be the primary form of angiogenesis $[59,60]$. Interestingly, depending on the stimulus, 
timing, and amount released, different angiogenic proteins can trigger either or both types of angiogenesis $[26,60]$.

The process of sprouting starts when the EC's push into the surrounding tissue [35, 61]. This requires a complex concert of molecular mediators to break down the basement membrane that holds the vessel and surrounding tissue together, while still retaining vessel integrity as the ECs sprout a new vessel bud. The EC's then migrate along a chemotaxic pathway towards the area that is in need of increased vascularity [26, 62-64]. Matrix metalloproteinases (MMPs), another group of important angiogenic proteins, play a critical role in breaking down the basement membrane and allowing the endothelial cells to migrate forward [58, 65-68].

As the ECs migrate, the extracellular matrix around the vessel is remodeled and stabilized to facilitate the new vessel. Once the budding vessel has reached the area in need, such as a tumor or hypoxic region of skeletal muscle, the endothelial cells narrow and flatten, creating a tube where by plasma and red blood cells can travel to the tissues in need. Finally, the new vessel is stabilized and continues to grow as it loops back to the existing vasculature to create a new capillary-venule loop [26, 62, 64, 69].

Intussusception is slightly different than sprouting in that it does not necessarily require EC migration. Instead, it is the process by which a vessel splits to form two new intact vessels. This is triggered by many of the same stimuli and angiogenic mediators as sprouting [59, 70-72]. Intussceptive angiogenesis starts when a pillar forms in the existing vessel from the endothelial cells on each side of the vessel. These pillars eventually reach each other in the middle of the vessel, and interendothelial cell contact is made. This is stabilized by other cells surrounding the vessel, like pericytes and myofibroblasts. Collagen is then deposited and the post expands and leads to a new hole occurring through the vessel that is sealed from the surrounding tissue. This 
continues to enlarge, splitting the vessel, and the vessel diverges into two new branches. If this continues many times, a single vessel branch can become a mesh-work of new smaller vessels covering much more area, effectively and dramatically increasing overall vascular density. Intussception has been suggested to be the primary form of angiogenesis that occurs in adult tissue $[60,70]$. However, both of these processes seem to be occurring in skeletal muscle, yet what stimulus triggers which, and why, is yet unknown.

\section{Physiological Skeletal Muscle Angiogenesis}

Skeletal muscle capillarity has been shown to be a critical component of exercise training. Dr. Peter Wagner and his colleagues have shown repeatedly that oxygen diffusion to the myocyte is a critical determinant of overall exercise capacity, and that capillarity plays a imperative role in this process $[13,21,26]$. Further, in transgenic mouse models, it has been shown that skeletal muscle angiogenesis is directly linked to whole body exercise capacity [18, $22,23,73]$. In these studies, animals with more capillaries have increased exercise capacity, and the opposite is true for those with decreased capillarity. In humans, skeletal muscle angiogenesis occurs with only a week of aerobic exercise, and in rodents it may occur even more quickly [8, 20]. This adaptation occurs in concert with an increase in expression of positive angiogenic factors, and a decrease in anti-angiogenic factors $[40,74]$. This differential expression has been suggested to serve as a complicated balance and interplay that controls vessel growth. This idea of an angiogenic balance was first introduced by Dr. Judith Folkman almost 30 years ago in tumorigenesis [75], but has since been shown to be applicable to a wide range of physiological and pathological states, including skeletal muscle $[18,23,26]$. Much work has been done in identifying the positive angiogenic factors and their roles in angiogenesis, including potent progrowth proteins like fibroblast growth factor (bFGF), insulin like growth factor (IGF), and 


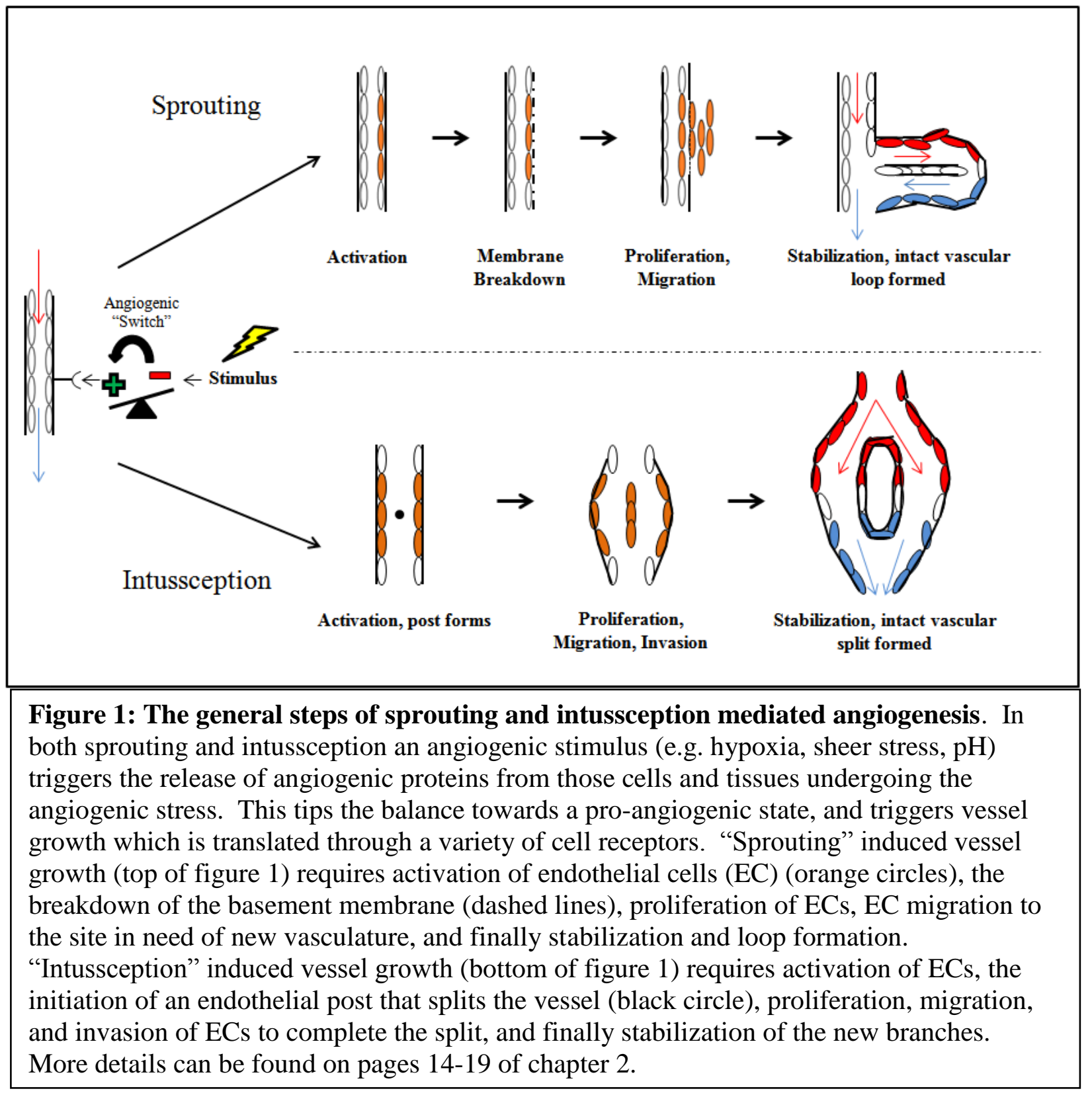

vascular endothelial growth factor (VEGF). Of the long list of positive angiogenic factors discovered, VEGF has been proven to be one of the most ubiquitous and most potent proangiogenic proteins $[9,48,49,51-55,76]$. It has been shown to be essential in many different vascular systems, including skeletal muscle [18, 23].

For some time it was assumed that VEGF and the positive angiogenic proteins were the most important factors in controlling vessel growth in all systems, including in skeletal muscle. 
More recently, anti-angiogenic proteins have been identified as being just as important, or more, than the positive angiogenic proteins [57]. Of these, Thrombospondin-1, or TSP-1, has been shown to be one of the most critical. TSP-1 has been shown to be vital in controlling tumor angiogenesis [77-81]. The theory of this control, the idea of an angiogenic balance, is best explained through the analogy of an automobiles gas and brake pedals. Positive angiogenic factors can be thought of as the "gas-pedal" in angiogenesis, the negative angiogenic factors serve as the "brake-pedal". It has been suggested that without removal of the "brake", even if the "gas-pedal" is full "on", angiogenesis cannot occur. That is, even if pro-angiogenic proteins are elevated, if anti-angiogenic proteins are also elevated, angiogenesis will not occur, or occur to a diminished extent [57]. This lends a potentially essential role to TSP-1 and other antiangiogenic proteins as the primary regulators of angiogenesis.

This is shown in skeletal muscle by looking at mRNA responses to exercise training in the gastrocnemius muscle of rats by two papers $[40,82]$. Together these papers show that VEGF mRNA increases initially with an exercise stimulus- but so does the mRNA of the important angiogenic regulator thrombospondin-1 (TSP-1). Over a period of 3 days of training, the TSP-1 mRNA response is reduced, while the VEGF response stays elevated. This change in mRNA expression occurs along the same timeline as the adaptation to exercise, as capillary adaptation has been shown to occur in rodents between 3 and 7 days [20]. This has led us and others to suggest that TSP-1 may serve as the primary negative angiogenic protein, or "brake", in skeletal muscle angiogenesis.

\section{Thrombospondin-1: An important regulator of angiogenesis}

There has been a wide array of angiogenic proteins indentified in vivo and in vitro. Many of these proteins are responsible for spurring vessel growth and formation. As discussed 
previously, the list of negative angiogenic proteins is relatively smaller, and less well studied, especially in the context of skeletal muscle [29]. However, it is now hypothesized that angiogenesis is controlled as much by negative, as positive angiogenic, proteins. Of the growing list of negative angiogenic proteins, thrombospondin-1 has been shown to be especially potent in several different physiological and pathological conditions.

\section{TSP-1: A multi-domain, multi-function protein}

TSP-1 is a large, $450 \mathrm{kD}$, homo-trimeric extracellular glyco-protein that has been shown to have a wide array of functions $[80,83-85]$. TSP-1 belongs to a family which includes six different proteins (Thrombospondin-1 through Thrombospondin-6). TSP-1 was the first discovered, now over 40 years ago, for its role in wound healing. In this setting, it makes up the majority of the protein content of stimulated $\alpha$-granules in platelets [86]. Upon activation, TSP-1 is released from the $\alpha$-granules where it helps in platelet aggregation and stabilization. It wasn't until some years later, in 1990, when Good et. al. first showed that an unknown potent inhibitor of tumor vascularity was identical in structure to that of TSP-1, and indeed was TSP-1 [79]. They further went on to show in vivo that TSP-1 was a potent anti-angiogenic protein, which could antagonize the actions of other pro-angiogenic proteins under physiological conditions. They suggested that loss of TSP-1 could be a mechanism by which tumors were able to acquire and control vascular growth, allowing unbridled expansion [79].

TSP-1 has multiple functional domains that gives it the ability to interact with a variety of receptors and proteins resulting in a large range of actions throughout many different systems $[78,80,83,85]$. TSP-1 has been shown to have 6 distinct regions, with 4 of these being shown to have significant anti-angiogenic action. This is what is hypothesized to give TSP-1 a greater angiogenic capacity over the closely related TSP-2 protein (and the other members of the TSP 
family), which lacks several of the regions essential to TSP-1's antiangiogenic capacity [80, 85,

87]. Of the four regions found to be important in TSP-1's anti-angiogenic capacity, three are the most significant and most well studied. These are the amino-terminal region, the type I repeats, and carboxy-terminal region (figure 2) $[85,88]$. This review will focus on these regions, however for additional information please see selected sources [78, 80, 83, 85].

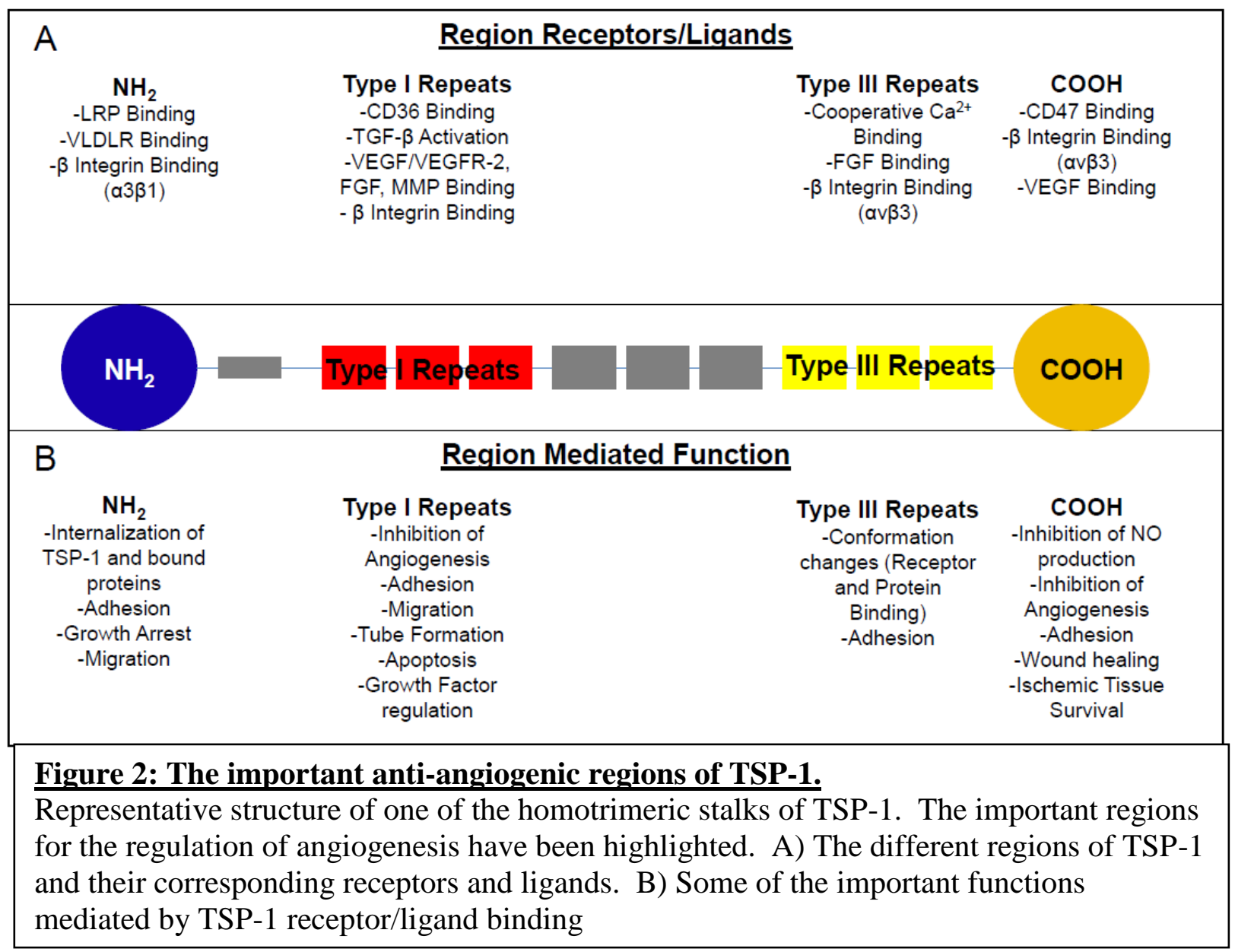

The first of the important anti-angiogenic regions of TSP-1 is the amino terminal end. The amino terminal end has been shown to play a role in attachment, migration, and the endocytosis of TSP-1 in endothelial cells $[78,80,85]$. This region interacts with the $\alpha 3 \beta 1$ integrin, which is potentially the most important of several integrins that TSP-1 can bind and interact with. Integrins in of themselves regulate a wide range of functions, from cell 
attachment, to growth and migration, and have been shown to be important in angiogenesis [8991]. The association between TSP-1 and $\alpha 3 \beta 1$ has been revealed to be significant in some cancers, where it has been suggested that the interaction may play a role in tumorigenic potential $[87,92,93]$. The association of the amino terminal region with $\alpha 3 \beta 1$ has also been shown to function in the innate inflammatory response in damaged tissue. Chronic inflammation has been implicated as a potential mediator of the loss in capillarity seen in some diseases, and may have a component in which TSP-1 plays a pivotal role (discussed in chapter 5, starting on page 96).

The amino terminal end of TSP-1 can also interact with the low-density lipoprotein receptor-related protein-1 (LRP-1). This receptor has been shown to be important in internalization of TSP-1 and catabolization of the protein $[94,95]$. Endocytosis can play an important role in the actions of angiogenic proteins, as has been shown in VEGF [54]. Greenaway and colleagues 2007 showed that binding of VEGF by TSP-1directly, and then internalization of the complex by TSP-1's binding to LRP-1, could effectively reduce many of the markers of angiogenesis- including reducing endothelial cell growth, and an increase in apoptosis [94]. This may be an important mechanism by which TSP-1 indirectly controls angiogenesis, in concert with its direct effects.

TSP-1 also contains an important region known as the type I repeat region (type I), or thrombospondin repeat region (TSR). The type I region are an important portion of the stalk of the protein, where they are repeated three times in succession. This region has been the focus of much intensive study for its potent ability to control angiogenesis and counter other positive angiogenic proteins [84]. This region is only found in TSP-1 and TSP-2, while the other members of the family, TSP-3-6, are missing this important region. The type I region has been shown to work in concert with the amino terminal region to bind the afore mentioned $\alpha 3 \mathrm{~B} 1$ 
integrins [96]. It has also been shown to be the region that can bind and activate the latent antiangiogenic protein transforming growth factor beta (TGF- $\beta$ ) [80, 97-99]. Activation of TGF$\beta$ requires the cleavage of a pro-domain from the full length peptide, resulting in a functional protein. The activation of the TGF- $\beta$ by TSP- 1 requires two specific part of the type I regions, the first to bind TGF- $\beta$ (the WSHWSPW region) and the second to activate it (the RFK region) $[80,85]$. While there is some conflicting data, TGF- $\beta$ has been shown repeatedly to be important in regulating endothelial cell growth and inhibiting angiogenesis [98-105]. It has been suggested that a portion of TSP-1's anti-angiogenic activity is through its activation of latent TGF- $\beta$. While this could certainly play some role in its ability to inhibit endothelial cell growth, TSP-1 also has its own inherent anti-angiogenic capacity, independent of TGF- $\beta$.

One of the most well studied of these inherent anti-angiogenic activities, and one of the important roles for the type I repeats, is the binding of the CD36 receptor [42, 84, 85, 106-112]. This interaction, first identified by Dawson et. al. in 1997, has since been shown to be vitally important in tumorigenesis $[42,85,107,110,112]$. CD36 is a ubiquitously expressed receptor with an array of functions. Of these, it's initial role was thought to be as a simple fatty acid translocation and cholesterol uptake channel, as well as serving as a general multi-ligand scavenger receptor $[107,113]$. Since these discoveries, it has also been shown repeatedly that TSP-1 binds CD36 through the $2^{\text {nd }}$ type I repeat region, an area with a conserved CSVTCG amino sequence $[84,85]$. This interaction has been shown to decrease endothelial cell growth and migration [42]. Additionally, some cancers have been shown to decrease TSP-1 expression, and CD36 expression has also been associated with colon cancer prognosis [111]. Jimenez et. al. 2000 showed that the inhibition of angiogenesis via TSP-1 is deficient in CD36 KO mice [109], suggesting a critical role for this interaction. The same study also showed that TSP-1's 
interaction with CD36 is an important pathway in the induction of endothelial cell apoptosis. Since this important paper was published, several other studies have provided more evidence that TSP-1 is a potent stimulator of apoptosis, and that this could be occurring through the CD36 receptor [109, 114-117]. This induction of apoptosis is likely an important pathway in the antiangiogenic capacity of TSP-1.

TSP-1 has also been shown to bind VEGF directly, and this seems to also be occurring via the type I repeat region. This binding results in the disruption of VEGF induced cell growth and migration, and can ablate the anti-apoptotic actions of VEGF [84, 118, 119]. Further, the type I region of TSP-1, in concert with CD36, has been shown to directly bind the VEGF receptor Flk-1, also known as VEGFR-2, and both decrease VEGF binding as well as inhibit receptor phosphorylation $[110,120,121]$. This interaction may also require the additional TSP-1 receptor CD47 [122]. VEGFR-2 receptor phosphorylation is critical for the downstream effects of the receptor-ligand binding, and provides another mechanism by which TSP-1 is preventing vascular growth and potentially inducing regression [76, 123].

Since the interaction between TSP-1 and CD36 has been identified as an important part of TSP-1's antiangiogenic capacity, several type I mimetics specifically targeting CD36 have been developed to help combat tumorigenesis in human conditions. One example of these is the TSP-1 mimetic ABT-510, developed by Abbot Pharmaceuticals (Figure 3). ABT-510 is a mimetic of the $2^{\text {nd }}$ type I repeat of the TSP-1 stalk (figure 3). There have been several novel substitutions in the amino structure of ABT-510 that result in nanomolar concentrations having an approximately 1000-fold increase in anti-angiogenic activity compared to endogenous TSP-1 in vitro [124]. ABT-510 has been shown to decrease cell growth, decrease cell adhesion, counter the actions of VEGF, and induce apoptosis in vitro, as well as decrease tumor size in 
vivo $[108,116,121,125-130]$. Several other peptides have also been created, and all have shown varying degrees of success, with and without other forms of cancer treatment [130, 131]. These mimetics are in different stages of clinical trial, and pharmaceutical companies continue to try and develop and improve new mimetics $[127,129]$.

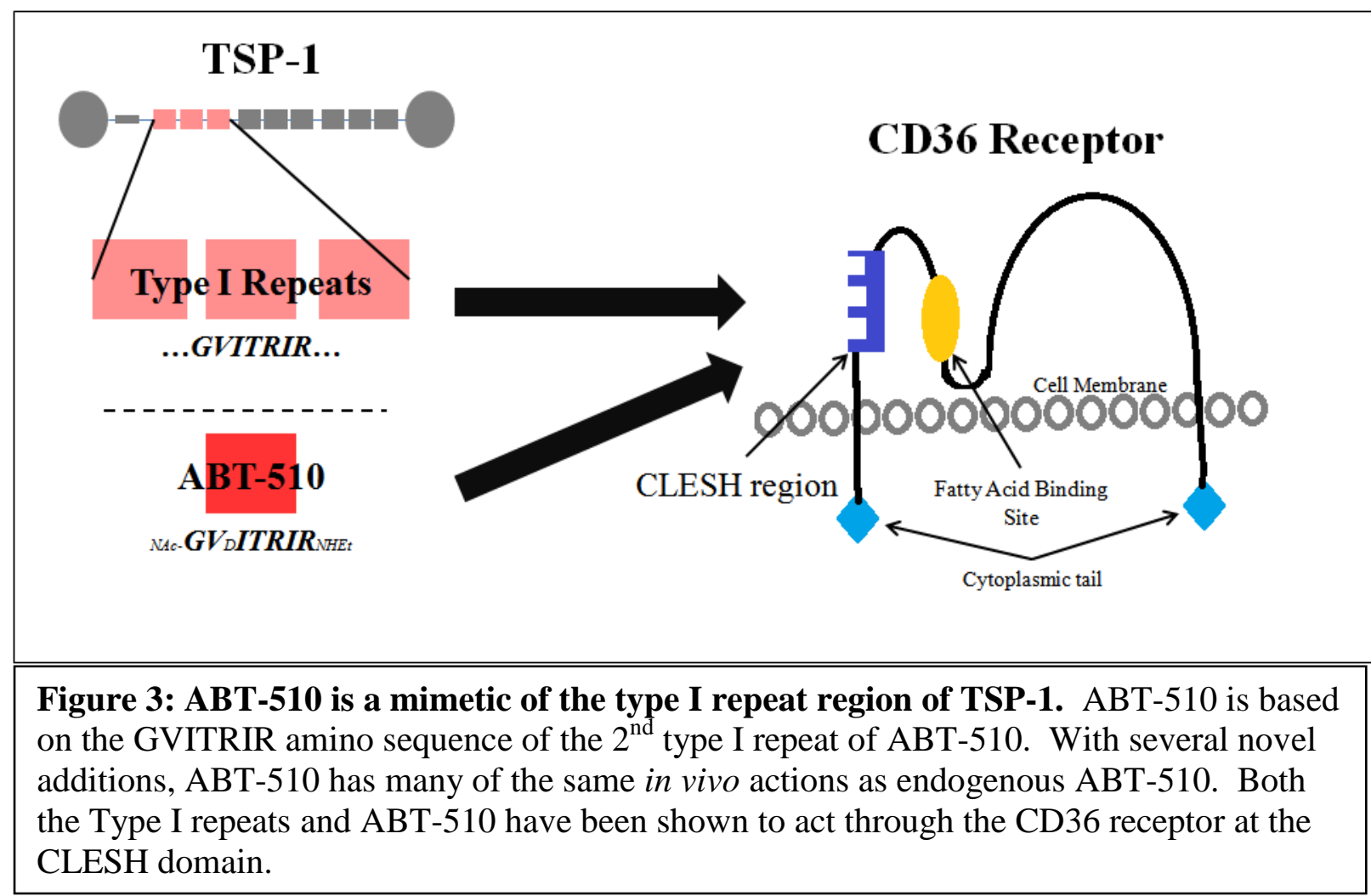

The next portion of TSP-1 that has been shown to play a critical role in its anti-

angiogenic activity is the carboxy-terminal region ( $\mathrm{COO}$ region). Investigation of this region has identified the important TSP-1 receptor CD47, or integrin associated protein (IAP). CD47, like CD36, is a multi-functional protein with a variety of functions, including neutrophil activation, cell adhesion, and cell communication $[132,133]$. CD47 has also been shown to be important in immune system activation, as shown by the susceptibility of the CD47 KO mouse to lethal infections [134]. Also like CD36, the TSP-1/ CD47 interaction has been shown to be powerfully anti-angiogenic. The TSP-1/CD47 axis has been shown to be pro-apoptotic, anti-proliferative, 
and can interact with the VEGFR2 receptor to block angiogenesis, independent of CD36 [80, $122,132,133,135-137]$. While there have been several different down-stream mechanisms suggested for TSP-1/CD47's anti-angiogenic activities, the work by Dr. Jeff Isenberg and colleagues over the last decade have shown that it is likely due to TSP-1s inhibition of nitric oxide (NO) [122, 132, 136, 138-140]. Nitric oxide is a small highly reactive molecule that is critical in signal transduction in many different physiological processes [141]. NO has been shown to be pro-angiogenic and induces endothelial cell growth [139, 142, 143]. TSP-1, acting through CD47, has been shown to block the actions of NO through multiple different mechanisms, including the inhibition of soluble guanylyl cyclase (sGC), cyclic guanosine monophosphate, and cGMP-dependent kinase type I (sGK-1), which are the proteins that translate NO into downstream signals within the cell [138-140]. TSP-1/CD47 has also shown to alter endothelial intracellular calcium release, which is also important in NO signaling [144]. Finally, TSP-1/CD47, as mentioned previously, can block VEGFR-2's downstream actions, which include stimulating NO release via endothelial nitric oxide synthase (eNOS) [122]. Isenberg et al. 2012 suggests that through blocking NO signaling, TSP-1/CD47 could serve as a global regulator of cardiovascular function, which includes it's ant-angiogenic effect [140].

\section{Thrombospondin-1 in Skeletal Muscle}

Through these multitude of effects discussed, TSP-1 has been suggested to be critical in cancer, tumorigenesis, and in endothelial cells. Hundreds of studies spanning many different types of cancer suggest it is critical in the regulation of tumor growth. However, the evidence for TSP-1's actions in skeletal muscle capillarity is significantly less. There is some work to suggest that TSP-1 expression increases with muscle damage, which would be in congruence with its role in wound healing [145]. Also in skeletal muscle, as discussed previously, TSP-1 
mRNA has been shown to increase initially with an exercise stimulus. This has been suggested to be an evolutionarily evolved trait that provides an initial "brake" against un-needed vascular adaptation. However, with exercise training, this mRNA response disappears, and as it does, is associated with vascular adaptation (capillary growth) [40]. Therefore it was suggested that TSP-1 could act as a master controller of angiogenesis in skeletal muscle under physiological conditions.

Perhaps the strongest evidence for the importance of TSP-1 in skeletal muscle comes from [22]. In this study, TSP-1 whole-body knock-outs (TSP-1 KO), were shown to have dramatically increased skeletal muscle capillarity across three distinct muscle types, as well in cardiac tissue [22]. These animals also had significantly increased aerobic exercise capacity, as well as increased levels of VEGF.

Further evidence has shown that in a diabetic insult TSP-1 is increased in skeletal muscle, and skeletal muscle capillarity is decreased [146, 147]. This is also true of hind-limb unloading, where TSP-1 expression increases as capillarity decreases [14]. Interestingly, we have also shown previously that TSP-1 is lower in the plantaris of mice with high-endurance capacity and increased skeletal muscle capillarity [9]. Finally, CD47 KO and TSP-1 KO mice have been shown to have decreased mitochondrial content, which may be another important component of TSP-1's action on skeletal muscle [148]. Given this relatively small body of work, much still needs to be done to determine TSP-1's role and importance in controlling skeletal muscle capillarity under both pathological and physiological conditions.

\section{Skeletal Muscle Angiogenesis, Chronic Disease, and Chronic Inflammation}

Chronic diseases, such as CVD, CHF, COPD, DM, and obesity, are responsible for the largest percentage of deaths in the United States of America (USA) [149]. These diseases are 
costing the USA billions of dollars a year [150-154]. While each of these disease affects the bodies systems in different ways, common between all of them is a chronic systemic level of inflammation that is a result of the primary pathology. This increased chronic systemic inflammation in-of-itself can be as significant to a patients mortality and morbidity, and in some pathologies is implicated as being as important a risk of death as the primary pathology [155$165]$.

An additional important co-morbidity in chronic disease, skeletal muscle dysfunction has been shown in many of the same diseases as chronic systemic inflammation. Indeed, skeletal muscle dysfunction, as measured by decreased overall muscle force, has been associated with decreased life span in congestive heart patients, independent of the primary disease state [166] and it has been shown to be a particularly important comorbidity of COPD [10, 28, 167-173]. Decreased skeletal muscle function has also been shown to be important in a multitude of other diseases [165, 166, 174-180], as well as in aging, bed rest, and obesity [181-184]. As discussed previously, skeletal muscle capillarity is an important component of overall skeletal muscle function, especially endurance capacity. Skeletal muscle capillarity and angiogenesis has been shown to be decreased in these same diseases with global skeletal muscle dysfunction. Indeed, in COPD there has been work to try and identify if a decrease in vascularity is occurring and how it contributes to overall disease morbidity $[10,28,167,169,171,185]$, however to this point it has been mostly correlative in nature.

It has been suggested that chronic systemic inflammation may be the connection between chronic disease and decreased skeletal muscle function and capillarity. This is due in large part to the observation that many of the same diseases that have increased systemic inflammation are associated with decreased skeletal muscle capillarity $[155,158,162,183,186]$. Acutely 
inflammation can be pro-angiogenic, but on a chronic systemic level, inflammation has been shown to negatively affect skeletal muscle and capillarity [158, 168, 183, 185, 187-189]. Inflammation, much like angiogenesis, is controlled by a complex interplay between many different inflammatory mediators. One of these mediators that is common between many of these diseases is tumor necrosis factor-alpha (TNF $\alpha$ ). TNF $\alpha$ has been shown to be a potent stimulator of the inflammatory process in vitro, in vivo, and has been implicated in the chronic systemic inflammation seen many chronic pathologies [156, 165, 186, 187, 190-195]. Interestingly, in COPD those patients with the most severe skeletal muscle dysfunction (muscle mass loss) also have the most severely elevated TNF $\alpha$ levels [190].

TSP-1 may serve as the axis by which TNF $\alpha$ decreases skeletal muscle capillarity in chronic pathology. It has been shown in vitro that increased TNF $\alpha$ increases TSP-1 in human skeletal muscle cells. This does not occur when the cells were treated with a variety of other inflammatory mediators [196], suggesting a TNF $\alpha$-TSP-1 specific interaction. This increase of TSP-1 was also associated with increases in both of the important TSP-1 receptors CD36 and CD47. Interestingly, it has also been shown that children with defects in the TNF $\alpha$ gene resulting in a higher TNF $\alpha$ expression also have increased circulating levels of TSP-1 [197]. $\mathrm{TNF} \alpha$ also has been shown to decrease striated (cardiac and skeletal) muscle mitochondrial function and density, potentially by multiple mechanism $[186,198,199]$. This has also been shown for TSP-1 [148]. It is plausible that the resultant increase in TSP-1 from increased TNF $\alpha$ could be the mechanism by which TNF $\alpha$ decreases mitochondrial density. Interestingly, TNF $\alpha$ KO animals also have increased running capacity [200], which leads to speculation that they have increased skeletal muscle function, and perhaps lower TSP-1 levels. Further, increased TSP-1 has actually been shown to increase TNF $\alpha$ in brain epithelial cells [201], which may 
suggest a feed-forward system. While this data together does not provide direct evidence for a TNF $\alpha$-TSP-1 axis, it does provide a potential mechanism for the decrease in capillarity seen in skeletal muscle of afore mentioned chronic diseases. 
Citations

1. Hood, D.A., et al., Coordination of metabolic plasticity in skeletal muscle. Journal of Experimental Biology, 2006. 209(12): p. 2265-2275.

2. Flück, M. and H. Hoppeler, Molecular basis of skeletal muscle plasticity-from gene to form and function, in Reviews of Physiology, Biochemistry and Pharmacology. 2003, Springer Berlin Heidelberg. p. 159-216.

3. Baldwin, K.M. and F. Haddad, Skeletal Muscle Plasticity: Cellular and Molecular Responses to Altered Physical Activity Paradigms. American Journal of Physical Medicine \& Rehabilitation, 2002. 81(11): p. S40-S51.

4. $\quad$ Pette, D., Historical Perspectives: Plasticity of mammalian skeletal muscle. Journal of Applied Physiology, 2001. 90(3): p. 1119-1124.

5. Flück, M., Functional, structural and molecular plasticity of mammalian skeletal muscle in response to exercise stimuli. Journal of Experimental Biology, 2006. 209(12): p. 22392248.

6. Harridge, S.D.R., Plasticity of human skeletal muscle: gene expression to in vivo function. Experimental physiology, 2007. 92(5): p. 783-797.

7. Marini, M. and A. Veicsteinas, The exercised skeletal muscle: a review. Eur J Translat Myology, 2010. 20(3): p. 105-20.

8. Andersen, P. and J. Henriksson, Capillary supply of the quadriceps femoris muscle of man: adaptive response to exercise. The Journal of Physiology, 1977. 270(3): p. 677-690.

9. Audet, G.N., et al., Expression of angiogenic regulators and skeletal muscle capillarity in selectively bred high aerobic capacity mice. Experimental physiology, 2011. 96(11): p. 1138-1150.

10. Gouzi, F., et al., Blunted muscle angiogenic training-response in copd patients versus sedentary controls. European Respiratory Journal, 2012.

11. Hoppeler, H. and E.R. Weibel, Limits for oxygen and substrate transport in mammals. Journal of Experimental Biology, 1998. 201(8): p. 1051-64.

12. Prior, B.M., H.T. Yang, and R.L. Terjung, What makes vessels grow with exercise training? Journal of Applied Physiology, 2004. 97(3): p. 1119-1128.

13. Richardson, R.S., et al., Determinants of maximal exercise VO2 during single leg kneeextensor exercise in humans. American Journal of Physiology - Heart and Circulatory Physiology, 1995. 268(4): p. H1453-H1461.

14. Roudier, E., et al., Angio-adaptation in unloaded skeletal muscle: new insights into an early and muscle type-specific dynamic process. The Journal of Physiology, 2010. 588(22): p. 4579-4591.

15. Tang, K., et al., Capillary regression in vascular endothelial growth factor-deficient skeletal muscle. Physiological Genomics, 2004. 18(1): p. 63-69.

16. Wong, L.E., et al., Anatomic capillarization is elevated in the medial gastrocnemius muscle of mighty mini mice. Journal of Applied Physiology, 2009. 106(5): p. 1660-1667.

17. Olfert, I.M., et al., Skeletal muscle capillarity and angiogenic mRNA levels after exercise training in normoxia and chronic hypoxia. Journal of Applied Physiology, 2001. 91(3): p. 1176-1184.

18. Olfert, I.M., et al., Myocyte vascular endothelial growth factor is required for exerciseinduced skeletal muscle angiogenesis. American Journal of Physiology - Regulatory, Integrative and Comparative Physiology, 2010. 299(4): p. R1059-R1067. 
19. Prior, B.M., et al., Exercise-induced vascular remodeling. Exerc Sport Sci Rev, 2003. 31(1): p. 26-33.

20. Waters, R.E., et al., Voluntary running induces fiber type-specific angiogenesis in mouse skeletal muscle. American Journal of Physiology - Cell Physiology, 2004. 287(5): p. C1342-C1348.

21. Wagner, P.D., Determinants of maximal oxygen transport and utilization. Annual review of physiology, 1996. 58(1): p. 21-50.

22. Malek, M.H. and I.M. Olfert, Global deletion of thrombospondin-1 increases cardiac and skeletal muscle capillarity and exercise capacity in mice. Experimental Physiology, 2009. 94(6): p. 749-760.

23. Olfert, I.M., et al., Muscle-specific VEGF deficiency greatly reduces exercise endurance in mice. The Journal of Physiology, 2009. 587(8): p. 1755.

24. Torre-Bueno, J., et al., Diffusion limitation in normal humans during exercise at sea level and simulated altitude. Journal of Applied Physiology, 1985. 58(3): p. 989-995.

25. Ahmetov, I., et al., Polymorphism of the vascular endothelial growth factor gene (VEGF) and aerobic performance in athletes. Human Physiology, 2008. 34(4): p. 477-481.

26. Wagner, P.D., Skeletal muscle angiogenesis. Hypoxia: From Genes to the Bedside (Roach, RC, Wagner, PD and Hackett, PH, eds.), 2001: p. 21-38.

27. Egginton, S., Invited review: activity-induced angiogenesis. Pflügers Archiv European Journal of Physiology, 2009. 457(5): p. 963-977.

28. Jobin, J., et al., Chronic obstructive pulmonary disease: capillarity and fiber-type characteristics of skeletal muscle. J Cardiopulm Rehabil, 1998. 18(6): p. 432-7.

29. Olfert, I.M. and O. Birot, Importance of Anti-angiogenic Factors in the Regulation of Skeletal Muscle Angiogenesis. Microcirculation, 2011. 18(4): p. 316-330.

30. Høier, B., et al., The effect of passive movement training on angiogenic factors and capillary growth in human skeletal muscle. The Journal of Physiology, 2010. 588(19): p. 3833-3845.

31. Chinsomboon, J., et al., The transcriptional coactivator PGC-1 \{alpha\} mediates exercise-induced angiogenesis in skeletal muscle. Science Signaling, 2009. 106(50): p. 21401.

32. Shweiki, D., et al., Vascular endothelial growth factor induced by hypoxia may mediate hypoxia-initiated angiogenesis. Nature, 1992. 359(6398): p. 843-845.

33. Clanton, T.L. and P.F. Klawitter, Invited review: Adaptive responses of skeletal muscle to intermittent hypoxia: the known and the unknown. Journal of Applied Physiology, 2001. 90(6): p. 2476-2487.

34. Egginton, S., et al., Physiological angiogenesis is a graded, not threshold, response. The Journal of Physiology, 2011. 589(1): p. 195-206.

35. Carmeliet, P., Mechanisms of angiogenesis and arteriogenesis. Nature medicine, 2000. 6(4): p. 389-395.

36. Milkiewicz, M., et al., Association between shear stress, angiogenesis, and VEGF in skeletal muscles in vivo. Microcirculation, 2001. 8(4): p. 229-241.

37. Fraisl, P., et al., Regulation of angiogenesis by oxygen and metabolism. Developmental cell, 2009. 16(2): p. 167-179.

38. Wahl, P., et al., Effects of acid-base balance and high or low intensity exercise on VEGF and $b F G F$. European journal of applied physiology, 2011. 111(7): p. 1405-1413. 
39. Audet, G.N., et al., Chronic delivery of a thrombospondin-1 mimetic decreases skeletal muscle capillarity in mice. PLoS One, 2013. 8(2): p. e55953.

40. $\quad$ Olfert, I.M., et al., Temporal thrombospondin-1 mRNA response in skeletal muscle exposed to acute and chronic exercise. Growth Factors, 2006. 24(4): p. 253-259.

41. Olfert, I.M., et al., Chronic hypoxia attenuates resting and exercise-induced VEGF, flt-1, and flk-1 mRNA levels in skeletal muscle. Journal of Applied Physiology, 2001. 90(4): p. 1532.

42. Dawson, D.W., et al., CD36 mediates the in vitro inhibitory effects of thrombospondin-1 on endothelial cells. The Journal of cell biology, 1997. 138(3): p. 707.

43. Kim, K.J., et al., Inhibition of vascular endothelial growth factor-induced angiogenesis suppresses tumour growth in vivo. 1993.

44. Miyazaki, Y., et al., Expression of a tumor necrosis factor-alpha transgene in murine lung causes lymphocytic and fibrosing alveolitis. A mouse model of progressive pulmonary fibrosis. J Clin Invest, 1995. 96(1): p. 250-9.

45. Müller, G., et al., Inhibitory action of transforming growth factor beta on endothelial cells. Proceedings of the National Academy of Sciences, 1987. 84(16): p. 5600-5604.

46. Tolsma, S.S., M.S. Stack, and N. Bouck, Lumen formation and other angiogenic activities of cultured capillary endothelial cells are inhibited by thrombospondin-1. Microvascular research, 1997. 54(1): p. 13-26.

47. Linda Vona-Davis, G.A., Sarah McLaughlin, and Mark Olfert, Basal VEGF protein expression in skeletal muscle, adipose tissue, and mammary tumors of PyMT mice. American Association of Cancer Research, 2012. 72(8).

48. Giles, F.J., The vascular endothelial growth factor (VEGF) signaling pathway: a therapeutic target in patients with hematologic malignancies. The Oncologist, 2001. 6(Supplement 5): p. 32.

49. Nagy, J.A., A.M. Dvorak, and H.F. Dvorak, VEGF-A and the induction of pathological angiogenesis. Annu. Rev. Pathol. Mech. Dis., 2007. 2: p. 251-275.

50. Neufeld, G., et al., Vascular endothelial growth factor (VEGF) and its receptors. The FASEB journal, 1999. 13(1): p. 9-22.

51. Nilsson, M. and J.V. Heymach, Vascular endothelial growth factor (VEGF) pathway. Journal of Thoracic Oncology, 2006. 1(8): p. 768.

52. Olsson, A.K., et al., VEGF receptor signalling? in control of vascular function. Nature Reviews Molecular Cell Biology, 2006. 7(5): p. 359-371.

53. Robinson, C.J. and S.E. Stringer, The splice variants of vascular endothelial growth factor (VEGF) and their receptors. Journal of cell science, 2001. 114(Pt 5): p. 853.

54. Simons, M., An Inside View: VEGF Receptor Trafficking and Signaling. Physiology, 2012. 27(4): p. 213-222.

55. Takahashi, H. and M. Shibuya, The vascular endothelial growth factor (VEGF)/VEGF receptor system and its role under physiological and pathological conditions. Clinical Science, 2005. 109: p. 227-241.

56. Kishlyansky, M., et al., Striated muscle angio-adaptation requires changes in Vasohibin1 expression pattern. Biochemical and biophysical research communications, 2010.

57. Mark Olfert, I. and O. Birot, Importance of anti angiogenic factors in the regulation of skeletal muscle angiogenesis. Microcirculation, 2011.

58. Carmeli, E., et al., Matrix metalloproteinases and skeletal muscle: a brief review. Muscle \& nerve, 2004. 29(2): p. 191-197. 
59. Burri, P.H. and V. Djonov, Intussusceptive angiogenesis - the alternative to capillary sprouting. Molecular aspects of medicine, 2002. 23(6): p. 1-27.

60. Burri, P.H., R. Hlushchuk, and V. Djonov, Intussusceptive angiogenesis: its emergence, its characteristics, and its significance. Developmental dynamics, 2004. 231(3): p. 474488.

61. Carmeliet, P., Angiogenesis in health and disease. Nature medicine, 2003. 9(6): p. 653660.

62. Plank, M. and B. Sleeman, Tumour-induced Angiogenesis: A Review: Review Article. Journal of Theoretical Medicine, 2003. 5(3-4): p. 137-153.

63. Folkman, J., Angiogenesis. Annu. Rev. Med., 2006. 57: p. 1-18.

64. Folkman, J. and Y. Shing, Angiogenesis. Journal of Biological Chemistry, 1992. 267(16): p. 10931-10934.

65. Moses, M.A., The regulation of neovascularization of matrix metalloproteinases and their inhibitors. Stem Cells, 1997. 15(3): p. 180-9.

66. Seandel, M., et al., Growth factor-induced angiogenesis in vivo requires specific cleavage of fibrillar type I collagen. Blood, 2001. 97(8): p. 2323-2332.

67. Haas, T., et al., Matrix metalloproteinase activity is required for activity-induced angiogenesis in rat skeletal muscle. American Journal of Physiology-Heart and Circulatory Physiology, 2000. 279(4): p. H1540-H1547.

68. Rullman, E., et al., A single bout of exercise activates matrix metalloproteinase in human skeletal muscle. Journal of Applied Physiology, 2007. 102(6): p. 2346-2351.

69. Hansen-Smith, F.M., O. Hudlicka, and S. Egginton, In vivo angiogenesis in adult rat skeletal muscle: early changes in capillary network architecture and ultrastructure. Cell and tissue research, 1996. 286(1): p. 123-136.

70. Makanya, A.N., R. Hlushchuk, and V.G. Djonov, Intussusceptive angiogenesis and its role in vascular morphogenesis, patterning, and remodeling. Angiogenesis, 2009. 12(2): p. 113-123.

71. Hoefer, I.E., B. den Adel, and M.J. Daemen, Biomechanical factors as triggers of vascular growth. Cardiovascular Research, 2013.

72. Kehler, D.S., N.S. Dhalla, and T.A. Duhamel, Biochemical Mechanisms of ExerciseInduced Angiogenesis, in Biochemical Basis and Therapeutic Implications of Angiogenesis. 2013, Springer. p. 181-206.

73. Tang, K., et al., Impaired exercise capacity and skeletal muscle function in a mouse model of pulmonary inflammation. J Appl Physiol, 2013. 114(9).

74. Breen, E.C., et al., Angiogenic growth factor $m R N A$ responses in muscle to a single bout of exercise. Journal of Applied Physiology, 1996. 81(1): p. 355-361.

75. Hanahan, D. and J. Folkman, Patterns and Emerging Mechanisms of the Angiogenic Switch during Tumorigenesis. Cell, 1996. 86(3): p. 353-364.

76. Neufeld, G., et al., Vascular endothelial growth factor (VEGF) and its receptors. The FASEB journal, 1999. 13(1): p. 9.

77. Bocci, G., et al., Thrombospondin 1, a mediator of the antiangiogenic effects of low-dose metronomic chemotherapy. Proceedings of the National Academy of Sciences of the United States of America, 2003. 100(22): p. 12917.

78. Bornstein, P., Thrombospondins function as regulators of angiogenesis. Journal of Cell Communication and Signaling, 2009. 3(3): p. 189-200. 
79. Good, D.J., et al., A tumor suppressor-dependent inhibitor of angiogenesis is immunologically and functionally indistinguishable from a fragment of thrombospondin. Proceedings of the National Academy of Sciences, 1990. 87(17): p. 6624-6628.

80. Lawler, J., The functions of thrombospondin-1 and-2. Current opinion in cell biology, 2000. 12(5): p. 634-640.

81. Lawler, J. and M. Detmar, Tumor progression: the effects of thrombospondin-1 and -2. The International Journal of Biochemistry \&amp; Cell Biology, 2004. 36(6): p. 10381045.

82. Gavin, T.P. and P.D. Wagner, Effect of short-term exercise training on angiogenic growth factor gene responses in rats. Journal of Applied Physiology, 2001. 90(4): p. 1219-1226.

83. Mosher, D.F., Physiology of thrombospondin. Annual review of medicine, 1990. 41(1): p. 85-97.

84. Jack, L., The functions of thrombospondin-1 and-2. Current Opinion in Cell Biology, 2000. 12(5): p. 634-640.

85. Chen, H., M.E. Herndon, and J. Lawler, The cell biology of thrombospondin-1. Matrix Biology, 2000. 19(7): p. 597-614.

86. Baenziger, N.L., G. Brodie, and P.W. Majerus, A thrombin-sensitive protein of human platelet membranes. Proceedings of the National Academy of Sciences, 1971. 68(1): p. 240-243.

87. Sargiannidou, I., J. Zhou, and G.P. Tuszynski, The role of thrombospondin-1 in tumor progression. Experimental Biology and Medicine, 2001. 226(8): p. 726-733.

88. Adams, J.C., Thrombospondin-1. The international journal of biochemistry \& cell biology, 1997. 29(6): p. 861-865.

89. Giancotti, F.G. and E. Ruoslahti, Integrin signaling. Science, 1999. 285(5430): p. 10281033.

90. Schwartz, M.A., Integrin signaling revisited. Trends in cell biology, 2001. 11(12): p. 466-470.

91. Somanath, P.R., A. Ciocea, and T.V. Byzova, Integrin and growth factor receptor alliance in angiogenesis. Cell biochemistry and biophysics, 2009. 53(2): p. 53-64.

92. Chandrasekaran, L., et al., Cell contact-dependent activation of alpha 3beta 1 integrin modulates endothelial cell responses to thrombospondin-1. Molecular Biology of the Cell, 2000. 11(9): p. 2885.

93. Chandrasekaran, S., et al., Pro-adhesive and Chemotactic Activities of Thrombospondin-1 for Breast Carcinoma Cells Are Mediated by $\alpha 3 \beta 1$ Integrin and Regulated by Insulin-like Growth Factor-1 and CD98. Journal of Biological Chemistry, 1999. 274(16): p. 1140811416.

94. Greenaway, J., et al., Thrombospondin-1 inhibits VEGF levels in the ovary directly by binding and internalization via the low density lipoprotein receptor-related protein-1 (LRP-1). Journal of cellular physiology, 2007. 210(3): p. 807-18.

95. Godyna, S., et al., Identification of the low density lipoprotein receptor-related protein (LRP) as an endocytic receptor for thrombospondin-1. The Journal of cell biology, 1995. 129(5): p. 1403-1410.

96. Short, S.M., et al., Inhibition of endothelial cell migration by thrombospondin-1 type-1 repeats is mediated by \{beta\}1 integrins. J. Cell Biol., 2005. 168(4): p. 643-653. 
97. Murphy-Ullrich, J.E., S. Schultz-Cherry, and M. Höök, Transforming growth factor-beta complexes with thrombospondin. Molecular Biology of the Cell, 1992. 3(2): p. 181.

98. Annes, J.P., J.S. Munger, and D.B. Rifkin, Making sense of latent TGF $\beta$ activation. Journal of cell science, 2003. 116(2): p. 217-224.

99. Sweetwyne, M.T. and J.E. Murphy-Ullrich, Thrombospondin1 in tissue repair and fibrosis: TGF- $\beta$-dependent and independent mechanisms. Matrix Biology, 2012. 31(3): p. 178-186.

100. Miao, W.-M., et al., Thrombospondin-1 Type 1 Repeat Recombinant Proteins Inhibit Tumor Growth through Transforming Growth Factor- $\beta$-dependent and-independent Mechanisms. Cancer research, 2001. 61(21): p. 7830-7839.

101. Schultz-Cherry, S., J. Lawler, and J.E. Murphy-Ullrich, The type 1 repeats of thrombospondin 1 activate latent transforming growth factor-beta. Journal of Biological Chemistry, 1994. 269(43): p. 26783-26788.

102. Korpal, M. and Y. Kang, Targeting the transforming growth factor- $\beta$ signalling pathway in metastatic cancer. European Journal of Cancer, 2010. 46(7): p. 1232-1240.

103. Derynck, R., R.J. Akhurst, and A. Balmain, TGF- $\beta$ signaling in tumor suppression and cancer progression. Nature genetics, 2001. 29(2): p. 117-129.

104. Bierie, B. and H.L. Moses, Tumour microenvironment: TGF $\beta$ : the molecular Jekyll and Hyde of cancer. Nature Reviews Cancer, 2006. 6(7): p. 506-520.

105. Pepper, M.S., Transforming growth factor-beta: vasculogenesis, angiogenesis, and vessel wall integrity. Cytokine \& growth factor reviews, 1997. 8(1): p. 21.

106. Christopher Richard Anderson, B.R.B., Richard J. Price, CD36 is Downregulated by VEGF-A and the Removal of Wall Shear Stress: Implications for the Regulation of a CD36- Capillary Sprout Specific Endothelial Phenotype. The FASEB Journal, 2007. 21: p. 897.20.

107. Febbraio, M., D.P. Hajjar, and R.L. Silverstein, CD36: a class B scavenger receptor involved in angiogenesis, atherosclerosis, inflammation, and lipid metabolism. Journal of Clinical Investigation, 2001. 108(6): p. 785-792.

108. Isenberg, J.S., C. Yu, and D.D. Roberts, Differential effects of ABT-510 and a CD36binding peptide derived from the type 1 repeats of thrombospondin-1 on fatty acid uptake, nitric oxide signaling, and caspase activation in vascular cells. Biochemical Pharmacology, 2008. 75(4): p. 875-882.

109. Jiménez, B., et al., Signals leading to apoptosis-dependent inhibition of neovascularization by thrombospondin-1. Nature medicine, 2000. 6(1): p. 41-48.

110. Primo, L., et al., Identification of CD36 molecular features required for its in vitro angiostatic activity. The FASEB journal, 2005. 19(12): p. 1713-1715.

111. Tsuchida, T., et al., Expression of the thrombospondin 1 receptor CD36 is correlated with decreased stromal vascularisation in colon cancer. International journal of oncology, 1999. 14(1): p. 47-52.

112. Zhang, X., et al., Thrombospondin-1 modulates vascular endothelial growth factor activity at the receptor level. The FASEB journal, 2009. 23(10): p. 3368-3376.

113. Greenwalt, D.E., et al., Membrane glycoprotein CD36: a review of its roles in adherence, signal transduction, and transfusion medicine. Blood, 1992. 80(5): p. 1105-1115.

114. Guo, N., et al., Thrombospondin 1 and type I repeat peptides of thrombospondin 1 specifically induce apoptosis of endothelial cells. Cancer research, 1997. 57(9): p. 1735. 
115. Streit, M., et al., Overexpression of Thrombospondin-1 Decreases Angiogenesis and Inhibits the Growth of Human Cutaneous Squamous Cell Carcinomas. The American Journal of Pathology, 1999. 155(2): p. 441-452.

116. Campbell, N.E., et al., The Thrombospondin-1 Mimetic ABT-510 Increases the Uptake and Effectiveness of Cisplatin and Paclitaxel in a Mouse Model of Epithelial Ovarian Cancer, Neoplasia Press Inc.

117. Reiher, F.K., et al., Inhibition of tumor growth by systemic treatment with thrombospondin-1 peptide mimetics. International Journal of Cancer, 2002. 98(5): p. 682689.

118. Gupta, K., et al., Binding and displacement of vascular endothelial growth factor (VEGF) by thrombospondin: Effect on human microvascular endothelial cell proliferation and angiogenesis. Angiogenesis, 1999. 3(2): p. 147-158.

119. Inoki, I., et al., Connective tissue growth factor binds vascular endothelial growth factor (VEGF) and inhibits VEGF-induced angiogenesis. The FASEB journal, 2002. 16(2): p. 219-221.

120. Zhang, X., et al., Thrombospondin-1 modulates vascular endothelial growth factor activity at the receptor level. The FASEB journal, 2009. 23(10): p. 3368.

121. Greenaway, J., et al., ABT-510 induces tumor cell apoptosis and inhibits ovarian tumor growth in an orthotopic, syngeneic model of epithelial ovarian cancer. Molecular cancer therapeutics, 2009. 8(1): p. 64.

122. Kaur, S., et al., Thrombospondin-1 Inhibits VEGF Receptor-2 Signaling by Disrupting Its Association with CD47. Journal of Biological Chemistry, 2010. 285(50): p. 38923-38932.

123. Shibuya, M., Differential roles of vascular endothelial growth factor receptor-1 and receptor-2 in angiogenesis. Journal of biochemistry and molecular biology, 2006. 39(5): p. 469.

124. Dawson, D.W., et al., Three distinct D-amino acid substitutions confer potent antiangiogenic activity on an inactive peptide derived from a thrombospondin-1 type 1 repeat. Molecular pharmacology, 1999. 55(2): p. 332-338.

125. Anderson, J.C., et al., ABT-510, a modified type 1 repeat peptide of thrombospondin, inhibits malignant glioma growth in vivo by inhibiting angiogenesis. Cancer biology \& therapy, 2007. 6(3): p. 454.

126. Hasina, R., et al., ABT-510 is an effective chemopreventive agent in the mouse 4nitroquinoline 1-oxide model of oral carcinogenesis. Cancer Prevention Research, 2009. 2(4): p. 385.

127. Hoekstra, R., et al., Phase I Safety, Pharmacokinetic, and Pharmacodynamic Study of the Thrombospondin-1-Mimetic Angiogenesis Inhibitor ABT-510 in Patients With Advanced Cancer. Journal of Clinical Oncology, 2005. 23(22): p. 5188-5197.

128. Joyce, D., et al., Evaluation of the Thrombospondin-1 analogue ABT-510 in the APCMin/+ mouse intestinal adenoma model. Journal of Clinical Oncology, 2006. 24(18_suppl): p. 13545.

129. Rusk, A., et al., Preclinical evaluation of antiangiogenic thrombospondin-1 peptide mimetics, ABT-526 and ABT-510, in companion dogs with naturally occurring cancers. Clinical cancer research, 2006. 12(24): p. 7444.

130. Yap, R., et al., Metronomic low-dose chemotherapy boosts CD95-dependent antiangiogenic effect of the thrombospondin peptide ABT-510: a complementation antiangiogenic strategy. Clinical cancer research, 2005. 11(18): p. 6678. 
131. Hoekstra, R., et al., Phase I safety, pharmacokinetic, and pharmacodynamic study of the thrombospondin-1-mimetic angiogenesis inhibitor ABT-510 in patients with advanced cancer. Journal of Clinical Oncology, 2005. 23(22): p. 5188-5197.

132. Isenberg, J.S., D.D. Roberts, and W.A. Frazier, CD47: a new target in cardiovascular therapy. Arteriosclerosis, thrombosis, and vascular biology, 2008. 28(4): p. 615.

133. Brown, E.J. and W.A. Frazier, Integrin-associated protein (CD47) and its ligands. Trends in cell biology, 2001. 11(3): p. 130-135.

134. Lindberg, F.P., et al., Decreased resistance to bacterial infection and granulocyte defects in IAP-deficient mice. Science (New York, NY), 1996. 274(5288): p. 795.

135. Zhang, X. and J. Lawler, Thrombospondin-based antiangiogenic therapy. Microvascular research, 2007. 74(2-3): p. 90-99.

136. Isenberg, J.S., et al., CD47 is necessary for inhibition of nitric oxide-stimulated vascular cell responses by thrombospondin-1. Journal of Biological Chemistry, 2006. 281(36): p. 26069-26080.

137. Xing, C., et al., Neurovascular effects of CD47 signaling: promotion of cell death, inflammation, and suppression of angiogenesis in brain endothelial cells in vitro. Journal of neuroscience research, 2009. 87(11): p. 2571-2577.

138. Isenberg, J.S., et al., Thrombospondin-1 inhibits endothelial cell responses to nitric oxide in a cGMP-dependent manner. Proceedings of the National Academy of Sciences of the United States of America, 2005. 102(37): p. 13141.

139. Ridnour, L.A., et al., Nitric oxide regulates angiogenesis through a functional switch involving thrombospondin-1. Proceedings of the National Academy of Sciences of the United States of America, 2005. 102(37): p. 13147-13152.

140. Roberts, D.D., et al., The matricellular protein thrombospondin-1 globally regulates cardiovascular function and responses to stress via CD47. Matrix Biology, 2012.

141. Stamler, J.S. and G. Meissner, Physiology of nitric oxide in skeletal muscle. Physiological Reviews, 2001. 81(1): p. 209-237.

142. Ziche, M., et al., Nitric oxide mediates angiogenesis in vivo and endothelial cell growth and migration in vitro promoted by substance P. Journal of Clinical Investigation, 1994. 94(5): p. 2036.

143. Ziche, M. and L. Morbidelli, Nitric oxide and angiogenesis. Journal of neuro-oncology, 2000. 50(1-2): p. 139-148.

144. Ramanathan, S., et al., Thrombospondin-1 and Angiotensin II Inhibit Soluble Guanylyl Cyclase through an Increase in Intracellular Calcium Concentration. Biochemistry, 2011. 50(36): p. 7787-7799.

145. Watkins, S.C., et al., Thrombospondin expression in traumatized skeletal muscle. Cell and tissue research, 1990. 261(1): p. 73-84.

146. Kivela, R., et al., Exercise-induced expression of angiogenic growth factors in skeletal muscle and in capillaries of healthy and diabetic mice. Cardiovascular Diabetology, 2008. 7(1): p. 13.

147. Kivelä, R., et al., Effects of experimental type 1 diabetes and exercise training on angiogenic gene expression and capillarization in skeletal muscle. The FASEB journal, 2006. 20(9): p. 1570-1572.

148. Frazier, E.P., et al., Age-dependent regulation of skeletal muscle mitochondria by the thrombospondin-1 receptor CD47. Matrix Biology, 2011. 
149. Miniño, A.M. and S.L. Murphy, Death in the United States, 2010. NCHS data brief, 2012(99): p. 1-8.

150. Ward, M., et al., Direct medical cost of chronic obstructive pulmonary disease in the USA. Respiratory medicine, 2000. 94(11): p. 1123-1129.

151. Thorpe, K.E. and M. Philyaw, The medicalization of chronic disease and costs. Annual review of public health, 2012. 33: p. 409-423.

152. Durstine, J.L., K. Burns, and R. Cheek, Physical Activity in Treatment of Chronic Conditions. Physical Activity and Public Health Practice, 2012: p. 93.

153. Zhang, P., et al., Global healthcare expenditure on diabetes for 2010 and 2030. Diabetes research and clinical practice, 2010. 87(3): p. 293.

154. Finkelstein, E.A., et al., Annual medical spending attributable to obesity: payer-and service-specific estimates. Health affairs, 2009. 28(5): p. w822-w831.

155. Chung, K.F., Inflammatory mediators in chronic obstructive pulmonary disease. Current Drug Targets-Inflammation \& Allergy, 2005. 4(6): p. 619-625.

156. Dandona, P., A. Aljada, and A. Bandyopadhyay, Inflammation: the link between insulin resistance, obesity and diabetes. Trends in immunology, 2004. 25(1): p. 4-7.

157. Danesh, J., et al., Low grade inflammation and coronary heart disease: prospective study and updated meta-analyses. BMJ, 2000. 321(7255): p. 199-204.

158. Dourado, V.Z., et al., Systemic manifestations in chronic obstructive pulmonary disease. J Bras Pneumol, 2006. 32(2): p. 161-71.

159. Duncan, B.B., et al., Low-Grade Systemic Inflammation and the Development of Type 2 Diabetes. Diabetes, 2003. 52(7): p. 1799-1805.

160. Gan, W., et al., Association between chronic obstructive pulmonary disease and systemic inflammation: a systematic review and a meta-analysis. Thorax, 2004. 59(7): p. 574-580.

161. Sattar, N., et al., Explaining how "high-grade" systemic inflammation accelerates vascular risk in rheumatoid arthritis. Circulation, 2003. 108(24): p. 2957-2963.

162. Vgontzas, A.N., et al., Chronic systemic inflammation in overweight and obese adults. JAMA: the journal of the American Medical Association, 2000. 283(17): p. 2235-2236.

163. Wellen, K.E. and G.S. Hotamisligil, Inflammation, stress, and diabetes. The Journal of Clinical Investigation, 2005. 115(5): p. 1111-1119.

164. $\mathrm{Xu}, \mathrm{H}$., et al., Chronic inflammation in fat plays a crucial role in the development of obesity-related insulin resistance. The Journal of Clinical Investigation, 2003. 112(12): p. 1821-1830.

165. Gosker, H.R., et al., Striking Similarities in Systemic Factors Contributing to Decreased Exercise Capacity in Patients With Severe Chronic Heart Failure or COPD*. Chest, 2003. 123(5): p. 1416-1424.

166. Hülsmann, M., et al., Muscle strength as a predictor of long-term survival in severe congestive heart failure. European Journal of Heart Failure, 2004. 6(1): p. 101-107.

167. Burtin, C., et al., Effectiveness of exercise training in patients with COPD: the role of muscle fatigue. Eur Respir J, 2012. 40(2): p. 338-44.

168. Casaburi, R., Skeletal Muscle Function in COPD*. Chest, 2000. 117(5 suppl 1): p. 267S271S.

169. Clark, C., et al., Skeletal muscle strength and endurance in patients with mild COPD and the effects of weight training. European Respiratory Journal, 2000. 15(1): p. 92-97.

170. Man, W., et al., Skeletal muscle dysfunction in COPD: clinical and laboratory observations. Clinical Science, 2009. 117: p. 251-264. 
171. Serres, I., et al., Impaired skeletal muscle endurance related to physical inactivity and altered lung function in COPD patients. Chest, 1998. 113(4): p. 900-5.

172. Wouters, E.F.M., E.C. Creutzberg, and A.M.W.J. Schols, SYstemic effects in copd*. CHEST Journal, 2002. 121(5_suppl): p. 127S-130S.

173. Zuo, L., L. Nogueira, and M.C. Hogan, Effect of pulmonary TNF-alpha overexpression on mouse isolated skeletal muscle function. Am J Physiol Regul Integr Comp Physiol, 2011. 301(4): p. R1025-31.

174. Duscha, B.D., et al., Capillary density of skeletal muscle: A contributing mechanism for exercise intolerance in class II-III chronic heart failure independent of other peripheral alterations. Journal of the American College of Cardiology, 1999. 33(7): p. 1956-1963.

175. Harrington, D., et al., Skeletal Muscle Function and Its Relation to Exercise Tolerance in Chronic Heart Failure. Journal of the American College of Cardiology, 1997. 30(7): p. 1758-1764.

176. Hurley, B.F., E.D. Hanson, and A.K. Sheaff, Strength training as a countermeasure to aging muscle and chronic disease. Sports Medicine, 2011. 41(4): p. 289-306.

177. Coats, A.J., Research on cachexia, sarcopenia and skeletal muscle in cardiology. Journal of cachexia, sarcopenia and muscle, 2012. 3(4): p. 219-223.

178. Tisdale, M.J., Cachexia in cancer patients. Nature Reviews Cancer, 2002. 2(11): p. 862871.

179. Troosters, T., et al., Skeletal muscle weakness, exercise tolerance and physical activity in adults with cystic fibrosis. European Respiratory Journal, 2009. 33(1): p. 99-106.

180. Adams, G.R. and N.D. Vaziri, Skeletal muscle dysfunction in chronic renal failure: effects of exercise. American Journal of Physiology-Renal Physiology, 2006. 290(4): p. F753-F761.

181. Wells, G.D., et al., Skeletal muscle metabolic dysfunction in obesity and metabolic syndrome. The Canadian Journal of Neurological Sciences, 2008. 35(1): p. 31-40.

182. Rogers, M.A. and W.J. Evans, Changes in skeletal muscle with aging: effects of exercise training. Exercise and sport sciences reviews, 1993. 21(1): p. 65-102.

183. Degens, H. and S.E. Alway, Control of muscle size during disuse, disease, and aging. International journal of sports medicine, 2006. 27(2): p. 94-99.

184. Marcell, T.J., Review article: Sarcopenia: causes, consequences, and preventions. The Journals of Gerontology Series A: Biological Sciences and Medical Sciences, 2003. 58(10): p. M911-M916.

185. Barreiro, E., et al., Cytokine profile in quadriceps muscles of patients with severe COPD. Thorax, 2008. 63(2): p. 100-107.

186. Gosker, H.R., et al., Skeletal muscle dysfunction in chronic obstructive pulmonary disease and chronic heart failure: underlying mechanisms and therapy perspectives. The American Journal of Clinical Nutrition, 2000. 71(5): p. 1033-1047.

187. Fajardo, L.F., et al., Dual role of tumor necrosis factor-alpha in angiogenesis. The American Journal of Pathology, 1992. 140(3): p. 539.

188. Halin, C. and M. Detmar, Inflammation, angiogenesis, and lymphangiogenesis. Methods in enzymology, 2008. 445: p. 1-25.

189. Costa, C., J. Incio, and R. Soares, Angiogenesis and chronic inflammation: cause or consequence? Angiogenesis, 2007. 10(3): p. 149-166.

190. Di Francia, M., et al., Tumor necrosis factor-alpha levels and weight loss in chronic obstructive pulmonary disease. Am J Respir Crit Care Med, 1994. 150(5 Pt 1): p. 1453-5. 
191. Takabatake, N., et al., Circulating leptin in patients with chronic obstructive pulmonary disease. Am J Respir Crit Care Med, 1999. 159(4 Pt 1): p. 1215-9.

192. Yamakawa, I., et al., Inactivation of TNF- $\alpha$ ameliorates diabetic neuropathy in mice. American Journal of Physiology - Endocrinology And Metabolism, 2011. 301(5): p. E844-E852.

193. Tracey, K.J. and A. Cerami, Tumor necrosis factor, other cytokines and disease. Annual review of cell biology, 1993. 9(1): p. 317-343.

194. Van Dullemen, H.M., et al., Treatment of Crohn's disease with anti-tumor necrosis factor chimeric monoclonal antibody (cA2). Gastroenterology, 1995. 109(1): p. 129-135.

195. Levine, B., et al., Elevated circulating levels of tumor necrosis factor in severe chronic heart failure. New England Journal of Medicine, 1990. 323(4): p. 236-241.

196. Salajegheh, M., et al., Upregulation of thrombospondin-1 (TSP-1) and its binding partners, CD36 and CD47, in sporadic inclusion body myositis. Journal of neuroimmunology, 2007. 187(1-2): p. 166-174.

197. Lutz, J., et al., Increased Plasma Thrombospondin-1 (TSP-1) Levels Are Associated with the TNF $\alpha-308 A$ Allele in Children with Juvenile Dermatomyositis. Clinical Immunology, 2002. 103(3): p. 260-263.

198. Moe, G.W., et al., In vivo TNF- $\alpha$ inhibition ameliorates cardiac mitochondrial dysfunction, oxidative stress, and apoptosis in experimental heart failure. American Journal of Physiology-Heart and Circulatory Physiology, 2004. 287(4): p. H1813-H1820.

199. Valerio, A., et al., TNF- $\alpha$ downregulates eNOS expression and mitochondrial biogenesis in fat and muscle of obese rodents. The Journal of Clinical Investigation, 2006. 116(10): p. 2791-2798.

200. Netea, M.G., et al., Increased voluntary exercise in mice deficient for tumour necrosis factor- $\alpha$ and lymphotoxin- $\alpha$. European Journal of Clinical Investigation, 2007. 37(9): p. 737-741.

201. Rege, T.A., et al., Thrombospondin-1 -induced apoptosis of brain microvascular endothelial cells can be mediated by TNF-R1. Journal of cellular physiology, 2009. 218(1): p. 94-103. 


\title{
Chapter 3: Chronic delivery of a thrombospondin-1 mimetic decreases skeletal muscle capillarity in mice
}

\author{
As published in PLoS One, \\ February 2013, Volume 8, Issue 2 \\ PMID: 23405239
}


Chronic delivery of a thrombospondin-1 mimetic decreases skeletal muscle capillarity in mice

Gerald N. Audet, Daniel Fulks, Janelle C. Stricker, and I. Mark Olfert

West Virginia University School of Medicine, Center for Cardiovascular and Respiratory

Sciences, Division of Exercise Physiology, Morgantown, WV, USA

Address for correspondence:

Mark Olfert, $\mathrm{PhD}$

West Virginia University School of Medicine

Center for Cardiovascular and Respiratory Sciences

Division of Exercise Physiology

PO Box 9105

Morgantown, WV 26508

Phone 304-293-7597

molfert@hsc.wvu.edu 


\section{Abstract}

Angiogenesis is an essential process for normal skeletal muscle function. There is a growing body of evidence suggesting that thrombospondin-1 (TSP-1), a potent antiangiogenic protein in tumorigenesis, is an important regulator of both physiological and pathological skeletal muscle angiogenesis. We tested the hypothesis that chronic exposure to a TSP-1 mimetic (ABT-510), which targets the CD36 TSP-1 receptor, would decrease skeletal muscle capillarity as well as alter the balance between positive and negative angiogenic proteins under basal conditions. Osmotic minipumps with either ABT-510 or vehicle (5\% dextrose) were implanted subcutaneously in the subscapular region of C57/BL6 mice for 14 days. When compared to the vehicle treated mice, the ABT-510 group had a 20\% decrease in capillarity in the superficial region of the gastrocnemius (GA), 11\% decrease in the plantaris (PLT), and a 35\% decrease in the soleus (SOL). ABT-510 also decreased muscle protein expression of vascular endothelial growth factor (VEGF) in both the GA $(-140 \%)$ and SOL (-62\%); however there was no change in VEGF in the PLT. Serum VEGF was not altered in ABT-510 treated animals. Endogenous TSP-1 protein expression in all muscles remained unaltered. Tunnel staining revealed no difference in muscle apoptosis between ABT-510 and vehicle treated groups. These data provide evidence that the anti-angiogenic effects of TSP-1 are mediated, at least in part, via the CD36 receptor. It also suggests that under physiologic conditions the TSP-1/CD36 axis plays a role in regulating basal skeletal muscle microvessel density. 


\section{Introduction}

Physiologic angiogenesis and homeostasis of adult blood vessels is a complex process that is highly regulated by a balance between positive and negative angiogenic proteins. While there is a substantial body of evidence on the role positive angiogenic factors, such as vascular endothelial growth factor (VEGF) [1,2,3], the role of negative angiogenic factors is incomplete and poorly understood [4].

There are a number of known angiogenic inhibitors, of which thrombospondin-1 (TSP-1) is thought to play a prominent role in skeletal muscle angiogenesis [4]. TSP-1 is a large (450kD) extracellular protein that has a wide array of functions [5]. First discovered for its role in wound healing and platelet activation, it also has important roles in apoptosis, inflammation, nitric oxide signaling, and inhibition of positive angiogenic proteins $[6,7,8,9,10]$. Acting through CD36 (one of its receptors), TSP-1 has been shown to prevent endothelial cell adhesion, growth, and migration, as well as increase apoptosis $[11,12,13]$. The anti-angiogenic effects of TSP-1 in cancer pathology are well established. For example, a reduction in TSP-1 has been shown to increase tumor vessel growth, whereas pharmacological administration of several different TSP1 mimetics has helped decrease tumor size and disease progression in animal models $[14,15,16,17]$

Under physiologic conditions, TSP-1 expression has been shown to be responsive to exercise $[18,19]$, which suggests that TSP-1 may play a role in regulating exercise-induced skeletal muscle angiogenesis. TSP-1 KO mice have elevated skeletal muscle capillarity compared to wild-type mice, suggesting a critical role for TSP-1 in the physiological maintenance of capillaries [4]. Evidence in rats also shows that hindlimb unloading increases TSP-1 in association with decreases in skeletal muscle capillarity [3]. Recently, TSP-1 and it's 
receptor CD47 have also been suggested to play a pivotal role in skeletal muscle mitochondrial biogenesis, and therefore it is likely to be important in overall skeletal muscle function and adaptation to exercise stress [20].

Given the growing data implicating that importance of TSP-1 as a key angiogenic regulator in skeletal muscle, we sought to determine the consequences of chronically stimulating the TSP-1 pathway using ABT-510. ABT-510 is a mimetic of the conserved type I repeat region of TSP-1 which has been shown previously to inhibit angiogenesis through the CD36 receptor $[12,13,21]$. ABT-510 has been synthesized to have a longer half life in circulation than the native type I repeats of TSP-1 which allows for increased chronic stimulation of the TSP-1/CD36 pathway [22]. ABT-510 has been shown to be a potent inhibitor of vascular growth in tumorigenesis both in vitro and in vivo [15,23,24,25,26,27,28], which has been attributed to its binding to the CD36 receptor. Using this peptide we aimed to examine whether increased stimulation of the CD36 arm of the TSP-1 pathway affected skeletal muscle structure and function. Given that TSP-1 is a negative angiogenic regulator, we hypothesized that mice subjected to the ABT-510 would have lower skeletal muscle capillarity, mirrored by an altered balance between positive and negative angiogenic proteins.

\section{Methods}

\section{$\underline{\text { Animals }}$}

This study used 10-12 week old male C57BL/6 mice purchased from Jackson Laboratories (Strain no. 000664, C57BL/6J, Bar Harbor, ME). Mice were randomly selected to be placed in two groups (control, $n=8$; experimental $n=8$ ). All procedures that involved animals were approved by the West Virginia University Institutional Animal Care and Use Committee. 


\section{Osmotic Pump Drug Delivery}

The TSP-1 mimetic ABT-510 was generously provided by Abbott (Abbott, IL). This compound was chosen for use in this study based on previous and preliminary work showing its effectiveness in decreasing capillarity in tumors, both in vitro and in vivo [15,22,23,24,25,29,30]. ABT-510 was dissolved in 5\% dextrose (vehicle) over a 48 hour period to ensure the compound was fully dissolved, and then inserted into mini-osmotic pumps (Model 1002, Alzet Osmotic Pumps, Cupertino, CA,). Pumps were filled with either the drug or vehicle solution 24 hours in advance of implantation as per manufacture instructions. We delivered the maximum concentration of $30 \mathrm{mg} / \mathrm{kg} /$ day based on the maximum pump capacity $(100 \mu \mathrm{L})$, the mass of the animals, and the solubility of the drug (ABT-510 solubility curves [22]). Pumps were surgically inserted (flow-moderator first) subcutaneously in the scapular region while the mice were under anesthesia ( $2 \%$ isoflurane). The surgical site was disinfected with iodine and closed using sutures. Animals were then housed individually and observed daily for the remainder of the study for pain or distress. Pumps remained in the animals for 14 days, after which they were sacrificed and tissue/organ samples were collected and stored at $-80^{\circ}$ for later analysis.

\section{Morphometry}

Hindlimb skeletal muscle was surgically excised and frozen in isopentane cooled by liquid nitrogen. Frozen tissue was cut using a $-20^{\circ} \mathrm{C}$ cyromicrotome (Jung-Reichert Cryocut 1800: Cambridge Instruments, Germany) to yield $10 \mu \mathrm{m}$ transverse sections. Great care was taken to ensure the cryosectioned muscles were cut along the transverse plane. Sections were stained for dipeptidly-peptidase IV (DPP IV) and alkaline phosphatase (AP) following the method of Lojda (1979), as applied to skeletal muscle tissue [31,32]. A light microscope (Zeiss primo star, Zeiss, Oberkochen, Germany) was used to digitally acquire (Axiocam IC c3, Axiovision 4.8.2.0, Zeiss, 
Oberkochen, Germany) 20X images of the gastrocnemius, plantaris, and soleus. Capillary and myofiber counting was performed by a single individual blinded to group identification. For the gastrocnemius muscle, we obtained images in a checkerboard fashion across the entire muscle, thus both superficial and deep regions within the gastrocnemius could be included in the analysis. For the plantaris and soleus muscles, respectively, the entire muscle was imaged and analyzed. Counting was performed by visualization from acquired images using a custom program in MATLAB (version 7.0.0.27, The Mathworks, MA, USA) allowing the operator to visually mark and count the capillaries and fibers on each image. Capillary-to-fiber ratio (C:F,

number of capillaries/number muscle fibers), capillary density (CD, number of capillaries/mm ${ }^{2}$ muscle fiber area), and fiber cross sectional area (FCSA) were separately calculated for the gastrocnemius (GA), soleus (SOL), and plantaris (PLT) $(n=33-101$ images/muscle/group).

\section{$\underline{\text { Protein Analysis }}$}

GA, SOL, and PLT muscles from each group were excised and flash frozen in liquid nitrogen. They were then separately homogenized in a lysis buffer containing $50 \mathrm{mM}$ Tris/HCl (pH 7.4), 150mM NaCl, 0.5\% Triton X-100, and protease inhibitors (Complete Tablet, Roche Applied Science, Indianapolis, IN). Homogenates were centrifuged at $4^{\circ} \mathrm{C}$, at $8,000 \mathrm{~g}$ for 10 minutes, and supernatants removed and placed in new tubes. Blood samples were obtained from the heart and allowed to coagulate on ice. They were then centrifuged at $3000 \mathrm{~g}$ for 10 minutes and flash frozen in liquid nitrogen. Total protein was measured by bradford assay (\#23236 Pierce Coomassie Plus Protein Assay Kit, Thermo Scientific, Rockford, IL).

Quantification of VEGF was made from a total of $100 \mu \mathrm{g}$ of protein using a commercially available ELISA kit according to the manufactures instructions (\# MMV00, R\&D Systems, Minneapolis, MN, USA). Quantification of VEGFR-2 and P-VEGFR-2 were made from a total 
of $100 \mu \mathrm{g}$ of protein using a commercially available ELISA kit according to the manufactures instructions (\# 7335S, \#7340S, Cell Signaling, Danvers, MA, USA). TSP-1 and CD36 were analyzed via western blot. In brief, samples were separated on a 3-8\% SDS-PAGE (NuPAGE Novex 3-8\% Tris-Acetate Midi Gel, Invitrogen, Burlington, ON, Canada) and blotted onto a 0.45 $\mu \mathrm{m}$ nitrocellulose membrane (Pierce nitrocellulose membrane, Thermo Scientific, Rockford, IL). After blocking with 5\% fat-free milk, membranes were probed using antibodies against TSP-1 (1:1000, clone A6.1, \#399300, Invitrogen, Burlington, ON, Canada), $\beta$-tubulin (1:1000, \#2148, Cell Signaling), CD36 (1:250 \#552544, BD Pharmingen, Franklin Lakes, NJ, USA), secondary HRP-conjugated anti-mouse (1:1000, \#p0260, Dako, GE Healthcare, Piscataway, NJ) and secondary HRP-conjugated anti-rabbit (1:1000, \#p0217, Dako). Proteins were visualized using chemiluminescence detection (Pierce ECL, Thermo Scientific, Rockford, IL) and digitally imaged (G:BOX Gel imager, Syngene, Cambridge, UK) using Genesnap software (Ver. 7.01, Syngene, Cambridge, UK). Equal protein loading was verified by immunodetection of $\beta$-tubulin as our loading control. Quantification of protein expression levels were carried out using NIH Image J Software (v1.62) and expressed as densitometric arbitrary units (AU).

\section{$\underline{\text { Apoptosis }}$}

Nuclei exhibiting apoptotic changes were identified by TdT-mediated dUTP nick end labeling (TUNEL) according to the manufacturer's recommendations (Roche Molecular Biochemicals, Pleasanton, CA). Briefly, muscle cross sections were cut on a cryostat $(10 \mu \mathrm{m})$ and fixed in $4 \%$ paraformaldehyde at room temperature, blocked in 3\% $\mathrm{H} 2 \mathrm{O} 2$ in $100 \%$ methanol at room temperature, and permeabilized in $0.1 \%$ Triton $\mathrm{X}$ and $0.1 \%$ sodium citrate. TUNEL reaction mix was added in a 1:7.5 dilution, and the sections were incubated at $37^{\circ} \mathrm{C}$ for $1 \mathrm{~h}$. Sections were reacted with fluorescein antibody for $30 \mathrm{~min}$ at $37^{\circ} \mathrm{C}$, and substrate was added for 
color development. To control for false-positives, samples were then counter stained by DAPI staining to identify the nucleus. TUNEL staining was performed using a fluorescein TUNEL kit at 1:7.5 dilution as recommended by the manufacturer (Roche Molecular Biochemicals, Pleasanton, CA). Positive nuclei were counted, and at high power (x400), it was determined whether they were associated with the myofiber or with the interstitial space. The number of positive nuclei is expressed per whole muscle section. Positive controls were created using a DNase treatment of 1 serial section (\# AM1906, DNA-free, Ambion, Austin, TX). Negative controls were created by staining 1 serial section only for DAPI without TUNEL staining. This resulted in each sample containing 1 negative, 1 positive, and 2 experimental sections per slide.

\section{$\underline{\text { Statistics }}$}

All data are presented as means +/- SEM. To examine body mass, organ masses, muscle capillarity and molecular responses we used a student's T-test. A repeated measures ANOVA was used to analyze the maximal running test. An alpha level at $P<0.05$ was selected for statistical significance.

\section{Results}

\section{Exercise Testing and Morphometry}

Body and Muscle Mass: There was no significant difference in the absolute body or individual hindlimb muscle masses between ABT-510 (mimetic) and vehicle treated groups (Table 1). Heart and skeletal muscle normalized to body mass were not different between the groups (Table 1). .

Maximal Running Test: There was no difference in maximal running speed between vehicle and mimetic treated animals pre- or post-treatment. 
Morphometry: In the superficial region of the gastrocnemius (GA) muscle there was a $20 \%$ decrease in $\mathrm{C}: \mathrm{F}(\mathrm{P} \leq 0.05)$ and a similar trend in $\mathrm{CD}(25 \%$ decrease, $\mathrm{P}=0.055)$ in the mimetic group compared to vehicle (Figure $1 \& 2$ ). There was no statistical difference in C:F of the deep portion of the GA between groups, however there was a significant $25 \%$ decrease in CD $(\mathrm{P} \leq 0.05)$. FCSA was not significantly different between groups in either portions of the GA.

In the plantaris (PLT) muscle, there was a 11\% decrease in the C:F in the PLT in the mimetic group compared to vehicle ( $\mathrm{P} \leq 0.05)$ (Figure 3 ). There was no difference in $\mathrm{CD}$ or FCSA in the PLT.

In the soleus (SOL) muscle, there was a 35\% decrease in the C:F in the SOL in the mimetic group compared to vehicle $(\mathrm{P} \leq 0.01)$ (Figure 3$)$. There was no difference in $\mathrm{CD}$ $(\mathrm{P}=0.08)$ or FCSA $(\mathrm{P}=0.09)$.

\section{Endogenous VEGF, VEGFR-2, p-VEGFR-2, TSP-1, and CD36 Levels}

Skeletal muscle VEGF protein expression was decreased by $147 \%$ and $62 \%$ in the GA and SOL respectively $(\mathrm{P} \leq 0.05$, Figure 4$)$. In contrast, no significant change in VEGF protein expression was seen in the in the PLT. Serum VEGF levels were unchanged between ABT-510 and vehicle treated animals.

Total VEGFR-2 and phorphylated-VEGFR-2 (p-VEGFR-2) levels were also assessed in the GA. There was no difference in total VEGFR-2 levels, p-VEGFR-2 levels, or a ratio of VEGF-R2/p-VEGF-R2 between ABT-510 and vehicle treated mice (data not shown).

Endogenous TSP-1: protein expression of endogenous TSP-1 was not significantly different in any of the muscles analyzed (SOL, PLT, GA) between the treated groups (data not shown). 
CD36 levels: There was no difference in the receptor levels of CD36 in the GA as assessed by western blot (data not shown).

\author{
$\underline{\text { Apoptosis }}$ \\ Apoptosis was assessed using TUNEL staining for fragmented DNA. There was no \\ difference in the number of TUNEL positive nuclei in any of the muscles between the two \\ treated groups (Figure 5).
}

\title{
Discussion
}

The main finding of this study is that chronic exposure to the TSP-1 mimetic ABT-510 significantly decreases skeletal muscle capillarity (Figure 1). To our knowledge, these are the first data to show that chronic stimulation of the TSP-1/CD36 pathway in healthy mammals results in decreased skeletal muscle capillarity. These data are consistent with the antiangiogenic function of TSP-1 and, more importantly, support the notion that TSP-1 through its CD36 receptor is a critical regulator of skeletal muscle capillarity under physiologic conditions. Indeed, when coupled with the previous observation that loss of TSP-1 increases skeletal muscle capillarity (25) these data provide evidence that the actions of TSP-1 directly influence homeostatic maintenance and/or development of skeletal muscle microvessels.

Skeletal muscle capillarity is decreased in mimetic treated mice.

TSP-1 has been shown previously to be important in maintaining skeletal muscle capillarity, where TSP-1 KO mice have approximately 2 fold the number of capillaries as WT controls [33]. Further, TSP-1 has been shown to be important in maintaining capillarity with 
hindlimb unloading [34]. In this manuscript we build upon this small body of evidence for the importance of TSP-1 in the context of the basal regulation of angiogenesis.

The actions of TSP-1 are complex and multifunctional, due in large part to the diversity of receptors it binds, such as lipoprotein receptor-related protein 1 (LRP-1), CD47, and CD36 [11,35]. Our use of the TSP-1 mimetic ABT-510, which is a potent CD36 binding peptide, resulted in a decrease in capillarity across three different distinct skeletal muscles. This suggests that TSP-1s anti-angiogenic properties in skeletal muscle involve a CD36 mediated mechanism. Indeed, this is consistent with the physiological activity of CD36 where TSP-1 binding to CD36 has been shown to be anti-proliferative, anti-angiogenic, and pro-apoptotic [12]. The importance of the TSP1/CD36 pathway has been proven in tumorigenesis, where down regulation of TSP-1 and its binding to CD36 results in a pro-tumorigenesis environment and increase in tumor size $[14,36,37,38]$. Here we show similar actions in skeletal muscle under physiological conditions. This suggests a putative role for TSP-1 in decreasing vascularity across multiple tissues, and not just in tumors.

Given these findings, it is tempting to speculate that elevated skeletal muscle TSP-1 may serve as a biomarker for skeletal muscle dysfunction associated with several chronic conditions known to result in muscle capillary rarefaction, such as that found in diabetes $[39,40]$, and chronic heart and lung disease [41]. It may even be that therapeutic interventions to limit basal TSP-1 expression and/or reduce circulating levels of TSP-1 could be clinically exploited to attenuate the decrements in skeletal muscle function often accompanying these diseases.

Chronic stimulation of the TSP1 / CD36 pathway decreases VEGF. 
It has been shown previously that TSP-1 can counter the effects of VEGF by multiple mechanisms; including interacting directly with VEGF protein and disrupting its actions at the receptor level $[42,43,44,45,46]$. For example, TSP-1 has been suggested to bind VEGF via its type I repeat region (or 3TSR). Once bound the protein heterodimer is internalized by the scavenger receptor low density lipoprotein receptor-related protein 1 (LRP-1). Here, we show that a chronic administration of ABT-510 (a TSP-1 type I repeat mimetic), results in a decrease in total VEGF protein in both the gastrocnemius muscle and the soleus muscle, along with the decrease in capillarity. It is unclear why a similar decrease in VEGF protein was not seen in the PLT which also showed decreased capillarity, nevertheless these data support previous studies suggesting that TSP-1 may be binding and sequestering VEGF - resulting eventually in internalization and biological inactivation $[42,43,44,45,46]$. Explanations for the reduction in VEGF may also involve suppression of VEGF production and/or VEGF signaling. For example, there is a growing body of evidence showing that the internalization of the VEGF/VEGFR-2 complex by endocytosis results in downstream activation of several different signaling pathways [47]. There is also evidence that both CD36 and CD47 can associate with VEGFR-2, and when TSP-1 is present this association can prevent the VEGF ligand binding to its receptor, as well as inducing receptor dephosphorylation and preventing dimerization $[13,21,48,49]$. In this way, TSP-1 could prevent activation and/or endocytosis of the VEGF receptor, blocking yet another biologically active arm of VEGF/VEGF-R complex. Regardless of the mechanism, our data supports the notion that TSP-1 likely plays a regulatory role involving VEGF, thereby enhancing its already potent inherent anti-angiogenic capacity.

In endothelial cells, TSP-1 can also disrupt VEGF actions at the receptor level by dephosphorylating the important receptor VEGFR-2 (Flk-1) [50]. TSP-1 can disrupt VEGFR-2 
activation by binding the CLESH region of CD36, triggering a cascade of events which results in direct binding of VEGFR-2, and a resultant decrease in tyrosine phosphorylation of VEGFR-2 $[12,13,21]$. This includes dephosphorylating the important tyrosine 1175 region; which is critical to initiate downstream VEGF signaling. While it has also been shown previously that ABT-510 significantly decreases the amount and phosphorylation status of VEGFR-2 in endothelial cells in vitro [24,51], in vivo we found no significant change in the phosphorylation levels of tyrosine 1175 of VEGFR-2. Given that the mimetic is a type I repeat, and that total VEGF protein levels were decreased, we were surprised that VEGFR-2 phosphorylation was also not decreased. But, because we only have one time point, it could be that levels of phosphorylation were lower earlier in the chronic dosage but by the end of the study returned to normal levels. While ABT-510 has been shown to decrease VEGFR-2 phosphorylation in tumors [24], it may be that this response is different in skeletal muscle under physiological conditions. It could also be that phosphorylation status in vivo is not lowered by endogenous TSP-1 (or the addition of a mimetic), rather TSP-1 limits or prevents an increase in phosphorylation levels with a VEGF stimulus. This is yet to be elucidated.

Administration of ABT-510 did not significantly alter endogenous levels of TSP-1 in skeletal muscle, indicating that there was no feedback response from the mimetic binding to CD36 to alter endogenous TSP-1 levels in skeletal muscle. However, the reduction of skeletal muscle VEGF protein levels in association with the mimetic adds support to the idea that physiologic angiogenesis is controlled by a balance between both positive and negative angiogenic factors, particularly VEGF and TSP-1. It would have been interesting to deliver a higher dose of ABT-510 (> $30 \mathrm{ug} / \mathrm{kg} / \mathrm{day}$ ) which may have resulted in greater reductions in muscle VEGF and capillarity; however the dose used was the maximum we could deliver using 
the small pumps suitable for mice and the 2-week duration we sought. Although shorter duration would allow greater concentrations of drug delivery, we opted to deliver the drug for 14 days to ensure that vascular remodeling would have sufficient time to occur. It could be hypothesized that tipping the scale towards an anti-angiogenic state (stimulated by the presence of ABT-510) would result in a response of positive angiogenic factors to counter this challenge. However, at least with respect to VEGF this was not the case. A decrease in VEGF and no change in endogenous TSP-1 suggests that the muscle has been pushed towards an antiangiogenic phenotype.

Regardless of mechanism, these data show that the altered angiogenic state of the skeletal muscle can be driven by an increase in a TSP-1 mimetic. The resulting decrease in capillarity establishes the importance of TSP-1/ CD36 pathway in maintaining and regulating basal skeletal muscle microvessel density.

Skeletal muscle and endothelial apoptotic levels do not differ between groups.

In addition to its anti-angiogenic properties, TSP-1 is also been known to promote apoptosis $[6,52,53,54,55,56,57,58]$. TSP-1 is known to induce apoptosis through the CD36 pathway, as shown in previous studies, including those using ABT-510 [5,24,59]. Endothelial apoptosis is a natural mechanism by which capillary regression occurs $[60,61,62,63]$, and has been shown to be central in muscle atrophy during some pathologies, aging, and disuse $[64,65,66,67,68]$. Knowing this, we performed TUNEL staining to look at end stage apoptosis (DNA fragmentation) in all three of the excised muscles. Surprisingly, we found no evidence of apoptosis in any of the muscles, either myocyte or endothelial. The reason for this remains unclear. This is, however, the first in vivo report of ABT-510s affects on skeletal muscle and it 
could be that ABT-510 is not sufficient to induce myocyte apoptosis in skeletal muscle under these conditions. Indeed, much of the work with TSP-1 and apoptosis pertains to cancer models (e.g. tumorigenesis), endothelial cells, and is typically done in vitro. It is possible that TSP-1 is not as potent a pro-apoptotic protein in skeletal muscle as that seen in endothelial cells or tumors. This is supported by the finding that there was no change in muscle fiber cross-sectional area (Figures 2 and 3). Whether or not the ABT-510, or the TSP-1/CD36 axis, alters in vivo skeletal muscle myocyte apoptosis will require further study.

However, this does not explain why we saw no endothelial cell apoptosis. As mentioned previously, our study only examines one time point. It is possible that apoptosis occurred at an earlier time point than our assessment at the 14 day time point. Indeed, it has been shown previously that hindlimb unloading results in capillary loss in as little as 5 days [34]. If changes in endothelial cell number, and therefore total vasculature, are occurring by 5-7 days it may not be surprising that evidence of apoptosis has been lost by the end of the study (14 days). That is, perhaps apoptosis has already occurred, the cells cleared from the tissue, and the architecture of the vascular network at 14 days has already returned to a new steady state condition absent of apoptotic nuclei. We have yet to elucidate this and more experimentation is needed to determine exactly when apoptosis and regression is occurring.

\section{Summary}

In conclusion, we provide evidence for the importance of TSP-1/CD36 pathway in regulating basal skeletal muscle capillarity by showing that a chronic dosage of a TSP-1 mimetic for the CD36 pathway decreases skeletal muscle capillarity and VEGF expression. We did not find any differences in the VEGFR-2 expression or its phosphorylation status, nor was there 
greater skeletal muscle apoptosis. These data show that, despite the multifunctional effects of TSP-1 and its CD36 receptor, the primary consequence of elevating circulating TSP-1 (i.e. using a TSP-1 mimetic targeted to its CD36 receptor) relates to its anti-angiogenic function. These data may be useful in exploring therapeutic interventions for individuals with skeletal muscle dysfunction resulting from capillary regression. 


\section{Acknowledgements}

The authors wish to thank Kathleen Roberts, W. Kyle Mandler, Sara Olenich, and Dharendrah Thapa for their assistance in the laboratory in preparing pumps and processing the tissues. We would also like to thank Dr. Josef Anrather of The Weill Cornell Medical College for his help and advice on CD36 analysis. 


\section{Tables}

Table 1: Muscle and Body Masses

\begin{tabular}{|l|c|c|}
\hline \hline & Vehicle & ABT-510 \\
\hline Body Mass (g) & $27.5 \pm 1.23$ & $27.1 \pm 0.87$ \\
\hline GA (mg) & $136.8 \pm 4.56$ & $140.8 \pm 4.09$ \\
\hline PLT (mg) & $19.7 \pm 0.90$ & $19.0 \pm 0.53$ \\
\hline SOL (mg) & $7.7 \pm 0.47$ & $7.8 \pm .048$ \\
\hline HRT (mg) & $123.5 \pm 5.76$ & $134.3 \pm 5.82$ \\
\hline GA/BM (mg/g) & $5.0 \pm 0.07$ & $5.2 \pm 0.12$ \\
\hline PLT/BM (mg/g) & $0.72 \pm 0.04$ & $0.70 \pm 0.02$ \\
\hline SOL/BM (mg/g) & $0.29 \pm 0.01$ & $0.28 \pm 0.01$ \\
\hline HRT/BM (mg/g) & $4.4 \pm 0.26$ & $5.0 \pm 0.17$ \\
\hline
\end{tabular}

Values: Mean \pm Standard Error. $* \mathrm{P}=0.05$ 
Citations

1. Breen EC, Johnson EC, Wagner H, Tseng HM, Sung LA, et al. (1996) Angiogenic growth factor mRNA responses in muscle to a single bout of exercise. Journal of Applied Physiology 81: 355-361.

2. Olfert IM, Howlett RA, Tang K, Dalton ND, Gu Y, et al. (2009) Muscle-specific VEGF deficiency greatly reduces exercise endurance in mice. The Journal of Physiology 587: 1755.

3. Roudier E, Gineste C, Wazna A, Dehghan K, Desplanches D, et al. (2010) Angio-adaptation in unloaded skeletal muscle: new insights into an early and muscle type-specific dynamic process. The Journal of Physiology 588: 4579-4591.

4. Olfert IM, Birot O (2011) Importance of Anti-angiogenic Factors in the Regulation of Skeletal Muscle Angiogenesis. Microcirculation 18: 316-330.

5. Jack L (2000) The functions of thrombospondin-1 and-2. Current Opinion in Cell Biology 12: 634-640.

6. Jimenez B, Volpert OV, Crawford SE, Febbraio M, Silverstein RL, et al. (2000) Signals leading to apoptosis-dependent inhibition of neovascularization by thrombospondin-1. Nature medicine 6: 41-48.

7. Lange-Asschenfeldt B, Weninger W, Velasco P, Kyriakides TR, von Andrian UH, et al. (2002) Increased and prolonged inflammation and angiogenesis in delayed-type hypersensitivity reactions elicited in the skin of thrombospondin-2-deficient mice. Blood 99: 538-545.

8. Varma V, Yao-Borengasser A, Bodles AM, Rasouli N, Phanavanh B, et al. (2008) Thrombospondin-1 Is an Adipokine Associated With Obesity, Adipose Inflammation, and Insulin Resistance. Diabetes 57: 432-439.

9. Ridnour LA, Isenberg JS, Espey MG, Thomas DD, Roberts DD, et al. (2005) Nitric oxide regulates angiogenesis through a functional switch involving thrombospondin-1. Proceedings of the National Academy of Sciences of the United States of America 102: 13147-13152.

10. Kvansakul M, Adams JC, Hohenester E (2004) Structure of a thrombospondin C-terminal fragment reveals a novel calcium core in the type 3 repeats. EMBO J 23: 1223-1233.

11. Chen H, Herndon ME, Lawler J (2000) The cell biology of thrombospondin-1. Matrix Biology 19: 597-614.

12. Febbraio M, Hajjar DP, Silverstein RL (2001) CD36: a class B scavenger receptor involved in angiogenesis, atherosclerosis, inflammation, and lipid metabolism. Journal of Clinical Investigation 108: 785-792.

13. Primo L, Ferrandi C, Roca C, Marchiò S, di Blasio L, et al. (2005) Identification of CD36 molecular features required for its in vitro angiostatic activity. The FASEB journal 19: 1713-1715.

14. Ren B, Yee KO, Lawler J, Khosravi-Far R (2006) Regulation of tumor angiogenesis by thrombospondin-1. Biochimica et Biophysica Acta (BBA) - Reviews on Cancer 1765: 178-188.

15. Hoekstra R, de Vos FYFL, Eskens FALM, Gietema JA, van der Gaast A, et al. (2005) Phase I Safety, Pharmacokinetic, and Pharmacodynamic Study of the Thrombospondin1- Mimetic Angiogenesis Inhibitor ABT-510 in Patients With Advanced Cancer. Journal of Clinical Oncology 23: 5188-5197. 
16. Reiher FK, Volpert OV, Jimenez B, Crawford SE, Dinney CP, et al. (2002) Inhibition of tumor growth by systemic treatment with thrombospondin-1 peptide mimetics. International Journal of Cancer 98: 682-689.

17. Rusk A, McKeegan E, Haviv F, Majest S, Henkin J, et al. (2006) Preclinical Evaluation of Antiangiogenic Thrombospondin-1 Peptide Mimetics, ABT-526 and ABT-510, in Companion Dogs with Naturally Occurring Cancers. Clinical Cancer Research 12: 74447455.

18. Olfert IM, Breen EC, Gavin TP, Wagner PD (2006) Temporal thrombospondin-1 mRNA response in skeletal muscle exposed to acute and chronic exercise. Growth Factors 24: 253-259.

19. Olfert IM (2012) Unpublished Data.

20. Frazier EP, Isenberg JS, Shiva S, Zhao L, Schlesinger P, et al. (2011) Age-dependent regulation of skeletal muscle mitochondria by the thrombospondin-1 receptor CD47. Matrix Biology.

21. Dawson DW, Pearce SFA, Zhong R, Silverstein RL, Frazier WA, et al. (1997) CD36 mediates the in vitro inhibitory effects of thrombospondin-1 on endothelial cells. The Journal of cell biology 138: 707.

22. Haviv F, Bradley MF, Kalvin DM, Schneider AJ, Davidson DJ, et al. (2005) Thrombospondin-1 mimetic peptide inhibitors of angiogenesis and tumor growth: design, synthesis, and optimization of pharmacokinetics and biological activities. Journal of medicinal chemistry 48: 2838-2846.

23. Anderson JC, Grammer JR, Wang W, Nabors LB, Henkin J, et al. (2007) ABT-510, a modified type 1 repeat peptide of thrombospondin, inhibits malignant glioma growth in vivo by inhibiting angiogenesis. Cancer biology \& therapy 6: 454.

24. Greenaway J, Henkin J, Lawler J, Moorehead R, Petrik J (2009) ABT-510 induces tumor cell apoptosis and inhibits ovarian tumor growth in an orthotopic, syngeneic model of epithelial ovarian cancer. Molecular cancer therapeutics 8: 64.

25. Rusk A, McKeegan E, Haviv F, Majest S, Henkin J, et al. (2006) Preclinical evaluation of antiangiogenic thrombospondin-1 peptide mimetics, ABT-526 and ABT-510, in companion dogs with naturally occurring cancers. Clinical cancer research 12: 7444.

26. Yap R, Veliceasa D, Emmenegger U, Kerbel RS, McKay LM, et al. (2005) Metronomic lowdose chemotherapy boosts CD95-dependent antiangiogenic effect of the thrombospondin peptide ABT-510: a complementation antiangiogenic strategy. Clinical cancer research 11: 6678 .

27. Campbell NE, Greenaway J, Henkin J, Moorehead RA, Petrik J The Thrombospondin-1 Mimetic ABT-510 Increases the Uptake and Effectiveness of Cisplatin and Paclitaxel in a Mouse Model of Epithelial Ovarian Cancer. Neoplasia Press Inc.

28. Isenberg JS, Yu C, Roberts DD (2008) Differential effects of ABT-510 and a CD36-binding peptide derived from the type 1 repeats of thrombospondin- 1 on fatty acid uptake, nitric oxide signaling, and caspase activation in vascular cells. Biochemical Pharmacology 75: 875-882.

29. Hasina R, Martin LE, Kasza K, Jones CL, Jalil A, et al. (2009) ABT-510 is an effective chemopreventive agent in the mouse 4-nitroquinoline 1-oxide model of oral carcinogenesis. Cancer Prevention Research 2: 385. 
30. Joyce D, Mulji G, Gutierrez L, Castellino F (2006) Evaluation of the Thrombospondin-1 analogue ABT-510 in the APCMin/+ mouse intestinal adenoma model. Journal of Clinical Oncology 24: 13545.

31. Mrázková O, Grim M, Carlson BM (1986) Enzymatic heterogeneity of the capillary bed of rat skeletal muscles. American Journal of Anatomy 177: 141-148.

32. Lojda Z (1979) Studies on dipeptidyl(amino)peptidase IV (glycyl-proline naphthylamidase). Histochemistry 59: 153-166.

33. Malek MH, Olfert IM (2009) Global deletion of thrombospondin 1 increases cardiac and skeletal muscle capillarity and exercise capacity in mice. Experimental physiology 94 : 749-760.

34. Roudier E, Gineste C, Wazna A, Dehghan K, Desplanches D, et al. (2010) Angio-adaptation in unloaded skeletal muscle: new insights into an early and muscle type-specific dynamic process. The Journal of Physiology 588: 4579.

35. Bornstein P (2009) Thrombospondins function as regulators of angiogenesis. Journal of Cell Communication and Signaling 3: 189-200.

36. Lawler J (2000) The functions of thrombospondin-1 and-2. Current opinion in cell biology 12: 634-640.

37. Zhang X, Lawler J (2007) Thrombospondin-based antiangiogenic therapy. Microvascular research 74: 90-99.

38. Lawler J, Detmar M (2004) Tumor progression: the effects of thrombospondin-1 and -2 . The International Journal of Biochemistry \&amp; Cell Biology 36: 1038-1045.

39. Kivela R, Silvennoinen M, Lehti M, Jalava S, Vihko V, et al. (2008) Exercise-induced expression of angiogenic growth factors in skeletal muscle and in capillaries of healthy and diabetic mice. Cardiovascular Diabetology 7: 13.

40. Kivelä R, Silvennoinen M, Touvra A-M, Lehti TM, Kainulainen H, et al. (2006) Effects of experimental type 1 diabetes and exercise training on angiogenic gene expression and capillarization in skeletal muscle. The FASEB journal 20: 1570-1572.

41. Duscha BD, Kraus WE, Keteyian SJ, Sullivan MJ, Green HJ, et al. (1999) Capillary density of skeletal muscle: A contributing mechanism for exercise intolerance in class II-III chronic heart failure independent of other peripheral alterations. Journal of the American College of Cardiology 33: 1956-1963.

42. Greenaway J, Lawler J, Moorehead R, Bornstein P, Lamarre J, et al. (2007) Thrombospondin-1 inhibits VEGF levels in the ovary directly by binding and internalization via the low density lipoprotein receptor-related protein-1 (LRP-1). Journal of cellular physiology 210: 807-818.

43. Gupta K, Gupta P, Wild R, Ramakrishnan S, Hebbel R (1999) Binding and displacement of vascular endothelial growth factor (VEGF) by thrombospondin: Effect on human microvascular endothelial cell proliferation and angiogenesis. Angiogenesis 3: 147-158.

44. Iruela-Arispe ML, Lombardo M, Krutzsch HC, Lawler J, Roberts DD (1999) Inhibition of Angiogenesis by Thrombospondin-1 Is Mediated by 2 Independent Regions Within the Type 1 Repeats. Circulation 100: 1423-1431.

45. Rodríguez-Manzaneque JC, Lane TF, Ortega MA, Hynes RO, Lawler J, et al. (2001) Thrombospondin-1 suppresses spontaneous tumor growth and inhibits activation of matrix metalloproteinase-9 and mobilization of vascular endothelial growth factor. Proceedings of the National Academy of Sciences of the United States of America 98: 12485. 
46. Sun J, Hopkins BD, Tsujikawa K, Perruzzi C, Adini I, et al. (2009) Thrombospondin-1 modulates VEGF-A-mediated Akt signaling and capillary survival in the developing retina. American Journal of Physiology-Heart and Circulatory Physiology 296: H1344.

47. Simons M (2012) An Inside View: VEGF Receptor Trafficking and Signaling. Physiology 27: 213-222.

48. Kaur S, Martin-Manso G, Pendrak ML, Garfield SH, Isenberg JS, et al. (2010) Thrombospondin-1 Inhibits VEGF Receptor-2 Signaling by Disrupting Its Association with CD47. Journal of Biological Chemistry 285: 38923-38932.

49. Zhang X, Kazerounian S, Duquette M, Perruzzi C, Nagy JA, et al. (2009) Thrombospondin-1 modulates vascular endothelial growth factor activity at the receptor level. The FASEB journal 23: 3368-3376.

50. Zhang X, Kazerounian S, Duquette M, Perruzzi C, Nagy JA, et al. (2009) Thrombospondin-1 modulates vascular endothelial growth factor activity at the receptor level. The FASEB journal 23: 3368.

51. Reiher FK, Volpert OV, Jimenez B, Crawford SE, Dinney CP, et al. (2002) Inhibition of tumor growth by systemic treatment with thrombospondin 1 peptide mimetics. International journal of cancer 98: 682-689.

52. Friedl P, Vischer P, Freyberg M (2002) The role of thrombospondin-1 in apoptosis. Cellular and molecular life sciences 59: 1347-1357.

53. Guo N, Krutzsch HC, Inman JK, Roberts DD (1997) Thrombospondin 1 and type I repeat peptides of thrombospondin 1 specifically induce apoptosis of endothelial cells. Cancer research 57: 1735.

54. Miao W-M, Lin Seng W, Duquette M, Lawler P, Laus C, et al. (2001) Thrombospondin-1 Type 1 Repeat Recombinant Proteins Inhibit Tumor Growth through Transforming Growth Factor- $\beta$-dependent and -independent Mechanisms. Cancer research 61: 78307839.

55. Nör J, Mitra RS, Sutorik MM, Mooney DJ, Castle VP, et al. (2000) Thrombospondin-1 induces endothelial cell apoptosis and inhibits angiogenesis by activating the caspase death pathway. Journal of vascular research 37: 209-218.

56. Isenberg J, Frazier W, Roberts D (2008) Thrombospondins: from structure to therapeutics: Thrombospondin-1: a physiological regulator of nitric oxide signaling. Cellular and Molecular Life Sciences CMLS 65: 728-742.

57. Isenberg JS, Hyodo F, Matsumoto KI, Romeo MJ, Abu-Asab M, et al. (2007) Thrombospondin-1 limits ischemic tissue survival by inhibiting nitric oxide-mediated vascular smooth muscle relaxation. Blood 109: 1945.

58. Isenberg JS, Ridnour LA, Perruccio EM, Espey MG, Wink DA, et al. (2005) Thrombospondin-1 inhibits endothelial cell responses to nitric oxide in a cGMPdependent manner. Proceedings of the National Academy of Sciences of the United States of America 102: 13141.

59. Wintergerst ES, Jelk J, Rahner C, Asmis R (2000) Apoptosis induced by oxidized low density lipoprotein in human monocyte-derived macrophages involves CD36 and activation of caspase-3. European Journal of Biochemistry 267: 6050-6059.

60. Meeson AP, Argilla M, Ko K, Witte L, Lang RA (1999) VEGF deprivation-induced apoptosis is a component of programmed capillary regression. Development 126: 14071415. 
61. Tang K, Breen EC, Gerber H-P, Ferrara NMA, Wagner PD (2004) Capillary regression in vascular endothelial growth factor-deficient skeletal muscle. Physiological Genomics 18: 63-69.

62. Meeson A, Palmer M, Calfon M, Lang R (1996) A relationship between apoptosis and flow during programmed capillary regression is revealed by vital analysis. Development 122 : 3929-3938.

63. Dimmeler S, Zeiher AM (2000) Endothelial Cell Apoptosis in Angiogenesis and Vessel Regression. Circulation research 87: 434-439.

64. Degens H, Alway SE (2006) Control of muscle size during disuse, disease, and aging. International journal of sports medicine 27: 94-99.

65. Siu PM, Pistilli EE, Butler DC, Alway SE (2005) Aging influences cellular and molecular responses of apoptosis to skeletal muscle unloading. American Journal of Physiology Cell Physiology 288: C338-C349.

66. dalla Libera L, Zennaro R, Sandri M, Ambrosio GB, Vescovo G (1999) Apoptosis and atrophy in rat slow skeletal muscles in chronic heart failure. American Journal of Physiology - Cell Physiology 277: C982-C986.

67. Dupont-Versteegden EE (2005) Apoptosis in muscle atrophy: Relevance to sarcopenia. Experimental Gerontology 40: 473-481.

68. Allen DL, Linderman JK, Roy RR, Bigbee AJ, Grindeland RE, et al. (1997) Apoptosis: a mechanism contributing to remodeling of skeletal muscle in response to hindlimb unweighting. American Journal of Physiology - Cell Physiology 273: C579-C587. 


\section{Figure Legends}

Figure 1: Representative figures of the histology sections. A) Superficial Gastrocnemius Muscle B) Deep Gastrocnemius Muscle C) Plantaris Muscle, D) Soleus Muscle.

Figure 2: $\underline{\text { Superficial gastrocnemius capillarity, but not deep, is decreased in the ABT-510 }}$ group.

Chronic administration of ABT-510 30mg/kg/day decreased capillary to fiber ratio (C:F) and capillary density (CD) in the superficial gastrocnemius (GA). Despite a similar trend, no significant changes in capillarity were seen in the deep GA. There was no change in fiber cross sectional area in either the deep or superficial portions of the GA. $*=\mathrm{P} \leq 0.05$

Figure 3: Plantaris and soleus capillarity is decreased in the ABT-510 group. Chronic administration of ABT-510 30mg/kg/day decreased capillary to fiber ratio (C:F) in the plantaris muscle (PLT) but did not decrease capillary density (CD). ABT-510 decreased capillary to fiber ratio $(\mathrm{C}: \mathrm{F})$ and capillary density $(\mathrm{CD})$ in the soleus (SOL). There was no change in fiber cross sectional area in either the PLT or SOL muscle. $*=\mathrm{P} \leq 0.05$

Figure 4: $\underline{\text { Skeletal muscle VEGF protein content is decreased in the ABT-510 group, but not }}$ $\underline{\text { serum levels. }}$

Chronic administration of the mimetic (ABT-510 30mg/kg/day) decreased VEGF protein in the GA, and SOL muscles of C57/BLK6 mice. There was no change in the PLT muscle or in Serum levels. $*=\mathrm{P} \leq 0.05$ 
Figure 5: There is no difference in skeletal muscle apoptosis between the mimetic and control groups

TUNEL staining shows that chronic administration of the mimetic (ABT-510 30mg/kg/day) did not increase or decrease skeletal muscle apoptosis in any of the muscles tested. Representative GA samples, PLT and SOL data not shown. A) Representative mimetic GA B) Representative positive control mimetic GA C) Representative control GA D) Representative positive control vehicle GA. 


\section{Chapter 4: TSP-1 disrupts mitochondrial enzyme activity through the CD36 receptor}




\section{TSP-1 disrupts mitochondrial enzyme activity through the CD36 receptor}

Gerald N. Audet, Emidio Pistilli, Dharendra Thapa, Janelle Stricker, John M. Hollander, I. Mark Olfert

West Virginia University School of Medicine, Center for Cardiovascular and Respiratory

Sciences, Division of Exercise Physiology, Morgantown, WV, USA

Short Title: TSP-1 alters oxidative enzyme activity

Address for correspondence:

Mark Olfert, PhD

West Virginia University School of Medicine

Center for Cardiovascular and Respiratory Sciences

Division of Exercise Physiology

PO Box 9105

Morgantown, WV 26508

Phone 304-293-7597

molfert@hsc.wvu.edu

This paper has been formatted for submission to the Acta Physiologica 


\section{Abstract (195 words):}

Thrombospondin-1 (TSP-1) is critical angiogenic mediator in skeletal muscle and recently has been shown to be a regulator of mitochondrial biogenesis, however its role in controlling mitochondrial function remains unknown. In this study we hypothesized that the TSP-1 mimetic (ABT-510) would decrease mitochondrial electron transport enzyme (mETC) activity and decrease skeletal muscle function, while an opposite effect was expected for whole-body TSP-1 KO mice. We studied 3 month old WT and TSP-1 KO mice. WT mice were given the TSP-1 mimetic systemically using osmotic mini pumps for 14 days. We measured both kinetic and fatigue characteristics of the soleus (SOL) and extensor digitorum longus (EDL) muscles of all groups. We found that mimetic treated mice had $29 \%$ and $42 \%$ decrease in mETC complex III and IV activity, respectively, with a similar (albeit not statistically significant) $20 \%$ decreased complex I activity $(\mathrm{p}<0.07)$. TSP-1 KO mice showed an opposite trend for an increase in complex IV ( $\mathrm{p}<0.07)$. Surprisingly, we saw no change in either muscle kinetic or fatigue characteristics in either the SOL or EDL muscle. This work suggests, in addition to its antiangiogenic effects, TSP-1 can also influence mitochondrial respiration activity through its CD36 receptor pathway. 


\section{Introduction}

Thrombospondin-1 (TSP-1) is a large 450kD extracellular protein that has a wide array of functions. First discovered for its role in wound healing and platelet activation, TSP-1 has also been shown to influence inflammation, nitric oxide signaling, and matrix remodeling [1-5]. Acting through the CD36 receptor, TSP-1 has proven to prevent endothelial cell adhesion, growth, and migration as well as potently regulate in vivo capillarity in a variety of models [610]. The anti-angiogenic effects of TSP-1 in cancer pathology are well established, and the evidence continues to mount for its pivotal role in skeletal muscle [9-12].

TSP-1 has also recently been shown to decrease mitochondrial biogenesis in young animals [13]. The mechanism by which TSP-1 controls mitochondrial biogenesis and density is poorly understood, but may be linked to nitric oxide signaling [4, 13-16]. Another mechanism may also involve TSP-1s inhibition of angiogenesis, particularly in skeletal muscle $[9,10,17]$. Indeed, reduced skeletal muscle capillarity could limit the supply of oxygen and substrate necessary for mitochondria to produce ATP essential for contractile function and induce a reduction in mitochondrial density $[18,19]$.

Acting through the CD36 receptor TSP-1 has also been shown to promote apoptosis, [1, 20-24], however the role of TSP-1 and CD36 in myocyte and endothelial cell apoptosis in vivo is not well characterized. We have shown previously that a 14 day chronic exposure to the TSP-1 mimetic ABT-510 did not result in apoptosis in skeletal muscle [9]. ABT-510 is a mimetic of the conserved type I repeat region of TSP-1 [25] that has been shown previously to inhibit angiogenesis through the CD36 pathway $[7,8,26]$ and prevent vascular growth through its proapoptotic actions [27-33]. Nevertheless, we found no signs of apoptosis in skeletal muscle following 14 days of treatment with ABT-510, leading to the speculation that apoptosis might 
have occurred at an earlier time point with this treatment. If true, apoptotic cells could have been removed and the tissue could have been devoid of DNA fragmentation or apoptotic tissue at the 14-day time point. Given that apoptosis is the most likely mechanism by which capillaries losses are expected [34-37], we hypothesized that skeletal muscle apoptosis would be evident in mice with 3 and/or 7 days treatment with ABT-510.

Given that we have previously shown that a TSP-1 mimetic decreases capillarity, whereas whole body loss of TSP-1 increases skeletal muscle capillarity and improves whole body exercise $[9,10]$, we evaluated the effect of a CD36-mediated TSP-1 mimetic on skeletal muscle mitochondrial function. We hypothesized that TSP-1 activation of the CD36 pathway could significantly influence mitochondrial activity and therefore increase mitochondrial electron transport chain (ETC) enzyme activity in the TSP-1 KO animals, while a decrease in ETC activity and muscle function would occur in mice subjected to a chronic treatment with the TSP1 mimetic ABT-510.

\section{Materials and Methods:}

\section{$\underline{\text { Animals }}$}

This study used 10-12 week old male C57BL/6 (WT) and TSP-1 knockout (KO) mice purchased from Jackson Laboratories (Strain no. 000664 and 006141, respectively, Bar Harbor, ME). Four groups of WT mice were subject to either 3 or 7 days of mimetic (3-ABT or 7-ABT) or vehicle control (3-CON or 7-CON) (3 Day: N=4/group; 7 Day: N=10/group). Another group of WT mice were given a 14 day chronic dose of the mimetic (14-ABT) or vehicle control (14$\mathrm{CON}$ ) (N=8/group). The final group consisted of untreated TSP-1 KO and WT mice (N=7- 
8/group). All procedures were approved by the West Virginia University Institutional Animal Care and Use Committee.

\section{Osmotic Pump Drug Delivery}

The TSP-1 mimetic ABT-510 was generously provided by Abbott (Abbott, IL). This compound was chosen for use in this study based on previous work showing its effectiveness in decreasing capillarity in tumors, both in vitro and in vivo [25, 27-30, 38, 39]. ABT-510 was dissolved in $5 \%$ dextrose (vehicle) over a 48 hour period at 4 degrees to ensure the compound was fully dissolved, and then inserted into mini-osmotic pumps (Model 1002, Alzet Osmotic Pumps, Cupertino, CA). Pumps were filled with either the drug or vehicle solution 24 hours in advance of implantation as per manufacture instructions. We delivered the maximum concentration of $30 \mathrm{mg} / \mathrm{kg} /$ day based on the maximum pump capacity $(100 \mu \mathrm{L})$, the mass of the animals, and the solubility of the drug (ABT-510 solubility curves [25]). Pumps were surgically inserted (flow-moderator first) subcutaneously in the scapular region while the mice were under anesthesia ( $2 \%$ isoflurane). The surgical site was disinfected with iodine and closed using sutures. Animals were then housed individually and observed daily for the remainder of the study for pain or distress. Pumps remained in the animals for either 3 days (3-CON, 3-ABT), 7 days (7-CON, 7-ABT) or 14 days, (14-CON, 14-ABT) depending on the randomly assigned treatment group, after which they were sacrificed and tissue/organ samples were collected and stored at $-80^{\circ}$ for analysis.

\section{$\underline{E x}$ vivo muscle function}

Muscle contractile properties were examined in the extensor digitorum longus (EDL) and soleus (SOL) muscles. Parameters analyzed from isometric contractions included peak isometric twitch force, time to peak twitch tension, $1 / 2$ relaxation time of twitch contraction, and peak 
isometric tetanic force, as previously described [40]. Isometric contractions were stimulated in muscles ex vivo using a bi-phase stimulator at a stimulation frequency of $120 \mathrm{~Hz}$ for EDL muscles and $80 \mathrm{~Hz}$ for soleus muscles, a stimulation current of 28 Volts and lasting $500 \mathrm{~ms}$. Muscle fatigue was analyzed using a repeated stimulation protocol lasting 6 minutes and consisting of repeated $40 \mathrm{~Hz}$ tetanic trains that occurred once every second and lasted $330 \mathrm{~ms}$ [41]. Muscle length was adjusted to obtain the maximal twitch response (i.e. $\mathrm{L}_{0}$ ). Muscles were stimulated in an oxygenated ringers solution composed of: $100 \mathrm{mM} \mathrm{NaCl}, 4.7 \mathrm{mM} \mathrm{KCl}, 3.4 \mathrm{mM}$ $\mathrm{CaCl}_{2}, 1.2 \mathrm{mM} \mathrm{KH}_{2} \mathrm{PO}_{4}, 1.2 \mathrm{mM} \mathrm{MgSO}_{4}, 25 \mathrm{mM}$ HEPES, and 5.5mM D-glucose. Three twitch contractions and three tetanic contractions were performed all separated by 2 minutes. Following isometric contractions, the muscles remained in oxygenated ringers for 5 minutes prior to the repeated stimulation fatigue protocol. The fatigue index was calculated as the difference in force from the first contraction and every $10^{\text {th }}$ subsequent contraction, and plotted as a fatigue curve. Muscles were flash frozen in isopentane cooled to the temperature of liquid nitrogen and stored at $-80^{\circ} \mathrm{C}$. Muscle fiber cross sectional area (FCSA) was calculated by dividing the muscle mass by the product of the muscle density coefficient $\left(1.06 \mathrm{~g}^{\mathrm{cm}}{ }^{3}\right)$, muscle optimal length $\left(\mathrm{L}_{0}\right)$, and the fiber length coefficient $(\mathrm{EDL}=0.45, \mathrm{SOL}=0.69)$. This whole muscle

CSA value was used to calculate specific force (i.e. absolute force mN'muscle $\mathrm{CSA}^{-1}$ ) $[42,43]$.

\section{Cell Death}

Total cell death of skeletal muscle homogenate was analyzed from 200ug of tissue using a commercially available cell death ELISA kit (cat \# 11774425001, Roche, Indianapolis, IN, USA).

\section{$\underline{\text { Mitochondrial Assessments }}$}


ETC activities of complexes I, III, and IV were measured spectrophotometrically from whole muscle homogenate as previously described [44-46]. Briefly, complex I activity was determined by measuring the oxidation of NADH at $340 \mathrm{~nm}$ as previously described [45]. The assay mixture for complex I contained $25 \mathrm{mM}$ potassium phosphate buffer (pH 7.2), $5 \mathrm{mM}$ $\mathrm{MgCl} 2,2 \mathrm{mM} \mathrm{KCN}, 2.5 \mathrm{mg} / \mathrm{ml} \mathrm{BSA}, 50 \mu \mathrm{M}$ NADH, $10 \mu \mathrm{M}$ decylubiquinone, and $2 \mu \mathrm{g} / \mathrm{ml}$ antimycin A. The reaction was initiated by the addition of muscle homogenate, and enzyme activity was measured for $3 \mathrm{~min}$, with values recorded every $10 \mathrm{~s}$ after the initiation of the reaction. Complex I-specific activity was inhibited by $2 \mu \mathrm{g} / \mathrm{ml}$ rotenone. Complex III activity was determined as previously described $[44,45]$ by following the reduction of cytochrome $c$ at $550 \mathrm{~nm}$ in the presence of reduced decylubiquinone $(50 \mu \mathrm{M})$. Briefly, the assay buffer for complex III consisted of $500 \mathrm{mM}$ sucrose, $2 \mathrm{mM}$ EDTA, $100 \mathrm{mM}$ Tris $\cdot \mathrm{HCl},(\mathrm{pH} 7.4), 1 \mathrm{mM}$ cytochrome $c, 200 \mathrm{mM} \mathrm{KCN}, 1 \mathrm{mg} / \mathrm{ml}$ antimycin A, and educed decylubiquinone. Finally, complex IV activity was determined by measuring the oxidation of cytochrome $c$ at $550 \mathrm{~nm}$. Briefly, the assay mixture for complex IV consisted of $10 \mathrm{mM}$ phosphate buffer (pH 7.4) and 20 $\mu \mathrm{M}$ reduced cytochrome $c$. Protein content was determined as described previously via the bradford assay [47], and values are expressed as activities in $\mathrm{nmol}$ substrate consumed $\cdot \mathrm{min}^{-1} \cdot \mathrm{mg}$ $\operatorname{protein}^{-1}$.

\section{$\underline{\text { Statistics }}$}

All data are presented as means \pm SEM. To examine body mass, organ masses, muscle capillarity ex vivo muscle stimulation, and mETC activity we used a student's T-test. An alpha level at $\mathrm{P} \leq 0.05$ was selected for statistical significance. 


\section{Results}

\section{Muscle Mass}

There was no difference in muscle or body masses between the 3, 7, or 14 day ABT animals and their respective controls (Table 1). There was also no absolute difference in the TSP-1 KO muscle mass compared to WT in any of the muscles, but when normalized to for body mass GA was decreased by $21 \%$ in the TSP- $1 \mathrm{KO}$ animals compared to WT controls $(\mathrm{P}<0.01)$, and a similar trend for a $12 \%$ decrease in the PLT $(\mathrm{P}=0.051)($ Table 1$)$. The SOL was unchanged when compared to body mass. These data are similar to those previously observed for these muscles in TSP-1 KO mice [10]

\section{Cell Death}

There was no evidence of cell death as measured by cell death ELISA in the skeletal muscle of either the 3-ABT or 7-ABT groups compared to their respective CON groups (Figure 1). There was also no significant difference in cell death between 3 and 7 day time points.

\section{Mitochondrial enzyme activity}

Mice treated with ABT-510 displayed a significant $29 \%$ and $42 \%$ decrease in complex III and IV activity $(\mathrm{p}<0.05)$, and to a lesser extent a decrease in complex I activity $(\mathrm{p}<0.07)$, in the gastrocnemius muscle (Table 2). In contrast, TSP-1 KO mice tended to display an increase in gastrocnemius muscle complex IV activity $(\mathrm{p}<0.07)($ Table 2$)$. A similar downward trend was

observed in complex III activity in the SOL muscle (i.e. 50\% decrease, p<0.07) of ABT-510 treated mice, but no significance was reached for any of the complexes in that muscle (data not shown). Likewise, no difference in any of the complexes in the PLT of the ABT treated mice was seen (data not shown).

\section{Ex vivo muscle function}


There was a trend for a decrease in peak tetanus force $(31 \%, \mathrm{P}=0.06)$ and peak tetanus force/fiber cross sectional area $(30 \%, \mathrm{P}=0.12)$ in the SOL muscle of 14 day ABT treated mice compared to CON mice (Figure 2), but neither proved statistically significant. Surprisingly, no difference in fatigue profiles of either the SOL or EDL muscle were seen (Figure 3). Likewise, muscle fatigue response was not different between TSP-1 KO and WT mice (Figure 4). There was no difference in any muscle anatomical or physiological parameter measured during tetanus contractions (Tables 3 and 4)

\section{Discussion}

The main finding of this study is that mitochondrial complex III and IV activity is decreased in the gastrocnemius of mice given the TSP-1 mimetic ABT-510, while global deletion of TSP-1 resulted in a trend for higher complex IV enzyme activities, suggesting that the TSP-1/CD36 pathway can also influence mitochondrial function. Surprisingly, however we did not find any difference in ex vivo muscle function between any of the groups and their respective controls. Moreover, contrary to our hypothesis, we found no evidence of apoptosis (as measured by cell death) in skeletal muscle of mice treated with the TSP-1 mimetic, suggesting that losses in skeletal muscle capillary are not mediated by apoptosis driven by the TSP-1/CD36 pathway.

\section{Skeletal muscle mETC activity is altered by TSP-1}

TSP-1 has recently been identified as a potential mediator of mitochondrial biogenesis [13]. While both TSP-1 KO and CD47 KO animals have been reported to have increased mitochondrial density, as well as evidence of increased markers of mitochondrial biogenesis [13], these are the first data examining the functional measures of mitochondrial respiration when stimulating the TSP-1-CD36 receptor pathway. Although, we and others have previously 
shown that CD36 is important in controlling capillary growth [7, 9, 26, 48-50], and it has also been shown that CD36 is found on the mitochondrial membrane [51], these are the first data to suggest the TSP-1/CD36 pathway may also influence mitochondrial activity. Indeed, we find that pharmacological overexpression of the TSP-1 mimetic ABT-510 significantly decreased complex III and IV mETC activity, and to some extent complex I activity as well. It could be that the decrease in mETC activity is due to a decrease in mitochondria number associated with an increase in TSP-1. This decrease could be driven by a decrease in oxygen delivery, due to fewer capillaries, as hypoxia has been shown to decrease mitochondrial content $[19,52]$.

Supporting this observation is the finding that TSP-1 KO mice, which have been reported to have elevated muscle capillarity [10], displayed an opposite trend for greater complex IV mETC activity compared to WT mice. These data suggest that TSP-1 can influence mitochondrial biogenesis using the CD36 pathway, in addition to the CD47 pathway, but further studies will be needed to better understand the individual effects and/or differential contributions of these two TSP-1 receptor pathways.

The fact that the GA and SOL were more affected than the PLT could point to a fiber type specific response. That is, the SOL and the deep portion of the GA are both primarily oxidative, while the PLT is primarily glycolytic in nature. Oxidative fibers have been shown to have high levels of mitochondria density, so it could be that these regions are affected first. However, previously TSP-1 has been shown to decrease mitochondria in the quadriceps which is a primarily glycolytic muscle [13]. Additional analysis of fiber type will need to be addressed to answer this question.

\section{Ex vivo Muscle Function is not different in TSP-1 manipulated mice}


Because mitochondria are essential for sustaining aerobic exercise we hypothesized that muscle fatigue would be increased in animals subjected to a TSP-1 mimetic, and decreased in mice lacking TSP-1. However we found that ex vivo muscle fatigue remained unaltered in TSP$1 \mathrm{KO}$ and TSP-1 mimetic treated mice. The lack of difference in ex vivo muscle function and fatigue could point to the muscle vascular bed as the potential primary cause for the decrease in whole body exercise capacity seen previously in response to TSP-1 [53], and not the decrease in mitochondria function per se. Our findings here suggests that even with decreased mitochondrial activity, the muscles can still function normally provided that it has unlimited substrate (as in the ex vivo preparation).

\section{Short term chronic exposure to a TSP-1 mimetic does not increase cell death}

In earlier work we showed that capillarity was decreased with 14-day delivery of ABT510 [9]. Despite the reduction in capillarity, we did not see evidence of apoptosis with muscle TUNEL staining in those mice. This was counter to our hypothesis, and the prevailing theory that apoptosis is the principle mechanism by which capillary regression occurs in skeletal muscle vascular remodeling $[20,24,35,37,54]$. While we speculated that apoptosis might have occurred at an earlier time point, we were again surprised to still find no evidence of cell death with either 3 or 7 day treatment with ABT-510. These data imply that the reduction in muscle capillarity that occurs with chronic exposure to a TSP-1 mimetic is not mediated by a traditional cell death mechanism (e.g. apoptosis).

One hypothesis that could be made is that the capillary network is being effectively shrunk, without removal of endothelial cells. Indeed, it has been shown previously that there is capillary recruitment with exercise stimulus, so it could be that recruitment or vascular availability is decreased under the ABT-510 exposure [55-58]. However, it is likely that our 
methodology used to stain for capillaries would still show these intact, but non-functional networks. It could be that the mimetic is causing high levels of autophagy, resulting in ultimate cell dysfunction and death. Autophagy has been shown to be important in other systems, and in skeletal muscle [59-61]. While not directly linked to CD36, recently TSP-1/CD47 directed mechanisms have been shown to increase autophagy in cancer cells without causing apoptosis [62]. It has also been shown that other anti-angiogenic proteins, such as endostatin and K5, can trigger autophagy, which results in inhibition of angiogenesis [63]. While only speculative at this point, this could be a non-apoptotic mechanism for the removal of endothelial cells in skeletal muscle, or reduction in their viability, and hence a resultant shrinking of the capillary network.

The lack of cell death in the whole skeletal muscle leads to the conclusion that the TSP-1 mimetic has no effect on the myocyte apoptosis, as suggested previously [9]. This also agrees with our functional data showing no difference in both contractile characteristics and fatigue in the SOL and EDL muscles of those given a 14 day dosage of ABT-510.

\section{Summary}

In summary, we found that ABT-510 does not increase cell death in 3 and 7 day chronic exposures. However it does decrease mitochondrial electron transport chain enzyme activities, whereas the opposite appears true for mice lacking TSP-1, which have a trend for an increase in electron transport enzyme activities. Surprisingly no change in ex vivo muscle function was found to occur in association with the decrease ETC activity. Nevertheless, these data show that in addition to TSP-1 anti-angiogenic effects, it can also influence mitochondrial respiration 
activity through its CD36 receptor pathway. The importance of this finding and what component this plays in muscle function, and/or whole body exercise, remains to be elucidated. 


\section{Acknowledgements}

The authors would like to acknowledge Kyle Mandler, Ge Guo, and Cody Nichols for their contribution to the data in this manuscript.

\section{Conflict of interest}

The authors report no conflict of interest 


\section{Citations}

1. Jimenez, B., et al., Signals leading to apoptosis-dependent inhibition of neovascularization by thrombospondin-1. Nature medicine, 2000. 6(1): p. 41-8.

2. Lange-Asschenfeldt, B., et al., Increased and prolonged inflammation and angiogenesis in delayed-type hypersensitivity reactions elicited in the skin of thrombospondin-2deficient mice. Blood, 2002. 99(2): p. 538-545.

3. Varma, V., et al., Thrombospondin-1 Is an Adipokine Associated With Obesity, Adipose Inflammation, and Insulin Resistance. Diabetes, 2008. 57(2): p. 432-439.

4. Ridnour, L.A., et al., Nitric oxide regulates angiogenesis through a functional switch involving thrombospondin-1. Proceedings of the National Academy of Sciences of the United States of America, 2005. 102(37): p. 13147-13152.

5. Kvansakul, M., J.C. Adams, and E. Hohenester, Structure of a thrombospondin Cterminal fragment reveals a novel calcium core in the type 3 repeats. EMBO J, 2004. 23(6): p. 1223-1233.

6. Chen, H., M.E. Herndon, and J. Lawler, The cell biology of thrombospondin-1. Matrix Biology, 2000. 19(7): p. 597-614.

7. Febbraio, M., D.P. Hajjar, and R.L. Silverstein, CD36: a class B scavenger receptor involved in angiogenesis, atherosclerosis, inflammation, and lipid metabolism. Journal of Clinical Investigation, 2001. 108(6): p. 785-792.

8. Primo, L., et al., Identification of CD36 molecular features required for its in vitro angiostatic activity. The FASEB journal, 2005. 19(12): p. 1713-1715.

9. Audet, G.N., et al., Chronic delivery of a thrombospondin-1 mimetic decreases skeletal muscle capillarity in mice. PLoS One, 2013. 8(2): p. e55953.

10. Malek, M.H. and I.M. Olfert, Global deletion of thrombospondin-1 increases cardiac and skeletal muscle capillarity and exercise capacity in mice. Experimental physiology, 2009. 94(6): p. 749-760.

11. Roudier, E., et al., Angio-adaptation in unloaded skeletal muscle: new insights into an early and muscle type-specific dynamic process. The Journal of Physiology, 2010.

588(22): p. 4579-4591.

12. Olfert, I.M., et al., Temporal thrombospondin-1 mRNA response in skeletal muscle exposed to acute and chronic exercise. Growth Factors, 2006. 24(4): p. 253-259.

13. Frazier, E.P., et al., Age-dependent regulation of skeletal muscle mitochondria by the thrombospondin-1 receptor CD47. Matrix Biology, 2011.

14. Isenberg, J., W. Frazier, and D. Roberts, Thrombospondins: from structure to therapeutics: Thrombospondin-1: a physiological regulator of nitric oxide signaling. Cellular and Molecular Life Sciences CMLS, 2008. 65(5): p. 728-742.

15. Isenberg, J.S., et al., CD47 is necessary for inhibition of nitric oxide-stimulated vascular cell responses by thrombospondin-1. Journal of Biological Chemistry, 2006. 281(36): p. 26069-26080.

16. Isenberg, J.S., et al., Thrombospondin-1 inhibits endothelial cell responses to nitric oxide in a cGMP-dependent manner. Proceedings of the National Academy of Sciences of the United States of America, 2005. 102(37): p. 13141.

17. Wagner, P.D., Determinants of maximal oxygen transport and utilization. Annual review of physiology, 1996. 58(1): p. 21-50. 
18. Hoppeler, H. and M. Vogt, Muscle tissue adaptations to hypoxia. Journal of Experimental Biology, 2001. 204(18): p. 3133-3139.

19. Hoppeler, H., et al., Response of skeletal muscle mitochondria to hypoxia. Experimental physiology, 2003. 88(1): p. 109-119.

20. Friedl, P., P. Vischer, and M. Freyberg, The role of thrombospondin-1 in apoptosis. Cellular and molecular life sciences, 2002. 59(8): p. 1347-1357.

21. Guo, N., et al., Thrombospondin 1 and type I repeat peptides of thrombospondin 1 specifically induce apoptosis of endothelial cells. Cancer research, 1997. 57(9): p. 1735.

22. Miao, W.-M., et al., Thrombospondin-1 Type 1 Repeat Recombinant Proteins Inhibit Tumor Growth through Transforming Growth Factor- $\beta$-dependent and-independent Mechanisms. Cancer research, 2001. 61(21): p. 7830-7839.

23. Nör, J., et al., Thrombospondin-1 induces endothelial cell apoptosis and inhibits angiogenesis by activating the caspase death pathway. Journal of vascular research, 2000. 37(3): p. 209-218.

24. Rege, T.A., et al., Thrombospondin-1-induced apoptosis of brain microvascular endothelial cells can be mediated by TNF-R1. Journal of cellular physiology, 2009. 218(1): p. 94-103.

25. Haviv, F., et al., Thrombospondin-1 mimetic peptide inhibitors of angiogenesis and tumor growth: design, synthesis, and optimization of pharmacokinetics and biological activities. Journal of medicinal chemistry, 2005. 48(8): p. 2838-2846.

26. Dawson, D.W., et al., CD36 mediates the in vitro inhibitory effects of thrombospondin-1 on endothelial cells. The Journal of cell biology, 1997. 138(3): p. 707.

27. Anderson, J.C., et al., ABT-510, a modified type 1 repeat peptide of thrombospondin, inhibits malignant glioma growth in vivo by inhibiting angiogenesis. Cancer biology \& therapy, 2007. 6(3): p. 454.

28. Greenaway, J., et al., ABT-510 induces tumor cell apoptosis and inhibits ovarian tumor growth in an orthotopic, syngeneic model of epithelial ovarian cancer. Molecular cancer therapeutics, 2009. 8(1): p. 64.

29. Hoekstra, R., et al., Phase I Safety, Pharmacokinetic, and Pharmacodynamic Study of the Thrombospondin-1-Mimetic Angiogenesis Inhibitor ABT-510 in Patients With Advanced Cancer. Journal of Clinical Oncology, 2005. 23(22): p. 5188-5197.

30. Rusk, A., et al., Preclinical evaluation of antiangiogenic thrombospondin-1 peptide mimetics, ABT-526 and ABT-510, in companion dogs with naturally occurring cancers. Clinical cancer research, 2006. 12(24): p. 7444.

31. Yap, R., et al., Metronomic low-dose chemotherapy boosts CD95-dependent antiangiogenic effect of the thrombospondin peptide ABT-510: a complementation antiangiogenic strategy. Clinical cancer research, 2005. 11(18): p. 6678.

32. Campbell, N.E., et al., The Thrombospondin-1 Mimetic ABT-510 Increases the Uptake and Effectiveness of Cisplatin and Paclitaxel in a Mouse Model of Epithelial Ovarian Cancer, Neoplasia Press Inc.

33. Isenberg, J.S., C. Yu, and D.D. Roberts, Differential effects of ABT-510 and a CD36binding peptide derived from the type 1 repeats of thrombospondin-1 on fatty acid uptake, nitric oxide signaling, and caspase activation in vascular cells. Biochemical Pharmacology, 2008. 75(4): p. 875-882.

34. Meeson, A.P., et al., VEGF deprivation-induced apoptosis is a component of programmed capillary regression. Development, 1999. 126(7): p. 1407-1415. 
35. de Resende, M.M., et al., Role of endothelial cell apoptosis in regulation of skeletal muscle angiogenesis during high and low salt intake. Physiological Genomics, 2006. 25(2): p. 325-335.

36. Tang, K., et al., Capillary regression in vascular endothelial growth factor-deficient skeletal muscle. Physiological Genomics, 2004. 18(1): p. 63-69.

37. Fujino, H., et al., Regression of capillary network in atrophied soleus muscle induced by hindlimb unweighting. Journal of Applied Physiology, 2005. 98(4): p. 1407-1413.

38. Hasina, R., et al., ABT-510 is an effective chemopreventive agent in the mouse 4nitroquinoline 1-oxide model of oral carcinogenesis. Cancer Prevention Research, 2009. 2(4): p. 385.

39. Joyce, D., et al., Evaluation of the Thrombospondin-1 analogue ABT-510 in the APCMin/+ mouse intestinal adenoma model. Journal of Clinical Oncology, 2006. 24(18_suppl): p. 13545.

40. Pistilli, E.E., et al., Targeting the Activin Type IIB Receptor to Improve Muscle Mass and Function in the mdx Mouse Model of Duchenne Muscular Dystrophy. Am J Pathol, 2011. 178(3): p. 1287-97.

41. Burke, R.E., et al., Physiological types and histochemical profiles in motor units of the cat gastrocnemius. J Physiol, 1973. 234(3): p. 723-48.

42. Brooks, S.V. and J.A. Faulkner, Contractile properties of skeletal muscles from young, adult and aged mice. J Physiol, 1988. 404: p. 71-82.

43. Lynch, G.S., et al., Force and power output of fast and slow skeletal muscles from mdx mice 6-28 months old. J Physiol, 2001. 535(Pt 2): p. 591-600.

44. Dabkowski, E.R., et al., Diabetic cardiomyopathy-associated dysfunction in spatially distinct mitochondrial subpopulations. American Journal of Physiology-Heart and Circulatory Physiology, 2009. 296(2): p. H359-H369.

45. Trounce, I.A., et al., Assessment of mitochondrial oxidative phosphorylation in patient muscle biopsies, lymphoblasts, and transmitochondrial cell lines. Methods in enzymology, 1996. 264: p. 484.

46. Rodriguez-Bies, E., et al., Muscle physiology changes induced by every other day feeding and endurance exercise in mice: effects on physical performance. PLoS One, 2010. 5(11): p. e13900.

47. Bradford, M.M., A rapid and sensitive method for the quantitation of microgram quantities of protein utilizing the principle of protein-dye binding. Analytical biochemistry, 1976. 72(1): p. 248-254.

48. Koch, M., et al., CD36-mediated activation of endothelial cell apoptosis by an $\mathrm{N}$ terminal recombinant fragment of thrombospondin-2 inhibits breast cancer growth and metastasis in vivo. Breast Cancer Res Treat, 2011. 128(2): p. 337-46.

49. Primo, L., et al., Identification of CD36 molecular features required for its in vitro angiostatic activity. The FASEB journal, 2005.

50. Zhang, X., et al., Thrombospondin-1 modulates vascular endothelial growth factor activity at the receptor level. The FASEB journal, 2009. 23(10): p. 3368-3376.

51. Bezaire, V., et al., Identification of fatty acid translocase on human skeletal muscle mitochondrial membranes: essential role in fatty acid oxidation. American Journal of Physiology-Endocrinology And Metabolism, 2006. 290(3): p. E509-E515. 
52. Sullivan, S.M. and R.N. Pittman, Relationship between mitochondrial volume density and capillarity in hamster muscles. American Journal of Physiology - Heart and Circulatory Physiology, 1987. 252(1): p. H149-H155.

53. Malek, M.H., et al., Similar skeletal muscle angiogenic and mitochondrial signalling following 8 weeks of endurance exercise in mice: discontinuous versus continuous training. Experimental physiology, 2013. 98(3): p. 807-818.

54. Dimmeler, S. and A.M. Zeiher, Endothelial Cell Apoptosis in Angiogenesis and Vessel Regression. Circulation research, 2000. 87(6): p. 434-439.

55. Hudlicka, O., B. Zweifach, and K. Tyler, Capillary recruitment and flow velocity in skeletal muscle after contractions. Microvascular research, 1982. 23(2): p. 201-213.

56. Heinonen, I.H., et al., Regulation of human skeletal muscle perfusion and its heterogeneity during exercise in moderate hypoxia. American Journal of PhysiologyRegulatory, Integrative and Comparative Physiology, 2010. 299(1): p. R72-R79.

57. Honig, C.R., C.L. Odoroff, and J.L. Frierson, Capillary recruitment in exercise: rate, extent, uniformity, and relation to blood flow. American Journal of Physiology-Heart and Circulatory Physiology, 1980. 238(1): p. H31-H42.

58. Bourdillon, N., et al., Non-invasive evaluation of the capillary recruitment in the human muscle during exercise in hypoxia. Respiratory physiology \& neurobiology, 2009. 165(2): p. 237-244.

59. Kim, Y.A., et al., Autophagic response to exercise training in skeletal muscle with age. Journal of Physiology and Biochemistry, 2013: p. 1-9.

60. Marino, G. and C. Lopez-Otin, Autophagy: molecular mechanisms, physiological functions and relevance in human pathology. Cellular and Molecular Life Sciences CMLS, 2004. 61(12): p. 1439-1454.

61. Klionsky, D.J. and S.D. Emr, Autophagy as a regulated pathway of cellular degradation. Science, 2000. 290(5497): p. 1717-1721.

62. Kalas, W., et al., Thrombospondin-1 receptor mediates autophagy of RAS-expressing cancer cells and triggers tumour growth inhibition. Anticancer Res, 2013. 33(4): p. 142938.

63. Ramakrishnan, S., et al., Autophagy and angiogenesis inhibition. Autophagy, 2007. 3(5): p. 511-514. 


\section{Table 1.}

\begin{tabular}{|l|c|c|c|c|c|c|c|c|}
\hline & 3-CON & 3-ABT & 7-CON & 7-ABT & 14-CON & 14-ABT & WT & TSP-1 KO \\
\hline Age (Days) & $81 \pm 0$ & $81 \pm 0$ & $102 \pm 0$ & $102 \pm 0$ & $82 \pm 5$ & $90 \pm 6$ & $94 \pm 0$ & $94 \pm 1$ \\
\hline Body Mass (g) & $25.7 \pm 0.7$ & $25.4 \pm 0.3$ & $28.3 \pm 0.6$ & $28.6 \pm 0.4$ & $27.5 \pm 1.2$ & $27.1 \pm 0.9$ & $22.8 \pm 1.7$ & $25.3 \pm 1.2$ \\
\hline GA (mg) & $139 \pm 4$ & $135 \pm 2$ & $146 \pm 5$ & $146 \pm 3$ & $137 \pm 5$ & $141 \pm 4$ & $133 \pm 16$ & $116 \pm 5$ \\
\hline PLT (mg) & $19.0 \pm 0.6$ & $18.0 \pm 0.4$ & $21.0 \pm 0.4$ & $21.0 \pm 0.8$ & $19.7 \pm 0.9$ & $19.0 \pm 0.5$ & $14.66 \pm 0.8$ & $14.59 \pm 1.3$ \\
\hline SOL (mg) & $8.0 \pm 0.4$ & $7.0 \pm .0 .6$ & $10.0 \pm 0.2$ & $9.0 \pm 0.3$ & $7.7 \pm 0.5$ & $7.8 \pm 0.5$ & $9.4 \pm 1.4$ & $7.6 \pm 0.4$ \\
\hline GA/BM (mg $\cdot \mathbf{g - 1})$ & $5.0 \pm 0.01$ & $5.0 \pm 0.10$ & $5.0 \pm 0.01$ & $5.0 \pm .07$ & $5.0 \pm 0.1$ & $5.2 \pm 0.1$ & $5.8 \pm 0.4$ & $4.6 \pm 0.1 *$ \\
\hline PLT/BM (mg $\cdot \mathbf{g - 1})$ & $0.70 \pm 0.02$ & $0.70 \pm 0.02$ & $0.67 \pm 0.05$ & $0.70 \pm 0.01$ & $0.72 \pm 0.04$ & $0.70 \pm 0.02$ & $0.65 \pm 0.05$ & $0.57 \pm 0.04 *$ \\
\hline SOL/BM (mg $\cdot \mathbf{g - 1})$ & $0.30 \pm .01$ & $0.30 \pm 0.02$ & $0.36 \pm 0.05$ & $0.30 \pm 0.01$ & $0.29 \pm 0.01$ & $0.28 \pm 0.01$ & $0.41 \pm 0.04$ & $0.31 \pm 0.02$ \\
\hline
\end{tabular}

All values \pm SE. $* \mathrm{P} \leq 0.05$ from $\mathrm{WT}$ 
Table 2: Mitochondrial Electron Transport Enzyme Activities

\begin{tabular}{|l|c|c|c|c|}
\cline { 2 - 5 } \multicolumn{1}{c|}{} & Vehicle & ABT-510 & WT & TSP-1 KO \\
\hline Complex I & $26 \pm 4$ & $17 \pm 2 \dagger$ & $19 \pm 2$ & $16 \pm 1$ \\
\hline Complex III & $59 \pm 5$ & $42 \pm 6 *$ & $49 \pm 5$ & $42 \pm 3$ \\
\hline Complex IV & $62 \pm 11$ & $36 \pm 5 *$ & $91 \pm 8$ & $131 \pm 18 \dagger \dagger$ \\
\hline
\end{tabular}

All Values \pm SE. $* \mathrm{P}<0.05$ compared to Vehicle; $\uparrow \mathrm{P}<0.07$ compared to Vehicle; $\uparrow \uparrow \mathrm{P}<0.07$ compared to WT 
Table 3: Anatomical and physiological characters of the EDL

\begin{tabular}{|l|l|l|l|l|}
\hline EDL & Vehicle & ABT-510 & WT & TSP-1 KO \\
\hline $\begin{array}{l}\text { Muscle Mass } \\
(\mathrm{mg})\end{array}$ & $11.40 \pm 0.33$ & $11.06 \pm 0.38$ & $9.99 \pm 0.84$ & $10.31 \pm 0.48$ \\
\hline CSA $\left(\mathrm{mm}^{2}\right)$ & $2.03 \pm 0.05$ & $2.00 \pm 0.09$ & $1.90 \pm 0.10$ & $1.90 \pm 0.12$ \\
\hline $\mathrm{L}_{0}(\mathrm{~mm})$ & $11.76 \pm 0.22$ & $11.61 \pm 0.23$ & $10.96 \pm 0.50$ & $11.46 \pm 0.27$ \\
\hline $\begin{array}{l}\text { Contraction } \\
\text { time (ms) }\end{array}$ & $20.0 \pm 3.1$ & $23.8 \pm 1.8$ & $23.8 \pm 1.8$ & $25.7 \pm 2.0$ \\
\hline $\begin{array}{l}1 / 2 \text { relaxation } \\
\text { time (ms) }\end{array}$ & $37.14 \pm 1.8$ & $37.50 \pm 1.64$ & $41.25 \pm 2.27$ & $37.14 \pm 2.85$ \\
\hline
\end{tabular}

All Values \pm SE. * $\mathrm{P}<0.05$ compared to Vehicle; $\dagger \mathrm{P}<0.07$ compared to Vehicle; $\uparrow \uparrow \mathrm{P}<0.07$ compared to WT 
Table 4: Anatomical and physiological characters of the soleus

\begin{tabular}{|l|l|l|l|l|}
\hline SOLEUS & Vehicle & ABT-510 & WT & TSP-1 KO \\
\hline $\begin{array}{l}\text { Muscle Mass } \\
(\mathrm{mg})\end{array}$ & $9.06 \pm 0.42$ & $8.61 \pm 0.31$ & $7.30 \pm 0.38$ & $8.63 \pm 0.38$ \\
\hline CSA $\left(\mathrm{mm}^{2}\right)$ & $1.26 \pm 0.06$ & $1.18 \pm 0.04$ & $1.80 \pm 0.10$ & $2.13 \pm 0.18$ \\
\hline \begin{tabular}{l}
$\mathrm{L}_{0}(\mathrm{~mm})$ \\
\hline $\begin{array}{l}\text { Contraction } \\
\text { time (ms) }\end{array}$
\end{tabular} & $9.81 \pm 0.16$ & $9.85 \pm 0.16$ & $8.50 \pm 0.23$ & $7.7 \pm 0.44$ \\
\hline $\begin{array}{l}1 / 2 \text { relaxation } \\
\text { time (ms) }\end{array}$ & $80 \pm 7$ & $41.3 \pm 3.0$ & $52.5 \pm 2.5$ & $36.7 \pm 6.7$ \\
\hline
\end{tabular}

All Values \pm SE. * $\mathrm{P}<0.05$ compared to Vehicle; $\uparrow \mathrm{P}<0.07$ compared to Vehicle; $\uparrow \uparrow \mathrm{P}<0.07$ compared to WT 


\section{Figure Legends}

Figure 1: 3 \& 7 days of ABT-510 treatment does not increase cell death. ABT-510 animals were given $30 \mathrm{mg} / \mathrm{Kg} /$ day systemic dose delivered by mini-osmotic pump, while vehicle animals received 5\% dextrose. Cell death was measured from 100ug of total protein by Cell Death ELISA. Values reported at A.U. absorbance values divided by total protein. $\mathrm{N}=4$ /group 3 day exposure; $\mathrm{N}=10$ /group 7 day exposure.

Figure 2: There is a trend for lower peak tetanus force in the SOL of ABT-510 treated animals. While not reaching statistical significance, there was a trend for lower peak tetanus $(\mathrm{P}=0.06)$ and peak tetanus force/fiber cross sectional area $(\mathrm{CSA})(\mathrm{P}=0.11)$ forces of 14 day ABT510 treated animals in the SOL muscle. There was no difference in EDL isometrics between groups (not shown).

Figure 3: No change in ex vivo muscle fatigue between vehicle and ABT-510 treated mice. There is no difference between the fatigue curves of 14 day ABT-510 and vehicle treated animals in either the EDL or SOL muscle. Open Boxes; Vehicle. Closed Diamonds; ABT-510.

Figure 4: No change in ex vivo muscle fatigue between WT and TSP-1 KO treated mice. There is no difference between the fatigue curves of TSP-1 KO and WT animals in either the EDL or SOL muscle. Open Circles; WT. Closed Triangles; KO. 
Figure 1

3 Day ABT-510 Implantation

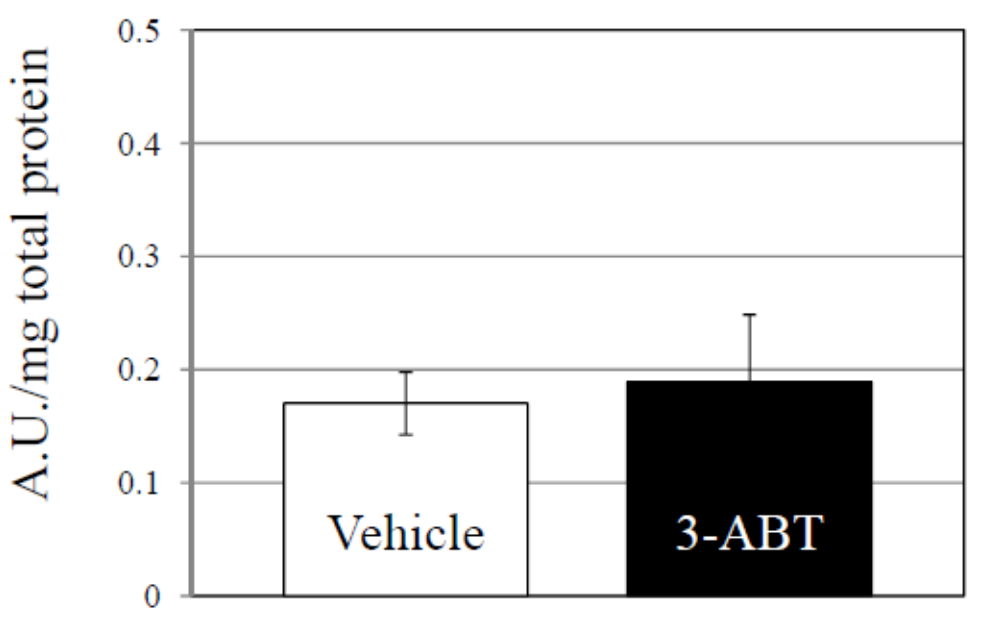

7 Day ABT-510 Implantation

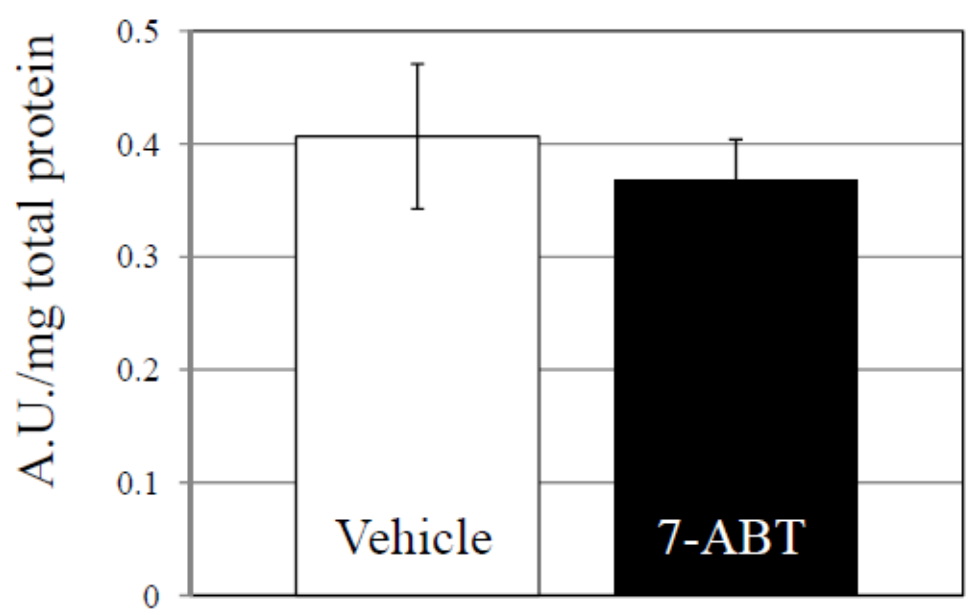


Figure 2
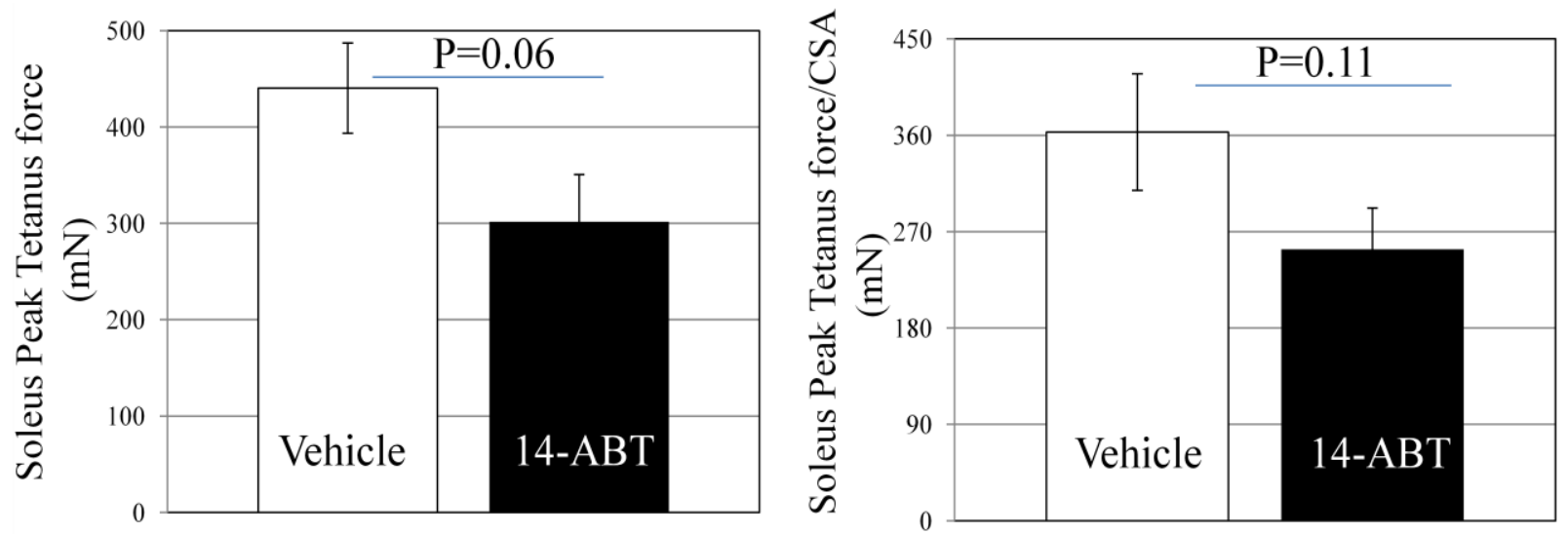
Figure 3

EDL
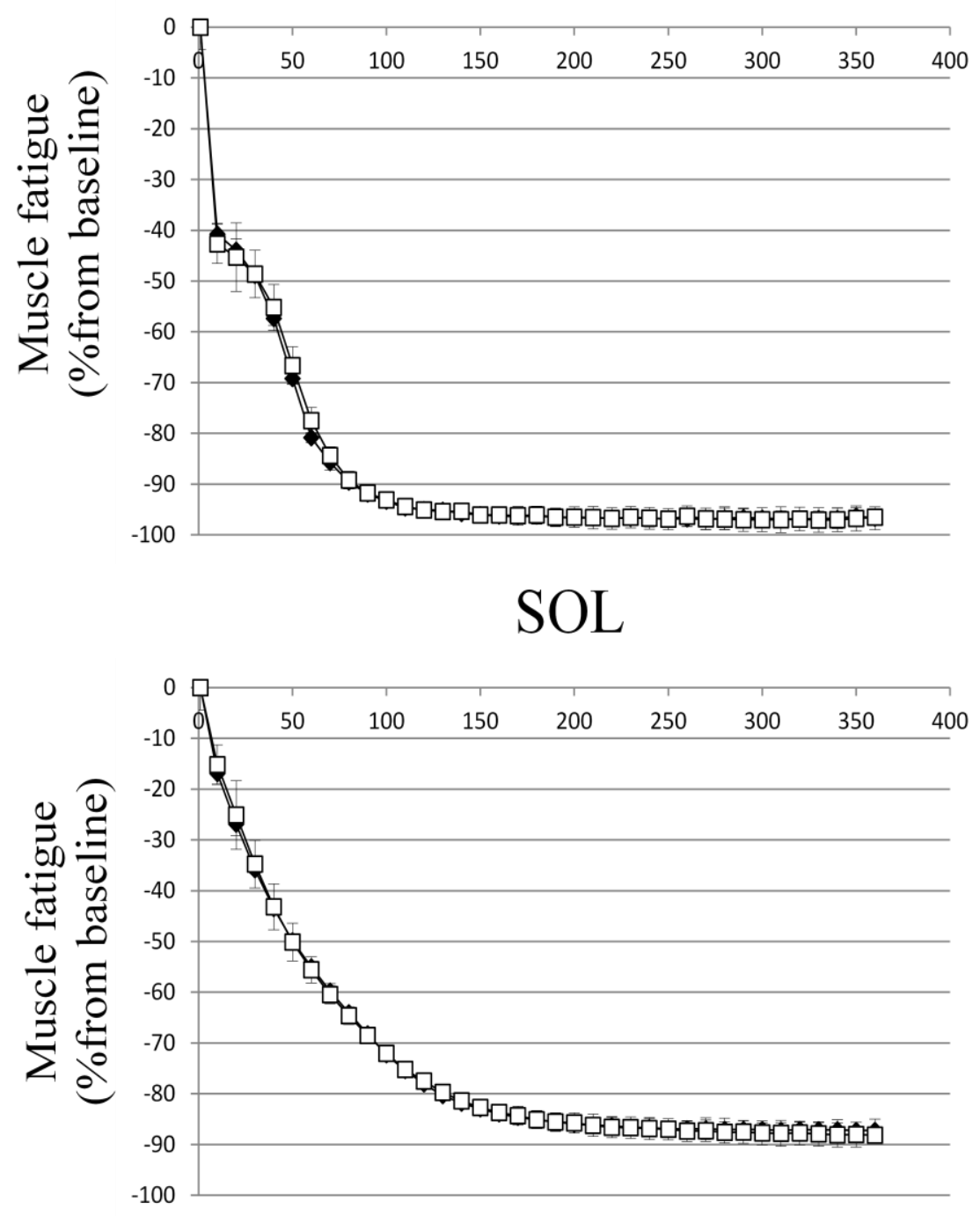
Figure 4

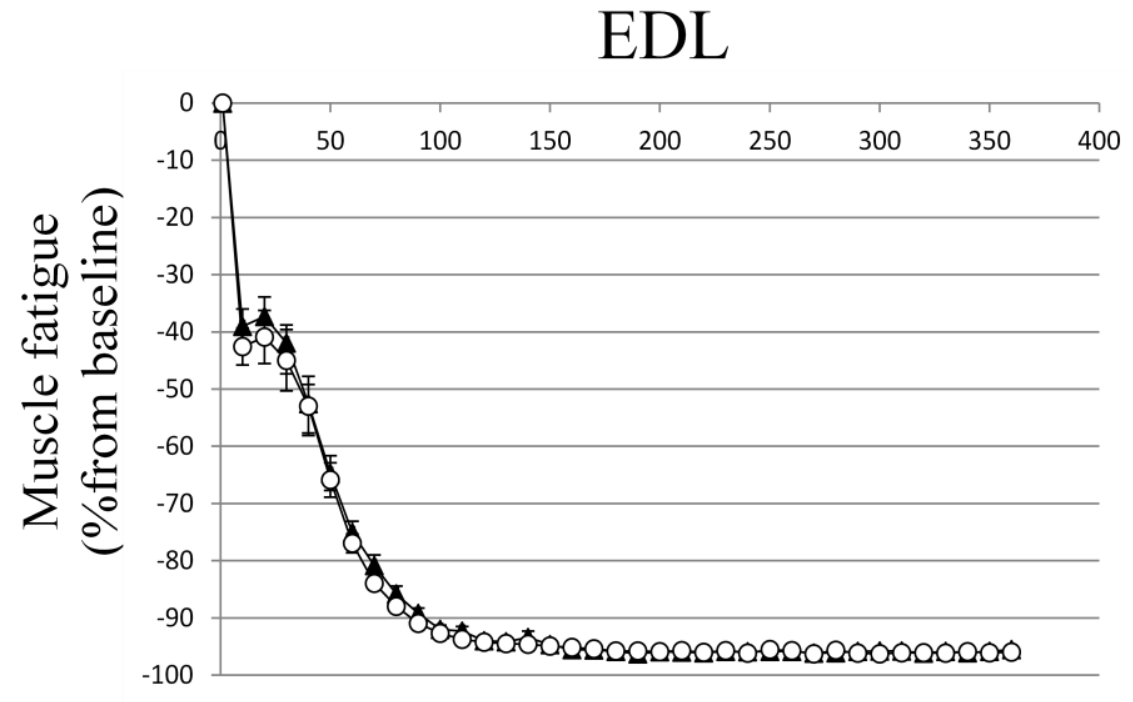

SOL

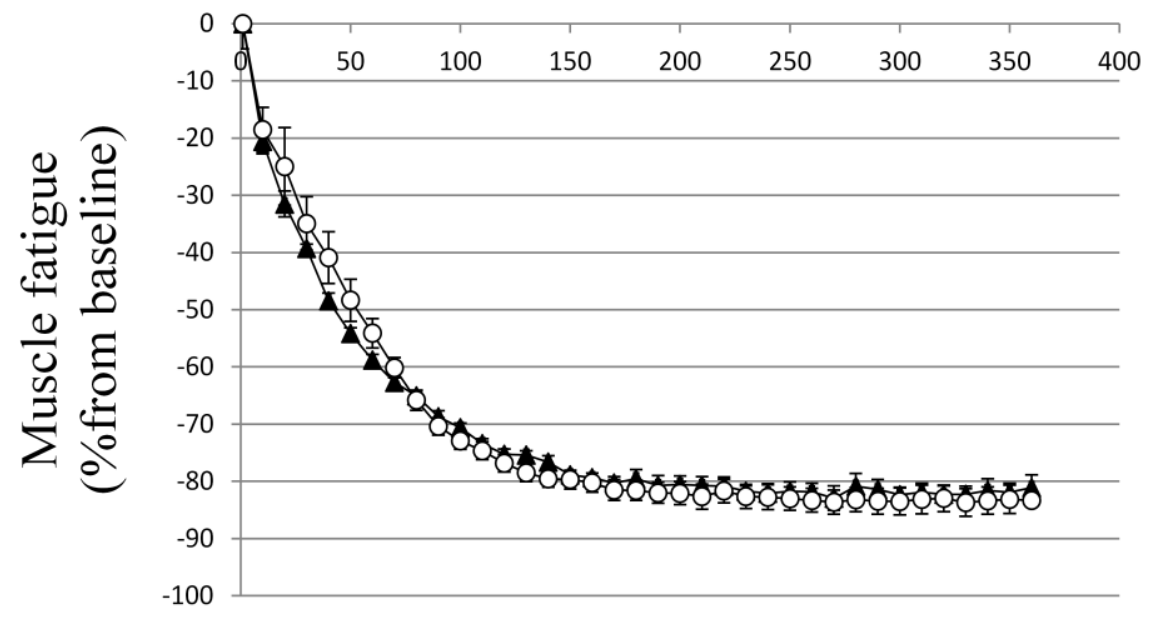


This page intentionally left blank 


\section{Chapter 5: TNF $\alpha$ mediated inflammation decreases skeletal muscle capillarity in association with thrombospondin-2}




\section{TNF $\alpha$ mediated inflammation decreases oxidative skeletal muscle capillarity in mice}

Gerald N Audet, W Kyle Mandler, Dharendra Thapa, Cody Nichols, John M. Hollander, I. Mark Olfert

West Virginia University School of Medicine, Center for Cardiovascular and Respiratory Sciences, Division of Exercise Physiology, Morgantown, WV, USA

Address for correspondence:

Mark Olfert, $\mathrm{PhD}$

West Virginia University School of Medicine

Center for Cardiovascular and Respiratory Sciences

Division of Exercise Physiology

PO Box 9105

Morgantown, WV 26508

Phone 304-293-7597

molfert@hsc.wvu.edu 


\section{This paper has been formatted for submission to the Journal of Applied Physiology}

\section{$\underline{\text { Abstract }}$}

Tumor necrosis factor alpha $(\mathrm{TNF} \alpha)$ is an important inflammatory cytokine that is implicated in several co-morbidities. High levels of systemic TNF $\alpha$ may be the link between chronic inflammation and skeletal muscle dysfunction found in many chronic diseases. Thrombospondin-family proteins (such as TSP-1 and TSP-2) are found in skeletal muscle and have been shown to be important in capillary rarefaction. Evidence from cell culture experiments also shows that TNF $\alpha$ may act to increase TSP-1 in a skeletal muscle specific manner. We hypothesized that a chronic increase in systemic TNF $\alpha$ would increase TSP-1 and/or TSP-2 resulting in capillary rarefaction and skeletal muscle dysfunction. We used a lungspecific TNFa overexpressing transgenic mouse model (TNF $\alpha+)$ which exhibits a chronic $\sim 300 \%$ increase in circulating TNF $\alpha$ levels to evaluate the effects of elevated circulating TNF $\alpha$ on skeletal muscle structure and function. Whole body endurance capacity was decreased by $120 \%$ in the $\mathrm{TNF} \alpha+$ animals, however in vivo muscle stimulated fatigue remained unchanged between $\mathrm{TNF} \alpha+$ and litter mate controls (CON). Capillarity was decreased by $16 \%$ in the soleus (SOL) of TNF $\alpha+$ animals $(\mathrm{P}<0.02)$, but no change was found in the plantaris (PLT), compared to CON. TSP-2, but not TSP-1, was significantly increased by $\sim 80 \%(\mathrm{p}<0.02)$ in muscle of $\mathrm{TNF} \alpha+$ compared to $\mathrm{CON}$. Mitochondrial electron transport chain enzyme activity for complex III increased by $170 \%$ in the SOL, while complex IV activity increased $74 \%$ in the PLT of $\mathrm{TNF} \alpha+$ compared to $\mathrm{CON}(\mathrm{P}<0.05)$. No evidence of altered reactive oxygen species was found. These data suggest that chronic systemic elevation of TNF $\alpha$ can alter TSP- 2 which could contribute to losses in skeletal muscle capillary. Reducing systemic $\mathrm{TNF} \alpha+$ may serve as a therapeutic target to reduce skeletal muscle dysfunction associated with capillary rarefaction. 


\section{$\underline{\text { Introduction }}$}

Tumor necrosis factor alpha $(\mathrm{TNF} \alpha)$ is an important inflammatory cytokine that is implicated in the co-morbidities, such as skeletal muscle dysfunction, of many chronic diseases [1-6]. For example, associations between elevated systemic TNF $\alpha$ and muscle dysfunction are often found with chronic obstructive pulmonary disease (COPD), chronic heart failure, and diabetes $[2,4,6-10]$ Interestingly, in a mouse model of TNF $\alpha$ overexpression (TNF $\alpha+)$, Zuo et al. have shown increased soleus (SOL) muscle fatigue and disrupted contractile kinetics in the extensor digitorum longus (EDL) of TNF $\alpha+$ mice [11]. Tang et al. [12] also showed that in situ muscle endurance was decreased in this same mouse model. In humans, COPD is also well known to decrease skeletal muscle exercise capacity, which is also associated with an increased circulating level of TNF $\alpha$. It has been proposed that skeletal muscle capillary loss in chronic disease could be a significant contributor to overall skeletal muscle dysfunction and comorbidity [2, 13-18]. The mechanism(s) by which increased circulating TNF $\alpha$ may disrupt skeletal muscle function and/or capillarity are undetermined.

Angiogenesis is controlled by both positive and negative angiogenic proteins [19-21]. There is growing evidence that negative angiogenic regulators, such as the thrombospondins [21$26]$ and endostatin $[21,27,28]$, are critical microvessel regulators in both pathological and physiological skeletal muscle angiogenesis. Thrombospondin (TSP)-1 and TSP-2 are large extracellular matrix multi-domain proteins that inhibit angiogenesis in vitro and in vivo [29-33]. They share multiple binding receptors, such as CD36, CD47 and LRP-1, which inhibit endothelial cell proliferation, migration, tube formation, and induce apoptosis [29, 30, 32, 34]. TSP-1 appears to play an important role in skeletal muscle capillary rarefaction occurring with hindlimb unloading [25] and has been implicated as an important regulator of whole body 
exercise capacity through its powerful anti-angiogenic actions [24]. The role of TSP-2 in regulating skeletal muscle capillarity and its effects on exercise capacity are less well studied.

Evidence from cell culture experiments have shown that TNF $\alpha$ can act to increase TSP-1 in a myoblast specific manner [35], and increased systemic TNF $\alpha$ has been shown to increase circulating TSP-1 protein levels in humans [36]. Interestingly, TNF $\alpha$ knockout mice also display increased running capacity compared to controls [37]. It is tempting to speculate that the absence of TNF $\alpha$ might lower basal expression of TSP-1 and/or TSP-2 and thereby increase skeletal muscle capillarity that could explain the greater capacity for exercise. Collectively these data hint to a potential mechanism that may link TNF $\alpha$ mediated chronic systemic inflammation to muscle expression of TSP-1/TSP-2, which could be responsible for skeletal muscle capillary rarefaction and muscle dysfunction [38].

In this study we examined the effects chronic systemic elevation of TNFa has on skeletal muscle structure and function. We used a lung specific TNF $\alpha$ overexpressing transgenic mouse model known to exhibit increased systemic TNFa. We hypothesized that chronic systemic elevation of circulating TNF $\alpha$ would increase expression of TSP-1 and/or TSP-2 in skeletal muscle, and therefore decrease skeletal muscle capillarity. We also hypothesized that elevated thrombospondin levels would lead to decreased in vivo muscle function, as well as impaired skeletal muscle mitochondrial function. 


\section{$\underline{\text { Methods }}$}

Animals: Lung specific TNF $\alpha$ overexpressing mice were obtained as a gift from Dr. Peter Wagner (University of California, San Diego). All procedures that involved animals were approved by the West Virginia University Institutional Animal Care and Use Committee. These mice use a surfactant protein-C promoter to overexpress TNF $\alpha$ in the lung [39]. Transgenic $\mathrm{TNF} \alpha$ overexpressing $\left(\mathrm{TNF}^{++}, \mathrm{n}=30\right)$ and littermate $\operatorname{control}(\mathrm{CON}, \mathrm{n}=25)$ mice were studied at 5-6 months of age.

\section{Whole body and in vivo skeletal muscle exercise testing}

Whole body exercise capacity was assessed on a sub-set of animals $(\mathrm{TNF} \alpha+\mathrm{N}=9, \mathrm{CON}$ $\mathrm{N}=7$ ). The exercise endurance test was performed at least 48 hours before any tissues were taken for morphometric or molecular analysis. This time frame was selected to ensure that the exercise bout did not influence basal protein levels in muscles harvested on the final study day [40]. Exercise capacity was measured using a commercially available treadmill (Columbus Instruments, Columbus, $\mathrm{OH})$. The mice were allowed to warm-up at 4 meters $/$ minute $(\mathrm{m} / \mathrm{min})$ for 5 minutes, at a 10 degree incline. Thereafter, the treadmill speed was gradually increased to $20 \mathrm{~m} / \mathrm{min}$ over $10 \mathrm{~min}$. The animals then ran as long as possible at $20 \mathrm{~m} / \mathrm{min}$ until they were exhausted or refused to run. Exhaustion was defined as the inability of the animal to maintains its normal running position on the moving belt of the treadmill, and/or more than 5 seconds spent on the shock grid (at the rear of the treadmill). Animals were continually encouraged to keep running by using noise from forced air jets and the electrical deterrence $(<0.2 \mathrm{~mA})$ via a shock grid at the rear of the treadmill. Total run time was recorded and used as an assessment of whole body exercise capacity. 
In vivo muscle fatigue in the hindlimb muscle of anesthetized mice was measured by stimulating the tibial nerve ( $\mathrm{TNF} \alpha+\mathrm{N}=15, \mathrm{CON} \mathrm{N}=7$ ). In vivo analysis was performed in an effort to keep the endogenous blood supply intact and most accurately reflect what is occurring in the conscious exercising animal. Briefly, mice were anesthetized with isoflourane $(2 \%)$ and the left knee was secured in flexion by placing a metal rod on the lateral side of the knee. The left foot was then secured to a footplate connected to a servomotor (Cambridge Technology Inc. Model 6350*350, Cambridge, MA). The ankle joint was then aligned with the axis of rotation of the servomotor and electrically evoked via the tibial nerve by insertion of platinum electrodes through the skin (Grass Medical Instruments). The electrodes were placed through the skin so that they were flanking either side of the nerve. The left plantar flexor muscle group from each animal was subjected to standardized pulses to determine correct electrode placement $(10 \mathrm{v}, 100$ $\mathrm{Hz}, 200 \mu$ s pulses). Once completed, the animals were subjected to stimulation protocol (12v, 40hz, 333ms, every second for 180 seconds) designed to mimic the modified Bruce protocol used in humans [41]. Fatigue was defined by a reduction to $60 \%$ of peak force during the protocol.

\section{Muslce Morphometry}

Hindlimb skeletal muscle was surgically excised and frozen in isopentane cooled by liquid nitrogen. Frozen tissue was cut using a $-20^{\circ} \mathrm{C}$ cyromicrotome (Jung-Reichert Cryocut 1800: Cambridge Instruments, Germany) to yield $10 \mu \mathrm{m}$ transverse sections. Great care was taken to ensure the cryosectioned muscles were cut along the transverse plane. Sections were stained for dipeptidly-peptidase IV (DPP IV) and alkaline phosphatase (AP) following the method of Lojda 
[42, 43]. A light microscope (Zeiss primo star, Zeiss, Oberkochen, Germany) was used to digitally acquire (Axiocam IC c3, Axiovision 4.8.2.0, Zeiss, Oberkochen, Germany) 20X images of the gastrocnemius, plantaris, and soleus. Capillary and myofiber counting was performed by a single individual blinded to group identification. For the gastrocnemius muscle, we obtained images in a checkerboard fashion across the entire muscle, thus both superficial and deep regions within the gastrocnemius could be included in the analysis. For the plantaris and soleus muscles, respectively, the entire muscle was imaged and analyzed. Counting was performed by visualization from acquired images using a custom program in MATLAB (version 7.0.0.27, The Mathworks, MA, USA) allowing the operator to visually mark and count the capillaries and fibers on each image. Capillary-to-fiber ratio (C:F, number of capillaries/number muscle fibers), capillary density (CD, number of capillaries $/ \mathrm{mm}^{2}$ muscle fiber area), and fiber cross sectional area (FCSA) were separately calculated for the gastrocnemius (GA), soleus (SOL), and plantaris (PLT) (N=19-30 images/muscle/group), (TNF $\alpha+\mathrm{N}=15 ; \mathrm{CON} N=7$ per muscle).

\section{Protein Preparation}

GA, SOL, and PLT muscles from each group were excised and flash frozen in liquid nitrogen. For molecular analysis, muscle were then separately homogenized in a lysis buffer containing 50 $\mathrm{mM}$ Tris/ $\mathrm{HCl}(\mathrm{pH} 7.4), 150 \mathrm{mM} \mathrm{NaCl}, 0.5 \%$ Triton $\mathrm{X}-100$, and protease inhibitors (Complete Tablet, Roche Applied Science, Indianapolis, IN). Homogenates were centrifuged at $4^{\circ} \mathrm{C}$, at $10,000 \mathrm{~g}$ for 10 minutes, and supernatants removed and placed in new tubes. For citrate synthase (CS) activity, 10-50mg of muscle, depending on size of muscle, was separately homogenized in a simple homogenization buffer $\left(175 \mathrm{mM} \mathrm{KCl}, 2 \mathrm{mM}\right.$ EDTA in $\left.\mathrm{DDH}_{2} \mathrm{O}\right)$ as described previously $[12,44]$. This was then spun for 1 minute at $8000 \mathrm{~g}$. These samples were then freeze-thawed 
several times to stabilize protein content. Blood samples were obtained from the heart and allowed to coagulate on ice. They were then centrifuged at $3000 \mathrm{~g}$ for 10 minutes and flash frozen in liquid nitrogen. Total protein was measured from all samples by Bradford assay (\#23236 Pierce Coomassie Plus Protein Assay Kit, Thermo Scientific, Rockford, IL) [45].

\section{$\underline{\text { Protein Analysis }}$}

An Angiogenic Protein Array was performed on 100ug of SOL muscle using a protein array kit per manufacturer's instructions (\#ARY015, R\&D Systems, Minneapolis, MN, USA). $(\mathrm{TNF} \alpha+\mathrm{N}=2 ; \mathrm{CON} N=2$ per muscle)

Quantification of VEGF was made from a total of $100 \mu \mathrm{g}$ of protein using a commercially available ELISA kit according to the manufactures instructions (\# MMV00, R\&D Systems, Minneapolis, MN, USA). All samples were run in duplicate. $(\mathrm{TNF} \alpha+\mathrm{N}=22$; $\mathrm{CON} N=13$ per muscle)

Quantification of TNF $\alpha$ was made from a total of either $50 \mu \mathrm{L}$ of serum or $100 \mu \mathrm{g}$ of muscle homogenate using a commercially available ELISA kit according to the manufactures instructions. All samples were run in duplicate. $(\mathrm{TNF} \alpha+\mathrm{N}=6, \mathrm{CON} N=6$ per muscle)

TSP-1 was analyzed via western blot. In brief, samples were separated on a 3-8\% SDSPAGE (NuPAGE Novex 3-8\% Tris-Acetate Midi Gel, Invitrogen, Burlington, ON, Canada) and blotted onto a $0.45 \mu \mathrm{m}$ nitrocellulose membrane (Pierce nitrocellulose membrane, Thermo Scientific, Rockford, IL). After blocking with 5\% fat-free milk, membranes were probed using antibodies against TSP-1 (1:500 \#59887, Santa Cruz, Dallas, TX, USA), $\beta$-tubulin (1:1000, \#2148, Cell Signaling), secondary HRP-conjugated anti-mouse (1:1000, \#p0260, Dako, GE Healthcare, Piscataway, NJ) and secondary HRP-conjugated anti-rabbit (1:1000, \#P0217, 
Dako). Proteins were visualized using chemiluminescence detection (Pierce ECL, Thermo Scientific, Rockford, IL) and digitally imaged (G:BOX Gel imager, Syngene, Cambridge, UK) using Genesnap software (Ver. 7.01, Syngene, Cambridge, UK). Equal protein loading was verified by immunodetection of $\beta$-tubulin as our loading control. Quantification of protein expression levels were carried out using NIH Image J Software (v1.62) and expressed as densitometric arbitrary units (AU). All blots were run in duplicate to confirm results. (TNF $\alpha+$ $\mathrm{N}=8, \mathrm{CON} \mathrm{N}=8$ per muscle)

\section{$\underline{\text { Reactive Oxygen Species }}$}

DCF Assay: Total reactive oxygen species was measure in fresh tissue homogenates by dichlorofluoroscein (DCF) as described previously, with some modification [46]. In brief, the dichlorofluoroscin acetate (DCFH-DA) stock solution was made fresh by dissolving 1.25mM of DCF in $100 \%$ ethanol and storing it in the dark at $-20^{\circ} \mathrm{C}$. DCFH-DA was dissolved in ethanol to aid in the transport across membranes. $2.5 \mu \mathrm{L}$ of PLT and $5 \mu \mathrm{L}$ of SOL homogenate were added to each well of a 96-well black walled plate containing $50 \mu \mathrm{L}$ of freshly made $0.1 \mathrm{M}$ phosphate buffer (pH 7.2), and $40 \mu \mathrm{l}$ of $1.25 \mathrm{mM}$ DCFH-DA (total volume 92.5 or $95 \mu \mathrm{L}$, SOL, PLT respectively). Samples were run in duplicate, and each plate contained 2 blanks containing the phosphate buffer and DCFH-DA but no protein to serve as background controls. The assay mixture was incubated for $20 \mathrm{~min}$ at $37^{\circ} \mathrm{C}$ to allow the DCFH-DA to cross any membranes. DCF formation was determined fluorometrically (FlexStation 3 plate reader, Molecular Devices, California, United States) at excitation wavelength of $488 \mathrm{~nm}$ and emission wavelength of 525 $\mathrm{nm}$ at $37^{\circ} \mathrm{C}$. Measurements were made every $10 \mathrm{~min}$ for $60 \mathrm{~min}$, and the linear DCF production 
rate was determined relative to the amount of protein added to each well. $(\mathrm{TNF} \alpha+\mathrm{N}=6$; $\mathrm{CON}$ $\mathrm{N}=6$ per muscle)

\section{Lipid peroxidation:}

Lipid Peroxidation Assay Kit from Oxford biosciences was used per manufacturer's instructions. Total lipid quantification was adapted from Cheng et al. 2010 [47]. (TNF $\alpha+\mathrm{N}=6$; CON N=6 per muscle)

\section{Mitochondrial Assessments}

Electron transport chain (ETC) complex activities:

ETC activities of complexes I, III, and IV were measured spectrophotometrically from whole muscle homogenate as previously described [48-50]. Briefly, complex I activity was determined by measuring the oxidation of NADH at $340 \mathrm{~nm}$ as previously described [49]. The assay mixture for complex I contained $25 \mathrm{mM}$ potassium phosphate buffer ( $\mathrm{pH} 7.2), 5 \mathrm{mM} \mathrm{MgCl} 2,2 \mathrm{mM}$ $\mathrm{KCN}, 2.5 \mathrm{mg} / \mathrm{ml} \mathrm{BSA,} 50 \mu \mathrm{M} \mathrm{NADH}, 10 \mu \mathrm{M}$ decylubiquinone, and $2 \mu \mathrm{g} / \mathrm{ml}$ antimycin A. The reaction was initiated by the addition of muscle homogenate, and enzyme activity was measured for $3 \mathrm{~min}$, with values recorded every $10 \mathrm{~s}$ after the initiation of the reaction. Complex I-specific activity was inhibited by $2 \mu \mathrm{g} / \mathrm{ml}$ rotenone. Complex III activity was determined as previously described $[48,49]$ by following the reduction of cytochrome $c$ at $550 \mathrm{~nm}$ in the presence of reduced decylubiquinone $(50 \mu \mathrm{M})$. Briefly, the assay buffer for complex III consisted of $500 \mathrm{mM}$ sucrose, 2 mM EDTA, $100 \mathrm{mM}$ Tris $\cdot \mathrm{HCl}$, (pH 7.4), $1 \mathrm{mM}$ cytochrome $c, 200 \mathrm{mM}$ KCN, 1mg/ml antimycin A, and educed decylubiquinone. Finally, complex IV activity was determined by measuring the oxidation of cytochrome $c$ at $550 \mathrm{~nm}$. Briefly, the assay mixture for complex IV consisted of $10 \mathrm{mM}$ phosphate buffer ( $\mathrm{pH} 7.4)$ and $20 \mu \mathrm{M}$ reduced cytochrome $c$. Protein 
content was determined as described previously via the bradford assay [45], and values are

expressed as activities in nmol substrate consumed per $\min ^{-1}$ per $\mathrm{mg} \operatorname{protein}^{-1}$. $(\mathrm{TNF} \alpha+\mathrm{N}=6$;

$\mathrm{CON} N=6$ per muscle)

\section{$\underline{\text { Statistics }}$}

All data are presented as means \pm SEM. To examine body mass, organ masses, muscle capillarity ex vivo muscle stimulation, and mETC activity we used a student's T-test. An alpha level at $\mathrm{P} \leq 0.05$ was selected for statistical significance.

\section{Results}

\section{Body and Muscle Mass}

Shown in Table $1, \mathrm{TNF} \alpha+$ animals were $19 \%$ smaller than $\mathrm{CON}$ animals $(\mathrm{P}<0.001)$. There was a significant decrease in muscle mass of the GA and SOL muscle of the TNF $\alpha+$ when compared to the CON. The GA was decreased by $13 \%(\mathrm{P}<0.01)$ and the SOL was decreased by $15 \%(\mathrm{P}=0.01)$ in $\mathrm{TNF} \alpha+$ compared to $\mathrm{CON}$. Lung mass was also increased $180 \%(\mathrm{P}<0.001)$. There was no difference in the PLT or heart (HRT) mass between TNF $\alpha+$ and CON (Table 1).

When muscle masses were normalized to body mass, to account for the size of the animal, none of the skeletal muscle/body masses were different between the TNF $\alpha+$ and CON. However, HRT mass/body mass was significantly increased by $10 \%(\mathrm{P}<0.01)$. Lung mass/body mass was increased by over $200 \%(\mathrm{P}<0.001)$ (Table 1$)$.

\section{Exercise capacity and muscle function.}

The TNF $\alpha+$ animals had a $120 \%$ decrease in endurance running capacity $(\mathrm{P}=0.02)$

(Figure 1), but there was no difference in in vivo muscle fatigue between the TNF $\alpha+$ and CON animals (Figure 2). 


\section{TNF $\alpha$ serum and muscle levels}

TNF $\alpha$ serum levels were increased by $299 \%$ in the TNF $\alpha+$ animals $(\mathrm{P} \leq 0.001)$ (Figure 3$)$. There was no difference in TNF $\alpha$ levels of the SOL, PLT, or GA muscles between the TNF $\alpha+$ and the CON (Table 2).

\section{Skeletal muscle capillarity}

There was a $16 \%$ decrease in capillary-to-fiber ratio $(\mathrm{C}: \mathrm{F})$ in the SOL muscle of the $\mathrm{TNF} \alpha+$ animals when compared to the $\mathrm{CON}(\mathrm{P}<0.02)$ (Figure 3$)$. There was no change in the PLT capillarity (Figure 4). There was also no difference in either of the muscles in capillary density (CD) or fiber cross sectional area (FCSA) (Figures $3 \& 4$ )

\section{Angiogenic protein content}

There was no difference in TSP-1 in either of the muscles tested (SOL, PLT) (Figures 3 and 4). There was also no difference in VEGF levels in either of the muscles tested (Figures 3 and 4).

An angiogenic protein array was used to screen a wide set of angiogenic regulators that could be altered in the TNF $\alpha+$. These data revealed that only TSP-2 was significantly increased (by $87 \%, \mathrm{p}<0.05$ ) (Figure 5). Despite a similar trend seen in other factors, such as keratinocyte growth factor $(+158 \%, \mathrm{P}=0.06)$, matrix metalloproteinase $9(+182 \%, \mathrm{P}=0.07)$, and coagulation factor III (+88\%, P=0.06), only TSP-2 resulted in a statistically significant outcome (Figures 5).

\section{Mitochondrial ETC Activity}

There was a significant $170 \%$ increase in complex III activity in the SOL of the TNF $\alpha+$ mice when compared to the $\mathrm{CON}(\mathrm{P}<0.01)$ (Table 3). There was no difference in the complex activities of either complex I or IV in the SOL. 
In the PLT, there was also a significant $74 \%$ increase in complex IV activity $(\mathrm{P}<0.05)$ (Table 3). However there was no difference in any of the activities of the other complexes.

\section{Reactive oxygen species (ROS) assessment}

DCF analysis revealed that there was no significant difference in gastrocnemius (GA) ROS production between the TNF $\alpha+$ and $\mathrm{CON}$ animal (Figure 6). The lipid peroxidation assay revealed that there was also no increase or decrease in lipid oxidation in the SOL or PLT of the $\mathrm{TNF} \alpha+$ compared to the CON. This remained true when corrected for both total lipid and total protein content (Figure 7).

\section{Discussion}

The main finding of this study was that there was a significant decrease in soleus muscle capillarity of mice with increased levels of systemic circulating TNF $\alpha$ that is associated with an elevated expression of TSP-2, but not TSP-1. This suggests that TNF $\alpha$ could play a role in skeletal muscle dysfunction that may be mediated by TSP-2.

\section{Increased systemic TNF $\alpha$ decreases capillarity}

Angiogenesis, the process of vessel growth from the existing vascular network, plays an essential role in meeting the metabolic demands of skeletal muscle. It is not surprising that capillary regression is associated with decreased exercise tolerance in several pathological conditions $[2,13,14,16,18,51-54]$, but the molecular mediators responsible for capillary regression in many of these conditions are still unknown. In this study, we showed that a TNF $\alpha$ overexpressing mouse model resulted in a $\sim 300 \%$ increase in circulating TNF $\alpha$ compared control mice. A finding similar to that previously reported in this $\mathrm{TNF} \alpha+$ mouse model $[12,55]$. Despite 
a significant decrease in SOL capillarity in $\mathrm{TNF} \alpha+$ mice, we found no change in capillarity of the PLT muscle. While a full explanation for this difference is not immediately evident, it has been shown previously in this same model of TNF $\alpha$ overexpression that the SOL muscle function is more greatly affected by TNF $\alpha$ when compared to less oxidative muscles [12]. Those data, and that of our manuscript, may seem to suggest that there is a fiber-type specific response to chronic systemic inflammation, as the SOL is composed of primary slow MHC fibers, while the PLT is primarily fast MHC fibers. The reasons for this fiber type specific response also remain unknown. However, in human diseases such as COPD, there is decreased overall aerobic capacity, which coincides with similar fiber type specific changes. Indeed, a reduction in the number of type I fibers in human quadriceps muscle is correlated with a decrease in overall exercise capacity more strongly in those with COPD than healthy controls [15]. Also, in previous work with this mouse model, it was shown that there was a shift in muscle fiber type composition in the SOL towards a faster fiber phenotype [12]. Further work will be needed to tease out this fiber specific response.

\section{Increased systemic inflammation leads to an increase in TSP-2}

Our protein analysis revealed a trend for an overall greater increases in angiogenic regulatory proteins in $\mathrm{TNFa}+$ compared to controls, however of the over 50 proteins examined only TSP- 2 was significantly altered in the TNF $\alpha+$. TSP- 2 was found to be increased by over $80 \%$ compared to CON. Surprisingly, we saw no change in TSP-1. While the function and expression of TSP-1 has been examined in skeletal muscle [23, 25, 52], the role of TSP-2 in skeletal muscle is relatively unknown. Our finding suggests that TSP-2 could be playing a role in skeletal muscle capillary regression that has not been previously recognized. Indeed, TSP-2 is 
closely related to TSP-1 in both structure and function, and has been shown to decrease capillarity in tumor models [56, 57]. TSP-2 is found to be important in development $[29,33,58]$ and in connective tissue structure and cardiac tissue healing [58-61]. TSP-2 also shares many of the common motifs of TSP-1 and has been shown to function through many of the same receptors as TSP-1 $[29,32,33,57,60]$. Collectively, we believe these data suggest that TSP-2 may play a role in inflammatory induced skeletal muscle capillary regression.

\section{In vivo muscle stimulation, exercise capacity, and mitochondrial function}

Unlike previous studies which have used invasive stimulation models [11, 12], we chose in vivo muscle stimulation because it is minimally invasive and allows us to keep all of the vasculature and connective tissue intact, and therefore more closely reflects muscle activity in a conscious exercising animal. Using this paradigm we did not find greater muscle fatigue in the $\mathrm{TNF} \alpha+$ mice. It should be noted, this in vivo stimulation paradigm reflects the fatigue of the whole hindlimb tricep complex and therefore can not differentiate between different muscle types (e.g. oxidative vs. glycolytic). Given that we only found capillarity to be altered in the SOL, it is perhaps not surprising that there is no change in in vivo muscle function, as the SOL makes up the smallest contribution to overall force production in the hindlimb.

Despite no evidence of muscle contractile dysfunction, $\mathrm{TNF} \alpha+$ mice exhibited a significant decrease in whole body exercise capacity (i.e. running endurance). This is most likely a consequence of the central lung pathology found in this model, which is similar to that observed in COPD [39]. Our data support the recent finding of decreased maximal running capacity observed in male TNF $\alpha+$ mice [12]. Surprisingly, however, the previous study found no effect on exercise capacity in female mice [12]. Our study included both males and females, but 
we did not find significant difference between the sexes. At present, the reason for this discrepancy between these studies is unknown, but will required further investigation.

It has been shown recently that PGC- $1 \alpha$ expression and citrate synthase activity is decreased in the SOL of TNF $\alpha+$ mice [12]. Based on this observation, we expected that mitochondrial electron transport chain enzyme activity might be reduced. To the contrary, we found a $170 \%$ increase in complex III activity in the SOL and a $74 \%$ increase in complex IV activity in the PLT of the TNF $\alpha+$, with a similar trend (albeit not statistically significant) for increased mitochondrial ETC activity across almost all of the complexes for both the SOL and PLT muscles. This may be a mechanism by which skeletal muscle mitochondria are compensating for fewer mitochondria (i.e. decrease in CS activity). Further work with electron microscopy will need to be conducted to elucidate what is happening with mitochondrial density.

To further evaluate mitochondria function in the skeletal muscle of TNF $\alpha+$ mice, we also measured total ROS content and lipid oxidation (LPO). We saw no change in LPO and/or any alterations in ROS production in the skeletal muscle. Both measures are non-specific measurements of overall ROS activity and suggest that the degree of muscle capillarity loss seen in the SOL muscle is not likely linked to a ROS mediated event. Given the prior evidence of reduced PGC1- $\alpha$ and citrate synthase activity in this mouse model [12], and our measurements showing no changes in ROS, it seems likely that $\mathrm{TNF} \alpha+$ mice simply have less mitochondria, rather than dysfunctional mitochondria per se. This is also supported by the finding that mitochondrial ETC data were not decreased in TNF $\alpha+$ mice, but rather, in some cases actually increased. 


\section{Summary}

In summary, we show that increased systemic TNF $\alpha$ decreases skeletal muscle capillarity. This rarefaction appears to be specific to oxidative skeletal muscle, and may be linked to TSP-2 expression. While endurance exercise capacity was reduced, muscle mitochondrial electron transport chain activity was increased and muscle contractile function was not changed, suggesting the exercise intolerance is not at the mitochondrial or muscle contractile level. While the mechanisms that connects capillary rarefaction and systemic inflammation still remain obfuscated, our data suggest that chronic elevation of TNF $\alpha$ may act by stimulating TSP- 2 expression and that this TNF $\alpha$ TSP-2 axis may provide a fruitful therapeutic target aimed at combating skeletal muscle co-morbidities. 


\section{Acknowledgements}

The authors would like to acknowledge Janelle Stricker and Nicole Zachwieja for their contribution to the data in this manuscript.

\section{Conflict of interest}

The authors report no conflict of interest 
Citation

1. Danesh, J., et al., Low grade inflammation and coronary heart disease: prospective study and updated meta-analyses. BMJ, 2000. 321(7255): p. 199-204.

2. Gosker, H.R., et al., Skeletal muscle dysfunction in chronic obstructive pulmonary disease and chronic heart failure: underlying mechanisms and therapy perspectives. The American Journal of Clinical Nutrition, 2000. 71(5): p. 1033-1047.

3. $\mathrm{Xu}, \mathrm{H}$., et al., Chronic inflammation in fat plays a crucial role in the development of obesity-related insulin resistance. The Journal of Clinical Investigation, 2003. 112(12): p. 1821-1830.

4. Duncan, B.B., et al., Low-Grade Systemic Inflammation and the Development of Type 2 Diabetes. Diabetes, 2003. 52(7): p. 1799-1805.

5. Kapadia, S.R., et al., Elevated circulating levels of serum tumor necrosis factor-alpha in patients with hemodynamically significant pressure and volume overload. Journal of the American College of Cardiology, 2000. 36(1): p. 208-212.

6. Feldman, A.M., et al., The role of tumor necrosis factor in the pathophysiology of heart failure. Journal of the American College of Cardiology, 2000. 35(3): p. 537-544.

7. Di Francia, M., et al., Tumor necrosis factor-alpha levels and weight loss in chronic obstructive pulmonary disease. Am J Respir Crit Care Med, 1994. 150(5 Pt 1): p. 1453-5.

8. Dandona, P., A. Aljada, and A. Bandyopadhyay, Inflammation: the link between insulin resistance, obesity and diabetes. Trends in immunology, 2004. 25(1): p. 4-7.

9. Yamakawa, I., et al., Inactivation of TNF- $\alpha$ ameliorates diabetic neuropathy in mice. American Journal of Physiology - Endocrinology And Metabolism, 2011. 301(5): p. E844-E852.

10. Anker, S.D. and S. von Haehling, Inflammatory mediators in chronic heart failure: an overview. Heart, 2004. 90(4): p. 464-470.

11. Zuo, L., L. Nogueira, and M.C. Hogan, Effect of pulmonary TNF-alpha overexpression on mouse isolated skeletal muscle function. Am J Physiol Regul Integr Comp Physiol, 2011. 301(4): p. R1025-31.

12. Tang, K., et al., Impaired exercise capacity and skeletal muscle function in a mouse model of pulmonary inflammation. J Appl Physiol, 2013. 114(9).

13. Kivela, R., et al., Exercise-induced expression of angiogenic growth factors in skeletal muscle and in capillaries of healthy and diabetic mice. Cardiovascular Diabetology, 2008. 7(1): p. 13.

14. Kivelä, R., et al., Effects of experimental type 1 diabetes and exercise training on angiogenic gene expression and capillarization in skeletal muscle. The FASEB journal, 2006. 20(9): p. 1570-1572.

15. Allaire, J., et al., Peripheral muscle endurance and the oxidative profile of the quadriceps in patients with COPD. Thorax, 2004. 59(8): p. 673-678.

16. Jobin, J., et al., Chronic obstructive pulmonary disease: capillarity and fiber-type characteristics of skeletal muscle. J Cardiopulm Rehabil, 1998. 18(6): p. 432-7.

17. Wouters, E.F.M., E.C. Creutzberg, and A.M.W.J. Schols, SYstemic effects in copd*. CHEST Journal, 2002. 121(5_suppl): p. 127S-130S.

18. Duscha, B.D., et al., Capillary density of skeletal muscle: A contributing mechanism for exercise intolerance in class II-III chronic heart failure independent of other peripheral alterations. Journal of the American College of Cardiology, 1999. 33(7): p. 1956-1963. 
19. Folkman, J., Angiogenesis. Annu. Rev. Med., 2006. 57: p. 1-18.

20. Hanahan, D. and J. Folkman, Patterns and Emerging Mechanisms of the Angiogenic Switch during Tumorigenesis. Cell, 1996. 86(3): p. 353-364.

21. Mark Olfert, I. and O. Birot, Importance of anti angiogenic factors in the regulation of skeletal muscle angiogenesis. Microcirculation, 2011.

22. Audet, G.N., et al., Chronic delivery of a thrombospondin-1 mimetic decreases skeletal muscle capillarity in mice. PLoS One, 2013. 8(2): p. e55953.

23. Audet, G.N., et al., Expression of angiogenic regulators and skeletal muscle capillarity in selectively bred high aerobic capacity mice. Experimental physiology, 2011. 96(11): p. 1138-1150.

24. Malek, M.H. and I.M. Olfert, Global deletion of thrombospondin 1 increases cardiac and skeletal muscle capillarity and exercise capacity in mice. Experimental physiology, 2009. 94(6): p. 749-760.

25. Roudier, E., et al., Angio-adaptation in unloaded skeletal muscle: new insights into an early and muscle type-specific dynamic process. The Journal of Physiology, 2010. 588(22): p. 4579.

26. Krady, M.M., et al., Thrombospondin-2 modulates extracellular matrix remodeling during physiological angiogenesis. The American Journal of Pathology, 2008. 173(3): p. 879-891.

27. Gu, J.-W., et al., Tissue endostatin correlates inversely with capillary network in rat heart and skeletal muscles. Angiogenesis, 2006. 9(2): p. 93-99.

28. Brixius, K., et al., Long-term endurance exercise decreases antiangiogenic endostatin signalling in overweight men aged 50-60 years. British journal of sports medicine, 2008. 42(2): p. 126-129.

29. Lawler, J., The functions of thrombospondin-1 and-2. Current opinion in cell biology, 2000. 12(5): p. 634-640.

30. Mosher, D.F., Physiology of thrombospondin. Annual review of medicine, 1990. 41(1): p. 85-97.

31. Ren, B., et al., Regulation of tumor angiogenesis by thrombospondin-1. Biochimica et Biophysica Acta (BBA) - Reviews on Cancer, 2006. 1765(2): p. 178-188.

32. Bornstein, P., Thrombospondins function as regulators of angiogenesis. Journal of Cell Communication and Signaling, 2009. 3(3): p. 189-200.

33. Lawler, J. and M. Detmar, Tumor progression: the effects of thrombospondin-1 and -2 . The International Journal of Biochemistry \&amp; Cell Biology, 2004. 36(6): p. 10381045.

34. Chen, H., M.E. Herndon, and J. Lawler, The cell biology of thrombospondin-1. Matrix Biology, 2000. 19(7): p. 597-614.

35. Salajegheh, M., et al., Upregulation of thrombospondin-1(TSP-1) and its binding partners, CD36 and CD47, in sporadic inclusion body myositis. Journal of Neuroimmunology, 2007. 187(1-2): p. 166-174.

36. Lutz, J., et al., Increased Plasma Thrombospondin-1 (TSP-1) Levels Are Associated with the TNFa-308A Allele in Children with Juvenile Dermatomyositis. Clinical Immunology, 2002. 103(3): p. 260-263.

37. Netea, M.G., et al., Increased voluntary exercise in mice deficient for tumour necrosis factor- $\alpha$ and lymphotoxin- $\alpha$. European Journal of Clinical Investigation, 2007. 37(9): p. 737-741. 
38. Frazier, E.P., et al., Age-dependent regulation of skeletal muscle mitochondria by the thrombospondin-1 receptor CD47. Matrix Biology, 2011. 30(2): p. 154-161.

39. Fujita, M., et al., Overexpression of tumor necrosis factor-alpha produces an increase in lung volumes and pulmonary hypertension. Am J Physiol Lung Cell Mol Physiol, 2001. 280(1): p. L39-49.

40. $\quad$ Olfert, I.M., et al., Temporal thrombospondin-1 mRNA response in skeletal muscle exposed to acute and chronic exercise. Growth Factors, 2006. 24(4): p. 253-259.

41. Bruce, R.A., Exercise testing of patients with coronary heart disease. Principles and normal standards for evaluation. Ann Clin Res, 1971. 3(6): p. 323-32.

42. Mrázková, O., M. Grim, and B.M. Carlson, Enzymatic heterogeneity of the capillary bed of rat skeletal muscles. American Journal of Anatomy, 1986. 177(2): p. 141-148.

43. Lojda, Z., Studies on dipeptidyl(amino)peptidase IV (glycyl-proline naphthylamidase). Histochemistry, 1979. 59(3): p. 153-166.

44. $\quad$ PA., S., Citrate synthase. Methods Enzymol, 1969. 13: p. 3-5.

45. Bradford, M.M., A rapid and sensitive method for the quantitation of microgram quantities of protein utilizing the principle of protein-dye binding. Analytical biochemistry, 1976. 72(1): p. 248-254.

46. Best, T.M., et al., Free radical activity, antioxidant enzyme, and glutathione changes with muscle stretch injury in rabbits. Journal of Applied Physiology, 1999. 87(1): p. 74-82.

47. Cheng, Y.S., Y. Zheng, and J.S. VanderGheynst, Rapid quantitative analysis of lipids using a colorimetric method in a microplate format. Lipids, 2011. 46(1): p. 95-103.

48. Dabkowski, E.R., et al., Diabetic cardiomyopathy-associated dysfunction in spatially distinct mitochondrial subpopulations. American Journal of Physiology-Heart and Circulatory Physiology, 2009. 296(2): p. H359-H369.

49. Trounce, I.A., et al., Assessment of mitochondrial oxidative phosphorylation in patient muscle biopsies, lymphoblasts, and transmitochondrial cell lines. Methods in enzymology, 1996. 264: p. 484.

50. Rodriguez-Bies, E., et al., Muscle physiology changes induced by every other day feeding and endurance exercise in mice: effects on physical performance. PLoS One, 2010. 5(11): p. e13900.

51. Clark, C., et al., Skeletal muscle strength and endurance in patients with mild COPD and the effects of weight training. European Respiratory Journal, 2000. 15(1): p. 92-97.

52. Gouzi, F., et al., Blunted muscle angiogenic training-response in copd patients versus sedentary controls. European Respiratory Journal, 2012.

53. Man, W., et al., Skeletal muscle dysfunction in COPD: clinical and laboratory observations. Clinical Science, 2009. 117: p. 251-264.

54. Degens, H. and S.E. Alway, Control of muscle size during disuse, disease, and aging. International journal of sports medicine, 2006. 27(2): p. 94-99.

55. Langen, R.C., et al., Muscle wasting and impaired muscle regeneration in a murine model of chronic pulmonary inflammation. American journal of respiratory cell and molecular biology, 2006. 35(6): p. 689-696.

56. Nakamura, M., et al., Thrombospondin-2 inhibits tumor cell invasion through the modulation of MMP-9 and uPA in pancreatic cancer cells. Mol Med Rep, 2008. 1(3): p. 423-7. 
57. Koch, M., et al., CD36-mediated activation of endothelial cell apoptosis by an $\mathrm{N}$ terminal recombinant fragment of thrombospondin-2 inhibits breast cancer growth and metastasis in vivo. Breast Cancer Res Treat, 2011. 128(2): p. 337-46.

58. Reinecke, H., et al., Lack of thrombospondin-2 reduces fibrosis and increases vascularity around cardiac cell grafts. Cardiovasc Pathol, 2013. 22(1): p. 91-5.

59. Kyriakides, T.R., et al., Mice that lack thrombospondin 2 display connective tissue abnormalities that are associated with disordered collagen fibrillogenesis, an increased vascular density, and a bleeding diathesis. The Journal of cell biology, 1998. 140(2): p. 419-430.

60. Yang, Z., D.K. Strickland, and P. Bornstein, Extracellular Matrix Metalloproteinase 2 Levels Are Regulated by the Low Density Lipoprotein-related Scavenger Receptor and Thrombospondin 2. Journal of Biological Chemistry, 2001. 276(11): p. 8403-8408.

61. Schellings, M.W.M., et al., Thrombospondins in the heart: potential functions in cardiac remodeling. Journal of Cell Communication and Signaling, 2009. 3(3): p. 201-213. 
Table 1: Muscle, heart, and lung masses

\begin{tabular}{|l|c|c|}
\hline & WT & TNFa+ \\
\hline BM (g) & $31.9 \pm 1.5$ & $25.9 \pm 0.8^{*}$ \\
\hline GA (mg) & $141 \pm 3$ & $122 \pm 4^{*}$ \\
\hline PLT (mg) & $18 \pm 0.7$ & $19 \pm 0.3$ \\
\hline SOL (mg) & $9 \pm 0.4$ & $8 \pm 0.3^{*}$ \\
\hline Heart (mg) & $130 \pm 3$ & $135 \pm 3$ \\
\hline Lungs (mg) & $188 \pm 6$ & $530 \pm 17^{*}$ \\
\hline GA/BM (mg $\cdot \mathbf{g - 1})$ & $4.5 \pm 0.1$ & $4.7 \pm 0.6$ \\
\hline PLT/BM (mg・g-1) & $0.6 \pm 0.02$ & $0.8 \pm 0.1$ \\
\hline SOL/BM (mg $\cdot \mathbf{g - 1})$ & $0.3 \pm 0.01$ & $0.3 \pm 0.01$ \\
\hline Heart/BM (mg・g-1) & $5.0 \pm 0.1$ & $5.5 \pm 0.1 *$ \\
\hline Lungs/BM (mg・g-1) & $7.3 \pm 0.1$ & $21.9 \pm 0.7^{*}$ \\
\hline
\end{tabular}

All values \pm SE. * $P \leq 0.05$ between groups 
Table 2: TNF $\alpha$ Levels in Serum and Muscle

\begin{tabular}{|c|c|c|}
\cline { 2 - 3 } \multicolumn{1}{c|}{} & WT & TNFa+ \\
\hline $\begin{array}{c}\text { Serum } \\
(\mathbf{p g} / \mathbf{m L})\end{array}$ & $87 \pm 17$ & $347 \pm 55 *$ \\
\hline $\begin{array}{c}\mathbf{G A} \\
\text { (pg/mg) }\end{array}$ & $26 \pm 2$ & $22 \pm 2$ \\
\hline $\begin{array}{c}\mathbf{P L T} \\
\mathbf{( p g / m g )}\end{array}$ & $151 \pm 22$ & $129 \pm 35$ \\
\hline $\begin{array}{c}\text { SOL } \\
\text { (pg/mg) }\end{array}$ & $40 \pm 11$ & $37 \pm 10$ \\
\hline
\end{tabular}

All Values \pm SE. * $\mathrm{P} \leq 0.05$ from $\mathrm{WT}$ 
Table 3: Mitochondrial Electron Transport Chain (mETC) Activity

\begin{tabular}{|c|c|c|c|}
\hline & \multicolumn{3}{|c|}{ Soleus } \\
\hline & Complex I & Complex III & Complex IV \\
\hline WT & $7.3+/-1.6$ & $3.1+/-0.5$ & $12.1+/-1.6$ \\
\hline TNF $\alpha+$ & $9.36+/-1.7$ & $8.4+/-1.6 *$ & $19.9+/-4.9$ \\
\hline & \multicolumn{3}{|c|}{ Plantaris } \\
\hline & Complex I & Complex III & Complex IV \\
\hline WT & $4.2+/-0.4$ & $1.6+/-0.2$ & $2.7+/-0.4$ \\
\hline TNF $\alpha+$ & $3.9+/-0.6$ & $2.4+/-0.3$ & $4.6+/-0.7 *$ \\
\hline
\end{tabular}

All values $\mathrm{nmol}$ of $\mathrm{NADH} / \mathrm{min} / \mathrm{mg}$ total protein. $*=\mathrm{P} \leq 0.05$ from WT 


\section{Figure Legends}

Figure 1: Endurance running capacity is significantly decreased in $\mathrm{TNF} \alpha+$ mice. There was a $120 \%$ decrease in running capacity in the TNF $\alpha+$ group compared to WT. Mice ran at $20 \mathrm{~m} / \mathrm{min}$ at a $10^{\circ}$ angle for as long as possible. Values $\pm \mathrm{SE}$.

Figure 2: In vivo muscle fatigue is unchanged in TNF $\alpha+$ mice. The whole triceps surae complex was subjected to a fatigue protocol $(12 \mathrm{v}, 40 \mathrm{hz}, 333 \mathrm{~ms}$, every second for 180 seconds ) via stimulation of the tibial nerve. A) Absolute values. B) $\%$ Change from first time point Values \pm SE.

Figure 3: Soleus capillarity is decreased in TNF $\alpha+$ animals, while TSP-1 and VEGF remain unchanged. A) There was a $16 \%$ reduction in capillary-to-fiber ratio (C:F) in the SOL. B) \& C) Despite a trend $(\mathrm{P}=0.01)$ there was no change in capillary density $(\mathrm{CD})$, or fiber cross sectional area (FCSA). D) \& E) There was no change in VEGF measured via ELISA or TSP-1 levels measured via western blot. F) Representative TSP-1 blot. Values \pm SE.

Figure 4: Plantaris capillarity, TSP-1, and VEGF remain unchanged in TNF $\alpha+$ mice. A-C) There was no change in capillary-to-fiber ratio (C:F), capillary density (CD) or fiber cross sectional area (FCSA) in the PLT. D) \& E) There was no change in VEGF measured via ELISA or TSP1 levels measured via western blot. F) Representative TSP-1 blot. Values \pm SE. 
Figure 5: Angiogenic array reveals of 53 proteins measured, only TSP-2 is changed in TNF $\alpha+$ mice. 100ug of total protein was analyzed for 53 different angiogenic proteins. The only protein that was statistically changed in the TNF $\alpha+$ was TSP-2 (82\% increased, $\mathrm{P}=0.02)$. Values $\pm \mathrm{SE}$.

Figure 6: No change in overall ROS content in TNF $\alpha+$ mice. The DCF assay revealed no change in overall ROS content in the SOL or PLT of TNF $\alpha+$ animals. A) SOL, B) PLT. Values \pm SE.

Figure 7: No change in lipid oxidation in TNF $\alpha+$ mice. There was no change in lipid oxidation as measured by the LPO assay in the GA of TNF $\alpha+$ mice. A) Absorbance/total lipid content B) Absorbance/total protein content. Values \pm SE. 
Figure 1

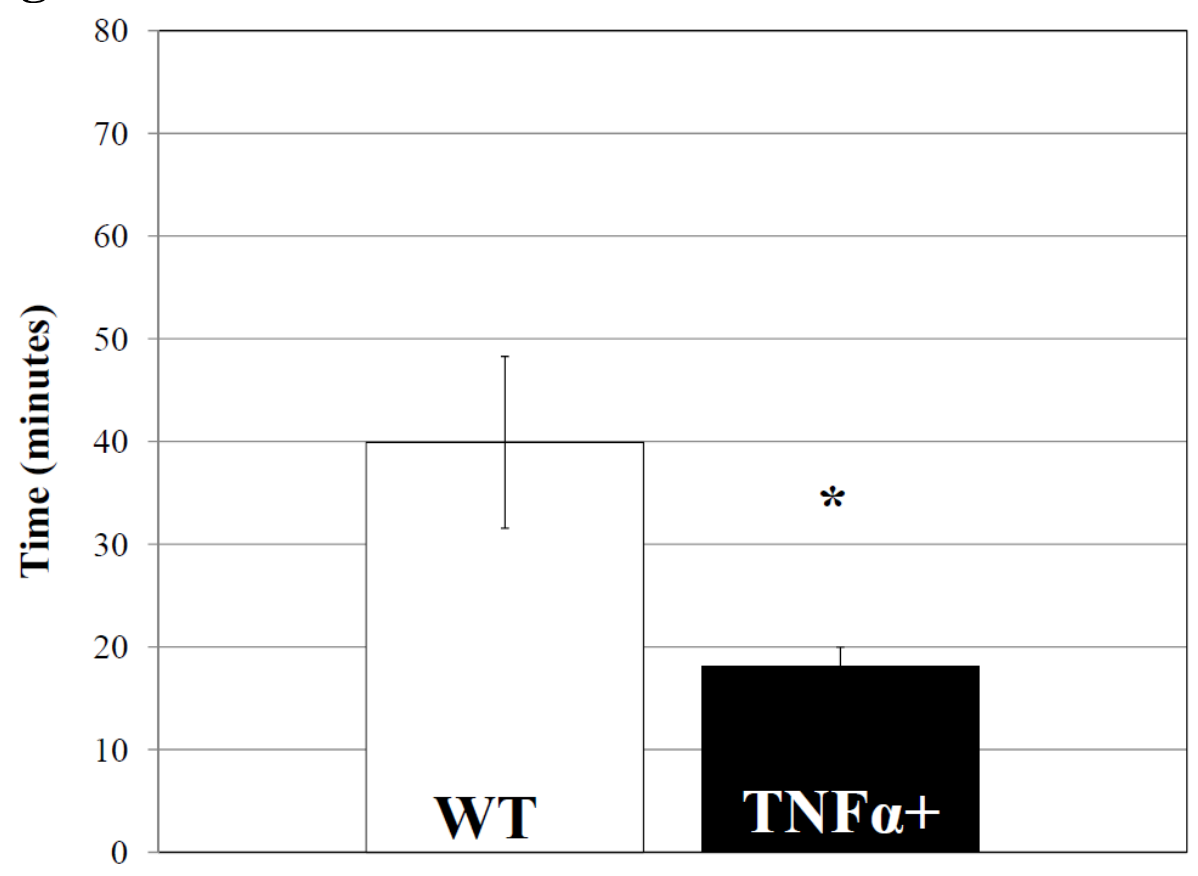


Figure 2
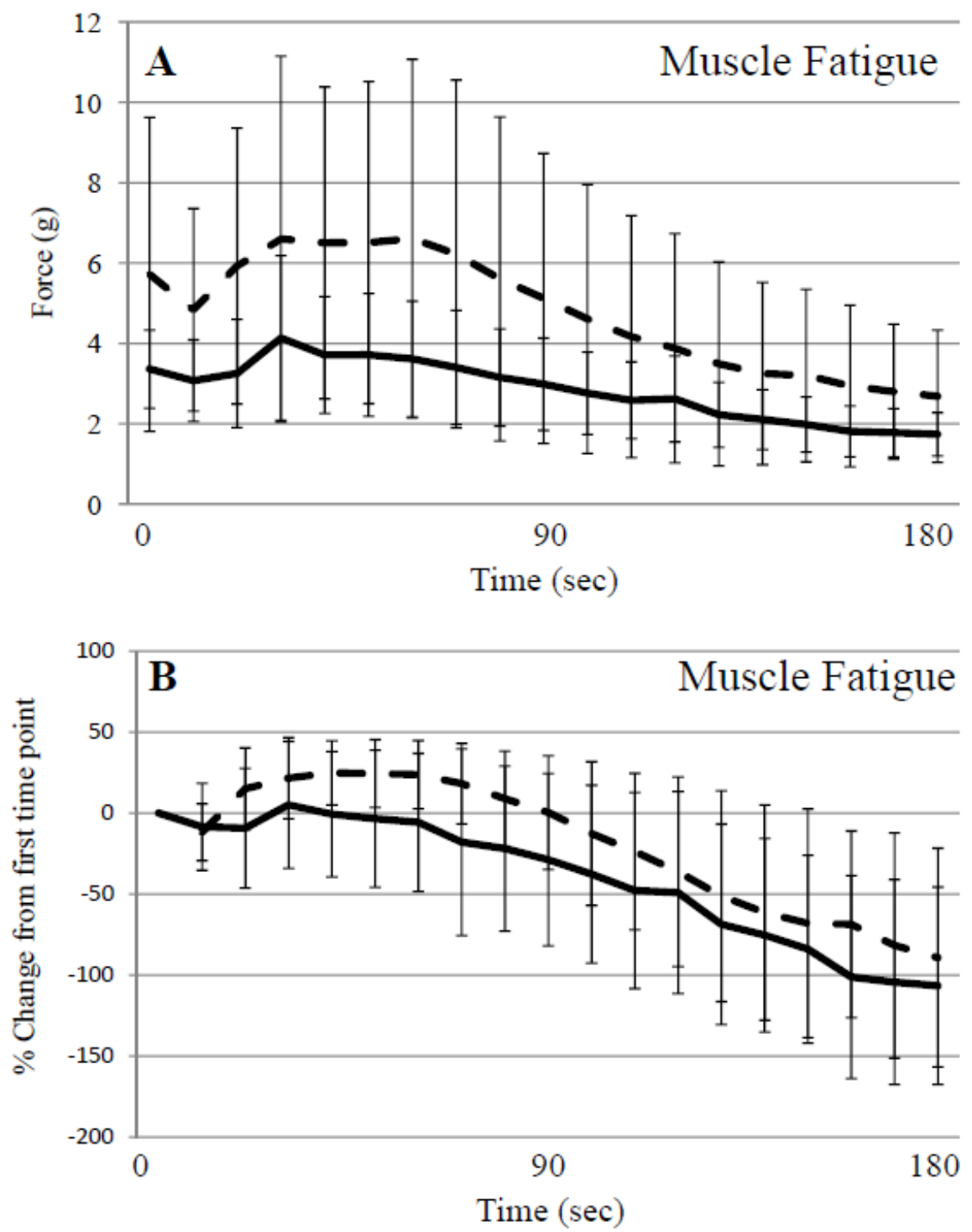
Figure 3

\section{SOL}
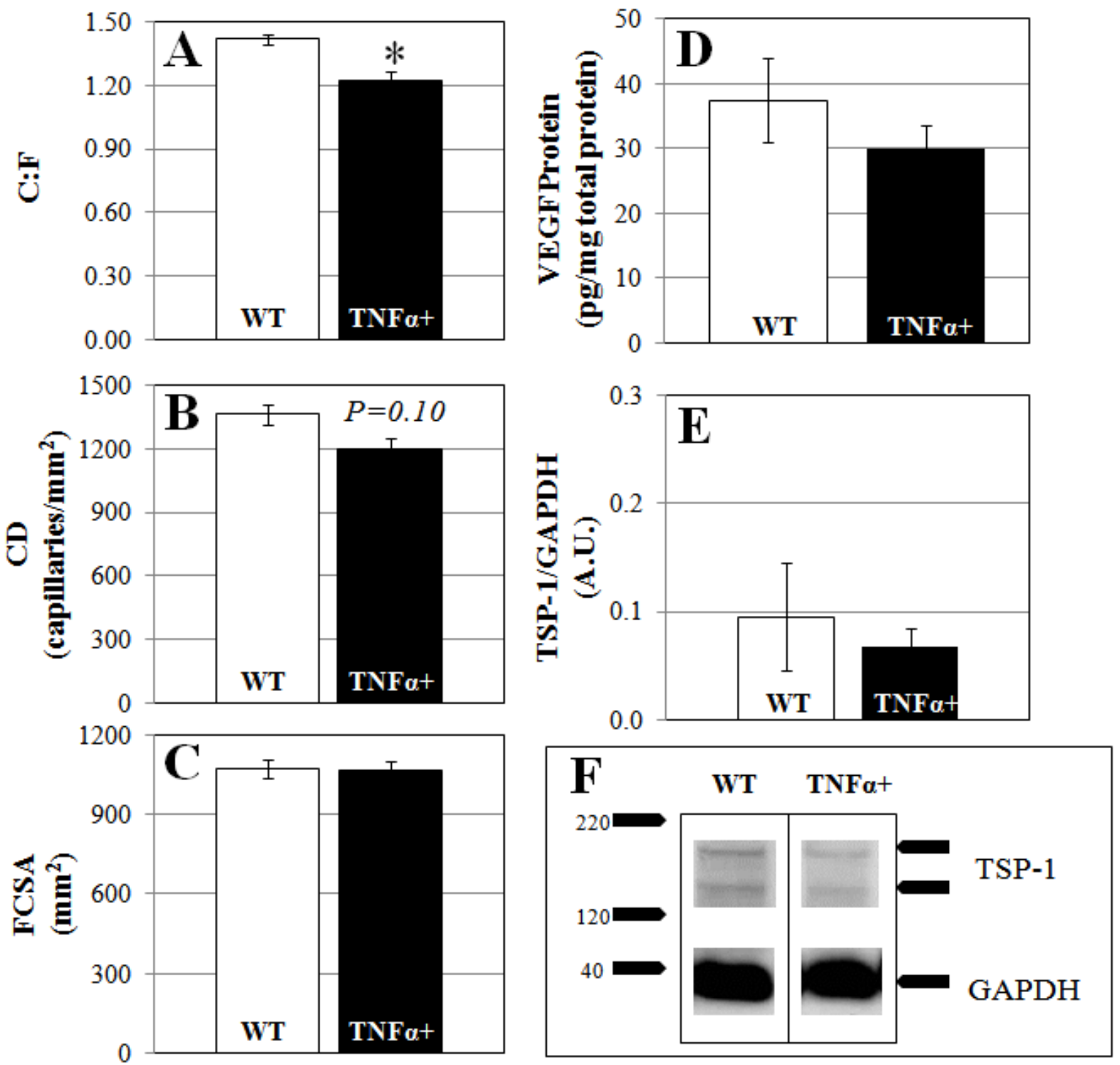
Figure 4

\section{PLT}
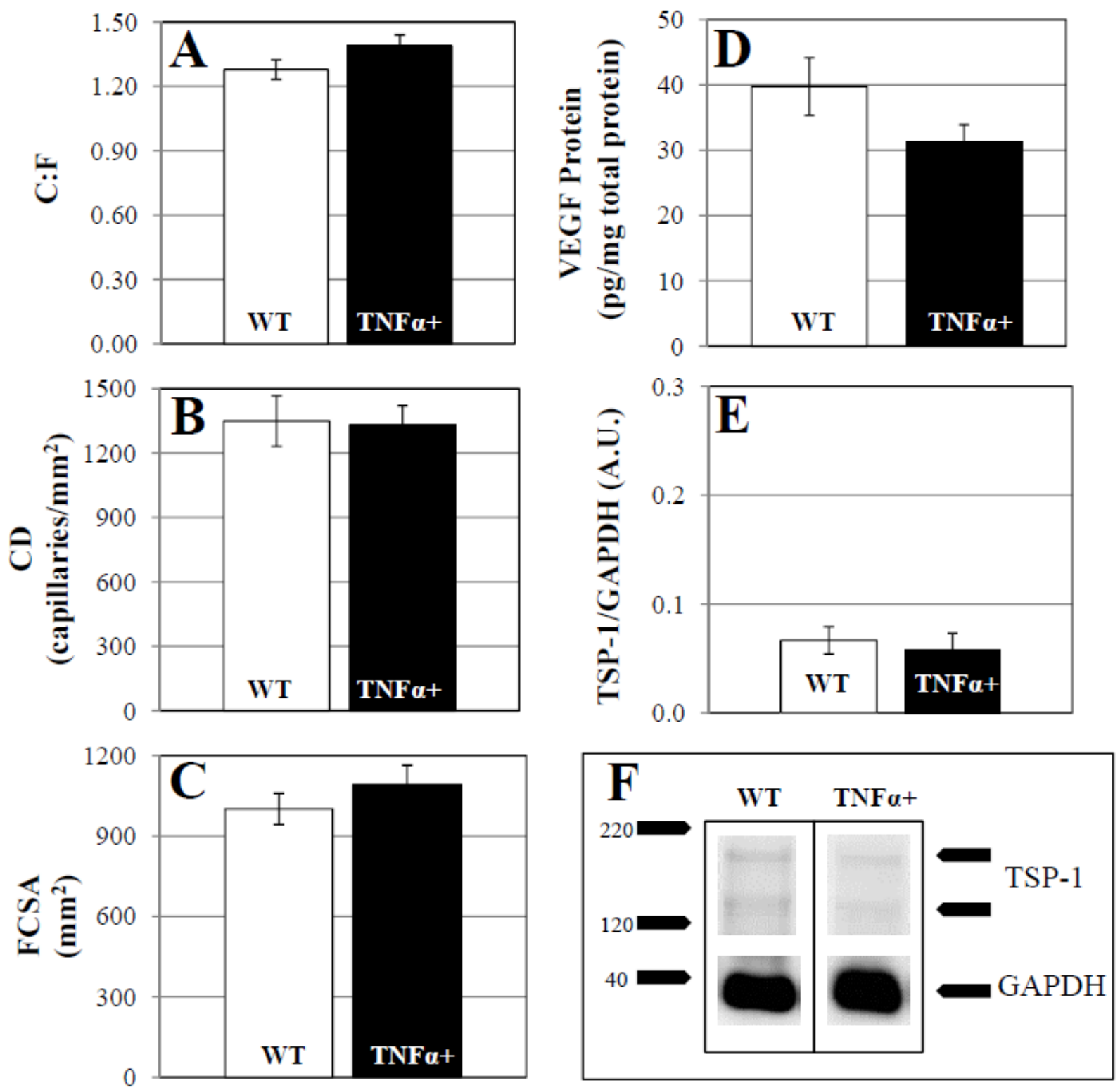


\section{Figure 5}

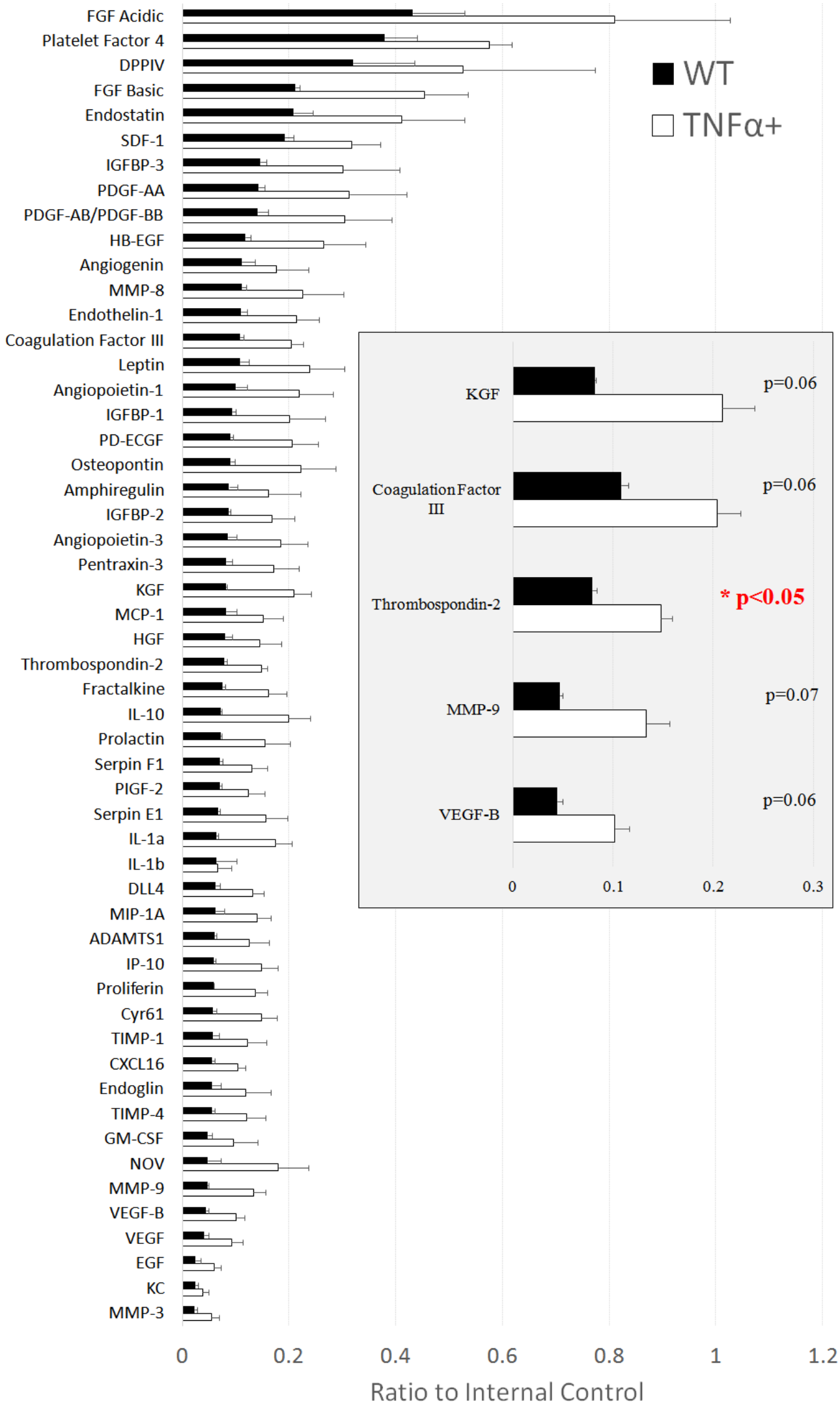


Figure 6
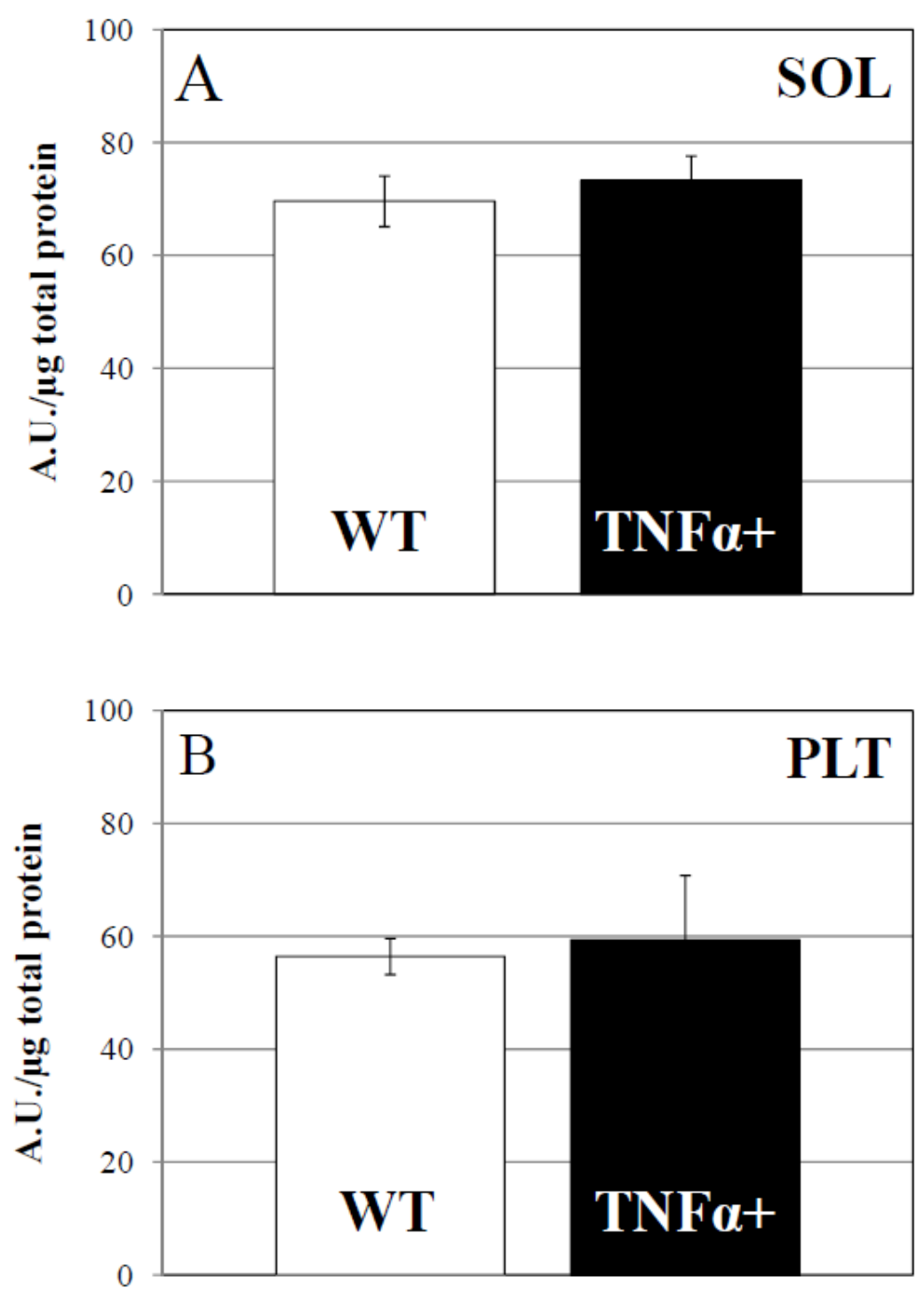
Figure 7
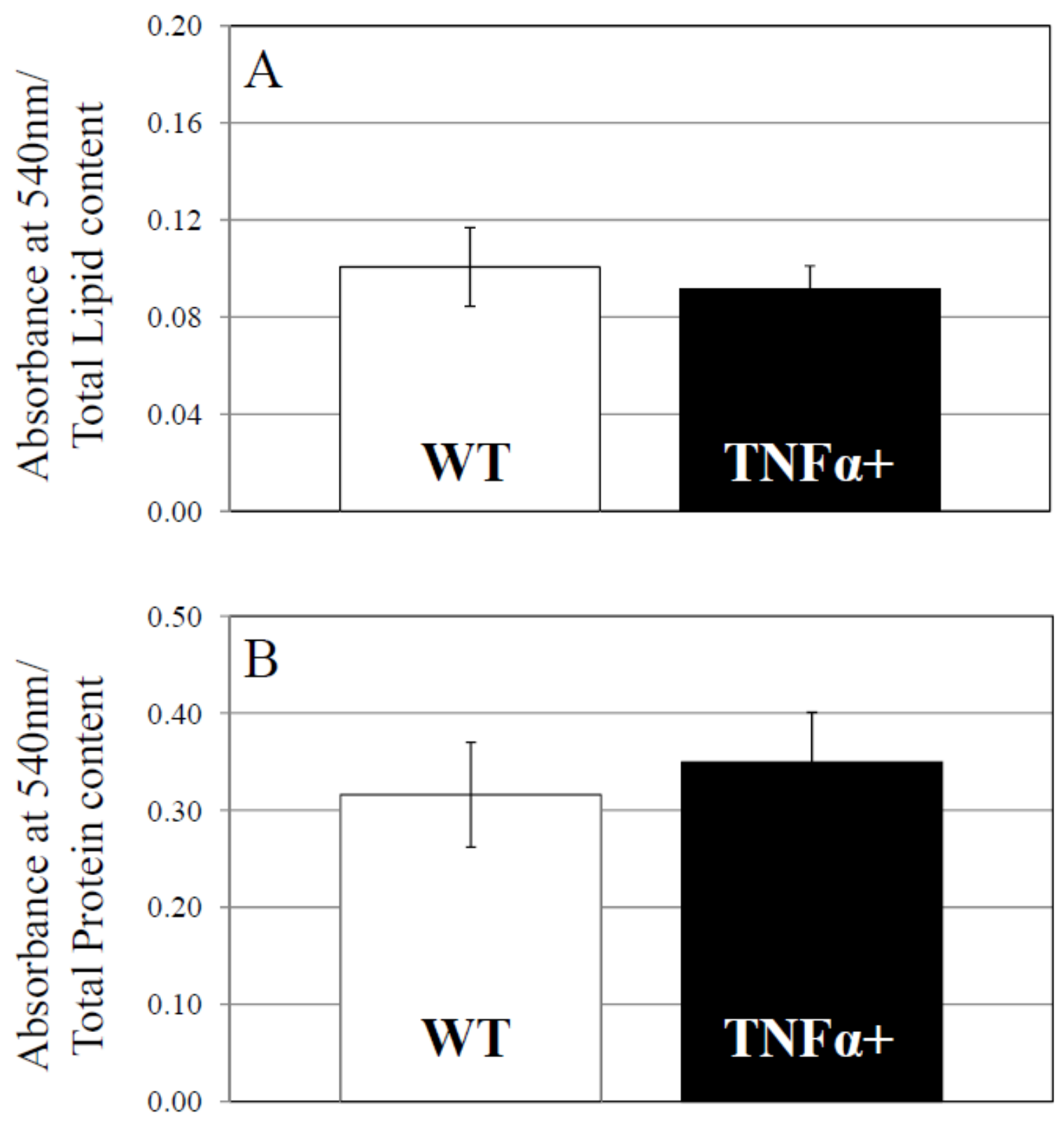
This page intentionally left blank 


\section{Chapter 6: General Discussion}




\section{Thrombospondin-1 is critical in physiological angiogenesis, but it does not play a role in inflammatory induced capillary regression}

While TSP-1 was discovered almost half a century ago, it wasn't until 1990 that it was shown in vivo to be a potent anti-angiogenic protein [1], and it wasn't until some years later that it was suggested to be important in skeletal muscle $[2,3]$. Since then, the body of work supporting the role of TSP-1 in skeletal muscle has grown, but a void continues to exist and critical questions remain unanswered. While TSP-1 has been shown to be important in cancer, only a limited amount of work has been performed on its role in physiological skeletal muscle angiogenesis [2-5], and an even smaller body of work has been published on its potential role in pathological skeletal muscle angiogenesis [5-7].

In chapter 3, we showed that TSP-1 is critical in controlling physiological skeletal muscle angiogenesis. Through the chronic administration of a TSP-1 mimetic, we showed that the actions of TSP-1 result in a decrease of skeletal muscle capillarity across three distinct muscle types (oxidative, glycolytic, and mixed muscles). Further, because the mimetic used in this study is a CD36 agonist, this provides evidence that TSP-1's anti-angiogenic properties in skeletal muscle involve a CD36 mediated mechanism. Indeed, this is consistent with previous work showing the binding of TSP-1 to CD36 is anti-proliferative, anti-angiogenic, and pro-apoptotic [8-12]. Because we showed this same response in skeletal muscle under physiological conditions, there appears to be a putative role for TSP-1/CD36 in decreasing vascularity across multiple tissues. In combination with the findings that TSP-1 KO's having increased capillarity[3], these data provide clear evidence thatTSP-1 is a physiological regulator of skeletal muscle vascularity. Indeed, further work from our lab supports the importance of TSP-1 by showing there is a reduction in TSP-1 protein in response to training during the time that 
capillary adaptation is occurring (figure 1 of the appendix). This supports previous work with mRNA [2], and suggests that TSP-1 may serve in the course of training as a vascular regulatory protein, furthering TSP-1's role beyond the basal control of capillarity seen in chapter 3. These data then sets the ground work to study aberrant skeletal muscle angiogenesis and /or capillary rarefaction in chronic disease conditions.

In chapter 5, we pursued one of the mechanisms that is potentially important in skeletal muscle co-morbidities of chronic disease. Chronic systemic inflammation has been suggested to play a crucial role in skeletal muscle capillary rarefaction associated with chronic conditions. Chronic sytemic inflammation has been shown to be important in a host of syndromes and diseases [13-16], where it has been suggested to be linked to both co-morbidities and overall health outcomes [17]. The chronic-inflammatory model we chose for chapter 5 is a lung specific overexpressor of TNF $\alpha$. TNF $\alpha$ is an important inflammatory cytokine that is linked to many of the secondary effects of chronic disease $[13-16,18,19]$. The lung specific overexpression in this model results in a spill over systemically, resulting in higher circulating TNFa. This mouse line has been suggested to be representative of chronic lung disease in humans, such as chronic obstructive pulmonary disease (COPD) [20-22]. COPD is also associated (by an unknown mechanism) with skeletal muscle dysfunction [5, 23-29], and it has been suggested this could be due in part to alterations in skeletal muscle capillarity $[5,24,30]$.

The main finding of our studies in chapter 5 was that there was a reduction in skeletal muscle capillarity in association with increased systemic TNF $\alpha$. The reduction in capillarity was only found in the soleus, and not the plantaris. Although we cannot establish a direct cause-andeffect relationship between $\mathrm{TNF} \alpha$ and muscle capillarity based solely on these data, there are numerous studies that find elevated circulating $\mathrm{TNF} \alpha$ in concert with reduced skeletal muscle 
capillarity [14, 21, 23-25, 28, 31-33]. This work, in combination with our data, points to a causative role of TNF $\alpha$ in the reduction of skeletal muscle capillarity, and establishes the importance of TNF $\alpha$ in chronic inflammation induced muscle dysfunction.

We hypothesized a TNF $\alpha$-TSP-1 axis mediated mechanism for capillary rarefaction based on in vitro work pointing to a skeletal muscle specific TNF $\alpha$-TSP-1 connection [34]. Surprisingly, we found that skeletal muscle TSP-1 protein levels were unchanged in vivo with elevated systemic TNF $\alpha$. This was true for both muscles where capillarity was changed (soleus) and those that remained unchanged (plantaris). This led us to conclude that, at least in the context of TNF $\alpha$ systemic overexpression, TSP-1 is not the key player linking chronic systemic inflammation to a loss in capillarity.

However, given the apparent importance of TPS-1 in physiological angiogenesis, as shown in chapter 3, we also examined TSP-1's expression in three other rodent models of systemic inflammation- two obesity models and a cigarette smoke exposure model. Previously, it has been shown that all of these have increased general levels of systemic inflammation, and increased levels of TNF $\alpha$ specifically $[35,36]$. Supporting the work in chapter 5 , none of these models showed any change in skeletal muscle TPS-1, despite clear previous evidence of decreased capillarity [33, 37].

Given the lack of change in skeletal muscle TSP-1 in all of these models, we can only conclude that TSP-1 does not play an important role in TNF $\alpha$ driven, systemic inflammation induced, pathological capillary regression. This is despite its apparent critical role in physiological angiogenesis (as seen in Chapter 3). Supporting this notion, in a recent publication looking at what controls skeletal muscle angiogenesis in human COPD patients, basal levels of TSP-1 are unchanged between COPD patients and sedentary controls, yet the COPD individuals 
have decreased capillarity [5]. However, with six weeks of exercise training TSP-1 levels drop significantly, which was not shown in the control group. This lack of a baseline difference, but a retained response to an exercise stimulus, further suggests that TSP-1 is not important in the pathological loss of capillaries, but is important in the physiological response to an angiogenic stimulus.

\section{The Thrombospondins play an important role in the angiogenic balance}

An altered TSP-1 to VEGF ratio is thought to be important in the control of skeletal muscle capillarity $[6,38]$. In chapter 3 we found a decrease in VEGF with chronically elevated levels of TSP-1, supporting these previous findings. These data also support previous in vitro studies suggesting that TSP-1 may be binding and sequestering VEGF - resulting eventually in internalization and biological inactivation [39-43]. Explanations for the reduction in VEGF in chapter 3 may also involve suppression of VEGF production and/or VEGF signaling. For example, there is a growing body of evidence showing that internalization of the VEGF/VEGFR2 complex by endocytosis results in downstream activation of several different signaling pathways [44]. There is also evidence that both CD36 and CD47 can associate with VEGFR-2, and when TSP-1 is present this association can prevent the VEGF ligand binding to its receptor, as well as inducing receptor dephosphorylation and preventing dimerization [45-48]. In this way, TSP-1 could prevent activation and/or endocytosis of the VEGF receptor, blocking yet another biologically active arm of VEGF/VEGF-R complex. Co-precipitation pull down assays could be used to deteremine what TSP-1 is interacting with directly. These studies could also yield important answers to when specific interactions are occurring in the angiogenic process, as the timeline of binding is unknown. Regardless of the mechanism, our data supports the notion 
that TSP-1 likely plays a regulatory role that also involves VEGF, thereby enhancing its already potent inherent anti-angiogenic capacity under physiological situations.

Unlike chapter 3 , the data in chapter 5 does not directly support the theory of a TSP1/VEGF balance, however it is not inconsistent. While it trended downward, we did not see any change in VEGF in either of the muscles tested in chapter 5, as well as no change in TSP-1 as discussed previously. While not what we anticipated, this null change in VEGF and TSP-1 still fits within the context of the balance theory. That is, no change in the negative protein TSP-1 would therefore spur no change in the positive angiogenic protein VEGF, with the converse also being true. The lack of change in VEGF also suggests, as we argue for TSP-1, that there may be divergent pathways that regulate physiological vs. pathological angiogenesis, despite previous data to suggest VEGFs role in pathological skeletal muscle dysfunction [49]. Still, since there was a significant decrease in skeletal muscle capillarity in chapter 5 (yet no change in TSP-1 or VEGF) our data suggests that some other mediator(s), potentially specific to chronic systemic inflammation induced capillary loss, may be at play with chronic systemic inflammation that may or may not be common to physiologically induced angiogenesis. Little information exists as to which negative angiogenic proteins are most important in skeletal muscle angiogenesis [50], so more work will need to be done to identity which proteins are important in TNF $\alpha$ driven chronic systemic inflammation.

To try and answer what proteins could be responsible for this pathological/inflammation induce capillary regression, we conducted a proteomic angiogenic array in chapter 5. Interestingly, we found that TSP-2 was the only protein of the 53 tested that was significantly changed (up by over $80 \%$ in the TNF $\alpha+$ animals). As discussed in chapter 1 (literature review) TSP-1 belongs to a family of six different proteins that all share some homology and functional 
overlap [51-53]. Of those, TSP-1 and TSP-2 are the most closely related in terms of both form and function, with TSP-2 only lacking a limited number of the distinct regions of TSP-1, such as the TGF- $\beta$ activating region [8]. TSP-2 has been shown to be anti-angiogenic in skeletal muscle [54], however its role in skeletal muscle is poorly studied. Further work will be needed to clarify if TSP-2 is a central player in inflammatory induced capillary regression. Still, we suggest here for the first time that TSP-2 could be the link between chronic systemic inflammation and skeletal muscle capillary rarefaction, thereby serving as a therapeutic target for the treatment of this important comorbidity in chronic disease.

Given that TSP-2 is up-regulated in chapter 5, and VEGF is trending downwards or unchanged, this still agrees with the hypothesis that an altered angiogenic balance controls vascular growth, as seen in skeletal muscle under physiological settings (as in chapter 3 and other studies $[6,38])$. It is important to point out that while there is one publication showing increased skeletal muscle capillarity with the knock-out of TSP-2 [55] no further work has been done to build upon this. Therefore, it has yet to be answered if TSP-2 has a controlling role in physiological or exercise induced capillary growth/regression (vs. pathological angiogenesis). It would be interesting to see if TSP-2 has a decreased role in physiological angiogenesis, given TSP-1's apparent dominant role. Studies using the dual TSP-1/TSP-2 knockout suggests that wound healing is dictated by TSP-1 and not TSP-2 [56], which might suggest further that under normal (physiological) conditions TSP-1 is more important compared to TSP-2. However, interestingly TSP-2 seems to be acting through the same CD36 receptor as TSP-1 in cancer, so the differences may be subtle [57]. If TSP-2 is indeed less important in physiological angiogenesis, but more important in pathological angiogenesis, it could suggest distinct and pivotal roles for the two closely related TSP family members. 
We also observed that in the angiogenic array all of the 53 proteins tested, both positive and negative, are trending upwards in the TNF $\alpha+$ animal. Indeed, this includes 13 proteins with $\mathrm{P}$ values of less than 0.10 . This may suggest that under a chronic systemic inflammatory challenge with TNF $\alpha$, as seen in our model, skeletal muscle could be in a constant state of angiogenic stimulus. The increase in TNF $\alpha$ could be driving an increase in TSP-2 expression, and that of other potentially yet-unknown negative angiogenic markers (like IL-10, IP-10, or MMP-9 as suggested by the angiogenic array), resulting in a decrease in capillarity, and a rebound response in positive angiogenic proteins. We hypothesize that constant elevated TNF $\alpha$ is increasing negative angiogenic factors causing a feed-forward loop. As the muscle reacts to the drop in capillarity caused by this increase in negative angiogenic proteins, it could spur a high expression of positive angiogenic factors as a compensatory mechanism. This could then further stimulate an increase in negative proteins, causing a feed-forward consequence. This could explain the general trend for increases in all angiogenic proteins. In this way, the balance is constantly trying to be reestablished, potentially resulting in a state of high-angiogenic protein production. Supporting this theory, preliminary work from our lab shows that both TSP-1 and VEGF are increased with physical detraining, even after capillarity is returned to baseline (figure 3 of appendix). It could be that an increase in TSP-1 drives the decrease in capillarity seen in that study, and VEGF is responding (increasing) to prevent too much capillary rarefaction, or slow the rarefaction, from occurring. In this way, VEGF would be serving as a feedback mechanism. Without a constant input, like that from TNF $\alpha$ as in chapter 5, this allows the system to be tightly regulated. It is only when you have this constant TNF $\alpha$ mediated chronic systemic inflammatory insult that you push the system into a feed-forward response. This is an intriguing hypothesis that will need further evidence for support. 
Connecting this notion to those findings in chapter 3, endogenous TSP-1 and its CD36 receptor are trending downward in response to exogenous chronic stimulation of a negative angiogenic pathway (TSP-1). As discussed previously, VEGF is also decreased in these studies of chapter 3. This suggests that skeletal muscle is reacting to an increase in the stimulation of the TSP-1 anti-angiogenic pathway, and the decreased expression of VEGF, by altering expression of endogenous TSP-1 and its receptors, likely in an effort to maintain homeostasis. This would be a similar feedback system as argued previously. While we admit in chapter 3 it could simply be that exogenous TSP-1 is directly binding and resulting in the endocytosis of VEGF (as argued previously in this discussion), these data is not inconsistent with this theory of a tightly controlled balance.

Regardless, chapters 3 and 5, in combination with the available literature, not only point to TSP-1 and TSP-2 as important components in controlling skeletal muscle angiogenesis, but also build upon and add significantly to the hypothesis that skeletal muscle capillarity is regulated by a balance between negative and positive angiogenic proteins.

\section{TSP-1 appears to affect oxidative muscles preferentially}

It is interesting to point out that in chapter 3 soleus capillarity is affected to a greater degree than that of the plantaris or gastrocnemius. While it is true that capillarity of the gastrocnemius and plantaris are both decreased in chapter 3, the soleus has the largest reduction in capillary-to-fiber ratio (35\% reduction in the soleus vs. $20 \%$ in the gastrocnemius and $15 \%$ in the plantaris). Interestingly, the gastrocnemius has the second greatest decrease, and it is the second most oxidative of the three muscles, with the plantaris being the least. This may suggest that TSP-1 affects slow-fiber type muscles (i.e. oxidative) to a larger extent than the fast-fiber 
muscles (i.e. glycolytic), as the soleus is comprised of a significantly greater proportion of slow muscle fibers when compared to either of the other muscles tested (i.e. type I and type IIa). Indeed, supporting this theory, in previous studies soleus capillarity in hind-limb unloading seems to be affected to a greater extent when compared to the plantaris [6], and only the soleus shows an increase in TSP-1 in this same model (again compared to the plantaris).

Paralleling the findings in chapter 3 , chapter 5 provides further proof that the soleus is the muscle preferentially affected. In chapter 5 the soleus is the only muscle with capillary reduction. However, potentially contrary to our hypothesis that TSP-1 may be driving this muscle-specific response, we only found a change in TSP-2, and not TSP-1, of the soleus in chapter 5. This may suggest the preferential loss of capillaries is purely based on the fact that this muscle (soleus) generally has more capillaries, so may have a greater capacity to lose vasculature without affecting function. That is, the soleus may be capable of absorbing some of the vascular loss because of its already high inherent capillarity. However, this seems unlikely based on previous findings showing that there is decreased muscle function in the soleus [21, 22].

It is more likely that there is a greater sensitivity in the soleus to the various forms of angiogenic stimuli (e.g. hypoxia, sheer stress, pH, etc.). This could be related to the soleus having higher endothelial cell content, as endothelial cells are known to be angiogenic sensors, and significant contributors of angiogenic proteins [58-60]. Interestingly, when compared between the different muscles under untreated conditions in chapter 3, VEGF is significantly higher in the soleus when compared to the plantaris $(\mathrm{P}<0.01)$, and trends higher in the gastrocnemius $(\mathrm{P}=0.17)$. This is also true of TSP-1 in chapter 5, which trends to be higher under basal conditions in the soleus when compared to the plantaris $(\mathrm{P}=0.2)$. This may mean the soleus 
has the greatest capacity for changes in angiogenic protein expression, which could explain its relative sensitivity to capillary growth/loss, as suggested previously [6].

These differential effects on the soleus may explain why we do not see exercise/functional differences in chapters 3 and 5 as well. In chapter 3 we do not see a change in maximal running speed. While this has been shown to be an appropriate exercise model in other studies previously $[3,61,62]$, it is limited in two important ways. First, it is a measurement of the whole-body exercise capacity of the animal, and secondly it can be potentially limited by the biomechanics of the animal. If it is true that oxidative muscles are more affected than glycolytic then it may also be true that the aerobic/endurance systems are also more affected, and hence these systems should be identified and tested. It may be that in our studies it is more appropriate to use endurance testing, where the animals are put on a treadmill and allowed to run for as long as possible at a set speed. Indeed, we decided to use endurance testing in chapter 5 , based on this theory and the findings in chapter 3 , and found a significant decrease in endurance time of $\mathrm{TNF} \alpha+$ mice, despite previous evidence suggesting no change in maximal running speed [22]. Supporting these findings further, in vivo muscle stimulation which tests the whole tricep surea yielded no significant difference in muscle fatigue between the $\mathrm{TNF} \alpha+$ and control mice. Since the plantaris was shown to have no change in capillarity, and preliminary evidence suggests the gastrocnemius is also unchanged (not shown), and the soleus makes up the smallest contribution to the hindlimb muscle triad, it may not be surprising we saw no difference in function. This would then agree with previous findings, where Tang et al. showed in $\mathrm{TNF} \alpha+$ mice soleus fatigue was increased, while the EDL remained unchanged in situ [22], again suggesting an oxidative specific response. 
In summary, it appears that there is a capillary-specific loss in the soleus. This could be significant in terms of overall function, and muscle function, in humans. We rely heavily upon our aerobic muscles for daily tasks such as climbing stairs and walking. In concert with a reduced exercise capacity from central pathological contributors found in many chronic diseases (e.g. lung function, cardiac output) [25-27, 63-65], even a small additional loss in capillarity/oxidative capacity of skeletal muscle could translate into large functional deficits. In COPD there is a documented reduction in muscle function, $\mathrm{VO}_{2 \max }$, capillarity, and the number of type I fibers in human quadriceps muscle [24, 27]. The loss of type I fibers is correlated with a decrease in overall exercise capacity more strongly in those with COPD than healthy controls [27], thus pointing towards an important role for this potential muscle specific reduction in capillarity. Despite the work here, what role TSP-1 plays in this specific response remains unclear, however chapter 3 points towards a potential regulatory role.

\section{The role of TSP-1 in skeletal muscle endothelial cell apoptosis remains unclear}

TSP-1 has been shown to be promote apoptosis acting through multiple mechanisms, including the CD36 receptor [66-71]. Endothelial apoptosis is a naturally occurring mechanism by which capillary regression can take place [72-75], and myocyte apoptosis has been shown to be central in muscle atrophy under some pathological conditions as well as aging and disuse [7680]. However, the role of TSP-1 and CD36 in myocyte and endothelial cell apoptosis in vivo is not well characterized.

In chapter 3 we performed TUNEL staining to look at end stage apoptosis (DNA fragmentation) in response to a chronic administration of the TSP-1/CD36 agonist ABT-510. Surprisingly, we found no evidence of apoptosis in any of the muscles, either in myocyte or 
endothelial cells. The reason for this remains unclear. This is, however, the first in vivo report of ABT-510s affects on skeletal muscle and it could be that ABT-510 is not sufficient to induce myocyte apoptosis in skeletal muscle. This is supported by a lack of change in muscle fiber cross-sectional area (FCSA) in both chapters $3 \& 4$, despite both long and short-term chronic doses of the TSP-1 mimetic in each study, respectively. However, much of the work with TSP-1 and apoptosis pertains to cancer models (e.g. tumorigenesis), endothelial cells, and is typically done in vitro $[66,67,70,81]$. Despite circumstantial evidence suggesting that removal of TSP1/CD47 can protect skeletal muscle from radiation injury [82], it could be that a TSP-1/CD36 mechanism does not play a role in skeletal muscle apoptosis.

However, this does not explain why we saw no endothelial cell apoptosis in chapters 3 or 4. However, chapter 3 examined only one time point. We hypothesized that apoptosis could have occurred at an earlier time point than our single assessment at 14 days. Indeed, it has been shown previously that hindlimb unloading results in capillary loss in as little as 5 days [6]. For this reason in chapter 4 we hypothesized that evidence of endothelial cell death would be present in the muscles of ABT-510 treated mice at 3 and/or 7 days. Surprisingly, we still found no evidence of apoptosis, or necrosis (cell death), with either of these shorter time points. The data from chapter 3 and chapter 4 together imply that the reduction in muscle capillarity that occurs with chronic exposure to a TSP-1 mimetic is not mediated by a traditional cell death mechanism (e.g. apoptosis). This is an intriguing and novel finding that could have impact on the direction of further research into this area.

It could be that there are increased levels of autophagy in response to the TSP-1 mimetic, resulting ultimately in cell dysfunction and death. Autophagy has been shown to be important in other systems, including skeletal muscle [83-85]. While not directly linked to CD36, recently 
the TSP-1 receptor CD47 has been implicated in autophagy, where CD47 agonists increase autophagy in cancer cells, as well as decreasing the capillary supply of tumors, all without causing any apoptosis [86]. It has also been shown that other anti-angiogenic proteins, such as endostatin, can trigger autophagy which results in inhibition of angiogenesis [87]. Interestingly, endostatin has been shown to up-regulate TSP-1, but what role this connection has in autophagy remains to be seen $[88,89]$. While only speculative at this point, autophagy could be a nonapoptotic mechanism for the removal of endothelial cells in skeletal muscle, or reduction in their viability, and hence a resultant shrinking of the capillary network.

It is also important to point out possible experimental limitations in our methods to measure apoptosis in chapters $3 \& 4$. While TUNEL has been a time-tested quality measurement of end stage apoptosis in many models [90-93], it only captures those nuclei undergoing DNA fragmentation. With the number of endothelial cell nuclei being relatively low in a $10 \mu \mathrm{m}$ serial muscle section, it could be that we are simply not capturing enough sample to see significant apoptosis occurring. But it does seem unlikely that the number of apoptotic nuclei would be significantly lower between groups and yet undetectable, given previous findings using similar methods [94].

This is why we chose the cell death ELISA in chapter 4, as it does not rely on 1 single snap shot of a muscle section, but rather captures what is going on in the entirety of the muscle. We hypothesized then that it would identify all cells undergoing cell death (including all endothelial cells) found in the tissue (skeletal muscle). However, the ELISA is limited in that it does not specifically measure apoptosis, but measures total cell death (apoptosis, necrosis). Still, it has been used to quantify endothelial cell death previously [95]. Regardless of these 
limitations, we argue that the combination of the TUNEL staining and the ELISA together establish the lack of apoptosis in skeletal muscle endothelial cells in chapters 3 and 4.

\section{TSP-1 influences mitochondrial enzyme activity}

It was shown previously that TSP-1 can control mitochondrial biogenesis in young animals [96] and that TSP-1 KO animals have more intact mitochondria after an 8 weeks of treatment with radiation compared with wild-type animals [82]. While this suggests a regulatory role for TSP-1 in mitochondrial biogenesis and integrity, both of these studies are missing any direct measurement of mitochondrial function. In chapter 4 we showed that mitochondrial electron transport chain (mETC) enzyme activity is decreased in response to increased TSP-1, and increased in TSP-1 KO. In combination with decreasing capillarity, TSP-1's control of mitochondrial function could be an additive response in its control of total muscle function. That is, mitochondria have been shown to be vital in total exercise capacity [65, 97-102], and regulation of their oxidative capacity would therefore have a significant role in overall muscle function. While mitochondria have been shown to be regulated by many different proteins, TSP1 could be an important player in this process [96, 103-105].

The mechanism by which TSP-1 decreases mETC activity in chapter 4 is unknown. It has been suggested that it could be due to TSP-1's control of nitric oxide (NO) [96, 106-109]. NO is a stimulator of mitochondrial biogenesis [110-112]. However, TSP-1's control of NO has been shown to occur through its CD47 receptor [107, 113, 114]. In our studies in chapter 3 and 4, we did not investigate CD47, rather we targeted the TSP-1/CD36 axis to examine how it affected capillarity and mitochondria function. The work shown here is the first evidence that TSP-1 may be influencing mitochondria function through TSP-1/CD36. There is only one other 
study looking at CD36s role in mitochondria oxidative function, and that study found no difference in mitochondrial fatty acid enzyme activity in a whole-body CD36 KO [115]. Interestingly, CD36 has been found in the mitochondrial membrane, where it is thought to control fatty acid translocation [116]. Whether TSP-1 is binding cell surface receptors and triggering a protein cascade, or is being translocated and binding CD36 on the mitochondria directly, is yet unknown.

It could be that the decrease in mETC activity is simply due to a decrease in mitochondria number (i.e. less mitochondria, less total enzyme), as shown previously [96]. Long term hypoxia has also been shown previously to decrease mitochondrial density $[117,118]$, and so the antiangiogenic actions of TSP-1 could also be indirectly influencing mitochondria density and function. The lack of any difference in the CD36 KO mitochondrial function $[115,116]$, but a decrement in our TSP-1 exposure model in chapter 3, may suggest that this is truly a TSP-1 mediated function, and not a CD36 function per se. It also supports the previous notion that perhaps TSP-1's regulation of mitochondria is controlled by CD47's regulation of nitric oxide [96], and not by CD36. Further studies directed at TSP-1, CD36, CD47, will provide answers to these questions.

Given the change in mitochondrial enzyme activity, we hypothesized that muscle function and whole body exercise capacity would be decreased with changes in TSP-1. However, in chapters 3 we show no change in overall exercise capacity in terms of maximal running speed, and in chapter 4 we show no change in ex vivo muscle function. It could be that the changes we see are not enough to result in isolated muscle/whole body functional differences; however this seems unlikely, because the changes in mitochondrial enzyme activity found here are larger than those shown previously to occur with six weeks of exercise training 
[119]. Further, a decrease in soleus capillarity of the magnitude found in chapter 3 is at least equal in magnitude to the increase in capillarity that exercise training stimulates. This magnitude of change has been shown previously by our lab and others to affect overall exercise function [120-122], and is greater in magnitude than that seen with 9 days of hindlimb unloading [6]. Therefore we must conclude that this is a functionally relevant decrease. It is much more likely that while maximal running speed has been shown to be a good surrogate for $\mathrm{VO}_{2 \max }$ in mice, as discussed previously, it may not be the best test to have performed in the context of this study (i.e. an endurance run test could have been a more appropriate test).

This same concept could also be applied to the ex vivo preparation. The strength of ex vivo muscle stimulation is that it removes central factors from the regulation of skeletal muscle function (i.e. the heart and lungs), and focuses solely on the muscle. However, it is not the best reflection of the aerobic/endurance systems of the muscle. That is, because the endogenous vascular network is removed in the process of excising the muscle, ex vivo stimulation divorces blood flow from its influence on muscle function. Still, it is surprising we saw no change in ex vivo function in the TSP-1 KOs, as they have been shown to have increased endurance capacity as well as increased maximal running speed [3]. Of course, the mETC activities that we tested only make up one part of the oxidative capacity of the mitochondria, and it could be that the change in these specific enzymes are not enough to cause an overall change, especially since we are removing the muscles from their endogenous system. The changes in the mETC enzymes may only be functionally relevant with the corresponding changes in vascularity (i.e. increased $\mathrm{C}: \mathrm{F}$ in the $\mathrm{KO}$ and decreased $\mathrm{C}: \mathrm{F}$ in the mimetic treated animals) since the ex vivo preparation provides a super-physiologic amount of metabolic substrate, and divorces endogenous blood flow. In situ or in vivo muscle stimulation could answer these questions, as they keep the 
endogenous vascular and metabolic systems intact. Indeed, the reason we chose to do in vivo muscle stimulation in chapter 6 is precisely because of the findings in chapter 5 . If we want to answer what is occurring with aerobic exercise and muscle function in the future, these limitations will need to be addressed. 


\section{Citations}

1. Good, D.J., et al., A tumor suppressor-dependent inhibitor of angiogenesis is immunologically and functionally indistinguishable from a fragment of thrombospondin. Proceedings of the National Academy of Sciences, 1990. 87(17): p. 6624-6628.

2. Olfert, I.M., et al., Temporal thrombospondin-1 mRNA response in skeletal muscle exposed to acute and chronic exercise. Growth Factors, 2006. 24(4): p. 253-259.

3. Malek, M.H. and I.M. Olfert, Global deletion of thrombospondin 1 increases cardiac and skeletal muscle capillarity and exercise capacity in mice. Experimental physiology, 2009. 94(6): p. 749-760.

4. Malek, M.H., et al., Similar skeletal muscle angiogenic and mitochondrial signalling following 8 weeks of endurance exercise in mice: discontinuous versus continuous training. Experimental physiology, 2013.98(3): p. 807-818.

5. Gouzi, F., et al., Blunted muscle angiogenic training-response in copd patients versus sedentary controls. European Respiratory Journal, 2012.

6. Roudier, E., et al., Angio-adaptation in unloaded skeletal muscle: new insights into an early and muscle type-specific dynamic process. The Journal of Physiology, 2010. 588(22): p. 4579.

7. Kivela, R., et al., Exercise-induced expression of angiogenic growth factors in skeletal muscle and in capillaries of healthy and diabetic mice. Cardiovascular Diabetology, 2008. 7(1): p. 13.

8. Lawler, J., The functions of thrombospondin-1 and-2. Current opinion in cell biology, 2000. 12(5): p. 634-640.

9. Zhang, X. and J. Lawler, Thrombospondin-based antiangiogenic therapy. Microvascular research, 2007. 74(2-3): p. 90-99.

10. Lawler, J. and M. Detmar, Tumor progression: the effects of thrombospondin-1 and -2. The International Journal of Biochemistry \&amp; Cell Biology, 2004. 36(6): p. 10381045.

11. Ren, B., et al., Regulation of tumor angiogenesis by thrombospondin-1. Biochimica et Biophysica Acta (BBA) - Reviews on Cancer, 2006. 1765(2): p. 178-188.

12. Febbraio, M., D.P. Hajjar, and R.L. Silverstein, CD36: a class B scavenger receptor involved in angiogenesis, atherosclerosis, inflammation, and lipid metabolism. Journal of Clinical Investigation, 2001. 108(6): p. 785-792.

13. Danesh, J., et al., Low grade inflammation and coronary heart disease: prospective study and updated meta-analyses. BMJ, 2000. 321(7255): p. 199-204.

14. Gosker, H.R., et al., Skeletal muscle dysfunction in chronic obstructive pulmonary disease and chronic heart failure: underlying mechanisms and therapy perspectives. The American Journal of Clinical Nutrition, 2000. 71(5): p. 1033-1047.

15. $\mathrm{Xu}, \mathrm{H}$., et al., Chronic inflammation in fat plays a crucial role in the development of obesity-related insulin resistance. The Journal of Clinical Investigation, 2003. 112(12): p. 1821-1830.

16. Duncan, B.B., et al., Low-Grade Systemic Inflammation and the Development of Type 2 Diabetes. Diabetes, 2003. 52(7): p. 1799-1805.

17. Wouters, E.F.M., E.C. Creutzberg, and A.M.W.J. Schols, SYstemic effects in copd*. CHEST Journal, 2002. 121(5_suppl): p. 127S-130S. 
18. Kapadia, S.R., et al., Elevated circulating levels of serum tumor necrosis factor-alpha in patients with hemodynamically significant pressure and volume overload. Journal of the American College of Cardiology, 2000. 36(1): p. 208-212.

19. Feldman, A.M., et al., The role of tumor necrosis factor in the pathophysiology of heart failure. Journal of the American College of Cardiology, 2000. 35(3): p. 537-544.

20. Miyazaki, Y., et al., Expression of a tumor necrosis factor-alpha transgene in murine lung causes lymphocytic and fibrosing alveolitis. A mouse model of progressive pulmonary fibrosis. J Clin Invest, 1995. 96(1): p. 250-9.

21. Zuo, L., L. Nogueira, and M.C. Hogan, Effect of pulmonary TNF-alpha overexpression on mouse isolated skeletal muscle function. Am J Physiol Regul Integr Comp Physiol, 2011. 301(4): p. R1025-31.

22. Tang, K., et al., Impaired exercise capacity and skeletal muscle function in a mouse model of pulmonary inflammation. J Appl Physiol, 2013. 114(9).

23. Serres, I., et al., Impaired skeletal muscle endurance related to physical inactivity and altered lung function in COPD patients. Chest, 1998. 113(4): p. 900-5.

24. Jobin, J., et al., Chronic obstructive pulmonary disease: capillarity and fiber-type characteristics of skeletal muscle. J Cardiopulm Rehabil, 1998. 18(6): p. 432-7.

25. Clark, C., et al., Skeletal muscle strength and endurance in patients with mild COPD and the effects of weight training. European Respiratory Journal, 2000. 15(1): p. 92-97.

26. Casaburi, R., Skeletal Muscle Function in COPD*. Chest, 2000. 117(5 suppl 1): p. 267S$271 \mathrm{~S}$.

27. Allaire, J., et al., Peripheral muscle endurance and the oxidative profile of the quadriceps in patients with COPD. Thorax, 2004. 59(8): p. 673-678.

28. Man, W., et al., Skeletal muscle dysfunction in COPD: clinical and laboratory observations. Clinical Science, 2009. 117: p. 251-264.

29. Burtin, C., et al., Effectiveness of exercise training in patients with COPD: the role of muscle fatigue. Eur Respir J, 2012. 40(2): p. 338-44.

30. Eliason, G., et al., Research Alterations in the muscle-to-capillary interface in patients with different degrees of chronic obstructive pulmonary disease. 2010.

31. Di Francia, M., et al., Tumor necrosis factor-alpha levels and weight loss in chronic obstructive pulmonary disease. Am J Respir Crit Care Med, 1994. 150(5 Pt 1): p. 1453-5.

32. Groenewegen, K.H., et al., Increased systemic inflammation is a risk factor for COPD exacerbations. CHEST Journal, 2008. 133(2): p. 350-357.

33. Tang, K., P.D. Wagner, and E.C. Breen, TNF-alpha-mediated reduction in PGC-1alpha may impair skeletal muscle function after cigarette smoke exposure. J Cell Physiol, 2010. 222(2): p. 320-7.

34. Salajegheh, M., et al., Upregulation of thrombospondin-1 (TSP-1) and its binding partners, CD36 and CD47, in sporadic inclusion body myositis. Journal of neuroimmunology, 2007. 187(1-2): p. 166-174.

35. Hotamisligil, G.S. and B.M. Spiegelman, Tumor necrosis factor $\alpha$ : a key component of the obesity-diabetes link. Diabetes, 1994. 43(11): p. 1271-1278.

36. Kuschner, W., et al., Dose-dependent cigarette smoking-related inflammatory responses in healthy adults. European Respiratory Journal, 1996. 9(10): p. 1989-1994.

37. Frisbee, J.C., Remodeling of the skeletal muscle microcirculation increases resistance to perfusion in obese Zucker rats. American Journal of Physiology-Heart and Circulatory Physiology, 2003. 285(1): p. H104-H111. 
38. Audet, G.N., et al., Expression of angiogenic regulators and skeletal muscle capillarity in selectively bred high aerobic capacity mice. Experimental physiology, 2011. 96(11): p. 1138-1150.

39. Greenaway, J., et al., Thrombospondin-1 inhibits VEGF levels in the ovary directly by binding and internalization via the low density lipoprotein receptor-related protein-1 (LRP-1). Journal of cellular physiology, 2007. 210(3): p. 807-18.

40. Gupta, K., et al., Binding and displacement of vascular endothelial growth factor (VEGF) by thrombospondin: Effect on human microvascular endothelial cell proliferation and angiogenesis. Angiogenesis, 1999. 3(2): p. 147-158.

41. Iruela-Arispe, M.L., et al., Inhibition of Angiogenesis by Thrombospondin-1 Is Mediated by 2 Independent Regions Within the Type 1 Repeats. Circulation, 1999. 100(13): p. 1423-1431.

42. Rodríguez-Manzaneque, J.C., et al., Thrombospondin-1 suppresses spontaneous tumor growth and inhibits activation of matrix metalloproteinase-9 and mobilization of vascular endothelial growth factor. Proceedings of the National Academy of Sciences of the United States of America, 2001. 98(22): p. 12485.

43. Sun, J., et al., Thrombospondin-1 modulates VEGF-A-mediated Akt signaling and capillary survival in the developing retina. American Journal of Physiology-Heart and Circulatory Physiology, 2009. 296(5): p. H1344.

44. Simons, M., An Inside View: VEGF Receptor Trafficking and Signaling. Physiology, 2012. 27(4): p. 213-222.

45. Kaur, S., et al., Thrombospondin-1 Inhibits VEGF Receptor-2 Signaling by Disrupting Its Association with CD47. Journal of Biological Chemistry, 2010. 285(50): p. 38923-38932.

46. Dawson, D.W., et al., CD36 mediates the in vitro inhibitory effects of thrombospondin-1 on endothelial cells. The Journal of cell biology, 1997. 138(3): p. 707.

47. Zhang, X., et al., Thrombospondin-1 modulates vascular endothelial growth factor activity at the receptor level. The FASEB journal, 2009. 23(10): p. 3368-3376.

48. Primo, L., et al., Identification of CD36 molecular features required for its in vitro angiostatic activity. The FASEB journal, 2005. 19(12): p. 1713-1715.

49. Barreiro, E., et al., Cytokine profile in quadriceps muscles of patients with severe COPD. Thorax, 2008. 63(2): p. 100-107.

50. Mark Olfert, I. and O. Birot, Importance of anti angiogenic factors in the regulation of skeletal muscle angiogenesis. Microcirculation, 2011.

51. Mosher, D.F., Physiology of thrombospondin. Annual review of medicine, 1990. 41(1): p. 85-97.

52. Jack, L., The functions of thrombospondin-1 and-2. Current Opinion in Cell Biology, 2000. 12(5): p. 634-640.

53. Bornstein, P., Thrombospondins function as regulators of angiogenesis. Journal of Cell Communication and Signaling, 2009. 3(3): p. 189-200.

54. Reinecke, H., et al., Lack of thrombospondin-2 reduces fibrosis and increases vascularity around cardiac cell grafts. Cardiovasc Pathol, 2013. 22(1): p. 91-5.

55. Krady, M.M., et al., Thrombospondin-2 modulates extracellular matrix remodeling during physiological angiogenesis. The American Journal of Pathology, 2008. 173(3): p. 879-891. 
56. Agah, A., et al., The lack of thrombospondin-1 (TSP1) dictates the course of wound healing in double-TSP1/TSP2-null mice. The American Journal of Pathology, 2002. 161(3): p. 831-839.

57. Koch, M., et al., CD36-mediated activation of endothelial cell apoptosis by an $\mathrm{N}$ terminal recombinant fragment of thrombospondin-2 inhibits breast cancer growth and metastasis in vivo. Breast Cancer Res Treat, 2011. 128(2): p. 337-46.

58. Munoz-Chapuli, R., A. Quesada, and M.A. Medina, Angiogenesis and signal transduction in endothelial cells. Cellular and Molecular Life Sciences CMLS, 2004. 61(17): p. 2224-2243.

59. Folkman, J., Editorial: Is tissue mass regulated by vascular endothelial cells? Prostate as the first evidence. Endocrinology, 1998. 139(2): p. 441-442.

60. Pohl, U., Endothelial cells as part of a vascular oxygen-sensing system: hypoxia-induced release of autacoids. Experientia, 1990. 46(11-12): p. 1175-1179.

61. Olfert, I.M., et al., Muscle-specific VEGF deficiency greatly reduces exercise endurance in mice. The Journal of Physiology, 2009. 587(8): p. 1755.

62. Olfert, I.M., et al., Myocyte vascular endothelial growth factor is required for exerciseinduced skeletal muscle angiogenesis. American Journal of Physiology - Regulatory, Integrative and Comparative Physiology, 2010. 299(4): p. R1059-R1067.

63. Sullivan, M.J. and M.H. Hawthorne, Exercise intolerance in patients with chronic heart failure. Progress in Cardiovascular Diseases. 38(1): p. 1-22.

64. Harrington, D., et al., Skeletal Muscle Function and Its Relation to Exercise Tolerance in Chronic Heart Failure. Journal of the American College of Cardiology, 1997. 30(7): p. 1758-1764.

65. Phielix, E., et al., Exercise training increases mitochondrial content and ex vivo mitochondrial function similarly in patients with type 2 diabetes and in control individuals. Diabetologia, 2010. 53(8): p. 1714-1721.

66. Friedl, P., P. Vischer, and M. Freyberg, The role of thrombospondin-1 in apoptosis. Cellular and molecular life sciences, 2002. 59(8): p. 1347-1357.

67. Guo, N., et al., Thrombospondin 1 and type I repeat peptides of thrombospondin 1 specifically induce apoptosis of endothelial cells. Cancer research, 1997. 57(9): p. 1735.

68. Jiménez, B., et al., Signals leading to apoptosis-dependent inhibition of neovascularization by thrombospondin-1. Nature medicine, 2000. 6(1): p. 41-48.

69. Miao, W.-M., et al., Thrombospondin-1 Type 1 Repeat Recombinant Proteins Inhibit Tumor Growth through Transforming Growth Factor- $\beta$-dependent and-independent Mechanisms. Cancer research, 2001. 61(21): p. 7830-7839.

70. Nör, J., et al., Thrombospondin-1 induces endothelial cell apoptosis and inhibits angiogenesis by activating the caspase death pathway. Journal of vascular research, 2000. 37(3): p. 209-218.

71. Rege, T.A., et al., Thrombospondin-1-induced apoptosis of brain microvascular endothelial cells can be mediated by TNF-Rl. Journal of cellular physiology, 2009. 218(1): p. 94-103.

72. Meeson, A.P., et al., VEGF deprivation-induced apoptosis is a component of programmed capillary regression. Development, 1999. 126(7): p. 1407-1415.

73. Tang, K., et al., Capillary regression in vascular endothelial growth factor-deficient skeletal muscle. Physiological Genomics, 2004. 18(1): p. 63-69. 
74. Meeson, A., et al., A relationship between apoptosis and flow during programmed capillary regression is revealed by vital analysis. Development, 1996. 122(12): p. 39293938.

75. Dimmeler, S. and A.M. Zeiher, Endothelial Cell Apoptosis in Angiogenesis and Vessel Regression. Circulation research, 2000. 87(6): p. 434-439.

76. Degens, H. and S.E. Alway, Control of muscle size during disuse, disease, and aging. International journal of sports medicine, 2006. 27(2): p. 94-99.

77. Siu, P.M., et al., Aging influences cellular and molecular responses of apoptosis to skeletal muscle unloading. American Journal of Physiology - Cell Physiology, 2005. 288(2): p. C338-C349.

78. dalla Libera, L., et al., Apoptosis and atrophy in rat slow skeletal muscles in chronic heart failure. American Journal of Physiology - Cell Physiology, 1999. 277(5): p. C982C986.

79. Dupont-Versteegden, E.E., Apoptosis in muscle atrophy: Relevance to sarcopenia. Experimental Gerontology, 2005. 40(6): p. 473-481.

80. Allen, D.L., et al., Apoptosis: a mechanism contributing to remodeling of skeletal muscle in response to hindlimb unweighting. American Journal of Physiology - Cell Physiology, 1997. 273(2): p. C579-C587.

81. Guo, N.-h., et al., Thrombospondin 1 and Type I Repeat Peptides of Thrombospondin 1 Specifically Induce Apoptosis of Endothelial Cells. Cancer research, 1997. 57(9): p. 1735-1742.

82. Isenberg, J.S., et al., Thrombospondin-1 and CD47 limit cell and tissue survival of radiation injury. The American Journal of Pathology, 2008. 173(4): p. 1100-1112.

83. Kim, Y.A., et al., Autophagic response to exercise training in skeletal muscle with age. Journal of Physiology and Biochemistry, 2013: p. 1-9.

84. Marino, G. and C. Lopez-Otin, Autophagy: molecular mechanisms, physiological functions and relevance in human pathology. Cellular and Molecular Life Sciences CMLS, 2004. 61(12): p. 1439-1454.

85. Klionsky, D.J. and S.D. Emr, Autophagy as a regulated pathway of cellular degradation. Science, 2000. 290(5497): p. 1717-1721.

86. Kalas, W., et al., Thrombospondin-1 receptor mediates autophagy of RAS-expressing cancer cells and triggers tumour growth inhibition. Anticancer Res, 2013. 33(4): p. 142938.

87. Ramakrishnan, S., et al., Autophagy and angiogenesis inhibition. Autophagy, 2007. 3(5): p. 511-514.

88. Ding, I., et al., Intratumoral administration of endostatin plasmid inhibits vascular growth and perfusion in MCa-4 murine mammary carcinomas. Cancer research, 2001. 61(2): p. 526-531.

89. Folkman, J., Endogenous angiogenesis inhibitors. Apmis, 2004. 112(7-8): p. 496-507.

90. Pistilli, E.E., P.M. Siu, and S.E. Alway, Molecular regulation of apoptosis in fast plantaris muscles of aged rats. The Journals of Gerontology Series A: Biological Sciences and Medical Sciences, 2006. 61(3): p. 245-255.

91. Loo, D.T., In situ detection of apoptosis by the TUNEL assay: an overview of techniques, in DNA Damage Detection In Situ, Ex Vivo, and In Vivo. 2011, Springer. p. 3-13. 
92. White, L.E., et al., TNFR1-dependent pulmonary apoptosis during ischemic acute kidney injury. American Journal of Physiology-Lung Cellular and Molecular Physiology, 2012. 303(5): p. L449-L459.

93. Kim, S.-E., et al., Treadmill exercise prevents aging-induced failure of memory through an increase in neurogenesis and suppression of apoptosis in rat hippocampus.

Experimental Gerontology, 2010. 45(5): p. 357-365.

94. de Resende, M.M., et al., Role of endothelial cell apoptosis in regulation of skeletal muscle angiogenesis during high and low salt intake. Physiological Genomics, 2006. 25(2): p. 325-335.

95. Jung, C.H., et al., Vaspin protects vascular endothelial cells against free fatty acidinduced apoptosis through a phosphatidylinositol 3-kinase/Akt pathway. Biochemical and biophysical research communications, 2011. 413(2): p. 264-269.

96. Frazier, E.P., et al., Age-dependent regulation of skeletal muscle mitochondria by the thrombospondin-1 receptor CD47. Matrix Biology, 2011.

97. Hoppeler, H. and E.R. Weibel, Limits for oxygen and substrate transport in mammals. Journal of Experimental Biology, 1998. 201(8): p. 1051-64.

98. Hoppeler, H. and M. Fluck, Plasticity of skeletal muscle mitochondria: structure and function. Medicine and science in sports and exercise, 2003. 35(1): p. 95-104.

99. Yan, Z., et al., Regulation of exercise-induced fiber type transformation, mitochondrial biogenesis, and angiogenesis in skeletal muscle. Journal of Applied Physiology, 2011. 110(1): p. 264-274.

100. Gollnick, P.D. and D.W. King, Effect of exercise and training on mitochondria of rat skeletal muscle. American Journal of Physiology--Legacy Content, 1969. 216(6): p. 1502-1509.

101. Morgan, T., et al., Effects of long-term exercise on human muscle mitochondria, in Muscle metabolism during exercise. 1971, Springer. p. 87-95.

102. Bo, H., Y. Zhang, and L.L. Ji, Redefining the role of mitochondria in exercise: a dynamic remodeling. Annals of the New York Academy of Sciences, 2010. 1201(1): p. 121-128.

103. Hock, M.B. and A. Kralli, Transcriptional control of mitochondrial biogenesis and function. Annual review of physiology, 2009. 71: p. 177-203.

104. Nisoli, E., et al., Mitochondrial biogenesis in mammals: the role of endogenous nitric oxide. Science Signaling, 2003. 299(5608): p. 896.

105. Seppet, E., et al., Adaptation of Cardiac and Skeletal Muscle Mitochondria to Endurance Training: Implications for Cardiac Protection, in Cardiac Adaptations. 2013, Springer. p. $375-402$.

106. Isenberg, J., W. Frazier, and D. Roberts, Thrombospondins: from structure to therapeutics: Thrombospondin-1: a physiological regulator of nitric oxide signaling. Cellular and Molecular Life Sciences CMLS, 2008. 65(5): p. 728-742.

107. Isenberg, J.S., et al., CD47 is necessary for inhibition of nitric oxide-stimulated vascular cell responses by thrombospondin-1. Journal of Biological Chemistry, 2006. 281(36): p. 26069-26080.

108. Isenberg, J.S., et al., Thrombospondin-1 inhibits endothelial cell responses to nitric oxide in a cGMP-dependent manner. Proceedings of the National Academy of Sciences of the United States of America, 2005. 102(37): p. 13141. 
109. Ridnour, L.A., et al., Nitric oxide regulates angiogenesis through a functional switch involving thrombospondin-1. Proceedings of the National Academy of Sciences of the United States of America, 2005. 102(37): p. 13147-13152.

110. Stamler, J.S. and G. Meissner, Physiology of nitric oxide in skeletal muscle. Physiological Reviews, 2001. 81(1): p. 209-237.

111. Nisoli, E. and M.O. Carruba, Nitric oxide and mitochondrial biogenesis. Journal of cell science, 2006. 119(14): p. 2855-2862.

112. Lira, V.A., et al., Nitric oxide and AMPK cooperatively regulate PGC-1 $\alpha$ in skeletal muscle cells. The Journal of Physiology, 2010. 588(18): p. 3551-3566.

113. Brown, E.J. and W.A. Frazier, Integrin-associated protein (CD47) and its ligands. Trends in cell biology, 2001. 11(3): p. 130-135.

114. Isenberg, J.S., D.D. Roberts, and W.A. Frazier, CD47: a new target in cardiovascular therapy. Arteriosclerosis, thrombosis, and vascular biology, 2008. 28(4): p. 615.

115. Holloway, G.P., et al., FAT/CD36-null mice reveal that mitochondrial FAT/CD36 is required to upregulate mitochondrial fatty acid oxidation in contracting muscle. American Journal of Physiology-Regulatory, Integrative and Comparative Physiology, 2009. 297(4): p. R960-R967.

116. Bezaire, V., et al., Identification of fatty acid translocase on human skeletal muscle mitochondrial membranes: essential role in fatty acid oxidation. American Journal of Physiology-Endocrinology And Metabolism, 2006. 290(3): p. E509-E515.

117. Hoppeler, H. and M. Vogt, Muscle tissue adaptations to hypoxia. Journal of Experimental Biology, 2001. 204(18): p. 3133-3139.

118. Hoppeler, H., et al., Response of skeletal muscle mitochondria to hypoxia. Experimental physiology, 2003. 88(1): p. 109-119.

119. Rodriguez-Bies, E., et al., Muscle physiology changes induced by every other day feeding and endurance exercise in mice: effects on physical performance. PLoS One, 2010. 5(11): p. e13900.

120. Olenich, S.A.G.-R., Navarre, Audet, Gerald N., Olfert, Mark I., Temporal response of positive and negative regulators in response to acute and chronic exercise training in mice. The Journal of Physiology, 2013. Under Review.

121. Waters, R.E., et al., Voluntary running induces fiber type-specific angiogenesis in mouse skeletal muscle. American Journal of Physiology - Cell Physiology, 2004. 287(5): p. C1342-C1348.

122. Audet, G.N., et al., Chronic delivery of a thrombospondin-1 mimetic decreases skeletal muscle capillarity in mice. PLoS One, 2013. 8(2): p. e55953. 
This page intentionally left blank 


\section{Chapter 7: Future Directions}


Given the findings in all chapters of this dissertation, there are significant new questions that now will require further investigation.

One interesting future direction would be to analyze what role TSP-2 has in both pathological and physiological angiogenesis. It appears TSP-2 may be playing a role in pathological capillary regression, but the work in this dissertation is the first and only data to suggest this (chapter 5, figure 5). Indeed, there is almost no work on TSP-2 in skeletal muscle under any conditions, and the data that does exist does not directly analyze what role TSP-2 plays in skeletal muscle angiogenesis. The potential importance of TSP-2 becomes even more intriguing when taken in context with the finding that TSP-1 likely has little or no role in chronic systemic inflammatory induced capillary rarefaction, yet seems to play an important role in physiological angiogenesis (chapter 5). It would be interesting to look at TSP-2 in normal healthy animals, as well and also to determine what effects exercise training has on TSP-2. This could yield further information as to TSP-2s role in controlling physiological angiogenesis, as exercise is one of the strongest stimulus for angiogenesis. Further work with the TSP-2 KOs to see how their exercise capacity and skeletal muscle capillarity compares to normal animals would also yield powerful information not only on the role of TSP-2, but how TSP-2 compares to TSP-1 in this context. These potentially divergent roles for the closely related TSP-1 and TSP-2 proteins have not yet been characterized. Further, since capillary loss and an altered angiogenic response are strongly correlated with the muscle dysfunction in chronic disease finding a major regulator that could serve as either a) a biomarker or b) a therapeutic target of this dysfunction is of high interest to both scientists and clinicians alike. We suggest that TSP-2 could be this protein. 
We have eliminated TSP-1 and VEGF as potential markers/mediators of capillary regression in a TNF $\alpha$ induced chronic systemic inflammatory insult, despite their apparent critical roles in physiological angiogenesis. To date, we have speculated that the pathways that control pathological and physiological angiogenesis are regulated by the same protein mediators based on the available literature, however little evidence exists to either support or refute our hypothesis in skeletal muscle. Our data suggests that there may be differences in the regulatory pathways of skeletal muscle angiogenesis that relate to specific conditions. That is, we suggest that there are divergent pathways that control physiological vs. pathological angiogenesis in skeletal muscle. Identifying these different pathways and the molecular mediators important to each could be important in a variety of settings, from exercise training prescription to clinical treatment of disease. This suggests future work with broad-scope proteomics may help to identify how divergent pathways and proteins regulate pathological vs. physiological angiogenesis.

Based on the data from chapters 3 and 4 , we suggest it is now important to determine if autophagy is the mechanism by which capillaries are lost in skeletal muscle regression. This is a new and untested hypothesis. Autophagy is a critical regulatory process in many tissues and proven to be important in skeletal muscle. While autophagy is a known to be an apoptotic trigger, very recent evidence suggesting that TSP-1 mediated autophagy does not trigger apoptosis, yet can still decrease vascularity [1]. While this study was performed in cancer cells, it fits well with our findings. That is, we show no apoptosis or necrosis in the presence of a TSP-1 mimetic, yet a decrease in capillarity (chapters 3 and 4). At present, we are unaware of any studies that examine what role autophagy plays in skeletal muscle angiogenesis. Further still, no data exists on TSP-1's role in autophagy in any tissue beyond that recently reported in the 
context of cancer. While only speculative at this point, autophagy could be a non-apoptotic mechanism for the removal of endothelial cells in skeletal muscle, or reduction in their viability, and hence a resultant shrinking of the capillary network. This is a potentially exciting new role for TSP-1 in skeletal muscle.

One important protein that was not examined in this dissertation is CD47. CD47 is a TSP-1 receptor that has been shown to be a critical component of the TSP-1 anti-angiogenic pathway. However, its role in skeletal muscle is poorly studied. Since CD47 is speculated to be a powerful global regulator of nitric oxide, it certainly could be playing an important role in skeletal muscle, as nitric oxide has a variety of functions that are important to skeletal muscle. The current work established that CD36 is important in skeletal muscle capillary rarefaction (chapters 3 and 4), however it would also be interesting and important to see how manipulation of CD47 affects skeletal muscle structure and function. Indeed, CD47 has been shown to have overlapping functions with CD36, and may work in concert with CD36 to decrease vascularity. If this is true, we may see greater deficits with the manipulation of both CD36 and CD47, versus just CD36 alone. This may also be why we do not see functional differences in muscle shown in chapters 3 and 4.

Finally, our the work showing that TSP-1 is influencing mitochondrial function suggests further work in isolated mitochondria could yield an intriguing role for TSP-1 in skeletal muscle. The role that TSP-1 plays in mitochondrial function remains almost entirely unexplored, with the only work done in mitochondria and TSP-1 relating to biogenesis, and not function. Indeed, if TSP-1 decreases mitochondrial number and/or function, as suggested by our data in chapter 4, it only strengthens the role for TSP-1 in controlling overall muscle function. Our data in chapter 4 is also the first to suggest that this regulation of mitochondria may occur through CD36, and not 
CD47 as previously suggested [2]. Parsing out the pathways that are important in this potential TSP-1 control of mitochondrial number/function will provide important mechanistic understanding on TSP-1 role in muscle function. Also, it would be interesting to determine what differential affects, if any, TSP-1 has on mitochondrial subpopulations in skeletal muscle (subsarcolemmal vs. interfibrillar), as each has been suggested to have distinct and important roles. 


\section{Citations}

1. Kalas, W., et al., Thrombospondin-1 receptor mediates autophagy of RAS-expressing cancer cells and triggers tumour growth inhibition. Anticancer Res, 2013. 33(4): p. 142938.

2. Frazier, E.P., et al., Age-dependent regulation of skeletal muscle mitochondria by the thrombospondin-1 receptor CD47. Matrix Biology, 2011. 


\section{Chapter 8: Appendix}




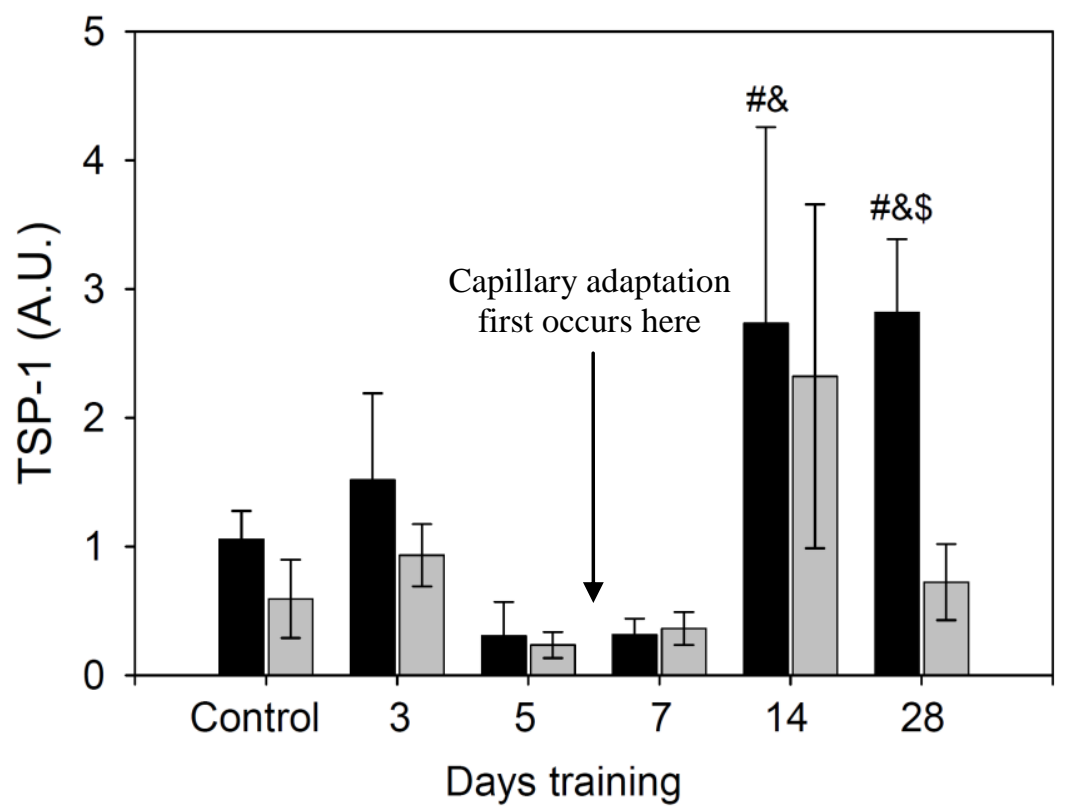

Figure 1: Time course protein expression of TSP-1 following training. Control mice are untrained, all others voluntarily trained using a running wheel for $3,5,7,14$, or 28 days ( $n=12$ /group). Following training, half of the mice per group ( $\mathrm{n}=6$ ) were evaluated under basal conditions (black bars), and the other half $(\mathrm{n}=6)$ performed a single bout of acute exercise ( 1 hour, $20 \mathrm{~m} / \mathrm{min}, 10^{\circ}$ incline) with muscles harvested 4-hours post exercise (gray bars). A.U.= arbitary densitometry units (normalized to GAPDH). Animals from this study showed significant capillary increase (as measured by capillary-to-fiber ratio) by 7 days, which continued to increase through 28 days. \#: Significantly different compared to 14-day trained Basal levels, $p<0.05$; \&: significantly different compared to 28 -day trained Basal levels, $p<0.05$; \$: signifies $\mathrm{p} \leq 0.10$ compared to Basal control. Adapted from Data submitted to The Journal of Physiology (May2013, preliminary acceptance) "Temporal response of positive and negative regulators in response to acute and chronic exercise training in mice” Sara A. Olenich, Navarre Gutierrez-Reed, Gerald N. Audet, and I. Mark Olfert 

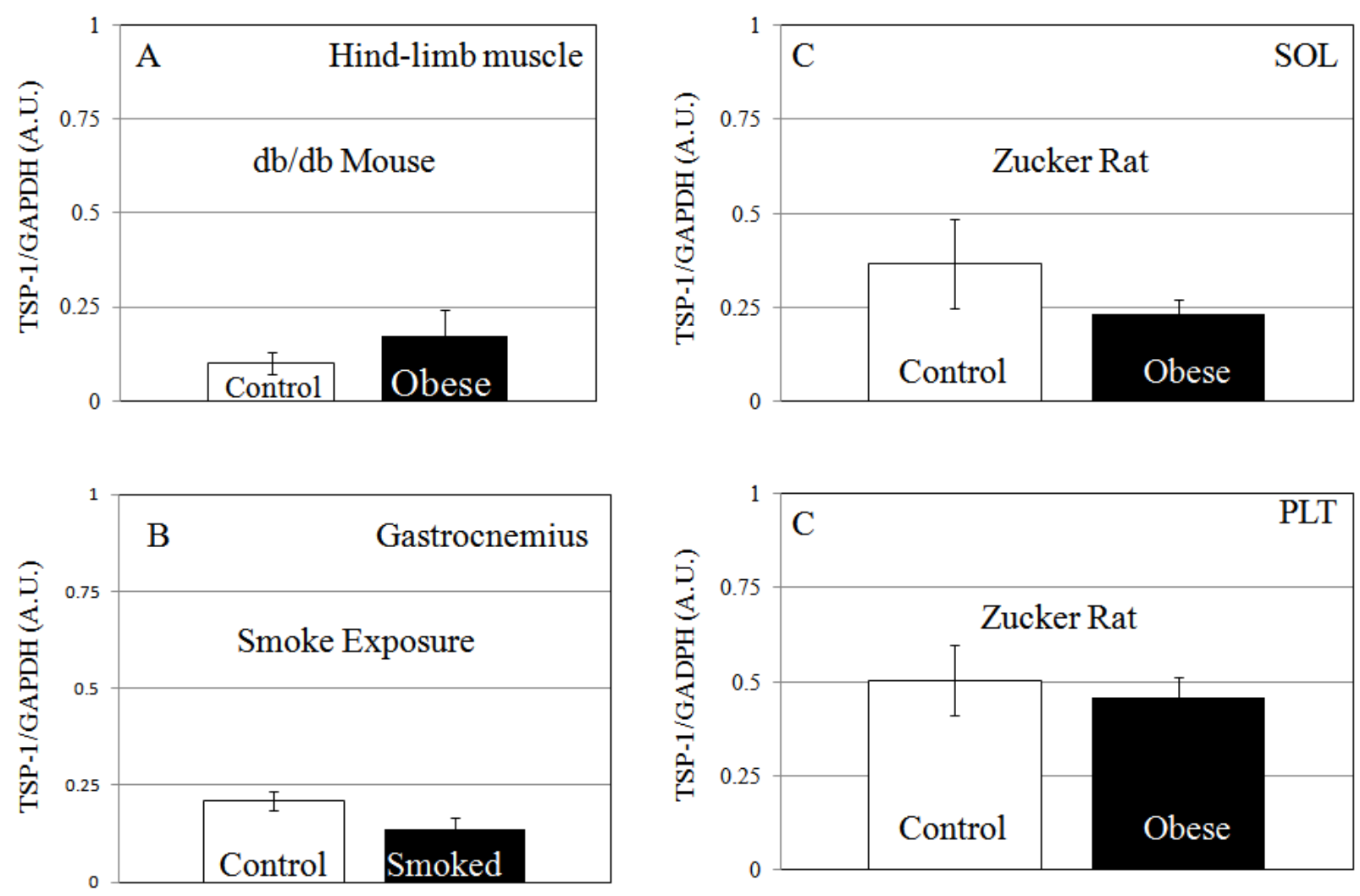

Figure 2: Chronic systemic inflammation does not change skeletal muscle TSP-1 levels in

several different rodent models. Thrombospondin-1 remained unchanged in the A) obese

$\mathrm{db} / \mathrm{db}(\mathrm{N}=4 /$ group$)$ and $\mathrm{B})$ chronic cigarette smoke mouse models ( $\mathrm{N}=8-10 /$ group). The $\mathrm{db} / \mathrm{db}$ mice have $\mathrm{a}$ genetically induced leptin deficiency which results in obesity and increased systemic inflammation. Mice in panel B were subjected to 6 months of direct cigarette smoke exposure. Mice were subjected to 12 cigarettes over 60 minutes, 5 days a week. Cigarette smoke has been shown previously to induce systemic inflammation. C) Obese Zucker rats (N=6/group) also have no increased TSP-1 in two distinct muscles (SOL and PLT). Obese Zucker rats are similar to the $\mathrm{db} / \mathrm{db}$ mice in that they have dysfunctional leptin signaling and grow extremely obese and have been shown to have increased systemic inflammation. Unpublished data 

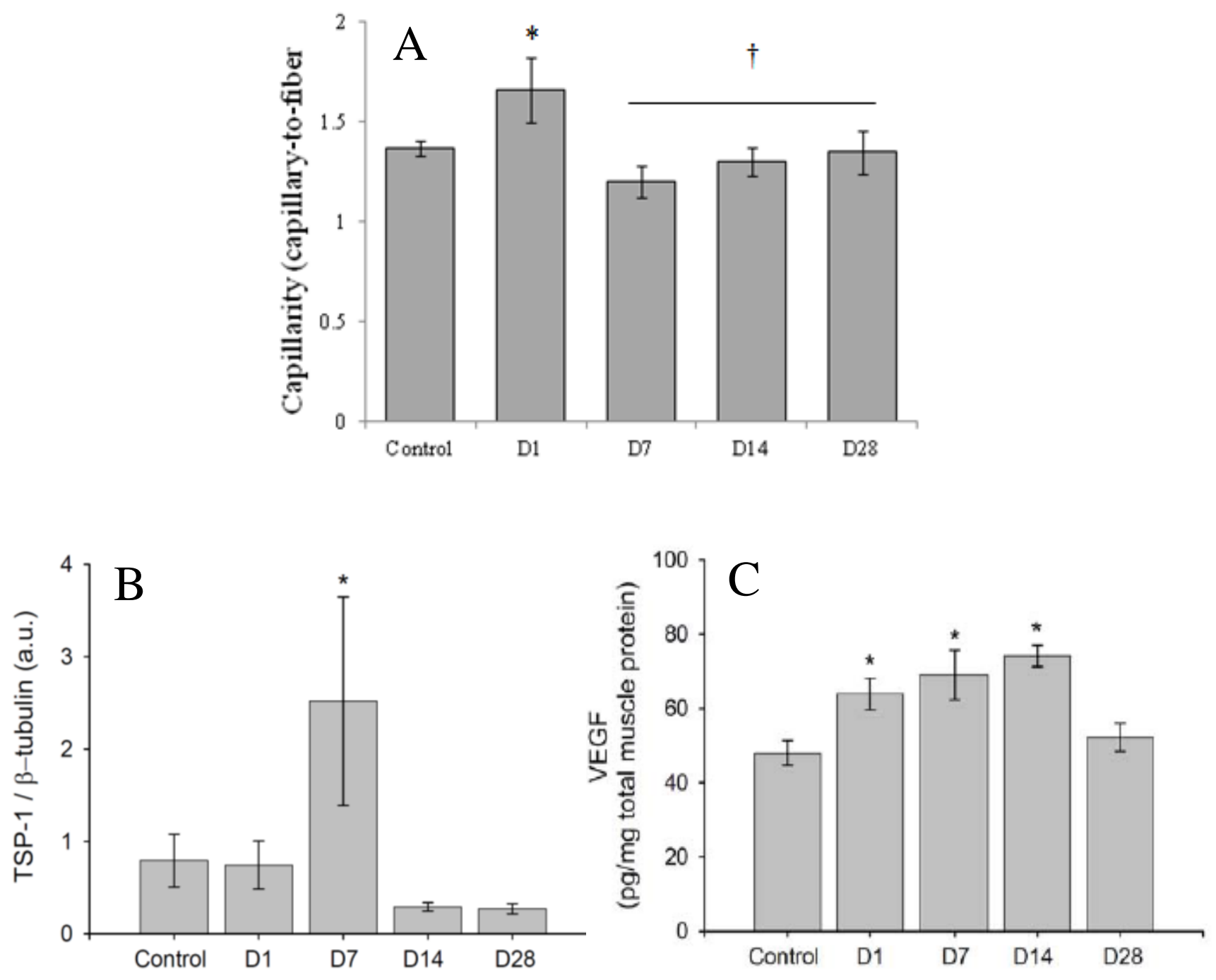

Figure 3: Detraining results in a loss of capillaries, along with increased TSP-1 and VEGF.

Animals underwent 21 days of training where they had free 24 hour access to running wheels. Animals spent apporximately 300-360 minutes per day running (data not shown). A) After 21 days the D1 group was sacrificed, while groups D7-D28 had wheels removed but were allowed free range of their cages for either 7 (D7), 14 (D14), or 28 days (D28). After 21 days of training there was a signfiicant increase in capillarity, as expected. After 7 days without the wheels (i.e. detraining), there was significant drop in capillarity that remained low through 28 days. B) This reduction in capillarity at 7 days coincided with an increase in TSP-1 protein levels. C) Surprisingly, VEGF levels were increased from D1-D14. * $\mathrm{P} \leq 0.05$ from control; $\uparrow \mathrm{P} \leq 0.05$ from D1. Adapted from data to be submitted to The Journal of Physiology (August 2013) "Effects of Detraining on the Temporal Expression of Key Positive and Negative Angioregulatory Proteins in Skeletal Muscle” Sara A. Olenich, Gerald N. Audet, Kathleen A. Roberts, and I. Mark Olfert 


\title{
Chronic Delivery of a Thrombospondin-1 Mimetic Decreases Skeletal Muscle Capillarity in Mice
}

\author{
Gerald N. Audet, Daniel Fulks, Janelle C. Stricker, I. Mark Olfert*
}

Division of Exercise Physiology, Center for Cardiovascular and Respiratory Sciences, West Virginia University School of Medicine, Morgantown, West Virginia, United States of America

\begin{abstract}
Angiogenesis is an essential process for normal skeletal muscle function. There is a growing body of evidence suggesting that thrombospondin-1 (TSP-1), a potent antiangiogenic protein in tumorigenesis, is an important regulator of both physiological and pathological skeletal muscle angiogenesis. We tested the hypothesis that chronic exposure to a TSP-1 mimetic (ABT-510), which targets the CD36 TSP-1 receptor, would decrease skeletal muscle capillarity as well as alter the balance between positive and negative angiogenic proteins under basal conditions. Osmotic minipumps with either ABT510 or vehicle ( $5 \%$ dextrose) were implanted subcutaneously in the subscapular region of C57/BL6 mice for 14 days. When compared to the vehicle treated mice, the ABT-510 group had a $20 \%$ decrease in capillarity in the superficial region of the gastrocnemius (GA), $11 \%$ decrease in the plantaris (PLT), and a 35\% decrease in the soleus (SOL). ABT-510 also decreased muscle protein expression of vascular endothelial growth factor (VEGF) in both the GA $(-140 \%)$ and SOL $(-62 \%)$; however there was no change in VEGF in the PLT. Serum VEGF was not altered in ABT-510 treated animals. Endogenous TSP-1 protein expression in all muscles remained unaltered. Tunnel staining revealed no difference in muscle apoptosis between ABT-510 and vehicle treated groups. These data provide evidence that the anti-angiogenic effects of TSP-1 are mediated, at least in part, via the CD36 receptor. It also suggests that under physiologic conditions the TSP-1/CD36 axis plays a role in regulating basal skeletal muscle microvessel density.
\end{abstract}

Citation: Audet GN, Fulks D, Stricker JC, Olfert IM (2013) Chronic Delivery of a Thrombospondin-1 Mimetic Decreases Skeletal Muscle Capillarity in Mice. PLoS ONE 8(2): e55953. doi:10.1371/journal.pone.0055953

Editor: David D. Roberts, Center for Cancer Research, National Cancer Institute, United States of America

Received August 28, 2012; Accepted January 4, 2013; Published February 6, 2013

Copyright: (c) 2013 Audet et al. This is an open-access article distributed under the terms of the Creative Commons Attribution License, which permits unrestricted use, distribution, and reproduction in any medium, provided the original author and source are credited.

Funding: This project was supported by funding from the National Institutes of Health T32 student training grant NIH5T32HL090610 (GNA) and by the American Heart Association AHA10BGIA3630002 (IMO). The funders had no role in study design, data collection and analysis, decision to publish, or preparation of the manuscript.

Competing Interests: The authors have declared that no competing interests exist.

*E-mail: molfert@hsc.wvu.edu

\section{Introduction}

Physiologic angiogenesis and homeostasis of adult blood vessels is a complex process that is highly regulated by a balance between positive and negative angiogenic proteins. While there is a substantial body of evidence on the role positive angiogenic factors, such as vascular endothelial growth factor (VEGF) [1,2,3], the role of negative angiogenic factors is incomplete and poorly understood [4].

There are a number of known angiogenic inhibitors, of which thrombospondin-1 (TSP-1) is thought to play a prominent role in skeletal muscle angiogenesis [4]. TSP-1 is a large $(450 \mathrm{kD})$ extracellular protein that has a wide array of functions [5]. First discovered for its role in wound healing and platelet activation, it also has important roles in apoptosis, inflammation, nitric oxide signaling, and inhibition of positive angiogenic proteins $[6,7,8,9,10]$. Acting through CD36 (one of its receptors), TSP-1 has been shown to prevent endothelial cell adhesion, growth, and migration, as well as increase apoptosis [11,12,13]. The antiangiogenic effects of TSP-1 in cancer pathology are well established. For example, a reduction in TSP-1 has been shown to increase tumor vessel growth, whereas pharmacological administration of several different TSP-1 mimetics has helped decrease tumor size and disease progression in animal models $[14,15,16,17]$.
Under physiologic conditions, TSP-1 expression has been shown to be responsive to exercise $[18,19]$, which suggests that TSP-1 may play a role in regulating exercise-induced skeletal muscle angiogenesis. TSP-1 KO mice have elevated skeletal muscle capillarity compared to wild-type mice, suggesting a critical role for TSP-1 in the physiological maintenance of capillaries [4]. Evidence in rats also shows that hindlimb unloading increases TSP-1 in association with decreases in skeletal muscle capillarity [3]. Recently, TSP-1 and it's receptor CD47 have also been suggested to play a pivotal role in skeletal muscle mitochondrial biogenesis, and therefore it is likely to be important in overall skeletal muscle function and adaptation to exercise stress [20].

Given the growing data implicating that importance of TSP-1 as a key angiogenic regulator in skeletal muscle, we sought to determine the consequences of chronically stimulating the TSP-1 pathway using ABT-510. ABT-510 is a mimetic of the conserved type I repeat region of TSP-1 which has been shown previously to inhibit angiogenesis through the CD36 receptor [12,13,21]. ABT510 has been synthesized to have a longer half life in circulation than the native type I repeats of TSP-1 which allows for increased chronic stimulation of the TSP-1/CD36 pathway [22]. ABT-510 has been shown to be a potent inhibitor of vascular growth in tumorigenesis both in vitro and in vivo [15,23,24,25,26,27,28], which has been attributed to its binding to the CD36 receptor. Using this peptide we aimed to examine whether increased 
stimulation of the CD36 arm of the TSP-1 pathway affected skeletal muscle structure and function. Given that TSP-1 is a negative angiogenic regulator, we hypothesized that mice subjected to the ABT-510 would have lower skeletal muscle capillarity, mirrored by an altered balance between positive and negative angiogenic proteins.

\section{Methods}

\section{Animals}

This study used 10-12 week old male C57BL/6 mice purchased from Jackson Laboratories (Strain no. 000664, C57BL/6J, Bar Harbor, ME). Mice were randomly selected to be placed in two groups (control, $\mathrm{n}=8$; experimental $\mathrm{n}=8$ ). All procedures that involved animals were approved by the West Virginia University Institutional Animal Care and Use Committee.

\section{Exercise Testing Protocol}

Whole body exercise capacity was assessed using a graded maximal exercise test (max test) 48 hours before pump implantation and then again 48 hours before muscle harvest (12 days after implantation). This was done to ensure that the exercise bout did not influence protein levels in muscles harvested on the final study day (Day 14) [18]. Maximal run test procedure: mice were given a 5 minute warm-up (speed $<4 \mathrm{~m} / \mathrm{min}$ ) at a 10 degree incline on a commercially available rodent treadmill (Columbus Instruments, Columbus, $\mathrm{OH})$. After the warm-up period, the treadmill was increased $2 \mathrm{~m} / \mathrm{min}$ every 30 seconds until the animals reached exhaustion. Exhaustion was defined by a single observer as the inability of the animal to stay on the treadmill at a given speed, and/or more than 3 seconds spent on the shock grid. Animals were encouraged to run as fast and as long as possible by using forced air and a weak electrical deterrence. Final run time and velocity were recorded and compared between groups.

\section{Osmotic Pump Drug Delivery}

The TSP-1 mimetic ABT-510 was generously provided by Abbott (Abbott, IL). This compound was chosen for use in this study based on previous and preliminary work showing its effectiveness in decreasing capillarity in tumors, both in vitro and in vivo $[15,22,23,24,25,29,30]$. ABT-510 was dissolved in 5\% dextrose (vehicle) over a 48 hour period to ensure the compound was fully dissolved, and then inserted into mini-osmotic pumps (Model 1002, Alzet Osmotic Pumps, Cupertino, CA,). Pumps were filled with either the drug or vehicle solution 24 hours in advance of implantation as per manufacture instructions. We delivered the maximum concentration of $30 \mathrm{mg} / \mathrm{kg} /$ day based on the maximum pump capacity $(100 \mu \mathrm{L})$, the mass of the animals, and the solubility of the drug (ABT-510 solubility curves [22]). Pumps were surgically inserted (flow-moderator first) subcutaneously in the scapular region while the mice were under anesthesia $(2 \%$ isoflurane). The surgical site was disinfected with iodine and closed using sutures. Animals were then housed individually and observed daily for the remainder of the study for pain or distress. Pumps remained in the animals for 14 days, after which they were sacrificed and tissue/organ samples were collected and stored at $-80^{\circ}$ for later analysis.

\section{Morphometry}

Hindlimb skeletal muscle was surgically excised and frozen in isopentane cooled by liquid nitrogen. Frozen tissue was cut using a $-20^{\circ} \mathrm{C}$ cyromicrotome (Jung-Reichert Cryocut 1800: Cambridge Instruments, Germany) to yield $10 \mu \mathrm{m}$ transverse sections. Great care was taken to ensure the cryosectioned muscles were cut along the transverse plane. Sections were stained for dipeptidly-peptidase IV (DPP IV) and alkaline phosphatase (AP) following the method of Lojda (1979), as applied to skeletal muscle tissue [31,32]. A light microscope (Zeiss primo star, Zeiss, Oberkochen, Germany) was used to digitally acquire (Axiocam IC c3, Axiovision 4.8.2.0, Zeiss, Oberkochen, Germany) $20 \times$ images of the gastrocnemius, plantaris, and soleus. Capillary and myofiber counting was performed by a single individual blinded to group identification. For the gastrocnemius muscle, we obtained images in a checkerboard fashion across the entire muscle, thus both superficial and deep regions within the gastrocnemius could be included in the analysis. For the plantaris and soleus muscles, respectively, the entire muscle was imaged and analyzed. Counting was performed by visualization from acquired images using a custom program in MATLAB (version 7.0.0.27, The Mathworks, MA, USA) allowing the operator to visually mark and count the capillaries and fibers on each image. Capillary-to-fiber ratio $(\mathrm{C}: \mathrm{F}$, number of capillaries/number muscle fibers), capillary density (CD, number of capillaries $/ \mathrm{mm}^{2}$ muscle fiber area), and fiber cross sectional area (FCSA) were separately calculated for the gastrocnemius (GA), soleus (SOL), and plantaris (PLT) $(\mathrm{n}=33-$ $101 \mathrm{images} /$ muscle/group).

\section{Protein Analysis}

GA, SOL, and PLT muscles from each group were excised and flash frozen in liquid nitrogen. They were then separately homogenized in a lysis buffer containing $50 \mathrm{mM}$ Tris/HCl (pH 7.4), $150 \mathrm{mM} \mathrm{NaCl,} 0.5 \%$ Triton X-100, and protease inhibitors (Complete Tablet, Roche Applied Science, Indianapolis, IN). Homogenates were centrifuged at $4^{\circ} \mathrm{C}$, at $8,000 \mathrm{~g}$ for 10 minutes, and supernatants removed and placed in new tubes. Blood samples were obtained from the heart and allowed to coagulate on ice. They were then centrifuged at $3000 \mathrm{~g}$ for 10 minutes and flash frozen in liquid nitrogen. Total protein was measured by bradford assay (\#23236 Pierce Coomassie Plus Protein Assay Kit, Thermo Scientific, Rockford, IL).

Quantification of VEGF was made from a total of $100 \mu \mathrm{g}$ of protein using a commercially available ELISA kit according to the manufactures instructions (\# MMV00, R\&D Systems, Minneapolis, MN, USA). Quantification of VEGFR-2 and P-VEGFR-2 were made from a total of $100 \mu \mathrm{g}$ of protein using a commercially available ELISA kit according to the manufactures instructions (\# 7335S, \#7340S, Cell Signaling, Danvers, MA, USA). TSP-1 and CD36 were analyzed via western blot. In brief, samples were separated on a 3-8\% SDS-PAGE (NuPAGE Novex 3-8\% TrisAcetate Midi Gel, Invitrogen, Burlington, ON, Canada) and blotted onto a $0.45 \mu \mathrm{m}$ nitrocellulose membrane (Pierce nitrocellulose membrane, Thermo Scientific, Rockford, IL). After blocking with $5 \%$ fat-free milk, membranes were probed using antibodies against TSP-1 (1:1000, clone A6.1, \#399300, Invitrogen, Burlington, ON, Canada), $\beta$-tubulin (1:1000, \#2148, Cell Signaling), CD36 (1:250 \#552544, BD Pharmingen, Franklin Lakes, NJ, USA), secondary HRP-conjugated anti-mouse (1:1000, \#p0260, Dako, GE Healthcare, Piscataway, NJ) and secondary HRP-conjugated anti-rabbit (1:1000, \#p0217, Dako). Proteins were visualized using chemiluminescence detection (Pierce ECL, Thermo Scientific, Rockford, IL) and digitally imaged (G:BOX Gel imager, Syngene, Cambridge, UK) using Genesnap software (Ver. 7.01, Syngene, Cambridge, UK). Equal protein loading was verified by immunodetection of $\beta$-tubulin as our loading control. Quantification of protein expression levels were carried out using $\mathrm{NIH}$ Image J Software (v1.62) and expressed as densitometric arbitrary units (AU). 


\section{Apoptosis}

Nuclei exhibiting apoptotic changes were identified by TdTmediated dUTP nick end labeling (TUNEL) according to the manufacturer's recommendations (Roche Molecular Biochemicals, Pleasanton, CA). Briefly, muscle cross sections were cut on a cryostat $(10 \mu \mathrm{m})$ and fixed in $4 \%$ paraformaldehyde at room temperature, blocked in $3 \% \mathrm{H} 2 \mathrm{O} 2$ in $100 \%$ methanol at room temperature, and permeabilized in $0.1 \%$ Triton $\mathrm{X}$ and $0.1 \%$ sodium citrate. TUNEL reaction mix was added in a 1:7.5 dilution, and the sections were incubated at $37^{\circ} \mathrm{C}$ for $1 \mathrm{~h}$. Sections were reacted with fluorescein antibody for $30 \mathrm{~min}$ at $37^{\circ} \mathrm{C}$, and substrate was added for color development. To control for falsepositives, samples were then counter stained by DAPI staining to identify the nucleus. TUNEL staining was performed using a fluorescein TUNEL kit at 1:7.5 dilution as recommended by the manufacturer (Roche Molecular Biochemicals, Pleasanton, CA). Positive nuclei were counted, and at high power $(\times 400)$, it was determined whether they were associated with the myofiber or with the interstitial space. The number of positive nuclei is expressed per whole muscle section. Positive controls were created using a DNase treatment of 1 serial section (\# AM1906, DNA-free, Ambion, Austin, TX). Negative controls were created by staining 1 serial section only for DAPI without TUNEL staining. This resulted in each sample containing 1 negative, 1 positive, and 2 experimental sections per slide.

\section{Statistics}

All data are presented as means+/-SEM. To examine body mass, organ masses, muscle capillarity and molecular responses we used a student's T-test. A repeated measures ANOVA was used to analyze the maximal running test. An alpha level at $P<0.05$ was selected for statistical significance.

\section{Results}

\section{Exercise Testing and Morphometry}

Body and Muscle Mass. There was no significant difference in the absolute body or individual hindlimb muscle masses between ABT-510 (mimetic) and vehicle treated groups (Table 1). Heart and skeletal muscle normalized to body mass were not different between the groups (Table 1).

Maximal Running Test. There was no difference in maximal running speed between vehicle and mimetic treated animals pre- or post-treatment.

Table 1. Muscle and Body Masses.

\begin{tabular}{lll}
\hline & Vehicle & ABT-510 \\
\hline & $27.5 \pm 1.23$ & $27.1 \pm 0.87$ \\
\hline Body Mass (g) & $136.8 \pm 4.56$ & $140.8 \pm 4.09$ \\
GA (mg) & $19.7 \pm 0.90$ & $19.0 \pm 0.53$ \\
PLT (mg) & $7.7 \pm 0.47$ & $7.8 \pm .048$ \\
SOL (mg) & $123.5 \pm 5.76$ & $134.3 \pm 5.82$ \\
HRT (mg) & $5.0 \pm 0.07$ & $5.2 \pm 0.12$ \\
GA/BM (mg/g) & $0.72 \pm 0.04$ & $0.70 \pm 0.02$ \\
PLT/BM (mg/g) & $0.29 \pm 0.01$ & $0.28 \pm 0.01$ \\
SOL/BM (mg/g) & $4.4 \pm 0.26$ & $5.0 \pm 0.17$ \\
HRT/BM (mg/g) & & \\
\hline Values: Mean \pm Standard Error. & \\
*P=0.05. \\
doi:10.1371/journal.pone.0055953.t001
\end{tabular}

Morphometry. In the superficial region of the gastrocnemius (GA) muscle there was a $20 \%$ decrease in $\mathrm{C}: \mathrm{F}(\mathrm{P} \leq 0.05)$ and a similar trend in $\mathrm{CD}(25 \%$ decrease, $\mathrm{P}=0.055)$ in the mimetic group compared to vehicle (Figure $1 \& 2$ ). There was no statistical difference in $\mathrm{C}: \mathrm{F}$ of the deep portion of the GA between groups, however there was a significant $25 \%$ decrease in $\mathrm{CD}(\mathrm{P} \leq 0.05)$. FCSA was not significantly different between groups in either portions of the GA.

In the plantaris (PLT) muscle, there was a $11 \%$ decrease in the $\mathrm{C}: \mathrm{F}$ in the PLT in the mimetic group compared to vehicle $(\mathrm{P} \leq 0.05)$ (Figure 3). There was no difference in CD or FCSA in the PLT.

In the soleus (SOL) muscle, there was a $35 \%$ decrease in the $\mathrm{C}: \mathrm{F}$ in the SOL in the mimetic group compared to vehicle $(\mathrm{P} \leq 0.01)$ (Figure 3). There was no difference in $\mathrm{CD}(\mathrm{P}=0.08)$ or FCSA $(\mathrm{P}=0.09)$.

\section{Endogenous VEGF, VEGFR-2, p-VEGFR-2, TSP-1, and CD36 Levels}

Skeletal muscle VEGF protein expression was decreased by $147 \%$ and $62 \%$ in the GA and SOL respectively $(\mathrm{P} \leq 0.05$, Figure 4). In contrast, no significant change in VEGF protein expression was seen in the in the PLT. Serum VEGF levels were unchanged between ABT-510 and vehicle treated animals.

Total VEGFR-2 and phorphylated-VEGFR-2 (p-VEGFR-2) levels were also assessed in the GA. There was no difference in total VEGFR-2 levels, p-VEGFR-2 levels, or a ratio of VEGFR2/p-VEGF-R2 between ABT-510 and vehicle treated mice (data not shown).

Endogenous TSP-1. protein expression of endogenous TSP1 was not significantly different in any of the muscles analyzed (SOL, PLT, GA) between the treated groups (data not shown).

CD36 levels. There was no difference in the receptor levels of CD36 in the GA as assessed by western blot (data not shown).

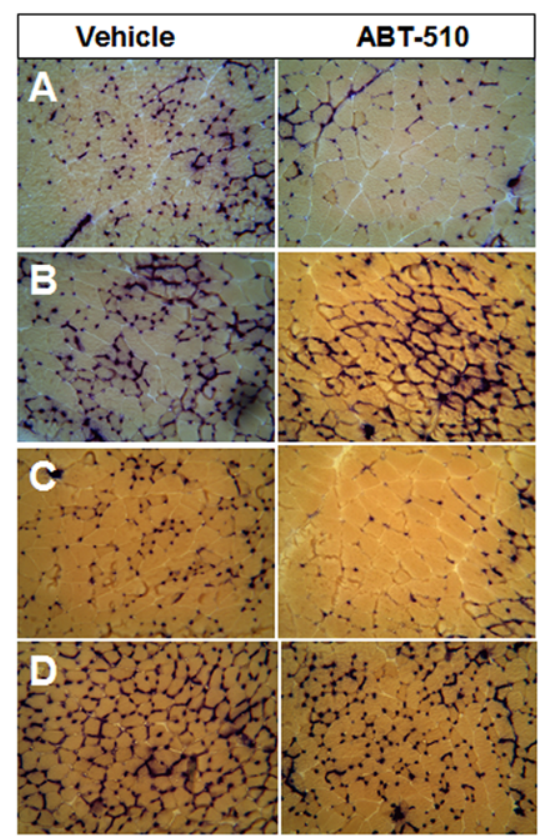

Figure 1. Representative figures of the histology sections. A) Superficial Gastrocnemius Muscle B) Deep Gastrocnemius Muscle C) Plantaris Muscle, D) Soleus Muscle. doi:10.1371/journal.pone.0055953.g001 


\section{Superficial GA}

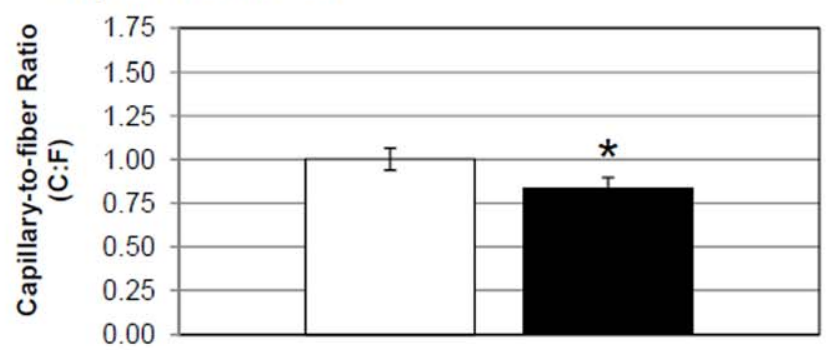

Superficial GA

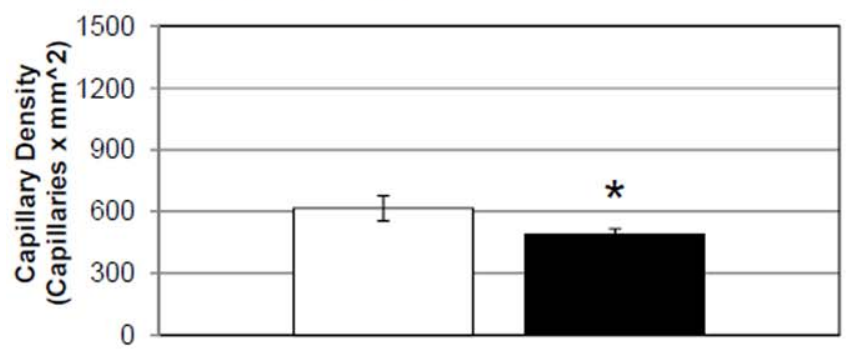

\section{Superficial GA}

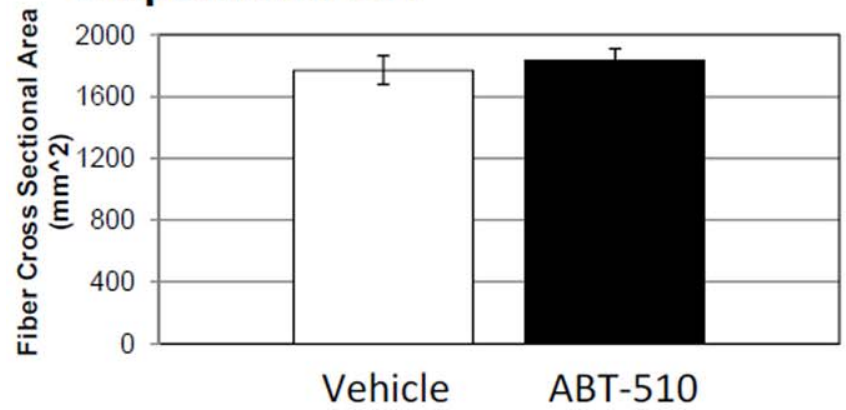

Deep GA

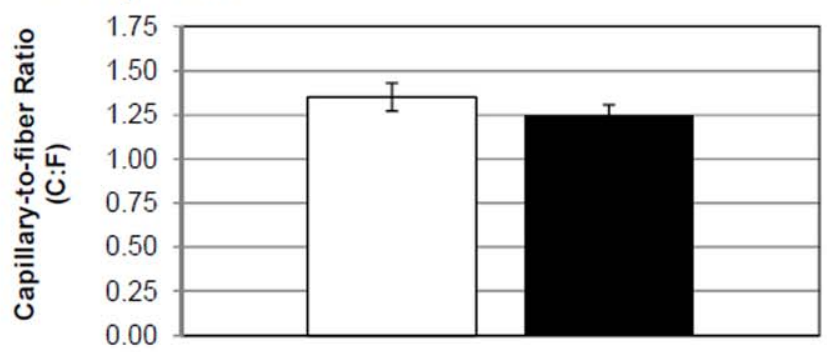

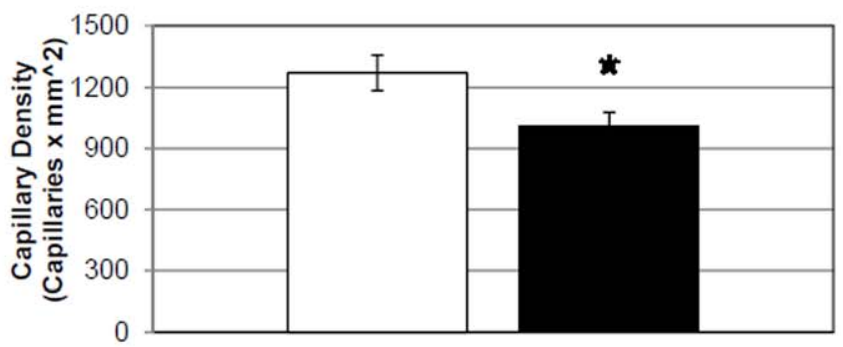

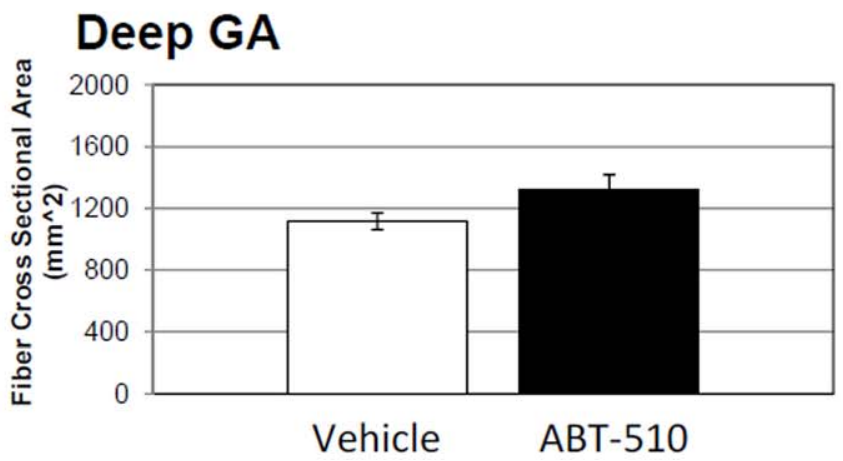

Figure 2. Superficial gastrocnemius capillarity, but not deep, is decreased in the ABT-510 group. Chronic administration of ABT-510 $30 \mathrm{mg} / \mathrm{kg} /$ day decreased capillary to fiber ratio (C:F) and capillary density (CD) in the superficial gastrocnemius (GA). Despite a similar trend, no significant changes in capillarity were seen in the deep GA. There was no change in fiber cross sectional area in either the deep or superficial portions of the $\mathrm{GA} .{ }^{*}=\mathrm{P} \leq 0.05$.

doi:10.1371/journal.pone.0055953.g002

\section{Apoptosis}

Apoptosis was assessed using TUNEL staining for fragmented DNA. There was no difference in the number of TUNEL positive nuclei in any of the muscles between the two treated groups (Figure 5).

\section{Discussion}

The main finding of this study is that chronic exposure to the TSP-1 mimetic ABT-510 significantly decreases skeletal muscle capillarity (Figure 1). To our knowledge, these are the first data to show that chronic stimulation of the TSP-1/CD36 pathway in healthy mammals results in decreased skeletal muscle capillarity. These data are consistent with the anti-angiogenic function of TSP-1 and, more importantly, support the notion that TSP-1 through its CD36 receptor is a critical regulator of skeletal muscle capillarity under physiologic conditions. Indeed, when coupled with the previous observation that loss of TSP-1 increases skeletal muscle capillarity (25) these data provide evidence that the actions of TSP-1 directly influence homeostatic maintenance and/or development of skeletal muscle microvessels.

Skeletal muscle capillarity is decreased in mimetic treated mice

TSP-1 has been shown previously to be important in maintaining skeletal muscle capillarity, where TSP-1 KO mice have approximately 2 fold the number of capillaries as WT controls [33]. Further, TSP-1 has been shown to be important in maintaining capillarity with hindlimb unloading [34]. In this manuscript we build upon this small body of evidence for the importance of TSP-1 in the context of the basal regulation of angiogenesis.

The actions of TSP-1 are complex and multifunctional, due in large part to the diversity of receptors it binds, such as lipoprotein receptor-related protein 1 (LRP-1), CD47, and CD36 [11,35]. Our use of this the TSP-1 mimetic ABT-510, which is a potent CD36 binding peptide, resulted in a decrease in capillarity across three different distinct skeletal muscles. This suggests that TSP-1s 


\section{PLT}

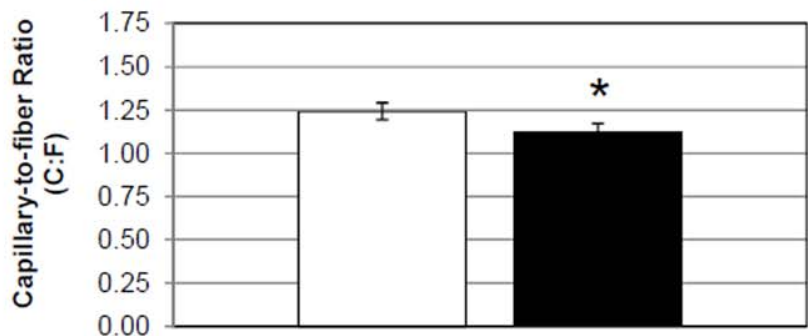

\section{PLT}

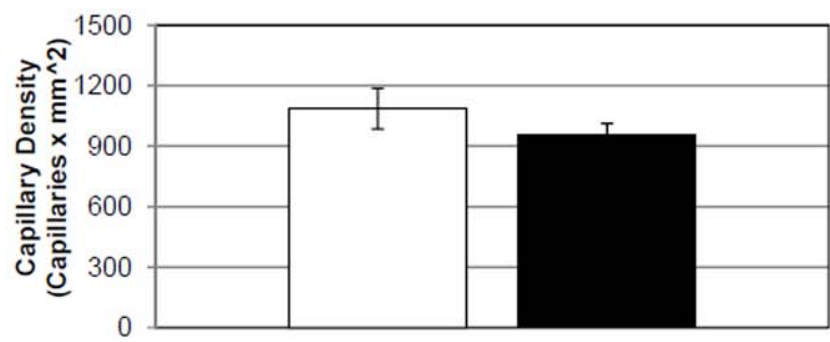

\section{PLT}

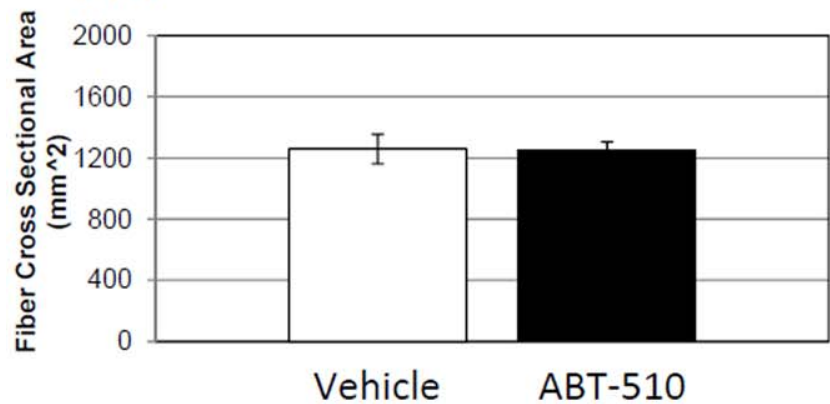

SOL

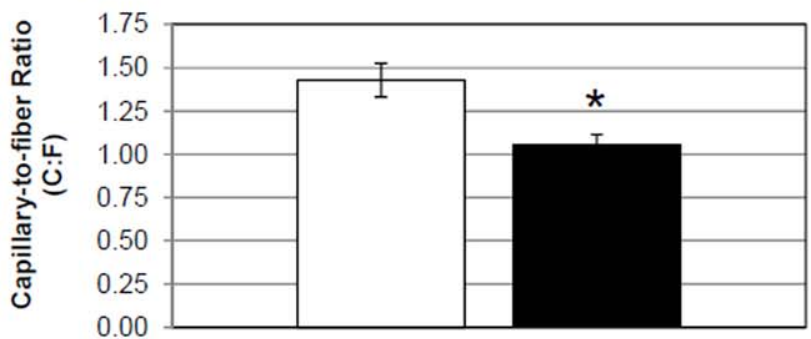

\section{SOL}
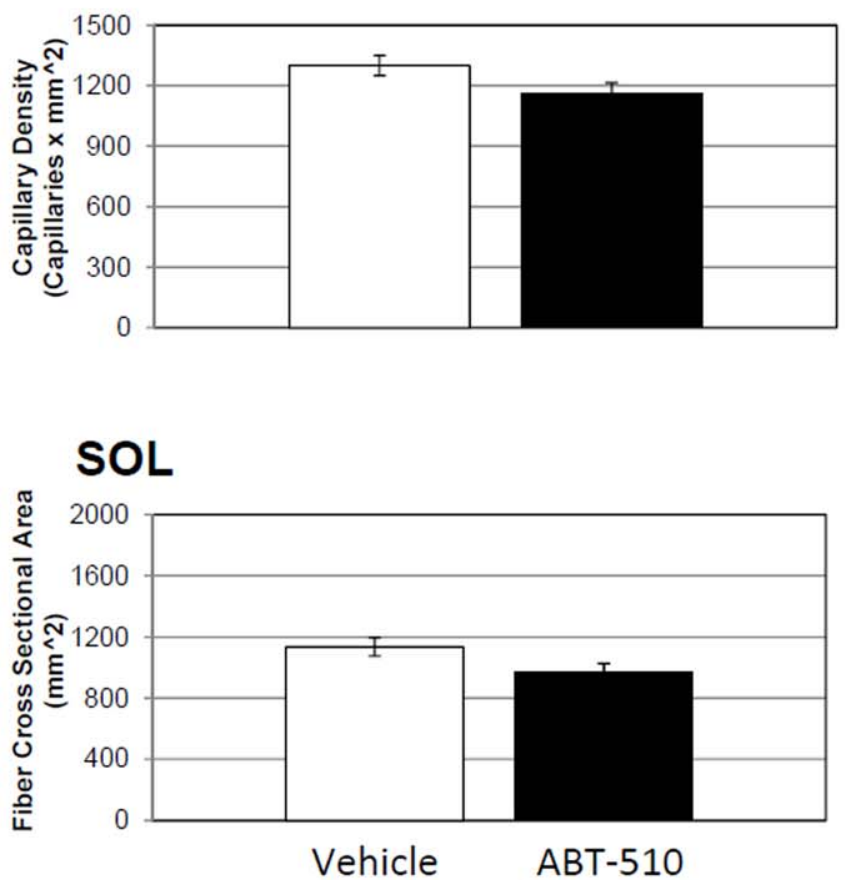

Figure 3. Plantaris and soleus capillarity is decreased in the ABT-510 group. Chronic administration of ABT-510 $30 \mathrm{mg} / \mathrm{kg} / \mathrm{day}$ decreased capillary to fiber ratio (C:F) in the plantaris muscle (PLT) but did not decrease capillary density (CD). ABT-510 decreased capillary to fiber ratio (C:F) and capillary density (CD) in the soleus (SOL). There was no change in fiber cross sectional area in either the PLT or SOL muscle. ${ }^{*}=\mathrm{P} \leq 0.05$. doi:10.1371/journal.pone.0055953.g003

anti-angiogenic properties in skeletal muscle involves a CD36 mediated mechanism. Indeed, this is consistent with the physiological activity of CD36 where TSP-1 binding to CD36 has been shown to be anti-proliferative, anti-angiogenic, and pro-apoptotic [12]. The importance of the TSP1/CD36 pathway has been proven in tumorigenesis, where down regulation of TSP-1 and its binding to CD36 results in a pro-tumorigenesis environment and increase in tumor size $[14,36,37,38]$. Here we show similar actions in skeletal muscle under physiological conditions. This suggests a putative role for TSP-1 in decreasing vascularity across multiple tissues, and not just in tumors.

Given these findings, it is tempting to speculate that elevated skeletal muscle TSP-1 may serve as a biomarker for skeletal muscle dysfunction associated with several chronic conditions known to result in muscle capillary rarefaction, such as that found in diabetes [39,40], and chronic heart and lung disease [41]. It may even be that therapeutic interventions to limit basal TSP-1 expression and/or reduce circulating levels of TSP-1 could be clinically exploited to attenuate the decrements in skeletal muscle function often accompanying these diseases.

\section{Chronic stimulation of the TSP1/CD36 pathway decreases VEGF}

It has been shown previously that TSP-1 can counter the effects of VEGF by multiple mechanisms; including interacting directly with VEGF protein and disrupting its actions at the receptor level $[42,43,44,45,46]$. For example, TSP-1 has been suggested to bind VEGF via its type I repeat region (or 3TSR). Once bound the protein heterodimer is internalized by the scavenger receptor low density lipoprotein receptor-related protein 1 (LRP-1). Here, we show that a chronic administration of ABT-510 (a TSP-1 type I repeat mimetic), results in a decrease in total VEGF protein in both the gastrocnemius muscle and the soleus muscle, along with the decrease in capillarity. It is unclear why a similar decrease in VEGF protein was not seen in the PLT which also showed decreased capillarity, nevertheless these data support previous 
Gastrocnemius

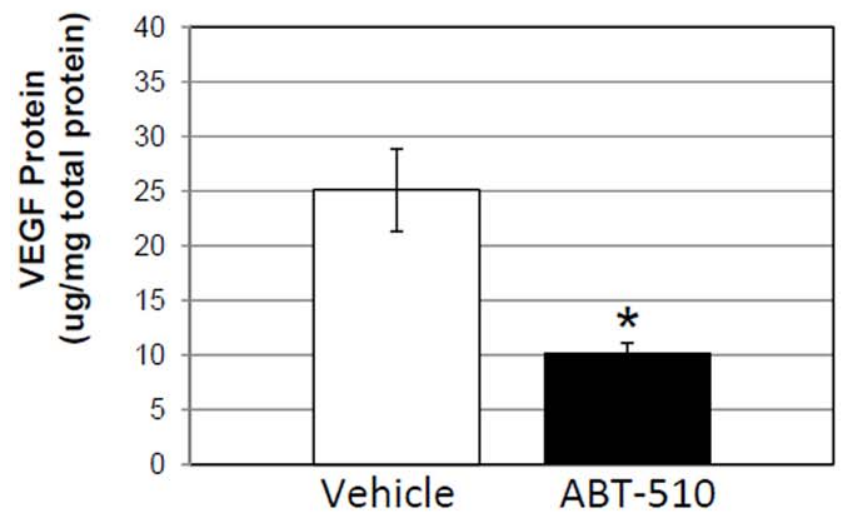

Soleus

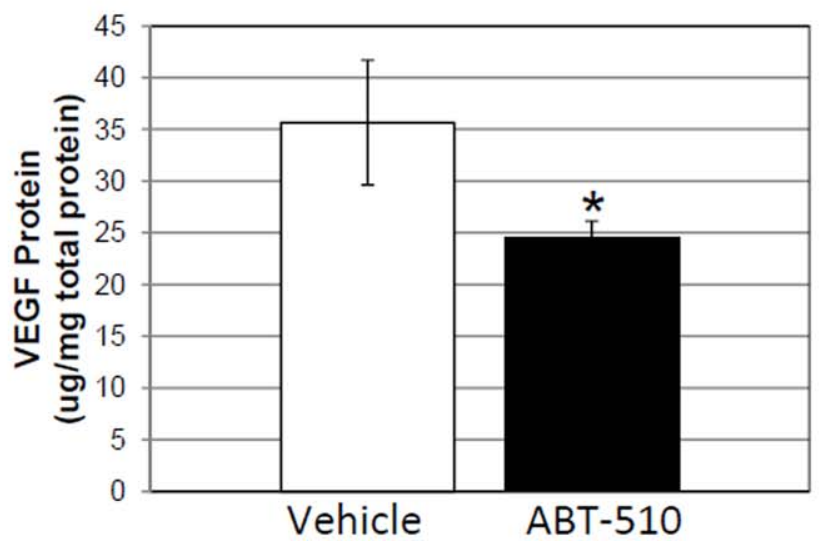

Plantaris

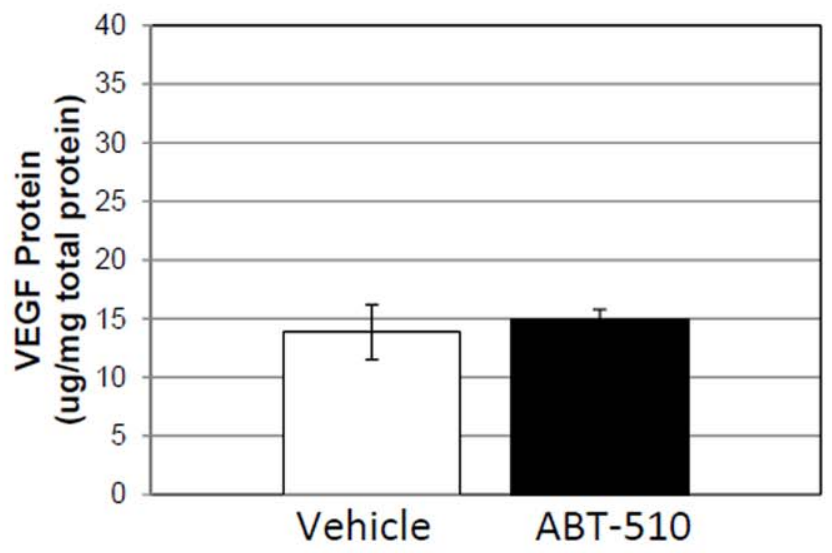

Serum

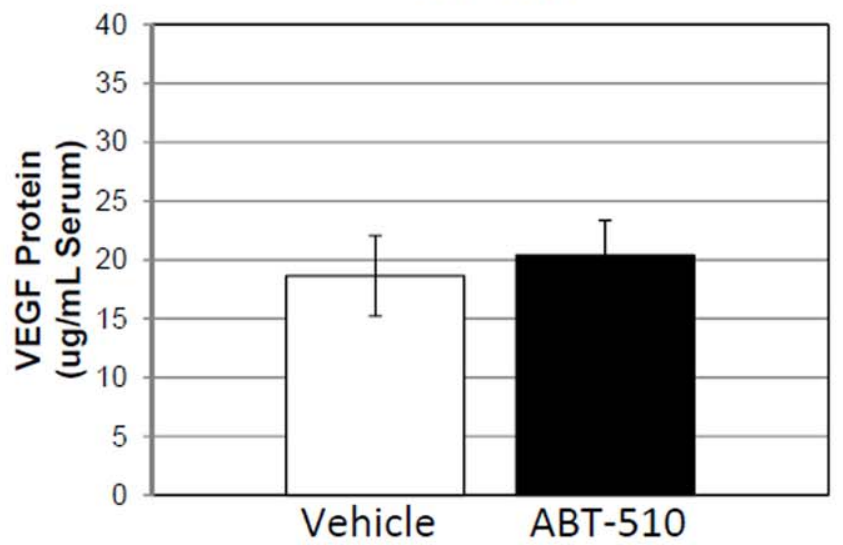

Figure 4. Skeletal muscle VEGF protein content is decreased in the ABT-510 group, but not serum levels. Chronic administration of the mimetic (ABT-510 $30 \mathrm{mg} / \mathrm{kg} /$ day) decreased VEGF protein in the GA, and SOL muscles of C57/BLK6 mice. There was no change in the PLT muscle or in Serum levels. ${ }^{*}=\mathrm{P} \leq 0.05$.

doi:10.1371/journal.pone.0055953.g004

studies suggesting that TSP-1 may be binding and sequestering VEGF - resulting eventually in internalization and biological inactivation $[42,43,44,45,46]$. Explanations for the reduction in VEGF may also involve suppression of VEGF production and/or VEGF signaling. For example, there is a growing body of evidence showing that the internalization of the VEGF/VEGFR-2 complex by endocytosis results in downstream activation of several different signaling pathways [47]. There is also evidence that both CD36 and CD47 can associate with VEGFR-2, and when TSP-1 is present this association can prevent the VEGF ligand binding to its receptor, as well as inducing receptor dephosphorylation and preventing dimerization $[13,21,48,49]$. In this way, TSP-1 could prevent activation and/or endocytosis of the VEGF receptor, blocking yet another biologically active arm of VEGF/VEGF-R complex. Regardless of the mechanism, our data supports the notion that TSP-1 likely plays a regulatory role involving VEGF, thereby enhancing its already potent inherent anti-angiogenic capacity.

In endothelial cells, TSP-1 can also disrupt VEGF actions at the receptor level by dephosphorylating the important receptor VEGFR-2 (Flk-1) [50]. TSP-1 can disrupt VEGFR-2 activation by binding the CLESH region of CD36, triggering a cascade of events which results in direct binding of VEGFR-2, and a resultant decrease in tyrosine phosphorylation of VEGFR-2 [12,13,21]. This includes dephosphorylating the important tyrosine 1175 region; which is critical to initiate downstream VEGF signaling. While it has also been shown previously that ABT- 510 significantly decreases the amount and phosphorylation status of VEGFR-2 in endothelial cells in vitro [24,51], in vivo we found no significant change in the phosphorylation levels of tyrosine 1175 of VEGFR2. Given that the mimetic is a type I repeat, and that total VEGF protein levels were decreased, we were surprised that VEGFR-2 phosphorylation was also not decreased. But, because we only have one time point, it could be that levels of phosphorylation were lower earlier in the chronic dosage but by the end of the study returned to normal levels. While ABT-510 has been shown to decrease VEGFR-2 phosphorylation in tumors [24], it may be that this response is different in skeletal muscle under physiological conditions. It could also be that phosphorylation status in vivo is not lowered by endogenous TSP-1 (or the addition of a mimetic), rather TSP-1 limits or prevents an increase in phosphorylation levels with a VEGF stimulus. This is yet to be elucidated.

Administration of ABT-510 did not significantly alter endogenous levels of TSP-1 in skeletal muscle, indicating that there was no feedback response from the mimetic binding to CD36 to alter endogenous TSP-1 levels in skeletal muscle. However, the 

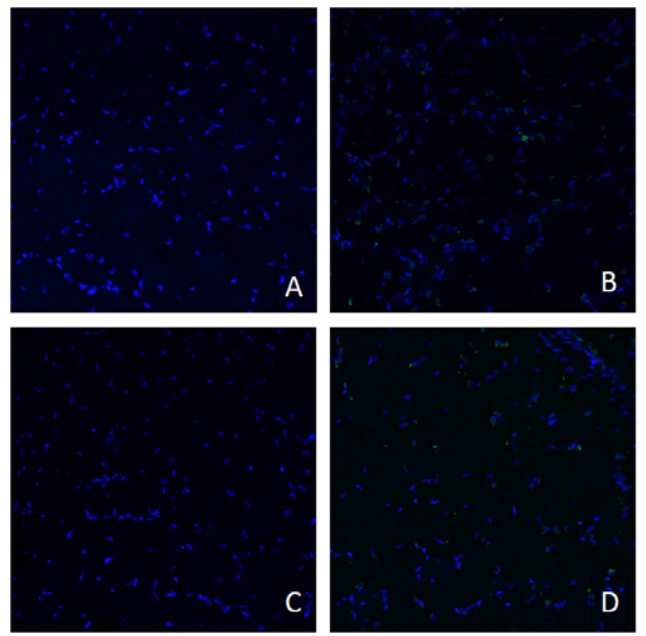

Figure 5. There is no difference in skeletal muscle apoptosis between the mimetic and control groups. TUNEL staining shows that chronic administration of the mimetic (ABT-510 $30 \mathrm{mg} / \mathrm{kg} / \mathrm{day}$ ) did not increase or decrease skeletal muscle apoptosis in any of the muscles tested. Representative GA samples, PLT and SOL data not shown. A) Representative mimetic GA B) Representative positive control mimetic GA C) Representative control GA D) Representative positive control vehicle $G A$.

doi:10.1371/journal.pone.0055953.g005

reduction of skeletal muscle VEGF protein levels in association with the mimetic adds support to the idea that physiologic angiogenesis is controlled by a balance between both positive and negative angiogenic factors, particularly VEGF and TSP-1. It would have been interesting to deliver a higher dose of ABT-510 (>30 ug/kg/day) which may have resulted in greater reductions in muscle VEGF and capillarity; however the dose used was the maximum we could deliver using the small pumps suitable for mice and the 2-week duration we sought. Although shorter duration would allow greater concentrations of drug delivery, we opted to deliver the drug for 14 days to ensure that vascular remodeling would have sufficient time to occur. It could be hypothesized that tipping the scale towards an anti-angiogenic state (stimulated by the presence of ABT-510) would result in a response of positive angiogenic factors to counter this challenge. However, at least with respect to VEGF this was not the case. A decrease in VEGF and no change in endogenous TSP-1 suggests that the muscle has been pushed towards an anti-angiogenic phenotype.

Regardless of mechanism, these data show that the altered angiogenic state of the skeletal muscle can be driven by an increase in a TSP-1 mimetic. The resulting decrease in capillarity establishes the importance of TSP-1/CD36 pathway in maintaining and regulating basal skeletal muscle microvessel density.

\section{Skeletal muscle and endothelial apoptotic levels do not differ between groups}

In addition to its anti-angiogenic properties, TSP-1 is also been known to promote apoptosis [6,52,53,54,55,56,57,58]. TSP-1 is known to induce apoptosis through the CD36 pathway, as shown in previous studies, including those using ABT-510 [5,24,59]. Endothelial apoptosis is a natural mechanism by which capillary regression occurs $[60,61,62,63]$, and has been shown to be central in muscle atrophy during some pathologies, aging, and disuse $[64,65,66,67,68]$. Knowing this, we performed TUNEL staining to look at end stage apoptosis (DNA fragmentation) in all three of the excised muscles. Surprisingly, we found no evidence of apoptosis in any of the muscles, either myocyte or endothelial. The reason for this remains unclear. This is, however, the first in vivo report of ABT-510s affects on skeletal muscle and it could be that ABT-510 is not sufficient to induce myocyte apoptosis in skeletal muscle under these conditions. Indeed, much of the work with TSP-1 and apoptosis pertains to cancer models (e.g. tumorigenesis), endothelial cells, and is typically done in vitro. It is possible that TSP-1 is not as potent a pro-apoptotic protein in skeletal muscle as that seen in endothelial cells or tumors. This is supported by the finding that there was no change in muscle fiber cross-sectional area (Figures 2 and 3). Whether or not the ABT-510, or the TSP-1/CD36 axis, alters in vivo skeletal muscle myocyte apoptosis will require further study.

However, this does not explain why we saw no endothelial cell apoptosis. As mentioned previously, our study only examines one time point. It is possible that apoptosis occurred at an earlier time point than our assessment at the 14 day time point. Indeed, it has been shown previously that hindlimb unloading results in capillary loss in as little as 5 days [34]. If changes in endothelial cell number, and therefore total vasculature, are occurring by 5-7 days it may not be surprising that evidence of apoptosis has been lost by the end of the study (14 days). That is, perhaps apoptosis has already occurred, the cells cleared from the tissue, and the architecture of the vascular network at 14 days has already returned to a new steady state condition absent of apoptotic nuclei. We have yet to elucidate this and more experimentation is needed to determine exactly when apoptosis and regression is occurring.

\section{Summary}

In conclusion, we provide evidence for the importance of TSP1/CD36 pathway in regulating basal skeletal muscle capillarity by showing that a chronic dosage of a TSP-1 mimetic for the CD36 pathway decreases skeletal muscle capillarity and VEGF expression. We did not find any differences in the VEGFR-2 expression or its phosphorylation status, nor was there greater skeletal muscle apoptosis. These data show that, despite the multifunctional effects of TSP-1 and its CD36 receptor, the primary consequence of elevating circulating TSP-1 (i.e. using a TSP-1 mimetic targeted to its CD36 receptor) relates to its anti-angiogenic function. These data may be useful in exploring therapeutic interventions for individuals with skeletal muscle dysfunction resulting from capillary regression.

\section{Acknowledgments}

The authors wish to thank Kathleen Roberts, W. Kyle Mandler, Sara Olenich, and Dharendrah Thapa for their assistance in the laboratory in preparing pumps and processing the tissues. We would also like to thank Dr. Josef Anrather of The Weill Cornell Medical College for his help and advice on CD36 analysis.

\section{Author Contributions}

Conceived and designed the experiments: IMO GNA. Performed the experiments: GNA DF JCS. Analyzed the data: GNA IMO. Contributed reagents/materials/analysis tools: IMO. Wrote the paper: GNA IMO. 


\section{References}

1. Breen EG, Johnson EC, Wagner H, Tseng HM, Sung LA, et al. (1996) Angiogenic growth factor mRNA responses in muscle to a single bout of exercise. Journal of Applied Physiology 81: 355-361.

2. Olfert IM, Howlett RA, Tang K, Dalton ND, Gu Y, et al. (2009) Muscle-specific VEGF deficiency greatly reduces exercise endurance in mice. The Journal of Physiology 587: 1755.

3. Roudier E, Gineste C, Wazna A, Dehghan K, Desplanches D, et al. (2010) Angio-adaptation in unloaded skeletal muscle: new insights into an early and muscle type-specific dynamic process. The Journal of Physiology 588: 45794591 .

4. Olfert IM, Birot O (2011) Importance of Anti-angiogenic Factors in the Regulation of Skeletal Muscle Angiogenesis. Microcirculation 18: 316-330.

5. Jack L (2000) The functions of thrombospondin-1 and-2. Current Opinion in Cell Biology 12: 634-640.

6. Jimenez B, Volpert OV, Crawford SE, Febbraio M, Silverstein RL, et al. (2000) Signals leading to apoptosis-dependent inhibition of neovascularization by thrombospondin-1. Nature medicine 6: 41-48.

7. Lange-Asschenfeldt B, Weninger W, Velasco P, Kyriakides TR, von Andrian $\mathrm{UH}$, et al. (2002) Increased and prolonged inflammation and angiogenesis in delayed-type hypersensitivity reactions elicited in the skin of thrombospondin-2deficient mice. Blood 99: 538-545.

8. Varma V, Yao-Borengasser A, Bodles AM, Rasouli N, Phanavanh B, et al. (2008) Thrombospondin-1 Is an Adipokine Associated With Obesity, Adipose Inflammation, and Insulin Resistance. Diabetes 57: 432-439.

9. Ridnour LA, Isenberg JS, Espey MG, Thomas DD, Roberts DD, et al. (2005) Nitric oxide regulates angiogenesis through a functional switch involving thrombospondin-1. Proceedings of the National Academy of Sciences of the United States of America 102: 13147-13152.

10. Kvansakul M, Adams JC, Hohenester E (2004) Structure of a thrombospondin C-terminal fragment reveals a novel calcium core in the type 3 repeats. EMBO J 23: $1223-1233$.

11. Chen H, Herndon ME, Lawler J (2000) The cell biology of thrombospondin-1. Matrix Biology 19: 597-614.

12. Febbraio M, Hajjar DP, Silverstein RL (2001) CD36: a class B scavenger receptor involved in angiogenesis, atherosclerosis, inflammation, and lipid metabolism. Journal of Clinical Investigation 108: 785-792.

13. Primo L, Ferrandi G, Roca C, Marchiò S, di Blasio L, et al. (2005) Identification of CD36 molecular features required for its in vitro angiostatic activity. The FASEB journal 19: 1713-1715.

14. Ren B, Yee KO, Lawler J, Khosravi-Far R (2006) Regulation of tumor angiogenesis by thrombospondin-1. Biochimica et Biophysica Acta (BBA) Reviews on Cancer 1765: 178-188.

15. Hoekstra R, de Vos FYFL, Eskens FALM, Gietema JA, van der Gaast A, et al. (2005) Phase I Safety, Pharmacokinetic, and Pharmacodynamic Study of the Thrombospondin-1-Mimetic Angiogenesis Inhibitor ABT-510 in Patients With Advanced Cancer. Journal of Clinical Oncology 23: 5188-5197.

16. Reiher FK, Volpert OV, Jimenez B, Crawford SE, Dinney CP, et al. (2002) Inhibition of tumor growth by systemic treatment with thrombospondin-1 peptide mimetics. International Journal of Cancer 98: 682-689.

17. Rusk A, McKeegan E, Haviv F, Majest S, Henkin J, et al. (2006) Preclinical Evaluation of Antiangiogenic Thrombospondin-1 Peptide Mimetics, ABT-526 and ABT-510, in Companion Dogs with Naturally Occurring Cancers. Clinical Cancer Research 12: 7444-7455.

18. Olfert IM, Breen EC, Gavin TP, Wagner PD (2006) Temporal thrombospondin-1 mRNA response in skeletal muscle exposed to acute and chronic exercise. Growth Factors 24: 253-259.

19. Olfert IM (2012) Unpublished Data

20. Frazier EP, Isenberg JS, Shiva S, Zhao L, Schlesinger P, et al. (2011) Agedependent regulation of skeletal muscle mitochondria by the thrombospondin-1 receptor CD47. Matrix Biology.

21. Dawson DW, Pearce SFA, Zhong R, Silverstein RL, Frazier WA, et al. (1997) CD36 mediates the in vitro inhibitory effects of thrombospondin-1 on endothelial cells. The Journal of cell biology 138: 707

22. Haviv F, Bradley MF, Kalvin DM, Schneider AJ, Davidson DJ, et al. (2005) Thrombospondin-1 mimetic peptide inhibitors of angiogenesis and tumor growth: design, synthesis, and optimization of pharmacokinetics and biological activities. Journal of medicinal chemistry 48: 2838-2846.

23. Anderson JC, Grammer JR, Wang W, Nabors LB, Henkin J, et al. (2007) ABT510, a modified type 1 repeat peptide of thrombospondin, inhibits malignant glioma growth in vivo by inhibiting angiogenesis. Cancer biology \& therapy 6 : 454 .

24. Greenaway J, Henkin J, Lawler J, Moorehead R, Petrik J (2009) ABT-510 induces tumor cell apoptosis and inhibits ovarian tumor growth in an orthotopic, syngeneic model of epithelial ovarian cancer. Molecular cancer therapeutics 8: 64.

25. Rusk A, McKeegan E, Haviv F, Majest S, Henkin J, et al. (2006) Preclinical evaluation of antiangiogenic thrombospondin- 1 peptide mimetics, ABT-526 and ABT-510, in companion dogs with naturally occurring cancers. Clinical cancer research 12: 7444 .

26. Yap R, Veliceasa D, Emmenegger U, Kerbel RS, McKay LM, et al. (2005) Metronomic low-dose chemotherapy boosts CD95-dependent antiangiogenic effect of the thrombospondin peptide ABT-510: a complementation antiangiogenic strategy. Clinical cancer research 11: 6678.

27. Campbell NE, Greenaway J, Henkin J, Moorehead RA, Petrik J The Thrombospondin-1 Mimetic ABT-510 Increases the Uptake and Effectiveness of Cisplatin and Paclitaxel in a Mouse Model of Epithelial Ovarian Cancer. Neoplasia Press Inc.

28. Isenberg JS, Yu C, Roberts DD (2008) Differential effects of ABT-510 and a CD36-binding peptide derived from the type 1 repeats of thrombospondin-1 on fatty acid uptake, nitric oxide signaling, and caspase activation in vascular cells. Biochemical Pharmacology 75: 875-882.

29. Hasina R, Martin LE, Kasza K, Jones CL, Jalil A, et al. (2009) ABT-510 is an effective chemopreventive agent in the mouse 4-nitroquinoline 1-oxide model of oral carcinogenesis. Cancer Prevention Research 2: 385.

30. Joyce D, Mulji G, Gutierrez L, Castellino F (2006) Evaluation of the Thrombospondin-1 analogue ABT-510 in the APCMin/+ mouse intestinal adenoma model. Journal of Clinical Oncology 24: 13545.

31. Mrázková O, Grim M, Carlson BM (1986) Enzymatic heterogeneity of the capillary bed of rat skeletal muscles. American Journal of Anatomy 177: 141148 .

32. Lojda Z (1979) Studies on dipeptidyl(amino)peptidase IV (glycyl-proline naphthylamidase). Histochemistry 59: 153-166.

33. Malek MH, Olfert IM (2009) Global deletion of thrombospondin 1 increases cardiac and skeletal muscle capillarity and exercise capacity in mice. Experimental physiology 94: 749-760.

34. Roudier E, Gineste C, Wazna A, Dehghan K, Desplanches D, et al. (2010) Angio-adaptation in unloaded skeletal muscle: new insights into an early and muscle type-specific dynamic process. The Journal of Physiology 588: 4579.

35. Bornstein P (2009) Thrombospondins function as regulators of angiogenesis. Journal of Cell Communication and Signaling 3: 189-200.

36. Lawler J (2000) The functions of thrombospondin-1 and-2. Current opinion in cell biology 12: 634-640.

37. Zhang X, Lawler J (2007) Thrombospondin-based antiangiogenic therapy. Microvascular research 74: 90-99.

38. Lawler J, Detmar M (2004) Tumor progression: the effects of thrombospondin-1 and -2. The International Journal of Biochemistry \& Cell Biology 36: 10381045 .

39. Kivela R, Silvennoinen M, Lehti M, Jalava S, Vihko V, et al. (2008) Exerciseinduced expression of angiogenic growth factors in skeletal muscle and in capillaries of healthy and diabetic mice. Cardiovascular Diabetology 7: 13.

40. Kivelä R, Silvennoinen M, Touvra A-M, Lehti TM, Kainulainen H, et al. (2006) Effects of experimental type 1 diabetes and exercise training on angiogenic gene expression and capillarization in skeletal muscle. The FASEB journal 20: 15701572 .

41. Duscha BD, Kraus WE, Keteyian SJ, Sullivan MJ, Green HJ, et al. (1999) Capillary density of skeletal muscle: A contributing mechanism for exercise intolerance in class II-III chronic heart failure independent of other peripheral alterations. Journal of the American College of Cardiology 33: 1956-1963.

42. Greenaway J, Lawler J, Moorehead R, Bornstein P, Lamarre J, et al. (2007) Thrombospondin-1 inhibits VEGF levels in the ovary directly by binding and internalization via the low density lipoprotein receptor-related protein-1 (LRP1). Journal of cellular physiology 210: 807-818.

43. Gupta K, Gupta P, Wild R, Ramakrishnan S, Hebbel R (1999) Binding and displacement of vascular endothelial growth factor (VEGF) by thrombospondin: Effect on human microvascular endothelial cell proliferation and angiogenesis. Angiogenesis 3: 147-158.

44. Iruela-Arispe ML, Lombardo M, Krutzsch HC, Lawler J, Roberts DD (1999) Inhibition of Angiogenesis by Thrombospondin-1 Is Mediated by 2 Independent Regions Within the Type 1 Repeats. Circulation 100: 1423-1431.

45. Rodríguez-Manzaneque JC, Lane TF, Ortega MA, Hynes RO, Lawler J, et al. (2001) Thrombospondin-1 suppresses spontaneous tumor growth and inhibits activation of matrix metalloproteinase- 9 and mobilization of vascular endothelial growth factor. Proceedings of the National Academy of Sciences of the United States of America 98: 12485.

46. Sun J, Hopkins BD, Tsujikawa K, Perruzzi C, Adini I, et al. (2009) Thrombospondin-1 modulates VEGF-A-mediated Akt signaling and capillary survival in the developing retina. American Journal of Physiology-Heart and Circulatory Physiology 296: H1344.

47. Simons M (2012) An Inside View: VEGF Receptor Trafficking and Signaling. Physiology 27: 213-222.

48. Kaur S, Martin-Manso G, Pendrak ML, Garfield SH, Isenberg JS, et al. (2010) Thrombospondin-1 Inhibits VEGF Receptor-2 Signaling by Disrupting Its Association with CD47. Journal of Biological Chemistry 285: 38923-38932.

49. Zhang X, Kazerounian S, Duquette M, Perruzzi C, Nagy JA, et al. (2009) Thrombospondin-1 modulates vascular endothelial growth factor activity at the receptor level. The FASEB journal 23: 3368-3376.

50. Zhang X, Kazerounian S, Duquette M, Perruzzi C, Nagy JA, et al. (2009) Thrombospondin-1 modulates vascular endothelial growth factor activity at the receptor level. The FASEB journal 23: 3368.

51. Reiher FK, Volpert OV, Jimenez B, Crawford SE, Dinney CP, et al. (2002) Inhibition of tumor growth by systemic treatment with thrombospondin 1 peptide mimetics. International journal of cancer 98: 682-689. 
52. Friedl P, Vischer P, Freyberg M (2002) The role of thrombospondin-1 in apoptosis. Cellular and molecular life sciences 59: 1347-1357.

53. Guo N, Krutzsch HC, Inman JK, Roberts DD (1997) Thrombospondin 1 and type I repeat peptides of thrombospondin 1 specifically induce apoptosis of endothelial cells. Cancer research 57: 1735.

54. Miao W-M, Lin Seng W, Duquette M, Lawler P, Laus C, et al. (2001) Thrombospondin-1 Type 1 Repeat Recombinant Proteins Inhibit Tumor Growth through Transforming Growth Factor- $\beta$-dependent and -independent Mechanisms. Cancer research 61: 7830-7839.

55. Nör J, Mitra RS, Sutorik MM, Mooney DJ, Castle VP, et al. (2000) Thrombospondin-1 induces endothelial cell apoptosis and inhibits angiogenesis by activating the caspase death pathway. Journal of vascular research 37: 209218.

56. Isenberg J, Frazier W, Roberts D (2008) Thrombospondins: from structure to therapeutics: Thrombospondin-1: a physiological regulator of nitric oxide signaling. Cellular and Molecular Life Sciences CMLS 65: 728-742.

57. Isenberg JS, Hyodo F, Matsumoto KI, Romeo MJ, Abu-Asab M, et al. (2007) Thrombospondin-1 limits ischemic tissue survival by inhibiting nitric oxidemediated vascular smooth muscle relaxation. Blood 109: 1945.

58. Isenberg JS, Ridnour LA, Perruccio EM, Espey MG, Wink DA, et al. (2005) Thrombospondin-1 inhibits endothelial cell responses to nitric oxide in a cGMPdependent manner. Proceedings of the National Academy of Sciences of the United States of America 102: 13141.

59. Wintergerst ES, Jelk J, Rahner C, Asmis R (2000) Apoptosis induced by oxidized low density lipoprotein in human monocyte-derived macrophages involves CD36 and activation of caspase-3. European Journal of Biochemistry 267: $6050-6059$
60. Meeson AP, Argilla M, Ko K, Witte L, Lang RA (1999) VEGF deprivationinduced apoptosis is a component of programmed capillary regression. Development 126: 1407-1415.

61. Tang K, Breen EC, Gerber H-P, Ferrara NMA, Wagner PD (2004) Capillary regression in vascular endothelial growth factor-deficient skeletal muscle. Physiological Genomics 18: 63-69.

62. Meeson A, Palmer M, Calfon M, Lang R (1996) A relationship between apoptosis and flow during programmed capillary regression is revealed by vital analysis. Development 122: 3929-3938.

63. Dimmeler S, Zeiher AM (2000) Endothelial Cell Apoptosis in Angiogenesis and Vessel Regression. Circulation research 87: 434-439.

64. Degens H, Alway SE (2006) Control of muscle size during disuse, disease, and aging. International journal of sports medicine 27: 94-99.

65. Siu PM, Pistilli EE, Butler DC, Alway SE (2005) Aging influences cellular and molecular responses of apoptosis to skeletal muscle unloading. American Journal of Physiology - Cell Physiology 288: C338-C349.

66. dalla Libera L, Zennaro R, Sandri M, Ambrosio GB, Vescovo G (1999) Apoptosis and atrophy in rat slow skeletal muscles in chronic heart failure. American Journal of Physiology - Cell Physiology 277: C982-C986.

67. Dupont-Versteegden EE (2005) Apoptosis in muscle atrophy: Relevance to sarcopenia. Experimental Gerontology 40: 473-481.

68. Allen DL, Linderman JK, Roy RR, Bigbee AJ, Grindeland RE, et al. (1997) Apoptosis: a mechanism contributing to remodeling of skeletal muscle in response to hindlimb unweighting. American Journal of Physiology - Cell Physiology 273: C579-C587. 


\title{
Expression of angiogenic regulators and skeletal muscle capillarity in selectively bred high aerobic capacity mice
}

\author{
Gerald N. Audet ${ }^{1}$, Thomas H. Meek ${ }^{2}$, Theodore Garland Jr ${ }^{2}$ and I. Mark Olfert ${ }^{1}$ \\ ${ }^{1}$ West Virginia University School of Medicine, Center for Cardiovascular and Respiratory Sciences, Division of Exercise Physiology, \\ Morgantown, WV 26508, USA \\ ${ }^{2}$ University of California-Riverside, Department of Biology, Riverside, CA 92521, USA
}

\begin{abstract}
Selective breeding for high voluntary wheel running in untrained mice has resulted in a 'mini muscle' (MM) phenotype, which has increased skeletal muscle capillarity compared with muscles from non-selected control lines. Vascular endothelial growth factor (VEGF) and thrombospondin-1 (TSP-1) are essential mediators of skeletal muscle angiogenesis; thus, we hypothesized that untrained MM mice with elevated muscle capillarity would have higher basal VEGF expression and lower basal TSP-1 expression, and potentially an exaggerated VEGF response to acute exercise. We examined skeletal muscle morphology and skeletal muscle protein expression of VEGF and TSP-1 in male mice from two (untrained) mouse lines selectively bred for high exercise capacity (MM and Non-MM), as well as one non-selected control mouse line (normal aerobic capacity). In the MM mice, gastrocnemius (GA) and plantaris (PLT) muscle capillarity (i.e. capillary-to-fibre ratio and capillary density) were greater compared with control mice $(P<0.05)$. In Non-MM mice, only muscle capillarity in PLT was greater than in control mice $(P<0.001)$. The soleus (SOL) showed no statistical differences in muscle capillarity among groups. In the GA, MM mice had 58\% greater basal VEGF $(P<0.05)$, with no statistical difference in basal TSP-1 when compared with control mice. In the PLT, MM mice had a 79\% increase in basal VEGF $(P<0.05)$ and a 39\% lower basal TSP-1 $(P<0.05)$ compared with the control animals. Non-MM mice showed no difference in basal VEGF in either the GA or the PLT compared with control mice. In contrast, basal TSP-1 was elevated in the PLT, but not in the GA, of Non-MM mice compared with control mice. Neither VEGF nor TSP-1 was significantly different in SOL muscle among the three mouse lines. In response to acute exercise, MM mice displayed a 41 and $28 \%$ increase $(P<0.05)$ in VEGF in the GA and PLT, respectively, whereas neither control nor Non-MM mice showed a significant VEGF response to acute exercise. In contrast, TSP-1 levels were decreased by $90 \%$ in GA $(P<0.05)$ but increased by $50 \%$ in PLT $(P<0.05)$ in response to acute exercise in MM mice. The SOL showed no response to exercise for either VEGF or TSP-1 for any of the mouse lines. These data, with the exception of the Non-MM plantaris muscle, suggest that elevated capillarity is associated with altered balance between positive and negative angiogenic regulators (i.e. VEGF versus TSP-1, respectively). Based on the greater capillarity and significant VEGF response to exercise in MM mice, these data suggest that VEGF expression may, at least in part, be genetically determined.
\end{abstract}

(Received 11 February 2011; accepted after revision 25 July 2011; first published online 31 July 2011)

Corresponding author I. M. Olfert: West Virginia University School of Medicine, Center for Cardiovascular and Respiratory Sciences, Division of Exercise Physiology, PO Box 9105, Morgantown, WV 26508, USA.

Email: molfert@hsc.wvu.edu 
The importance of genetic influences in determining aerobic exercise capacity has been highlighted by selective breeding approaches used to developed high aerobic capacity lines of rodents (Swallow et al. 2009; Feder et al. 2010). For example, Koch \& Britton (2001) have developed separate high (HCR) and low aerobic capacity rat (LCR) lines via respective breeding of rats for either high or low endurance capacity on a motorized treadmill. This has resulted in a diverging phenotype, where HCRs have a higher cardiovascular (Koch et al. 1999; Howlett et al. 2009) and respiratory capacity (Henderson et al. 2002) and increased skeletal muscle metabolism (Howlett et al. 2003), underpinning their greater innate aerobic endurance capacity compared with LCRs. Data obtained from this artificial selection experiment also highlights the importance of reduced/impaired physical activity for the pathogenesis of chronic disease, as the incidence of some cardiovascular risk factors is significantly greater in LCRs compared with HCRs (Wisloff et al. 2005).

Continuous selective breeding for high voluntary wheel running in laboratory house mice has also resulted in the development of four replicate lines of mice with a significantly increased aerobic exercise capacity (Swallow et al. 1998a,b; Garland et al. 2011). The 'gene pool' of the original outbred population of mice from which this experiment originated included a Mendelian recessive allele whose most notable feature is to cause a $50 \%$ reduction in hindlimb muscle mass when present in the homozygous condition (Garland et al. 2002; Houle-Leroy et al.2003). This 'mini-muscle' phenotype was favoured by the selective breeding regimen and increased in frequency in two of the four replicate selected lines (Garland et al. 2002). Further study of the mini-muscle mice (MM mice) has indicated several distinct physiological characteristics that may be advantageous with respect to wheel running. The most distinct feature is a significant reduction in the mass of most hindlimb muscles, but also includes fibre-type alterations favouring more oxidative fibres, increased tibia length (Syme et al. 2005; Guderley et al. 2008; McGillivray et al. 2009; Wong et al. 2009), increased mitochondrial enzymatic activity (Guderley et al. 2006), a smaller overall body mass (Swallow et al. $1999)$ and an increased maximal oxygen uptake $\left(\dot{V}_{\mathrm{O}_{2} \max }\right)$ when tested in hypoxia (Rezende et al. 2006a,b). More recently, it has also been shown that MM mice exhibit greater capillarity in the gastrocnemius compared with mice that have normal-sized muscles, especially around the medium and large fibres $\left(>2000 \mu \mathrm{m}^{2}\right.$; Wong et al. 2009).

Given that oxygen delivery to skeletal muscle is dependent on gas exchange across the microvascular capillary bed, one important mechanism to increase oxygen delivery to working muscle is to increase the number and density of capillaries surrounding the fibres (Andersen \& Henriksson, 1977; Wagner, 2003). Two potentially important mediators in the angiogenic process are vascular endothelial growth factor (VEGF; a positive angiogenic factor) and thrombospondin-1 (TSP-1; a negative angiogenic factor). In normal skeletal muscle, it has been shown that acute aerobic exercise increases VEGF (Breen et al. 1996; Gavin \& Wagner, 2001; Olfert et al. 2001; Gustafsson et al. 2002; Lloyd et al. 2003) and TSP-1 gene expression (Olfert et al. 2006), and that chronic or repeated exercise bouts ultimately decrease TSP-1 mRNA while VEGF mRNA remains elevated (Gavin \& Wagner, 2001; Olfert et al. 2001; Waters et al. 2004). Indeed, the importance of VEGF in regulating skeletal muscle capillarity is emphasized by recent evidence demonstrating that significant losses in VEGF result in reduced skeletal muscle capillarity (Tang et al. 2004; Olfert et al. 2009) and altered vascular function (Lee et al. 2007). Moreover, training-induced skeletal muscle angiogenesis is absent in VEGF-deficient mice (Olfert et al. 2010). By the same token, the importance of TSP-1 expression in preventing hypercapillarization of the skeletal muscle vascular bed has been shown using TSP-1 knockout mice (Malek \& Olfert, 2009).

Given the importance of VEGF and TSP-1 to skeletal muscle structure and function, we sought to examine VEGF and TSP-1 expression from two of the four selected mouse lines with inherited high aerobic capacity [one with the MM phenotype and another without the MM phenotype (i.e. Non-MM)] compared with a non-selected control line. We chose to examine two high capacity lines in our study, MM (Garland designated line 3) and Non-MM (Garland designated line 8), in order to more generally evaluate the high aerobic capacity phenotype, and not only the MM phenotype, compared with a non-selected control line (Garland designated line 2). In essence, the Non-MM mouse serves as an additional high capacity experimental group compared with the control line, but also as an additional high capacity group compared with MM mice (which have a very distinctive mini-muscle phenotype). For each of the three mouse lines, we examined basal expression and the response to acute exercise of VEGF and TSP-1 protein in the triceps surae hindlimb muscle. As both the MM and the NonMM mice have increased maximal oxygen consumption and treadmill endurance capacity during forced exercise compared with mice from control lines (Meek et al. 2009), and it has been shown that MM mice display elevated gastrocnemius muscle capillarity (Wong et al. 2009), it was first hypothesized that Non-MM mice would also have elevated skeletal muscle capillarity compared with control mice and second, that basal levels of VEGF would be higher in both MM and Non-MM high aerobic capacity mice compared with control mice. Moreover, given that all three mouse lines were untrained, we reasoned that we might see an exaggerated gene response to acute exercise in the high aerobic capacity lines compared with 
control animals due to the selection of genetic influences (resulting from the selected breeding strategy) that would favour greater skeletal muscle capillarity and high aerobic capacity mice, in the absence of training. On the other hand, as the high aerobic capacity lines are expected to have elevated muscle capillarity (and in normal mice elevated skeletal muscle capillarity has been associated with reduced VEGF responsiveness to exercise, at least in the context of training), it could also be hypothesized that simply having elevated capillarity (even in the absence of training) might diminish or attenuate the VEGF response to exercise in the high capacity selected lines compared with control mice.

In this study, we report data from the triceps surae muscles (which are known to have varying oxidative and glycolytic capacity) in order to identify potential differences in the skeletal muscle angiogenic response between muscles; but more specifically, we sought to exploit the innate difference between the respective mouse lines as an approach that might be a useful in unravelling the environmental versus genetic influences on skeletal muscle vascularization.

\section{Methods}

\section{Animals}

Male MM mice (laboratory designation selected line 3, $n=12$ ), Non-MM mice (selected line $8, n=12$ ) and control mice (line 2, $n=12$ ) were bred and raised at the University of California, Riverside. For a comprehensive review on the breeding and selection process, please see Swallow et al. (1998a). Briefly, eight independent lines of mice were created in the early 1990s from a common population of outbred Hsd:ICR laboratory mice. All lines were provided with running wheels for 6 days when the mice were $\sim 6-8$ weeks of age. In the four selected lines, the top-running male and female from within each of $\sim 10$ families, each generation, were used for breeding and the continuation of the line. The breeders were selected for the greatest distance run on the fifth and sixth day of wheel exposure. The other four lines were bred at random with respect to the amount of running, and served as control lines. After $\sim 16$ generations, the four 'high runner' lines ran approximately 2.7 times as far as the control lines, and had begun to display a number of characteristics unique to these lines (Swallow et al. 1998b, 2001, 2009; Koteja et al. 1999). In the present study, the mouse lines we examined came from the 54th generation, at which time the four selected lines were running more than three times further than control lines on a daily basis (T. Garland, unpublished observations). All procedures that involved animals were approved by the University of California, Riverside, Institutional Animal Care and Use Committee.

\section{Acute exercise protocol}

A subgroup of mice $(n=6)$ in each line were randomly assigned to perform a single bout of acute exercise in order to access growth factor response to an angiogenic stimulus. These mice ran in a custom-built treadmill (Meek et al. 2009). The clear Plexiglass lane in which the mouse ran had dimensions $6.5 \mathrm{~cm}$ wide, $12.5 \mathrm{~cm}$ high and $44 \mathrm{~cm}$ long. All animals were familiarized with the treadmill for $5 \mathrm{~min}$ prior to the acute exercise bouts. After this initial period, mice ran for $1 \mathrm{~h}$ at $20 \mathrm{~m} \mathrm{~min}^{-1}$ with $10 \mathrm{deg}$ incline. A run time of $1 \mathrm{~h}$ was selected because it has been shown to elicit a robust exercise-induced angiogenic gene response in rodents (Breen et al. 1996; Olfert et al. 2001). Animals were killed $4 \mathrm{~h}$ after the acute exercise at a time point known to correspond to peak VEGF protein expression (I. M. Olfert, unpublished data).

\section{Tissue preparations}

Each animal was killed by $\mathrm{CO}_{2}$ inhalation, and the triceps surae [gastrocnemius (GA), soleus (SOL) and plantaris (PLT)] muscles from each leg were immediately excised. Muscles from the right leg were weighed and immediately flash frozen in liquid $\mathrm{N}_{2}$, then later processed for VEGF protein expression. Muscles from the left leg were mounted on cork disks and quickly frozen in isopentane cooled in liquid $\mathrm{N}_{2}$, then processed later for morphometrical analysis. All samples were then stored at $-80^{\circ} \mathrm{C}$ until processed for molecular or morphological analyses.

\section{Morphometry}

Frozen tissue was cut using a $-20^{\circ} \mathrm{C}$ cyromicrotome (Jung-Reichert Cryocut 1800; Cambridge Instruments, Nussloch, Germany) to yield $10-\mu \mathrm{m}$-thick transverse sections. Great care was taken to ensure that the cryosectioned muscles were cut along the transverse plane. All tissues were stained for capillarity using the lead nitrate-ATPase method described by Rosenblatt et al. (1987). A light microscope (Nikon Eclipse 80i; Nikon Instruments Inc.) was used to digitally acquire (Photometrics CoolSnap ES; Photometrics, Tucson, AZ, USA) $\times 20$ images of sectioned muscles in a checkerboard fashion across the entire muscle. Capillary and myofibre counting was performed by a single individual, who was blinded to the identity of each of the samples. For the gastrocnemius muscle, we obtained images in a checkerboard fashion across the entire muscle, thus both superficial and deep regions within the gastrocnemius were included in the analysis. For the plantaris and soleus muscles, the entire muscle was imaged and analysed. Counting was performed by visualization from acquired images using a custom program in MATLAB 
Table 1. Body, heart and hindlimb muscle mass (means \pm SEM)

\begin{tabular}{|c|c|c|c|c|}
\hline & Control & $\mathrm{MM}$ & Non-MM & $\begin{array}{c}P \text { value for } \\
\text { three-group comparison }\end{array}$ \\
\hline Body mass (g) & $32.8 \pm 0.56$ & $29.9 \pm 0.83^{*} \dagger$ & $32.5 \pm 0.55$ & 0.01 \\
\hline Heart mass (mg) & $152.4 \pm 3.18$ & $161.2 \pm 4.81$ & $154.5 \pm 3.04$ & 0.24 \\
\hline Heart mass/body mass $\left(\mathrm{mg} \mathrm{g}^{-1}\right)$ & $4.65 \pm 0.11$ & $5.39 \pm 0.08^{*} \dagger$ & $4.76 \pm 0.12$ & $<0.01$ \\
\hline Gastrocnemius mass (mg) & $123.9 \pm 4.23$ & $59.2 \pm 2.08^{*} \dagger$ & $134.3 \pm 3.22$ & $<0.01$ \\
\hline Gastrocnemius mass/body mass $\left(\mathrm{mg} \mathrm{g}^{-1}\right)$ & $3.78 \pm 0.08$ & $1.98 \pm 0.05^{*} \dagger$ & $4.13 \pm 0.08^{*}$ & $<0.01$ \\
\hline Plantaris mass (mg) & $14.2 \pm 0.53$ & $9.8 \pm 0.62 * \dagger$ & $12.9 \pm 0.35$ & 0.01 \\
\hline Plantaris mass/body mass ( $\mathrm{mg} \mathrm{g}^{-1}$ ) & $0.43 \pm 0.03$ & $0.33 \pm 0.03^{*}$ & $0.40 \pm 0.03$ & 0.049 \\
\hline Soleus mass (mg) & $7.6 \pm 0.93$ & $9.8 \pm 0.93 * \dagger$ & $6.4 \pm 1.04$ & $<0.01$ \\
\hline Soleus mass/body mass ( $\mathrm{mg} \mathrm{g}^{-1}$ ) & $0.23 \pm 0.02$ & $0.51 \pm 0.02 * \dagger$ & $0.20 \pm 0.01$ & $<0.01$ \\
\hline
\end{tabular}

(version 7.0.0.27; The Mathworks, Natick, MA, USA), allowing the operator to visually mark and count the capillaries and fibres on each image. Capillary-to-fibre ratio (C:F; number of capillaries/number muscle fibres), capillary density (CD; number of capillaries/muscle fibre area) and fibre cross-sectional area (FCSA) were separately calculated for the gastrocnemius, soleus and plantaris from a minimum of 175 images per mouse line.

\section{Protein analysis}

The GA, SOL and PLT muscles from each group were separately homogenized in a lysis buffer containing $50 \mathrm{mM}$ Tris-HCl (pH 7.4), $150 \mathrm{~mm} \mathrm{NaCl,} \mathrm{0.5 \%} \mathrm{Triton} \mathrm{X-100} \mathrm{and}$ protease inhibitors (Complete ${ }^{\mathrm{TM}}$ Tablet; Roche Applied Science, Indianapolis, IN, USA). Homogenates were centrifuged at $4^{\circ} \mathrm{C}, 8000 \mathrm{~g}$ for $10 \mathrm{~min}$, and supernatants removed and placed in new tubes. Total protein was measured by the bicinchoninic acid method (BCA protein assay kit; Bio-Rad laboratories, Hercules, CA, USA).

Quantification of VEGF was made from a total of $100 \mu \mathrm{g}$ of protein using a commercially available ELISA kit according to the manufacturer's instructions (Model MMV00; R\&D Systems, Minneapolis, MN, USA).

Denatured samples $(20 \mu \mathrm{g})$ from GA and PLT muscles were separated on a 3-8\% SDS-PAGE and blotted onto a $0.45 \mu \mathrm{m}$ nitrocellulose membrane (Pierce nitrocellulose membrane; Thermo Scientific, Rockford, IL, USA). After blocking with $5 \%$ fat-free milk, membranes were probed using antibodies against TSP-1 (1:250 dilution; clone A6.1, no. 399300; Invitrogen, Burlington, ON, Canada), $\beta$-tubulin (1:1000 dilution; no. 2148; Cell Signaling, Danvers, MA, USA), horseradish peroxidaseconjugated anti-mouse (1:1000 dilution; no.p0260; Dako, GE Healthcare, Piscataway, NJ, USA) and horseradish peroxidase-conjugated anti-rabbit (1:1000 dilution; no.p0217; Dako). Proteins were visualized using chemiluminescence detection (Pierce ECL; Thermo Scientific) and digitally imaged (G:BOX Gel imager; Syngene, Cambridge, UK). Images were captured using Genesnap software (version 7.01; Syngene). Equal protein loading was verified by immunodetection of $\beta$-tubulin as our loading control. Quantification of protein expression levels were carried out using NIH ImageJ Software (version 1.62) and expressed as densitometric arbitrary units (a.u.). The TSP-1 expression was normalized to $\beta$-tubulin expression level. Owing to insufficient sample volume, we were not able to run Western analysis of TSP-1 on the SOL muscle.

\section{Statistics}

All data are presented as means \pm SEM. To examine body mass, organ masses and muscle capillarity (measured only in basal conditions), we used a one-way ANOVA comparing the three lines of mice. To examine protein expression, we used a two-way ANOVA comparing the three lines of mice in basal conditions and in response to acute exercise, while also testing for an interaction between line and exercise condition. Separate analyses were performed for each of the three muscles studied (StatView, 5.0.1). When a main effect was observed, post hoc testing was performed using Student's unpaired $t$ test. An $\alpha$ level of $P<0.05$ was selected for statistical significance.

\section{Results}

\section{Body and muscle masses}

Body mass differed significantly among lines $(P=0.006)$. As seen in Table 1, MM mice had 9\% lower body mass than Non-MM mice $(P=0.008)$ and $10 \%$ lower than control mice $(P=0.003)$. The MM mice also had a $16 \%$ larger heart mass (per gram body mass) compared with control line mice $(P<0.001)$ and a $13 \%$ increase over the NonMM line $(P<0.001)$. There was no statistical difference in relative heart mass between the Non-MM and the control animals.

All three muscles of the triceps surae differed significantly in mass among all lines (GA, $P<0.001$; SOL, $P<0.01$; and PLT, $P<0.05$ ), even when expressed relative to body mass (GA, $P<0.001$; SOL, $P<0.001$; and PLT, $P<0.05$; Table 1 ). The MM mice had a $56 \%$ decrease in 
GA mass compared with Non-MM line $(P<0.001)$ and a $52 \%$ reduction compared with control mice $(P<0.001)$. The Non-MM line also had $8 \%$ greater GA mass than control mice $(P=0.036)$. On a mass-specific basis, the MM mice had a $48 \%$ reduction compared with the control mice $(P<0.001)$ and a 52\% reduction compared with the Non-MM line $(P<0.001)$. The Non-MM line also had an $8 \%$ increase when corrected for body mass compared with the control mice $(P<0.001)$. As expected (Guderley et al. 2008; Wong et al. 2009), superficial portions of the GA muscle in MM mice showed the distinct appearance of many small fibres not present in either of the other lines (Fig. 1).

The PLT of the MM mouse was also 32\% smaller than in the Non-MM line $(P=0.039)$ and $45 \%$ smaller than in control mice $(P=0.004)$. There was no difference between the Non-MM line and control mice. On a mass-specific basis, only the MM mice and control mice showed any statistical difference, with a $30 \%$ reduction in the MM PLT mass $(P=0.015)$ compared with the control animals.

The SOL of MM mice was 53\% larger than in the NonMM mice $(P<0.001)$ and $29 \%$ larger than in the control mice $(P=0.005)$. There was no statistical difference between the Non-MM line and the control mice. On a mass-specific basis, the MM line had $155 \%$ greater SOL mass than the Non-MM line $(P<0.001)$ and a $122 \%$ greater mass than the control line $(P<0.001)$. There was no difference between the Non-MM line and the control animals after correcting for body mass.

\section{Morphometry}

There was a significant main effect for C:F, CD and FCSA among all groups for the GA (all $P<0.001$ ) and PLT (all $P<0.05$ ), but not for the SOL muscle. In the GA, MM mice had a $25 \%$ greater C:F and $43 \%$ greater CD than the Non-MM line $(P<0.001)$ and $38 \%$ greater $\mathrm{C}: \mathrm{F}$ and $46 \%$ greater $\mathrm{CD}$ than the control mice $(P<0.001$; Fig. 2$)$. There was no difference in C:F or CD between the Non-MM and control mice for the GA. The GA FCSA was decreased by 39 and $36 \%$ in the MM mice compared to Non-MM and control mice, respectively $(P<0.001$; Fig. 2$)$.

In the PLT, there was no difference in C:F, CD and FCSA between MM mice and the Non-MM line (Fig. 2); however, the MM mice and Non-MM mice had a 35 $(P=0.002)$ and $27 \%(P=0.005)$ greater $\mathrm{C}: \mathrm{F}$, and a 24 $(P<0.034)$ and $31 \%(P<0.003)$ greater CD in the PLT compared with control mice (Fig. 2).

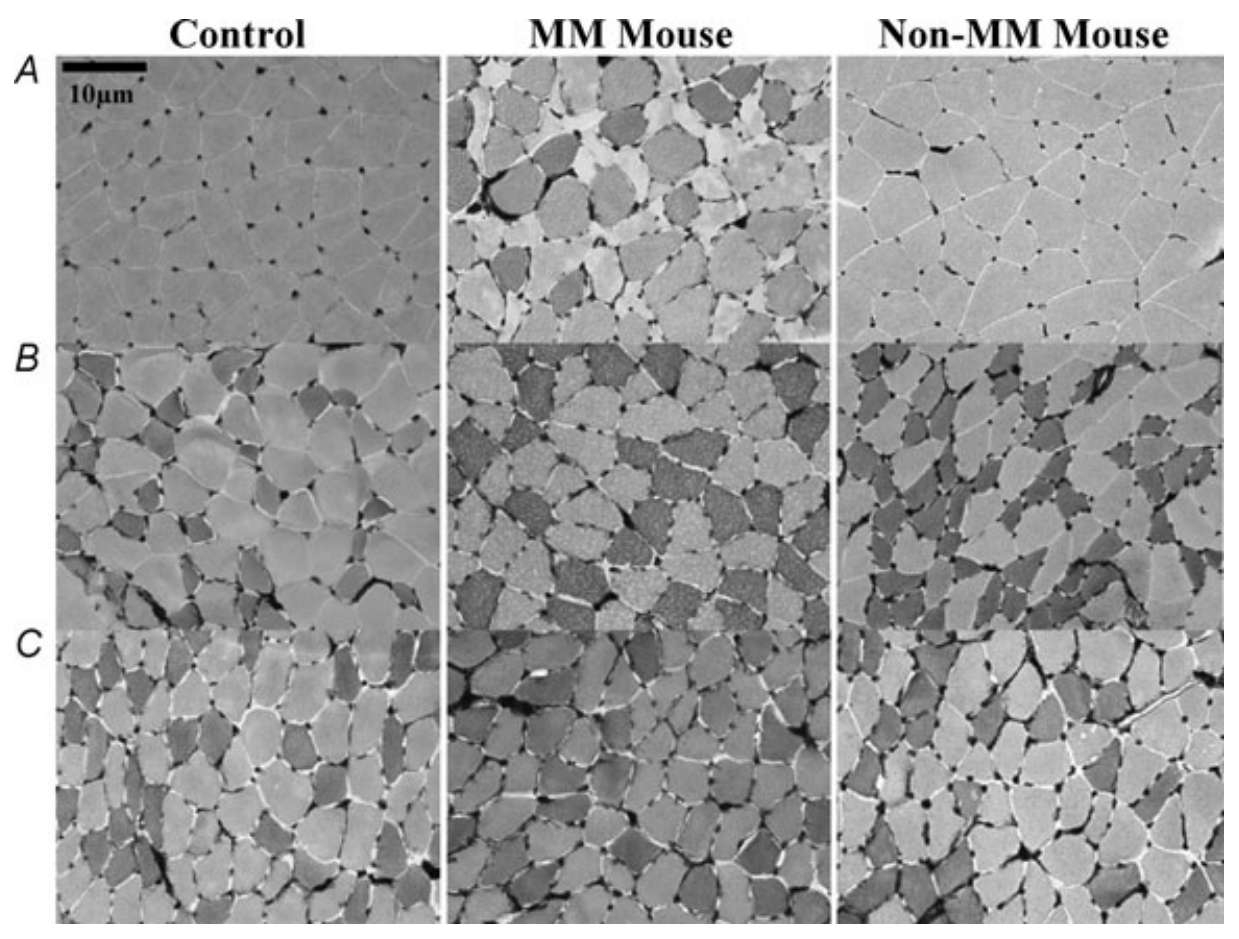

Figure 1. Representative images of triceps surea muscles

$A$, superficial gastrocnemius. $B$, soleus. $C$, plantaris. Capillaries were stained using lead-ATPase appear as black dots outside the muscle. The presence of very small muscle fibres in the gastrocnemius muscle $(A)$ is evident in MM mice compared with control and Non-MM mice. Capillary-to-fibre ratio is significantly elevated in gastrocnemius muscle of MM mice $(A)$ compared with control and Non-MM mice $(P<0.001)$, and significantly greater in plantaris muscle of MM mice $(B)$ compared with control mice $(P<0.001)$, but not Non-MM mice. No difference in soleus muscle capillarity was observed between the groups $(C)$. 
Table 2. Muscle fibre-type composition

\begin{tabular}{|c|c|c|c|c|c|c|c|c|}
\hline & \multicolumn{4}{|c|}{ Type I (\%) } & \multicolumn{4}{|c|}{ Type II (\%) } \\
\hline & Control & MM & Non-MM & $P$ value & Control & MM & Non-MM & $P$ value \\
\hline Gastrocnemius (deep) & $20 \pm 4$ & $30 \pm 4 \dagger$ & $24 \pm 3$ & 0.18 & $79 \pm 4$ & $70 \pm 4 \dagger$ & $75 \pm 3$ & 0.15 \\
\hline Gastrocnemius (superficial) & $2 \pm 1$ & $12 \pm 3^{*}$ & $7 \pm 3$ & 0.04 & $98 \pm 1$ & $89 \pm 3^{*}$ & $95 \pm 2$ & 0.02 \\
\hline Plantaris & $38 \pm 6$ & $38 \pm 1$ & $39 \pm 2$ & 0.96 & $62 \pm 6$ & $62 \pm 2$ & $61 \pm 2$ & 0.91 \\
\hline Soleus & $40 \pm 2$ & $32 \pm 5$ & $39 \pm 4$ & 0.32 & $61 \pm 3$ & $68 \pm 5$ & $61 \pm 3$ & 0.29 \\
\hline
\end{tabular}

$P$ value represents ANOVA comparison among three groups; ${ }^{*} P<0.05$ compared with control line; and $\dagger P=0.07$ compared with control line.

There was not a significant main effect for C:F, CD or FCSA in the SOL (data not shown).

There was a significant main effect for fibre type for the superficial GA between the mouse lines $(P<0.05)$. Post hoc analysis revealed that MM mice had $86 \%$ more type I fibres $(P=0.014)$ and $10 \%$ fewer type II fibres $(P=0.007)$ than control mice (Table 2). In the deep GA, a significant main effect for fibre type was not found between the mouse lines (type I, $P=0.179$; and type II, $P=0.154$ ); however, this region of the GA exhibited a similar trend towards having an increase in the proportion of type I and decrease in proportion of type II myofibres in MM compared with control mice. There was no significant difference between MM mice and Non-MM mice in either portion of the GA. No significant differences in fibre type between the mouse lines in either the PLT or SOL were observed.

\section{Protein expression of VEGF}

Basal levels. There was a main effect among mouse lines for basal VEGF expression in the GA $(P=0.017)$ and the PLT muscles $(P=0.031)$. In the GA, MM mice had a $58 \%$ higher basal expression of VEGF compared with control mice ( $P=0.006$; Fig. 3$)$. Although basal VEGF expression was also $33 \%$ greater in Non-MM compared with control mice, this difference did not reach statistical significance $(P=0.055)$. There was no significant difference in basal VEGF expression between MM and Non-MM mice.

In the PLT muscle, we observed $79 \%$ greater VEGF expression in MM mice compared with control mice $(P=0.010)$, but no significant difference between MM and Non-MM mice $(P=0.081)$. There was also no significant difference between Non-MM mice and control mice, despite a similar tendency for $28 \%$ greater VEGF levels in Non-MM mice $(P=0.251)$, like that seen in the gastrocnemius.

No significant main effect for basal VEGF expression was observed in the SOL muscle (data not shown).

Response to acute exercise. There was a main effect for VEGF response among mouse lines $(P=0.0003)$ and a significant exercise $\times$ line interaction in the GA $(P=0.047$; Fig. 3$)$. Only the MM mice showed a significant VEGF response to acute exercise, resulting in
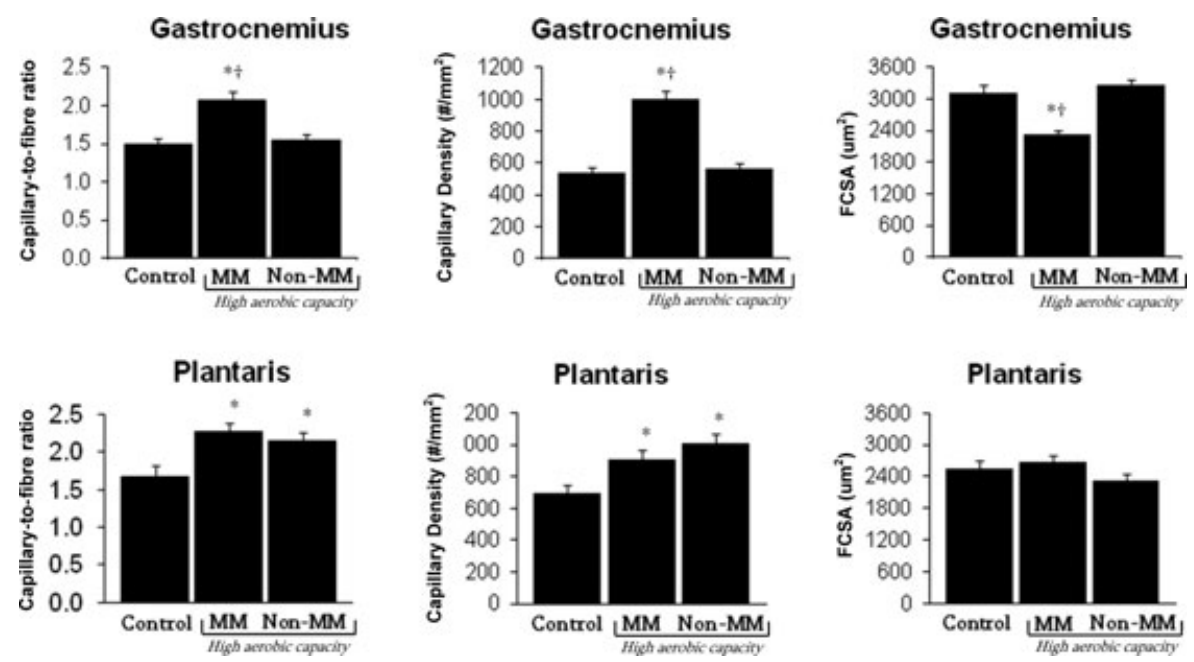

Figure 2. Capillary-to-fibre ratio (C:F), capillary density (CD) and fibre cross-sectional area (FSCA) of the triceps surae muscles

In the gastrocnemius, MM mice had an increase in C:F and CD and a decrease in FCSA compared with both control and Non-MM mice. In plantaris, MM and Non-MM had an increased C:F and CD compared with control mice. ${ }^{*} P<0.05$ compared with control mice; and $\uparrow P<0.05$ compared with Non-MM mice. 
a $41 \%$ increase in VEGF in the GA from basal levels $(P=0.009)$. The control mice had a very similar $38 \%$ increase in VEGF from baseline, but this did not reach statistical significance $(P=0.081)$, which was probably due to the greater variability observed in the VEGF response to exercise in these mice. Surprisingly, there was no VEGF response to exercise in the Non-MM line GA.

There was no statistically significant interaction between the VEGF response to exercise and line in either of the other muscles studied (i.e. PLT and SOL). However, similar to the GA, VEGF in the PLT increased $28 \%$ in response to exercise in MM mice $(P=0.038)$. Non-MM mice showed a non-significant increase $(21 \% ; P=0.158)$ for VEGF in the plantaris, while control mice did not respond to exercise.

No significant difference between groups for the VEGF response to exercise was observed in the SOL muscle (data not shown).
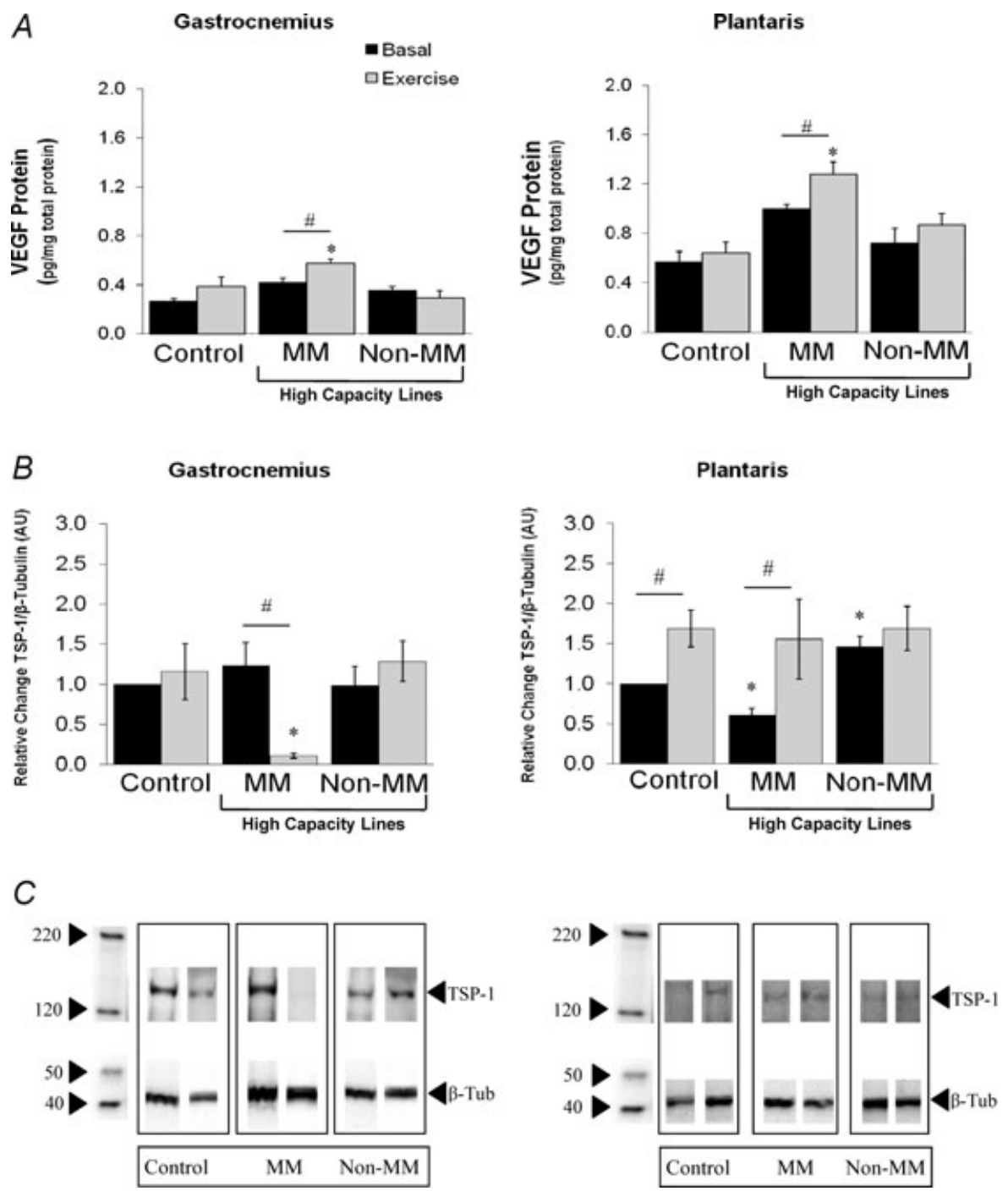

Figure 3. Comparison of basal and postexercise levels of vascular endothelial growth factor (VEGF) and thrombospondin-1 (TSP-1) in the triceps surae muscles

$A$, there was a statistically significant interaction between acute exercise and VEGF levels in the gastrocnemius (GA) and plantaris (PLT). In both muscles, MM mice had increased basal levels of VEGF compared with control animals, and a significant VEGF response to acute exercise. The VEGF levels did not differ between the Non-MM and control mice in either muscle, although there was a trend for an increase in the GA $(P=0.055)$. $B$, there was a statistically significant interaction between acute exercise and TSP-1 levels in the GA and PLT. In basal conditions in the PLT, the MM mice had lower TSP-1 levels than either the control or Non-MM mice, and in the GA and PLT the MM mice had a significant TSP-1 response to acute exercise. The Non-MM mice had significantly higher basal levels of TSP-1 in the PLT than control mice. Also in the PLT, control animals had a significant TSP-1 response to acute exercise. $C$, representative Western blots from TSP-1 analysis $(B)$. ${ }^{*} P<0.05$ compared with control mice, same exercise state (basal or acute exercise); and \#P<0.05 compared with basal levels. 


\section{Protein expression of TSP-1}

Basal levels. Basal levels of TSP-1 in the GA muscle were not different among mouse lines. However, a significant main effect for basal TSP-1 expression in the PLT was observed among mouse lines. In the PLT, MM mice had 39\% lower TSP-1 levels compared with control mice $(P<0.01)$ and $58 \%$ lower compared with the Non-MM mice $(P<0.01$; Fig. 3$)$. Non-MM mice exhibited a $46 \%$ increase in basal TSP-1 compared with control mice $(P<0.001$; Fig. 3$)$.

Response to acute exercise. There was a significant exercise $\times$ line interaction in the GA $(P=0.014$; Fig. 3$)$. In the GA, control mice and Non-MM mice showed no statistical TSP-1 response to acute exercise, whereas MM mice exhibited a 90\% decrease in TSP-1 $(P=0.003)$. In the PLT, there was also a significant effect for TSP-1 in response to acute exercise $(P=0.005)$, where TSP-1 expression increased by $47 \%(P<0.01)$ in control mice and by $55 \%(P<0.05)$ in MM mice (Fig. 3$)$. There was no significant acute exercise response in Non-MM mice (Fig. 3).

\section{Discussion}

The main finding of this study is that elevated skeletal muscle capillarity in MM mice bred for high aerobic capacity is associated with an altered balance between VEGF and TSP-1 protein expression (Fig. 3). These data are consistent with the observations that VEGF and TSP-1 are essential regulators of the skeletal muscle microvascular bed. Surprisingly, in Non-MM mice a similar diverging correlation between VEGF and TSP1 in relation to changes in muscle capillarity was not found in the PLT muscle. In general, however, these data support the notion that VEGF and TSP-1 are key physiological regulators of angiogenesis within skeletal muscle. Moreover, when comparing the basal expression of these factors in MM mice against control mice, these are among the first data to suggest that muscle capillarity and the capacity for exercise-induced angio-adaptation may, in part, be accounted for by inherited traits related to the expression of VEGF, and possibly TSP-1, within skeletal muscle.

\section{Basal expression of VEGF and TSP-1 correlates with elevated skeletal muscle capillarity in MM mice, but not in Non-MM mice}

In this study, we report that MM mice have increased capillarity in the both the GA and the PLT (Fig. 2), which is associated with elevated basal expression of VEGF in each muscle (Fig. 3). At the same time, we observed no difference in basal skeletal muscle TSP-1 expression in the GA, but a significant decrease in the PLT (Fig. 3) which, when combined with the elevated expression of VEGF in both GA and PLT, is consistent with a proangiogenic environment within the muscle. In contrast, neither capillarity nor VEGF expression was different in the SOL muscle of MM or Non-MM mice compared with control mice.

In Non-MM mice, which are also high aerobic capacity mice but without the unique 'mini-muscle' phenotype, we found that only the PLT had elevated capillarity, whereas neither GA nor SOL muscle capillarity was different compared with control animals (Fig. 2). Although there was a trend for elevated basal VEGF expression in the PLT of Non-MM mice $(P=0.07)$, we also found elevated basal TSP-1 expression $(P<0.01)$, which together could be argued as an anti-angiogenic state rather than pro-angiogenic. Nonetheless, an increase in PLT muscle capillarity was observed in Non-MM mice (Fig. 2). In contrast, no significant changes in basal VEGF were seen in either the GA or SOL muscles of Non-MM mice. Likewise, TSP-1 was not different in GA muscle, and no differences in capillarity were seen in either the GA or the SOL of Non-MM mice compared with control animals.

Taken together, the elevated muscle capillarity in the GA of MM mice is most probably explained by elevated basal VEGF. In the PLT, elevated muscle capillarity occurred in the presence of decreased basal TSP-1 expression and little or no increase in VEGF, suggesting that TSP-1 may also be playing an important role, at least in the PLT of MM mice. Despite the differences in these individual muscles, there is growing evidence that physiological regulation of angiogenesis is dependent on the balance between positive and negative angiogenic proteins such that the ratio of VEGF to TSP-1 expression may actually be more important than their individual expression levels per se (Olfert \& Birot, 2011). The present results support the notion of an altered balance leading to capillary adaptation, with the notable exception of the PLT muscle in Non-MM mice. One explanation for this discrepancy may be that other angiogenic regulators (such as endostatin, angiopoetins and their receptors, matrix metalloproteinases) are also playing a role, but are not accounted for in this study. Indeed, while we focused on VEGF and TSP-1 because of their recognized prominence in effecting changes in the skeletal muscle vascular bed, it is entirely possible (and likely) that other regulators also contribute to the basal angiogenic state (Olfert \& Birot, 2011).

It is interesting to note that increased capillarity occurred predominately in skeletal muscle composed of glycolytic myofibres (i.e. GA and PLT) and not in muscle composed of mostly oxidative myofibres (i.e. SOL). This observation is consistent in both high aerobic selected lines (i.e. MM and Non-MM), and might suggest that muscles 
with greater glycolytic metabolic profiles experienced the greatest amount of evolutionary change in these selectively bred lines of mice. This idea is consistent with the observation that glycolytic myofibres may be principally responsible for driving the angiogenic response to acute exercise (Birot et al. 2003; Waters et al. 2004). It may also be that muscles having a predominant role in locomotor activity (i.e. GA and PLT) would be the most evolved in these mice, whereas postural skeletal muscles (such as the SOL) are less likely to have been influenced.

\section{Exercise capacity, angiogenesis and VEGF}

It remains clear that muscles found to have elevated basal VEGF expression are also the muscles with elevated capillarity (Fig. 4). In TSP-1 knockout mice, increases in skeletal muscle capillarity (achieved without exercise training) are also associated with greater exercise capacity (Malek \& Olfert, 2009), whereas losses in muscle capillarity (due to deletion of VEGF in myocytes) are shown to reduce exercise performance (Olfert et al. 2009). The strong association between exercise capacity and muscle capillarity is also supported in selectively bred rats with high and low aerobic capacity (Howlett et al. 2003). Thus, the present findings are consistent with the evidence that skeletal muscle capillarity and muscle $\mathrm{O}_{2}$ conductance are critical determinants of aerobic exercise performance (Howlett et al. 2003). It is interesting to note, however, that $\dot{V}_{\mathrm{O}_{2} \text { max }}$ is not statistically different between MM mice and any of the other high aerobic capacity mouse lines, except during exercise in hypoxia (Rezende et al. 2006a,b). If $\mathrm{O}_{2}$ transport in the mouse is principally limited at the muscle-capillary interface, as implied by these and the other aforementioned data, then the greater capillarity in GA muscle of MM mice could help explain the advantage in $\dot{V}_{\mathrm{O}_{2} \max }$ seen during hypoxic exercise over Non-MM mice (Rezende et al. 2006a). Although these data do not establish a cause-and-effect relationship, they are in harmony with the view that VEGF-mediated increases in skeletal muscle capillarity contribute to the greater exercise capabilities observed in mice with an innate high aerobic capacity. Given the positive relationship noted for VEGF and C:F (Fig. 4) and the notable absence of an inverse relationship for TSP-1 and C:F (Fig. 4), it could be argued that VEGF may be the most critical determinant for muscle capillarity. More importantly, the fact that VEGF and TSP1 had different responses within the triceps surae muscles between these two high aerobic capacity lines (i.e. MM
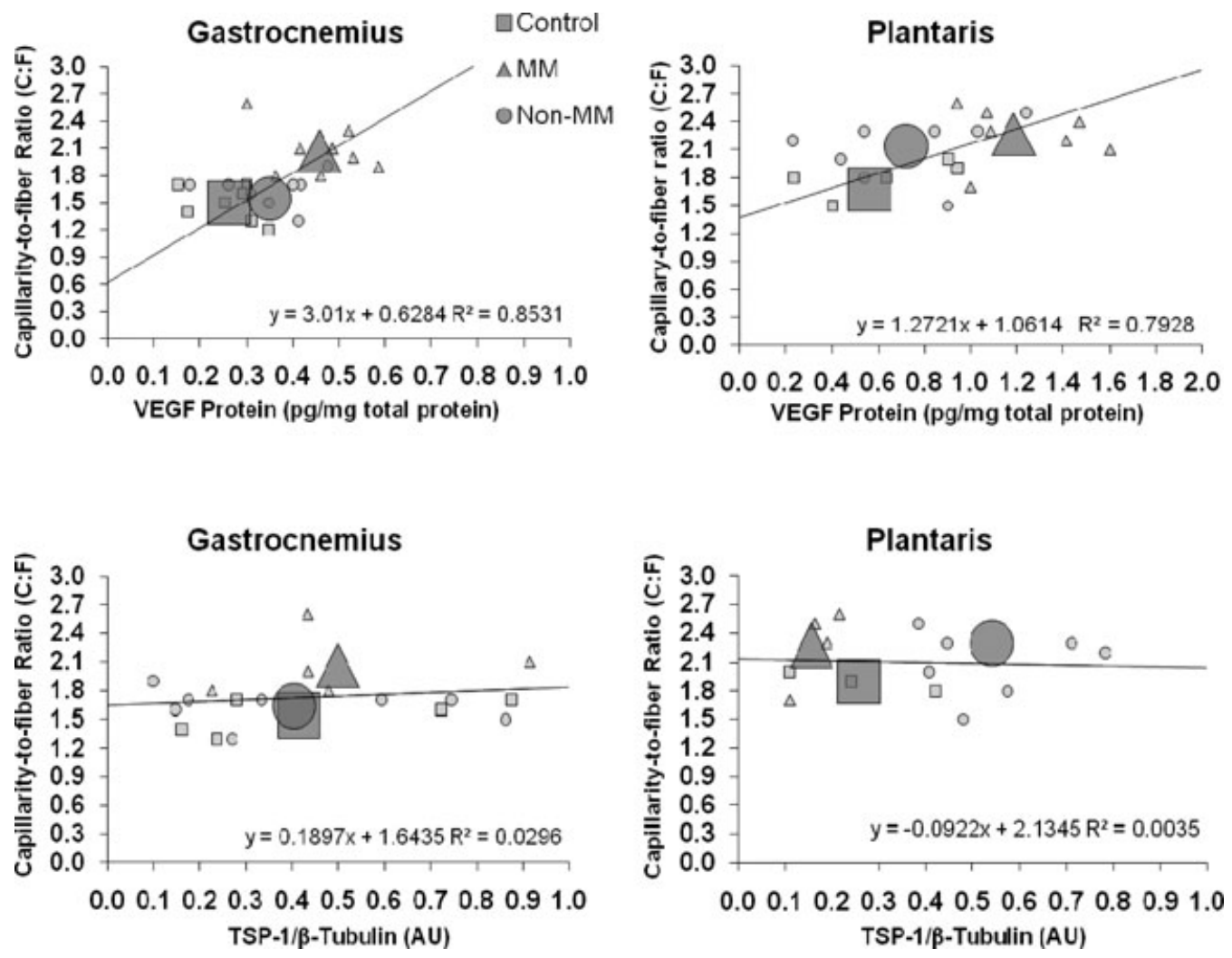

Figure 4. Correlation of muscle VEGF and TSP-1 levels to capillary-to-fibre ratio (C:F) Least-squares linear regression of C:F on basal VEGF and TSP-1 levels in the GA and PLT muscles of the three mouse lines. Large symbols indicate mean values for each line and small symbols are values for individual animals. Squares indicate control mice, triangles MM mice and circles Non-MM mice. 
versus Non-MM) suggests that basal angiogenic protein expression may be, at least in part, an inherited trait that may also account for some of the biological variability in exercise capacity.

\section{Genetic background may influence angiogenic responses to acute exercise}

In the context of exercise training, it has been shown in standard laboratory mice and rats that the VEGF response to acute exercise is attenuated in skeletal muscle post-training (Olfert et al. 2001; Malek \& Olfert, 2009). It has been postulated that this is a direct response to training-induced angio-adaptation (i.e. increased muscle capillarity, improved blood flow distribution to working muscle, and increased $\mathrm{O}_{2}$ conductance into the myocyte) that functions to relieve metabolic stress on the myocyte that is imposed by exercise. Given that local tissue hypoxia is thought to be among the key stimuli for the VEGF response to exercise (Levy et al. 1995; Breen et al. 1996; Richardson et al. 1999; Olfert et al. 2001), we have previously speculated that the attenuated VEGF response to an acute bout of exercise post-training is the result of increased muscle capillarity and greater $\mathrm{O}_{2}$ conductance during exercise (Olfert et al. 2001; Malek \& Olfert, 2009). The present data could be interpreted to suggest that a mechanism other than local hypoxia may be involved in the modulation of VEGF during acute responses to exercise. Indeed, if increased capillarity reduces local tissue hypoxia, and this is responsible for modulating the VEGF response, then it would be expected that an attenuated VEGF response to exercise would be seen in MM mice (which inherently displayed a greater number of capillaries in their muscle compared with normal mice). However, as it has also previously been shown that MM mice have increased aerobic metabolic enzyme activities in hindlimb muscles (Guderley et al. 2008), it could also be that MM mice simply have a much higher oxygen flux and thus the level of $\mathrm{O}_{2}$ within the muscles is relatively unchanged compared with Non-MM and control mice (despite the greater number of muscle capillaries). We also cannot exclude the possibility that other mechanisms, such as shear stress, local drops in muscle $\mathrm{pH}$, modulation of other matrix regulators and/or other angiogenic factors, may have indirect influences on the VEGF response to exercise.

Some support for modulation of other angiogenic factors comes from the TSP-1 data in the present study. Thrombospondin-1 is a potent inhibitor of angiogenesis and of VEGF directly (Gupta et al. 1999; Greenaway et al. 2007). In the MM mice, we found that TSP-1 levels were lower in the PLT in basal conditions and in the GA in the acute exercise conditions (Fig. 3). In both of these circumstances, VEGF is also increased. This supports the notion of an altered balance between positive (e.g. VEGF) and negative (e.g. TSP-1) angiogenic factors (Hanahan \&
Folkman, 1996; Olfert \& Birot, 2011). This altered balance would be another elegant way that the MM mice are genetically more able to adapt to an exercise stimulus.

Another potential explanation for the greater VEGF response to exercise in $\mathrm{MM}$ mice may be that the VEGF response to exercise is also genetically influenced. Although it is difficult to decipher the relative contribution of genetic versus environmental influences in setting an individual's exercise capacity, evidence supporting the genetic influences can be seen from a recent report involving fine genotype mapping of MM mice (Hartmann et al. 2008). Hartmann et al. (2008) localized the gene responsible for the mini-muscle phenotype to a $2.6335 \mathrm{Mb}$ of MMU11, a region that harbours $\sim 100$ expressed or predicted genes, many with known roles in muscle development and/or function. Upon examination of the genes within this region, we have identified two genes, Tnfsf12/TWEAK and Ephrin ligands/receptors, both of which have been shown to have some regulatory role in angiogenesis and/or endothelial cell proliferation (Helbling et al. 2000; Cheng et al. 2002; Donohue et al. 2003). What role, if any, these factors may exert on skeletal muscle VEGF remains to be determined, but it is tempting to speculate that these (or other) genes may support and/or enhance angiogenesis in MM mice.

It should be noted that our data are not the first to suggest that the VEGF response to exercise may be influenced by genetic background. Prior et al. (2006) have shown that genetic variants in the promoter region of the VEGF gene correlate with whole-body $\dot{V}_{\mathrm{O}_{2} \max }$ in humans. Similar results were also reported in response to hypoxia-mediated increased in VEGF gene expression in cultured myotubes (Prior et al. 2006). Our data, combined with these previous studies, imply that individual gene responses may influence exercise performance, and suggest that the VEGF response to exercise may, at least in part, be an inherited trait.

\section{Comparison of muscle mass and fibre-type profile among mouse lines}

It has previously been shown that MM mice have a significantly decreased triceps surae muscle mass (Syme et al. 2005; Guderley et al. 2006, 2008; McGillivray et al. 2009). Likewise, the present study shows that GA and PLT muscle mass was decreased in the MM mice when compared with the control and Non-MM mice (Table 1). Opposite to what is seen in the GA and PLT muscles, the SOL of the MM mice was increased over $130 \%$ (on a body mass-specific basis) when compared with both the control mice and the Non-MM line (Table 1).

In mice, the GA has an aerobic profile that is mixed, with superficial portions of the muscle containing mainly type IIb/x fibres, whereas the deep portions contains many type IIa and type I fibres (Guderley et al. 2008; Wong 
et al. 2009). In contrast, the SOL is comprised primarily of type I or IIa fibres. Assessment of fibre type in these mice reveals that MM mice have a greater proportion of type I than type II myofibres compared with control and Non-MM mice (Table 2). These findings are supported by earlier observations that MM mice have increased type I and type IIa fibres along with decreased type IIb fibres in the medial GA muscle (Guderley et al. 2008). This myofibre composition is consistent with the elevated C:F ratio found in the GA of the MM mice in the present study, further supporting the notion of a greater oxidative muscle metabolic profile capable of supporting greater aerobic capacity.

This also raises the issue of the influence of muscle mass and/or fibre type/size on skeletal muscle aerobic performance. It could be argued that greater cardiac output (as indicated by larger cardiac mass in both absolute terms and relative to body size) could itself contribute to improved exercise capacity in MM mice (Table 1). Perhaps the strongest argument against this hypothesis is provided by the Non-MM mice, which exhibit an equally high aerobic endurance capacity to the MM mice but do not have greater cardiac mass (in absolute or relative terms) compared with control animals (Garland et al. 2002; Table 1). Moreover, we would also contend (irrespective of any improvements in central cardiac function) that blood flow to the working muscle is also controlled by local metabolic demand. In combination with the greater muscle metabolic potential of MM mice, such as increased mitochondrial enzymatic activity (Guderley et al. 2006), it is perhaps debatable whether greater cardiac function in these MM mice might be considered as improved or simply an attempt to keep up with metabolic demand of the working muscle. Nonetheless, there are presently no data which document cardiac output or function in these mice, thus we do not know if cardiac function is altered.

In summary, these data generally support the notion that skeletal muscle angiogenesis in physiological conditions is likely to be controlled by a balance between pro- and anti-angiogenic factors, and also suggest that the expression of VEGF versus TSP-1 may differ among muscles. Moreover, the observation that basal VEGF and TSP-1 expression, and the VEGF and TSP-1 response to exercise, were different between the two high aerobic capacity mouse lines seems to suggest first, that a mechanism other than local hypoxia might be regulating the VEGF response to exercise and second, that the VEGF response to exercise may, in part, be genetically determined.

\section{References}

Andersen P \& Henriksson J (1977). Capillary supply of the quadriceps femoris muscle of man: adaptive response to exercise. J Physiol 270, 677-690.
Birot OJ, Koulmann N, Peinnequin A \& Bigard XA (2003). Exercise-induced expression of vascular endothelial growth factor mRNA in rat skeletal muscle is dependent on fibre type. J Physiol 552, 213-221.

Breen EC, Johnson EC, Wagner H, Tseng H-M, Sung LA \& Wagner PD (1996). Angiogenic growth factor mRNA responses in muscle to a single bout of exercise. J Appl Physiol 81, 355-361.

Cheng N, Brantley DM \& Chen J (2002). The ephrins and Eph receptors in angiogenesis. Cytokine Growth Factor Rev 13, 75-85.

Donohue PJ, Richards CM, Brown SA, Hanscom HN, Buschman J, Thangada S, Hla T, Williams MS \& Winkles JA (2003). TWEAK is an endothelial cell growth and chemotactic factor that also potentiates FGF-2 and VEGF-A mitogenic activity. Arterioscler Thromb Vasc Biol 23, 594-600.

Feder ME, Garland T Jr, Marden JH \& Zera AJ (2010). Locomotion in response to shifting climate zones: not so fast. Annu Rev Physiol 72, 167-190.

Garland T Jr, Kelly SA, Malisch JL, Kolb EM, Hannon RM, Keeney BK, Van Cleave SL \& Middleton KM (2011). How to run far: multiple solutions and sex-specific responses to selective breeding for high voluntary activity levels. Proc Biol Sci 278, 574-581.

Garland T Jr, Morgan MT, Swallow JG, Rhodes JS, Girard I, Belter JG \& Carter PA (2002). Evolution of a small-muscle polymorphism in lines of house mice selected for high activity levels. Evolution 56, 1267-1275.

Gavin TP \& Wagner PD (2001). Effect of short-term exercise training on angiogenic growth factor gene responses in rats. J Appl Physiol 90, 1219-1226.

Greenaway J, Lawler J, Moorehead R, Bornstein P, Lamarre J \& Petrik J (2007). Thrombospondin-1 inhibits VEGF levels in the ovary directly by binding and internalization via the low density lipoprotein receptor-related protein-1 (LRP-1). J Cell Physiol 210, 807-818.

Guderley H, Houle-Leroy P, Diffee GM, Camp DM \& Garland T Jr (2006). Morphometry, ultrastructure, myosin isoforms, and metabolic capacities of the "mini muscles" favoured by selection for high activity in house mice. Comp Biochem Physiol B Biochem Mol Biol 144, 271-282.

Guderley H, Joanisse DR, Mokas S, Bilodeau GM \& Garland T $\mathrm{Jr}$ (2008). Altered fibre types in gastrocnemius muscle of high wheel-running selected mice with mini-muscle phenotypes. Comp Biochem Physiol B Biochem Mol Biol 149, 490-500.

Gupta K, Gupta P, Wild R, Ramakrishnan S \& Hebbel RP (1999). Binding and displacement of vascular endothelial growth factor (VEGF) by thrombospondin: effect on human microvascular endothelial cell proliferation and angiogenesis. Angiogenesis 3, 147-158.

Gustafsson T, Knutsson A, Puntschart A, Kaijser L, Nordqvist AC, Sundberg CJ \& Jansson E (2002). Increased expression of vascular endothelial growth factor in human skeletal muscle in response to short-term one-legged exercise training. Pflugers Arch 444, 752-759.

Hanahan D \& Folkman J (1996). Patterns and emerging mechanisms of the angiogenic switch during tumorigenesis. Cell 86, 353-364. 
Hartmann J, Garland T Jr, Hannon RM, Kelly SA, Muñoz G \& Pomp D (2008). Fine mapping of "mini-muscle," a recessive mutation causing reduced hindlimb muscle mass in mice. J Hered 99, 679-687.

Helbling PM, Saulnier DM \& Brandli AW (2000). The receptor tyrosine kinase EphB4 and ephrin-B ligands restrict angiogenic growth of embryonic veins in Xenopus laevis. Development 127, 269-278.

Henderson KK, Wagner H, Favret F, Britton SL, Koch LG, Wagner PD \& Gonzalez NC (2002). Determinants of maximal $\mathrm{O}_{2}$ uptake in rats selectively bred for endurance running capacity. J Appl Physiol 93, 1265-1274.

Houle-Leroy P, Guderley H, Swallow JG \& Garland T Jr (2003). Artificial selection for high activity favors mighty mini-muscles in house mice. Am J Physiol Regul Integr Comp Physiol 284, R433-R443.

Howlett RA, Gonzalez NC, Wagner HE, Fu Z, Britton SL, Koch LG \& Wagner PD (2003). Genetic models in applied physiology: skeletal muscle capillarity and enzyme activity in rats selectively bred for running endurance. J Appl Physiol 94, 1682-1688.

Howlett RA, Kirkton SD, Gonzalez NC, Wagner HE, Britton SL, Koch LG \& Wagner PD (2009). Peripheral oxygen transport and utilization in rats following continued selective breeding for endurance running capacity. J Appl Physiol 106, 1819-1825.

Koch LG \& Britton SL (2001). Artificial selection for intrinsic aerobic endurance running capacity in rats. Physiol Genomics 5, 45-52.

Koch LG, Britton SL, Barbato JC, Rodenbaugh DW \& DiCarlo SE (1999). Phenotypic differences in cardiovascular regulation in inbred rat models of aerobic capacity. Physiol Genomics 1, 63-69.

Koteja P, Garland T Jr, Sax JK, Swallow JG \& Carter PA (1999). Behaviour of house mice artificially selected for high levels of voluntary wheel running. Anim Behav 58, 1307-1318.

Lee S, Chen TT, Barber CL, Jordan MC, Murdock J, Desai S, Ferrara N, Nagy A, Roos KP \& Iruela-Arispe ML (2007). Autocrine VEGF signaling is required for vascular homeostasis. Cell 130, 691-703.

Levy AP, Levy NS, Wegner S \& Goldberg MA (1995). Transcriptional regulation of rat vascular endothelial growth factor gene by hypoxia. J Biol Chem 270, 13333-13340.

Lloyd PG, Prior BM, Yang HT \& Terjung RL (2003). Angiogenic growth factor expression in rat skeletal muscle in response to exercise training. Am J Physiol Heart Circ Physiol 284, H1668-H1678.

McGillivray DG, Garland T Jr, Dlugosz EM, Chappell MA \& Syme DA (2009). Changes in efficiency and myosin expression in the small-muscle phenotype of mice selectively bred for high voluntary running activity. J Exp Biol 212, 977-985.

Malek MH \& Olfert IM (2009). Global deletion of thrombospondin-1 increases cardiac and skeletal muscle capillarity and exercise capacity. Exp Physiol 94, 749-760.

Meek TH, Lonquich BP, Hannon RM \& Garland T Jr (2009). Endurance capacity of mice selectively bred for high voluntary wheel running. J Exp Biol 212, 2908-2917.
Olfert IM \& Birot O (2011). Importance of anti-angiogenic factors in the regulation of skeletal muscle angiogenesis. Microcirculation 18, 316-330.

Olfert IM, Breen EC, Gavin TP \& Wagner PD (2006). Temporal thrombospondin-1 mRNA response in skeletal muscle exposed to acute and chronic exercise. Growth Factors 24, 253-259.

Olfert IM, Breen EC, Mathieu-Costello O \& Wagner PD (2001). Skeletal muscle capillarity and angiogenic mRNA levels after exercise training in normoxia and chronic hypoxia. J Appl Physiol 91, 1176-1184.

Olfert IM, Howlett RA, Tang K, Dalton ND, Gu Y, Peterson KL, Wagner PD \& Breen EC (2009). Muscle-specific VEGF deficiency greatly reduces exercise endurance in mice. J Physiol 578, 1755-1767.

Olfert IM, Howlett RA, Wagner PD \& Breen EC (2010). Myocyte vascular endothelial growth factor is required for exercise-induced skeletal muscle angiogenesis. Am J Physiol Regul Integr Comp Physiol 299, R1059-R1067.

Prior SJ, Hagberg JM, Paton CM, Douglass LW, Brown MD, McLenithan JC \& Roth SM (2006). DNA sequence variation in the promoter region of the VEGF gene impacts VEGF gene expression and maximal oxygen consumption. Am J Physiol Heart Circ Physiol 290, H1848-H1855.

Rezende EL, Garland T Jr, Chappell MA, Malisch JL \& Gomes FR (2006a). Maximum aerobic performance in lines of Mus selected for high wheel-running activity: effects of selection, oxygen availability and the mini-muscle phenotype. J Exp Biol 209, 115-127.

Rezende EL, Gomes FR, Malisch JL, Chappell MA \& Garland T $\mathrm{Jr}(2006 b)$. Maximal oxygen consumption in relation to subordinate traits in lines of house mice selectively bred for high voluntary wheel running. J Appl Physiol 101, 477-485.

Richardson RS, Wagner H, Mudaliar SR, Henry R, Noyszewski EA \& Wagner PD (1999). Human VEGF gene expression in skeletal muscle: effect of acute normoxic and hypoxic exercise. Am J Physiol Heart Circ Physiol 277, H2247-H2252.

Rosenblatt JD, Kuzon WM, Plyley MJ, Pynn BR \& McKee NH (1987). A histochemical method for the simultaneous demonstration of capillaries and fiber type in skeletal muscle. Stain Technol 62, 85-92.

Swallow JG, Carter PA \& Garland T Jr (1998a). Artificial selection for increased wheel-running behavior in house mice. Behav Genet 28, 227-237.

Swallow JG, Garland T Jr, Carter PA, Zhan WZ \& Sieck GC $(1998 b)$. Effects of voluntary activity and genetic selection on aerobic capacity in house mice (Mus domesticus). J Appl Physiol 84, 69-76.

Swallow JG, Hayes JP, Koteja P \& Garland T (2009). Selection experiments and experimental evolution of performance and physiology. In Experimental Evolution: Concepts, Methods, and Application of Selection Experiments, ed. Garland TJ \& Rose MR, pp. 301-351. University of California Press, Berkeley, CA, USA.

Swallow JG, Koteja P, Carter PA \& Garland T (1999). Artificial selection for increased wheel-running activity in house mice results in decreased body mass at maturity. J Exp Biol 202, 2513-2520. 
Swallow JG, Koteja P, Carter PA \& Garland T Jr (2001). Food consumption and body composition in mice selected for high wheel-running activity. J Comp Physiol B 171, 651-659.

Syme DA, Evashuk K, Grintuch B, Rezende EL \& Garland T Jr (2005). Contractile abilities of normal and "mini" triceps surae muscles from mice (Mus domesticus) selectively bred for high voluntary wheel running. J Appl Physiol 99, 1308-1316.

Tang K, Breen EC, Gerber HP, Ferrara NM \& Wagner PD (2004). Capillary regression in vascular endothelial growth factor-deficient skeletal muscle. Physiol Genomics 18, 63-69.

Wagner PD (2003). Heterogeneity of skeletal muscle perfusion and metabolism. J Appl Physiol 95, 22022203.

Waters RE, Rotevatn S, Li P, Annex BH \& Yan Z (2004). Voluntary running induces fiber type-specific angiogenesis in mouse skeletal muscle. Am J Physiol Cell Physiol 287, C1342-C1348.
Wisloff U, Najjar SM, Ellingsen O, Haram PM, Swoap S, Al-Share Q, Fernstrom M, Rezaei K, Lee SJ, Koch LG \& Britton SL (2005). Cardiovascular risk factors emerge after artificial selection for low aerobic capacity. Science 307, 418-420.

Wong LE, Garland T Jr, Rowan SL \& Hepple RT (2009). Anatomic capillarization is elevated in the medial gastrocnemius muscle of mighty mini mice. J Appl Physiol 106, 1660-1667.

\section{Acknowledgements}

The authors wish to thank Harrieth Wagner and Darryl Neusome for their assistance in the laboratory in processing muscle tissue samples, and we thank Daniel Fulks for critically reading the manuscript. This project was supported by funding from West Virginia University School of Medicine (I.M.O.), TobaccoRelated Disease Research Program Grant 14KT-0091 (I.M.O.), American Heart Association Grant 10BGIA3630002 (I.M.O.) and National Science Foundation (IOB-0543429). 